Stefan M. Golder

\title{
Migration und Arbeitsmarkt
}




\section{Stefan M. Golder}

\section{Migration und Arbeitsmarkt}

Die Entwicklung der schweizerischen Volkswirtschaft ist stark von der Zuwanderung ausländischer Arbeitskräfte geprägt. Strukturelle Veränderungen im Migrationsangebot und in der Migrationsnachfrage haben dazu geführt, daß die schweizerische Migrationspolitik vor großen Herausforderungen steht. Die Analyse der Arbeitsmarktperformance, d.h. der Vergleich der Beschäftigungsund Einkommenssituation von In- und Ausländern, ermöglicht eine umfassende Evaluation dieses Zusammenhangs. Die Analyse der Arbeitsmarktperformance erfolgtüber lineare sowie Probit Regressionen im Rahmen einer Querschnittsanalyse. Basierend auf den Erkenntnissen dieser Überlegungen werden Vorschläge zur Neuorientierung der schweizerischen Migrationspolitik abgeleitet.

Stefan M. Golder, Jahrgang 1970, absolvierte sein Studium der Wirtschaftswissenschaften mit Schwerpunkt Volkswirtschaft an der Universität Basel. 1995/96 nahm er am Nachdiplomstudiengang „Advanced Studies in International Economic Policy Research" des Kieler Instituts für Weltwirtschaft teil. Forschungsaufenthalt am Institut für Wirtschaftspolitik der Universität der Bundeswehr Hamburg. Promotion 1998. Stefan M. Golder ist gegenwärtig als wissenschaftlicher Beamter in der Sektion IWF und internationale Finanzierungsfragen der Eidgenössischen Finanzverwaltung in Bern tätig. 
Migration und Arbeitsmarkt 


\section{SCHRIFTEN ZUR WIRTSCHAFTSTHEORIE UND WIRTSCHAFTSPOLITIK}

Herausgegeben von

Rolf Hasse, Wolf Schäfer, Thomas Straubhaar und Klaus W. Zimmermann

\section{Band II}

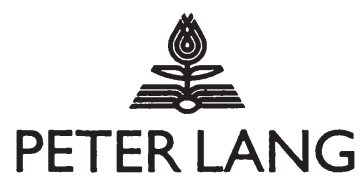

Frankfurt am Main - Berlin - Bern - New York - Paris - Wien 


\section{Stefan M. Golder}

\section{Migration und Arbeitsmarkt}

Eine empirische Analyse der Performance von Ausländern in der Schweiz

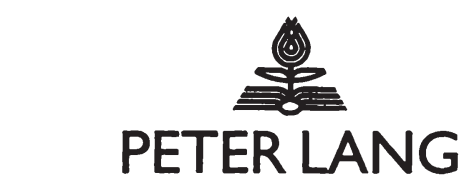

Europäischer Verlag der Wissenschaften 
Die Deutsche Bibliothek - CIP-Einheitsaufnahme

Golder, Stefan M.:

Migration und Arbeitsmarkt : eine empirische Analyse der Performance von Ausländern in der Schweiz / Stefan M.

Golder. - Frankfurt am Main ; Berlin ; Bern ; New York ; Paris ;

Wien : Lang, 1999

(Schriften zur Wirtschaftstheorie und Wirtschaftspolitik ;

Bd. 11)

Zugl.: Basel, Univ., Diss., 1998

ISBN 3-631-33769-8

Open Access: The online version of this publication is published on www.peterlang.com and www.econstor.eu under the international Creative Commons License CC-BY 4.0. Learn more on how you can use and share this work: http://creativecommons. org/licenses/by/4.0.

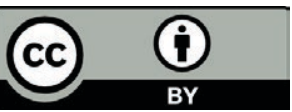

This book is available Open Access thanks to the kind support of ZBW - Leibniz-Informationszentrum Wirtschaft.

Gedruckt auf alterungsbeständigem, säurefreiem Papier.

\author{
ISSN 1433-1519 \\ ISBN 3-631-33769-8 \\ ISBN 978-3-631-74998-2 (eBook) \\ (C) Peter Lang GmbH \\ Europäischer Verlag der Wissenschaften \\ Frankfurt am Main 1999 \\ Alle Rechte vorbehalten.
}

Das Werk einschließlich aller seiner Teile ist urheberrechtlich geschützt. Jede Verwertung außerhalb der engen Grenzen des Urheberrechtsgesetzes ist ohne Zustimmung des Verlages unzulässig und strafbar. Das gilt insbesondere für Vervielfältigungen, Übersetzungen, Mikroverfilmungen und die Einspeicherung und Verarbeitung in elektronischen Systemen.

Printed in Germany 124567 


\section{VORWORT}

Diese Studie entstand während meiner Zeit als Forschungsassistent an der Universität Basel sowie eines Forschungsaufenthaltes am Institut für Wirtschaftspolitik der Universität der Bundeswehr in Hamburg. Sie wurde als Dissertation an der Wirtschaftswissenschaftlichen Fakultät der Universität Basel angenommen.

Ich danke meinem Doktorvater Prof. Dr. Silvio Borner nicht nur für die kompetente fachliche Betreuung und die gute Zusammenarbeit, die massgeblich zum Gelingen dieser Dissertation beigetragen haben, sondern auch für sein Vertrauen und den Freiraum den er mir während meines Forschungsaufenthaltes gewährte. Mein Dank gilt aber auch meinem Korreferenten Prof. Dr. Thomas Straubhaar, dem Direktor des Instituts für Wirtschaftspolitik an der Universität der Bundeswehr Hamburg, der mir die Chance gegeben hat, meine Forschungsarbeiten an seinem Institut voranzutreiben. An dieser Stelle möchte ich auch meinen Kollegen Achim, Hubertus, Peter und Stefan in Hamburg für Ihre vielen Kommentare und Anregungen danken.

Hervorheben möchte ich desweiteren George Sheldon, der mir in der Schlussphase dieser Arbeit in vielen Diskussionen wichtige Hinweise zur inhaltlichen Verbesserung dieser Arbeit geben konnte. Mein Dank geht auch an Michel Kolly vom Bundesamt für Statistik, der mir immer wieder kompetent und äusserst hilfsbereit Fragen zu den Daten der Schweizerischen Arbeitskräfteerhebung beantworten konnte. Diese Arbeit wäre nicht ohne die finanzielle Unterstützung des WWZ-Fördervereins, des Schweizerischen Nationalfonds sowie der Max Geldner Stiftung zustandegekommen. Diesen Institutionen sei an dieser Stelle herzlich gedankt.

Schliesslich möchte ich meinen Eltern sehr dafür danken, dass sie mich während der vergangenen Jahre immer unterstützt und motiviert haben.

Mein grösster Dank geht an Friederike, für ihren speziellen Beitrag zum Gelingen dieser Arbeit.

Basel, im September 1998 
Stefan Golder - 978-3-631-74998-2

Downloaded from PubFactory at 01/11/2019 09:25:33AM

via free access 


\section{INHALTSVERZEICHNIS}

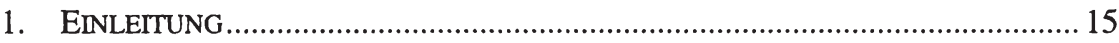

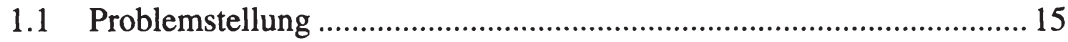

1.1.1 Wachsendes Migrationspotential ........................................... 15

1.1.2 Handlungsbedarf für die Schweiz ............................................. 16

1.2 Begriffsdefinitionen und Aufbau der Studie ........................................ 17

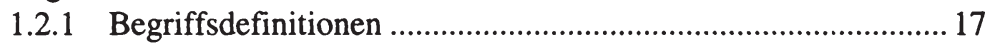

1.2.2 Aufbau der Studie und wichtigste Ergebnisse.......................... 19

1.3 Eingrenzung des Untersuchungsgegenstandes..................................21

TEIL 1 (ÜBERBLICK): INTERNATIONALE ZUWANDERUNG UND DER ARBEITSMARKT: EINIGE GRUNDLEGENDE FAKTEN UND ZUSAMMENHÄNGE

2. MigRATIONSERFAHRUNGEN DER SCHWEIZ - HISTORISCHER ÜBERBLICK VOR DEM HINTERGRUND DER ENTWICKLUNG AUF DEM ARBEITSMARKT 23

2.1 Liberale Zulassungspolitik .................................................................. 24

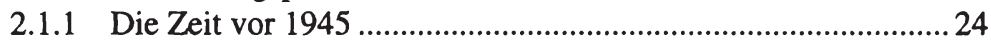

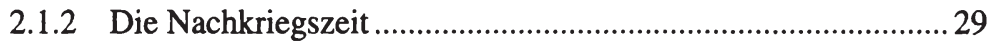

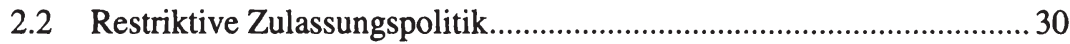

2.2.1 Betriebsweise Plafonierung (1963 - 1970) ............................... 30

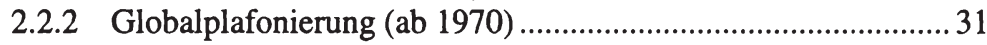

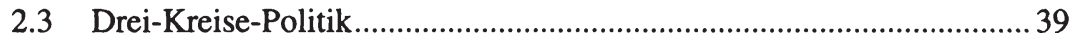

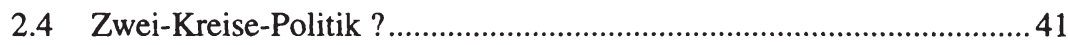

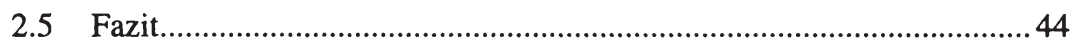

3. DESKRIPTIVE ANALYSE DER ARBEITSMARKTPERFORMANCE ...........................45

3.1 Ausländeranteil in der Schweiz und im internationalen Vergleich....... 45

3.2 Demographische Merkmale der ausländischen Wohnbevölkerung ...... 46

3.3 Beschäftigungsperformance von Einwanderern...................................50

3.4 Einkommensperformance von Einwanderern .......................................5

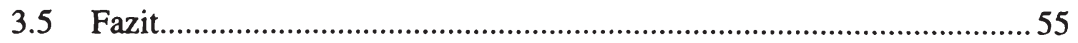


4. ÖKONOMISCHE ANSÄTZE ZUR ERKLÄRUNG VON MIGRATION .........................56

4.1 Der neoklassische Ansatz......................................................................5

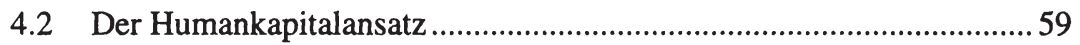

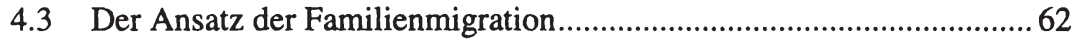

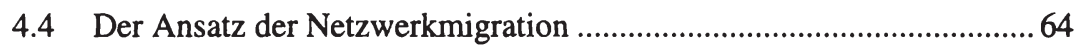

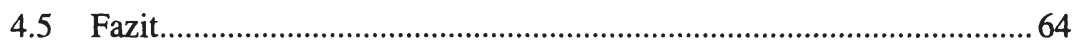

5. ARBEITSMARKTPERFORMANCE DER EINWANDERER .....................................66

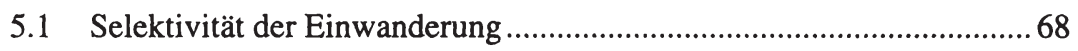

5.1.1 Bestimmungsfaktoren für die Selektivität der

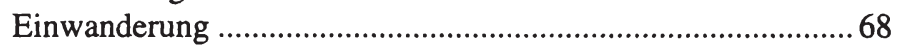

5.1.2 Bedeutung von Unterschieden zwischen Herkunfts- und

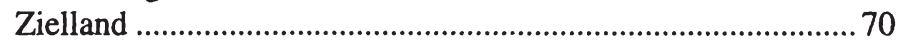

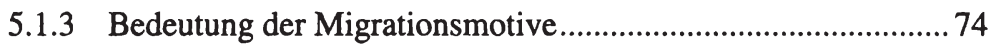

5.2 Internationale Transferierbarkeit von Humankapital ........................... 74

5.2.1 Bestimmungsfaktoren für die Transferierbarkeit von

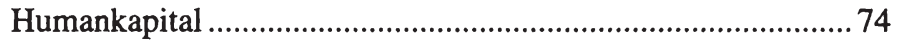

5.2.2 Bedeutung der Ähnlichkeiten zwischen Herkunfts- und

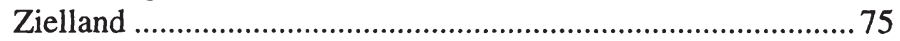

5.2.3 Bedeutung der Migrationsmotive............................................. 76

5.3 Arbeitsmarktassimilation der Einwanderer......................................... 78

5.3.1 Bestimmungsfaktoren für die Assimilation der

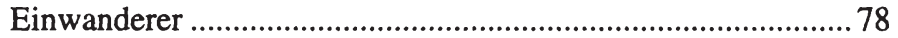

5.3.2 Bedeutung der Aufenthaltsdauer im Zielland ..........................79

5.3.3 Bedeutung des Einwanderungszeitpunktes ............................... 82

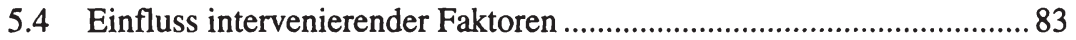

5.4.1 Nachfrage nach ausländischen Arbeitskräften ......................... 84

5.4.2 Rechtliche Rahmenbedingungen der Migrationspolitik............87

5.4.3 Faktormobilität und Güterhandel ................................................ 88

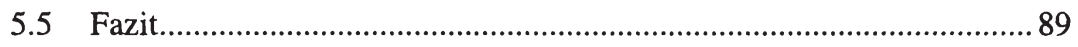

6. DISKRIMINIERUNG VON EINWANDERERN AUF DEM ARBEITSMARKT .................91

6.1 Bedeutung und Definition von Diskriminierung .................................. 92

6.2 Ansatzpunkte für Diskriminierung bei der Beschäftigung von

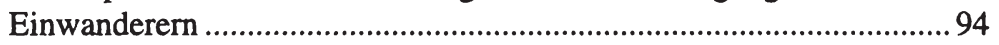

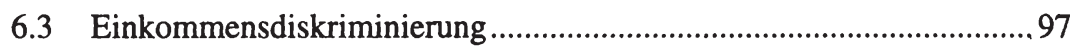

6.3.1 Persönliche Vorurteile............................................................97

6.3.1.1 Vorurteile von Arbeitgebern ...................................... 97 
6.3.1.2 Vorurteile der Arbeitnehmer ...................................... 100

6.3.1.3 Vorurteile der Kunden ........................................... 100

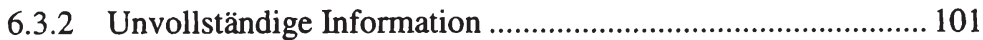

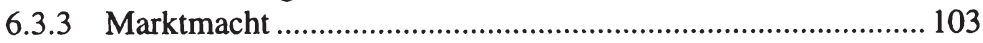

6.3.3.1 Monopsonistische Firmen auf dem Arbeitsmarkt..... 104

6.3.3.2 Gewerkschaften als Monopolisten ............................ 105

6.3.3.3 Regierung als Monopolist ........................................ 105

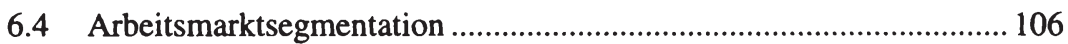

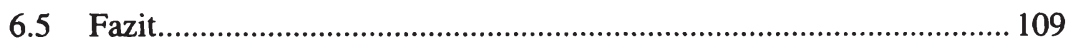

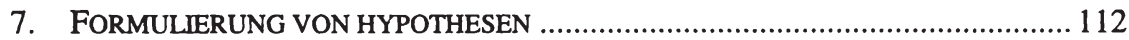

7.1 Folgerungen aus der deskriptiven und der theoretischen Analyse ...... 113

7.2 Hypothesen zur Arbeitsmarktperformance ........................................ 114

7.2.1 Allgemeine Hypothesen .......................................................... 114

7.2.2 Spezifische Hypothesen zur Beschäftigungsperformance....... 115

7.2.3 Spezifische Hypothesen zur Einkommensperformance .......... 116

TELL 3 (EMPIRIE): EMPIRISCHE ANALYSE DER

ARBEITSMARKTPERFORMANCE..................................................... 118

8. UNTERSUCHUNGSMETHODE UND EMPIRISCHE DATENBASIS ......................... 118

8.1 Nationalitäten- und einwanderungsspezifische Merkmale ................. 119

8.1.1 Abgrenzung von Nationalitätengruppen .................................119

8.1.2 Abgrenzung von Einwanderungskohorten ............................. 120

8.2 Assimilierungs-, Kohorten-, Alters- und Periodeneffekte .................. 122

8.3 Interaktion zwischen Nationalität und Einwanderungskohorte .......... 126

8.4 Empirische Datenbasis: Die Schweizerische

Arbeitskräfteerhebung (SAKE)..................................................... 129

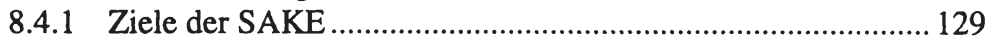

8.4.2 Definition von Erwerbstätigkeit und Erwerbslosigkeit ........... 130

8.4.3 Bedeutung der SAKE für die Wirtschafts- und Sozialstatistik ...................................................................... 133

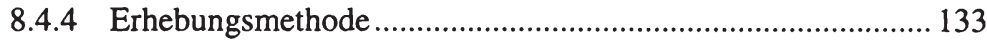

8.4.5 Aussagekraft und Grenzen der SAKE.................................. 135

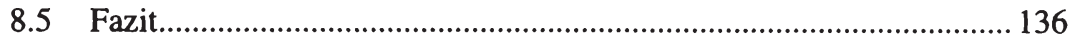

9. EMPIRISCHE ANALYSE DER BESCHÄFTIGUNGSPERFORMANCE ....................... 138

9.1 Deskriptive Analyse der Beschäftigungsperformance ....................... 138

9.1.1 Höchste Abgeschlossene Ausbildung ................................... 139

9.1.2 Berufliche Stellung........................................................... 142 


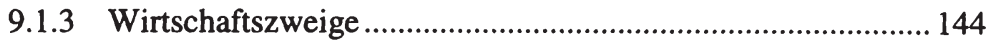

9.1.4 Erwerbsbeteiligung und Beschäftigungsgrad ......................... 147

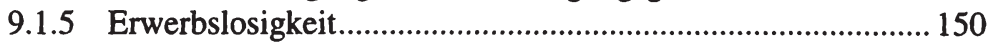

9.2 Empirische Analyse der Erwerbslosigkeit .......................................152

9.2.1 Methodische Vorgehensweise............................................. 153

9.2.1.1 Methodische Ansätze und Probleme: Probit- und Logit-Modelle ........................................................ 154

9.2.1.2 Modellspezifikation ...............................................157

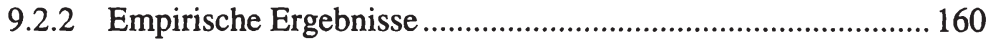

9.3 Empirische Analyse der Arbeitsmarksegmentation ............................ 164

9.3.1 Methodische Vorgehensweise............................................. 165

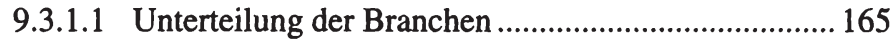

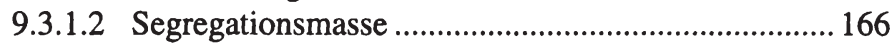

9.3.2 Empirische Ergebnisse ......................................................... 167

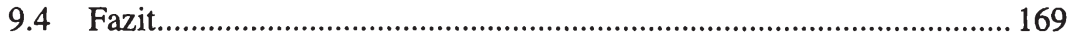

10. EMPIRISCHE ANALYSE DER EINKOMMENSPERFORMANCE ............................ 171

10.1 Deskriptive Analyse der Einkommensperformance............................ 172

10.1.1 Einkommenshöhe nach Nationalitätengruppen....................... 172

10.1.2 Einkommenshöhe nach Einwanderungskohorten .................. 174

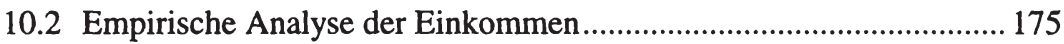

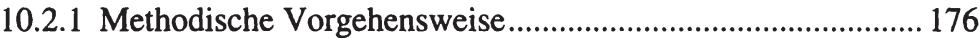

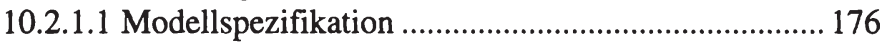

10.1.1.3 Selektivitäts-Bias Problematik................................. 180

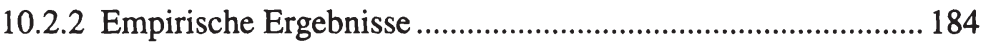

10.3 Empirische Analyse der Einkommensdiskriminierung....................... 191

10.3.1 Methodische Vorgehensweise ................................................ 191

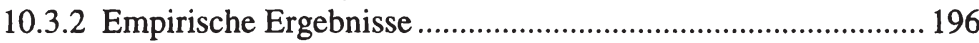

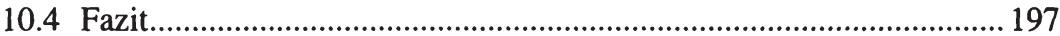

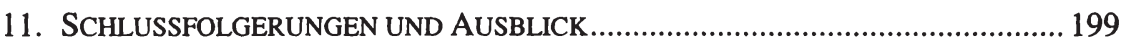

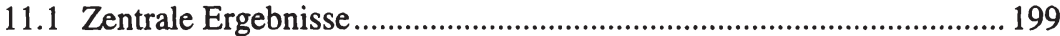

11.1.1 Ergebnisse der Theoretischen Analyse................................. 199

11.1.2 Ergebnisse der Empirischen Analyse ...................................200

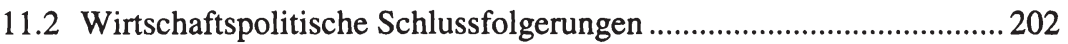

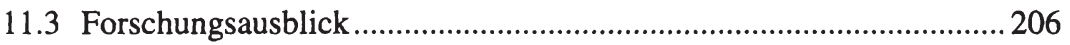

11.3.1 Vertiefungsmöglichkeiten der Analyse ..................................206

11.3.2 Erweiterungsmöglichkeiten der Analyse................................ 209

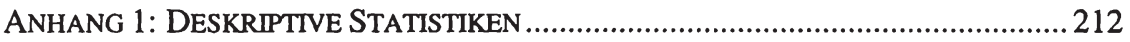

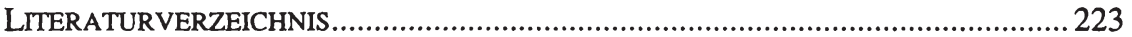




\section{ABBILDUNGSVERZEICHNIS}

Abb. 4.1: Ökonomische Ansätze zur Erklärung von Migration........................57

Abb. 5.1: Bestimmungsfaktoren der Arbeitsmarktperformance der Ein-

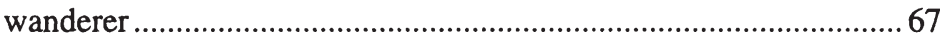

Abb. 6.1: Arbeitsmarktdiskriminierung induzierende Faktoren .....................91

Abb. 6.2: Lohndifferentiale aufgrund arbeitgeberseitiger Vorurteile............... 98

Abb. 6.3: Häufigkeitsverteilung der Arbeitsproduktivität von In- und Ausländern

Abb. 7.1: Vorgehensweise zur empirischen Analyse der Arbeitsmarktper-

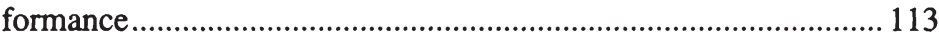

Abb. 8.1: Einkommensentwicklung dreier unterschiedlicher Einwanderungskohorten

\section{TABELLENVERZEICHNIS}

Tab. 2.1: Entwicklung des Ausländerbestandes in der Schweiz, 1850 -

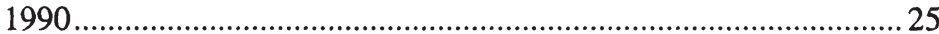

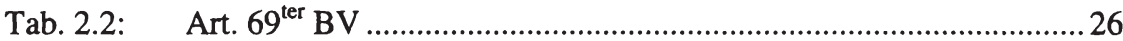

Tab. 2.3: Die Bewilligungskategorien des schweizerischen Ausländer-

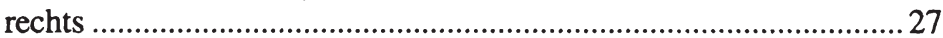

Tab. 2.4: Einreisen in die Schweiz nach Motiven, 1997 ................................ 28

Tab. 2.5: Ein- und Auswanderung erwerbstätiger Ausländer, 1970 -

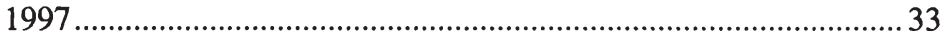

Tab. 2.6: Ziele der schweizerischen Ausländerpolitik.....................................36

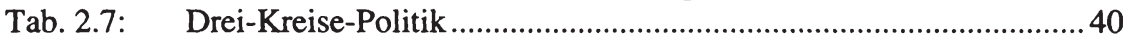

Tab. 2.8: Aufenthaltsbewilligungen gemäss Zwei-Kreise-Politik................... 43

Tab. 3.1: Ausländeranteil an der Bevölkerung und Beschäftigung in ausgewählten OECD Staaten, 1993 …....................................................46

Tab. 3.2: Zuwanderung in die Schweiz (in Prozent), 1960 - 1997 ................. 47

Tab. 3.3: Erwerbstätige ausländische Bevölkerung nach Staatsangehörigkeit, 1973-1997

Tab. 3.3: Erwerbstätige ausländische Bevölkerung nach Staatsangehörigkeit, 1973-1997 (Fortsetzung).

Tab. 3.4: Ausländische Erwerbstätige nach Aufenthaltskategorien, 1970

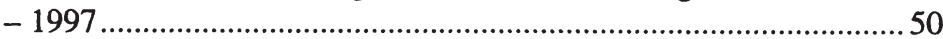

Tab. 3.5: Erwerbsquoten von Ausländern und Schweizern, 1960 - 1997.......51

Tab. 3.6: Arbeitslosenquoten von Schweizern und Ausländern, 1976 1997 
Tab. 3.7: Erwerbstätige Bevölkerung nach sozio-professionellen Kategorien, 1990 ......

Tab. 3.8: Erwerbstätige Bevölkerung nach Wirtschaftszweigen, 1970 1990 .

Tab. 3.9: Erwerbstätige nach jährlichem Bruttoerwerbseinkommen in CHF, 1995 54

Tab. 5.1: Zentrale Aussagen des Modells von BORJAS

Tab. 6.1: Ansatzpunkte zur Diskriminierung ausländischer Arbeitnehmer .... 95

Tab. 6.2: Theorie dualer Arbeitsmärkte. 108

Tab. 6.3: Folgen von Einkommensdiskriminierung und Arbeitsmarktsegmentierung für in- und ausländische Arbeitskräfte

Tab. 8.1: Unterscheidungsmerkmale zur Konstruktion von Nationalitätengruppen

Tab. 8.2: Definition verschiedener Nationalitätengruppen.

Tab. 8.3: Definition verschiedener Einwanderungskohorten

Tab. 8.4: Zentrale Ergebnisse der Analyse von CHISWICK. 123

Tab. 8.5: Determinanten der Einkommensunterschiede zwischen Einwanderern und Einheimischen

Tab. 8.6: Einwanderungskohorten nach Nationalitätengruppen (in Prozent).

Tab. 8.7: Nationalitätengruppen nach Einwanderungskohorten (in Prozent).....

Tab. 8.8: Zielsetzungen der Schweizerischen Arbeitskräfteerhebung (SAKE).

Tab. 8.9: Definition zentraler Begriffe zur Erwerbstätigkeit in der SAKE ... 130

Tab. 8.10: Statistische Quellen zur Erwerbstätigkeit und Beschäftigung:

Regionalisierungsgrad und erfasste Personengruppen

Tab. 9.1: Höchste abgeschlossene Ausbildung der erwerbstätigen Bevölkerung nach Nationalitätengruppen (in Prozent).

Tab. 9.2: Höchste abgeschlossene Ausbildung der erwerbstätigen Bevölkerung nach Einwanderungskohorten (in Prozent)...

Tab. 9.3: Berufliche Stellung der erwerbstätigen Bevölkerung nach Nationalitätengruppen (in Prozent).

Tab. 9.4: Berufliche Stellung der erwerbstätigen Bevölkerung nach Einwanderungskohorten (in Prozent)

Tab. 9.5: Erwerbstätige Bevölkerung nach Wirtschaftszweigen und Nationalitätengruppen (in Prozent)

Tab. 9.6: Erwerbstätige Bevölkerung nach Wirtschaftszweigen und Einwanderungskohorten (in Prozent)

Tab. 9.7: Erwerbsbeteiligung und Beschäftigungsgrad nach Nationalitätengruppen (in Prozent). 
Tab. 9.8: Erwerbsbeteiligung und Beschäftigungsgrad nach Einwanderungskohorten (in Prozent).

Tab. 9.9: Erwerbslosenquoten und Dauer der Erwerbslosigkeit nach Nationalitätengruppen (in Prozent)................................................... 151

Tab. 9.10: Erwerbslosenquoten und Dauer der Erwerbslosigkeit nach Einwanderungskohorten (in Prozent) ................................................. 152

Tab. 9.11: Berechnung der Anzahl Schuljahre (SCH) ................................. 159

Tab. 9.12: Modellspezifikation: Erwerbslosigkeitswahrscheinlichkeit........... 160

Tab. 9.13: Deskriptive Statistiken zur Beschäftigungsperformance ................ 161

Tab. 9.14: LR-Tests auf Parameterstabilität ....................................................... 162

Tab. 9.15: Probit-Regressionen zur Erwerbslosigkeitswahrscheinlichkeit,

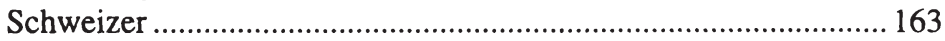

Tab. 9.16: Probit-Regressionen zur Erwerbslosigkeitswahrscheinlichkeit,

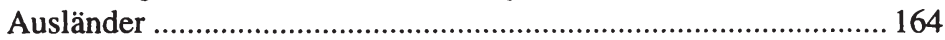

Tab. 9.18: Dissimilarity-Index D: Ergebnisse zur branchenspezifischen

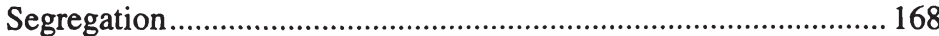

Tab. 10.1: Einkommenshöhe der erwerbstätigen Bevölkerung nach Nationalitätengruppen (in Prozent)....

Tab. 10.2: Einkommenshöhe der erwerbstätigen Bevölkerung nach Einwanderungskohorten (in Prozent)

Tab. 10.3: Modellspezifikation: Einkommensfunktion (ohne Heckman-

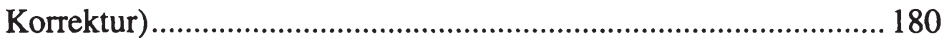

Tab. 10.4: Modellspezifikation: Heckman-Korrektur ..................................... 183

Tab. 10.5: Deskriptive Statistiken: Heckman-Korrektur.................................. 185

Tab. 10.6: Deskriptive Statistiken: Einkommensfunktion ............................... 186

Tab. 10.7: Chow-Test auf Parameterstabilität ................................................ 187

Tab. 10.8: OLS-Regressionen der Einkommensfunktion, Männer .................. 188

Tab. 10.9: Probit-Regressionen zur Erwerbstätigkeitswahrscheinlichkeit,

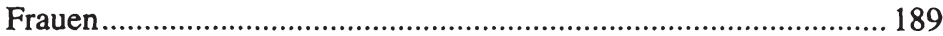

Tab. 10.10: OLS-Regressionen der Einkommensfunktion, Frauen................... 190

Tab. 10.11: Einkommenszerlegung Männer (Inländer - Ausländer) ………...... 196

Tab. 10.12: Einkommenszerlegung Frauen (Inländer - Ausländer) ................... 197

Tab. A8.2: Definition verschiedener Nationalitätengruppen............................ 212

Tab. A8.6: Einwanderungskohorten nach Nationalitätengruppen (in Prozent).

Tab. A8.7: Nationalitätengruppen nach Einwanderungskohorten (in Prozent).

Tab. A9.1: Höchste abgeschlossene Ausbildung der erwerbstätigen Bevölkerung nach Nationalitätengruppen (in Prozent).

Tab. A9.3: Berufliche Stellung der erwerbstätigen Bevölkerung nach Nationalitätengruppen (in Prozent) 
Tab. A9.5: Erwerbstätige Bevölkerung nach Wirtschaftszweigen und Nationalitätengruppen (in Prozent)

Tab. A9.7: Erwerbsbeteiligung und Beschäftigungsgrad nach Nationalitätengruppen (in Prozent) ............................................................... 218

Tab. A9.9: Erwerbslosenquoten und Dauer der Erwerbslosigkeit nach Nationalitätengruppen (in Prozent).................................................... 219

Tab. A9.17: Unterteilung der verschiedenen Branchen......................................220

Tab. A9.17: Unterteilung der verschiedenen Branchen (Fortsetzung) ............... 221

Tab. A9.18: Dissimilarity-Index zur Messung der beruflichen Segregation

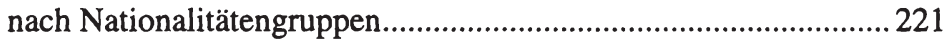

Tab. A10.1: Einkommenshöhe der erwerbstätigen Bevölkerung nach Nationalitätengruppen (in Prozent) ...................................................... 222 


\section{EINLEITUNG}

"After all that has been said of the levity and inconstancy of human nature, it appears evidently that a man is of all sorts of luggages the most difficult to be transported."

ADAM SMITH (1776): An Inquiry into the Nature and Causes of the Wealth of Nations

\subsection{Problemstellung}

\subsubsection{WaChSENDES MigRationspotential}

Die zunehmende Globalisierung und Öffnung der Märkte sowie die verstärkte Internationalisierung der Wirtschaftsbeziehungen in den vergangenen Jahren haben dazu geführt, dass sich die nationalen Volkswirtschaften einem immer grösseren Wettbewerbsdruck gegenübersehen, nicht nur auf den internationalen Güter- und Kapitalmärkten, sondern auch auf den Arbeitsmärkten. Um im internationalen Wettbewerb wirtschaftlich erfolgreich bestehen zu können, ist für diese Volkswirtschaften daher notwendig und wichtig, eine tragfähige Wirtschaftsverfassung $\mathrm{zu}$ schaffen und eine marktorientierte Wirtschaftspolitik zu verfolgen. Aus ökonomischer Sicht geht es somit im wesentlichen um die Implementierung attraktiver Standortfaktoren im internationalen Wettbewerb um die mobilen Produktionsfaktoren Arbeit und Kapital.

Internationale Wanderungsbewegungen spielen nicht nur hinsichtlich des internationalen Standortwettbewerbs eine wichtige Rolle. Die Bedeutung von Migration lässt sich auch anhand der Veränderungen in der Bevölkerungsstruktur der meisten westlichen Zuwanderungsländer veranschaulichen. Kennzeichnend in diesen Ländern ist die Überalterung der Bevölkerung, die das Ergebnis rückläufiger $\mathrm{Ge}-$ burten- und Sterberaten ist. Diese Entwicklung führt einerseits zu einer Verminderung der erwerbsfähigen Bevölkerung, womit sich die Frage stellt, ob die Arbeitsnachfrage zukünftig sowohl qualitativ als auch quantitativ befriedigend abgedeckt werden kann. Andererseits hat der Überalterungsprozess eine zunehmende finanzielle Belastung der erwerbstätigen Bevölkerung zur Folge, da die Alterssicherung in den meisten westlichen Industriestaaten zumindest teilweise im Rahmen umlagefinanzierter Systeme erfolgt [siehe GOLDER (1997a)].

Zuwanderung ist jedoch nicht nur mit Chancen, sondern auch mit Problemen für die Zielländer verbunden. Wirtschaftliche, politische, demographische und ökolo- 
gische Faktoren werden nicht nur zu einem verstärkten Migrationsdruck aus dem Süden, sondern vor allem auch aus dem Osten führen. Dieses Zuwanderungspotential stellt die Zielländer gleichermassen vor ein allokatives Arbeitsmarktproblem (wirtschaftlicher Faktor), ein Distributionsproblem (sozialer Faktor) sowie ein "Überfremdungsproblem" (politischer Faktor). Vor dem Hintergrund dieser Chancen und Probleme ist ein Überdenken der bestehenden Migrationspolitik dringend notwendig.

Als Reaktion auf den Komplex ungelöster Probleme sowie aufgrund der durch die Globalisierung bedingten Herausforderungen, werden Ausländer in den Gastländern heute subjektiv als Bedrohung empfunden. Eine Versachlichung der ausländerpolitischen Diskussion, die auf fundierten empirischen Ergebnissen basiert, erscheint daher unerlässlich. Diese Überlegungen bilden gleichzeitig Hintergrund und Anstoss zu der vorliegenden Studie über die Arbeitsmarktperformance von Ausländern in der Schweiz.

\subsubsection{HANDLUNGSBEDARF FÜR DIE SCHWEIZ}

Für die Schweiz sind die oben skizzierten Probleme und Herausforderungen wegen ihrer starken aussenwirtschaftlichen Verflechtungen von besonderer Bedeutung. Bereits heute weist sie, zusammen mit Luxemburg und Australien, von allen OECD Ländern den höchsten Ausländeranteil auf. Dieser belief sich im Jahre 1995 auf rund 19 Prozent. Noch höher fiel der ausländische Beschäftigungsanteil aus, der unter Berücksichtigung der Grenzgänger und Saisonniers im Jahre 1995 rund 34 Prozent betrug. Da die Schweiz eine vergleichsweise geringe Arbeitslosenquote und ein hohes Lohnniveau aufweist, ist sie eine attraktive Destination für Arbeitsuchende aus ärmeren Ländern. Es ist aus diesen Gründen zu vermuten, dass sich der Migrationsdruck in den kommenden Jahren weiter verstärken wird. Daraus ergibt sich sowohl innen- als auch aussenpolitischer Handlungsbedarf. ${ }^{1}$ Einerseits besteht die Notwendigkeit, die Einwanderungs- und Asylpolitik mit den Ländern der Europäischen Union zu koordinieren bzw. zu harmonisieren. Andererseits gilt es die geltende Ausländerpolitik in Richtung einer umfassenden Migrationspolitik zu revidieren und transparente Regeln für die Zuwanderung zu schaffen.

Gleichzeitig wird sich die Nachfrage nach Zuwanderern in der Schweiz aufgrund des ausgeprägten Alterungsprozesses auf der einen sowie des Mangels an hochqualifizierten Arbeitskräften auf der anderen Seite verstärken. Um den Bedürfnissen des Arbeitsmarktes adäquat Rechnung tragen zu können, ist daher eine $\mathrm{Zu}$ wanderungspolitik notwendig, die sowohl eine qualitative als auch quantitative Befriedigung der Arbeitsnachfrage gewährleistet. Vor dem Hintergrund dieses

Siehe EXPERTENKOMMISSION MigRATION (1997), Golder (1997b) und STRAUBHAAR (1989, 1991). 
Anpassungsbedarfes sowie als wichtige Vorbedingung für die Durchführung von Reformen ist eine fundierte Kenntnis der Performance der Einwanderer auf dem schweizerischen Arbeitsmarkt erforderlich. Die Überlegungen des vorangegangenen Abschnitts haben gezeigt, dass zwischen der Performance der Einwanderer und dem Wohlfahrtsniveau ein positiver Zusammenhang besteht. Diesen Aspekt gilt es, bei der Formulierung der Zulassungspolitik zu berücksichtigen. Wie aus internationalen empirischen Studien hervorgeht, bestehen zwischen Einwanderern aus unterschiedlichen Herkunftsländern z.T. substantielle Unterschiede in der Performance. ${ }^{2}$ Eine differenzierte Vorgehensweise bei der Analyse der Arbeitsmarktperformance ist daher unerlässlich, um aussagekräftige Ergebnisse zu ermöglichen.

Zusammenfassend lässt sich festhalten, dass die empirische Analyse der Performance von Einwanderern der Erweiterung des Informationsstandes dient und damit bei der Planung und Evaluation von Politikinstrumenten hilft. Dieser Aspekt ist für die Schweiz insbesondere deshalb von Bedeutung, weil der schweizerischen Ausländerpolitik vor allem ökonomische Überlegungen zugrunde liegen. Aus diesem Grund kommt der Frage nach der Effektivität der bestehenden Politik eine grosse Bedeutung zu. Gleichzeitig erlauben es die empirischen Ergebnisse, dem emotionsgeladenen Thema der Ausländerpolitik mit fundierten ökonomischen Argumenten zu begegnen. Die Zielsetzung dieser Studie besteht darin, einen Beitrag zur Versachlichung der schweizerischen Ausländerpolitik zu leisten. Aus wissenschaftlicher Sicht besteht der Hauptbeitrag der vorliegenden Studie in einer erstmaligen und detaillierten Untersuchung der Performance von Einwanderern in der Schweiz. Diese Thematik, die in der Migrationsforschung einen zentralen Stellenwert besitzt, wurde im schweizerischen Kontext bislang weitgehend vernachlässigt. ${ }^{3}$

\subsection{BEgrifFSDEFINITIONEN UND AUFBAu DER STUDIE}

\subsubsection{BEGRIFFSDEFINITIONEN}

Nach der Diskussion der Problemstellung steht in diesem Abschnitt die Erläuterung des Analysegegenstandes im Vordergrund. Einerseits werden die zentralen Termini dieser Studie erläutert. Andererseits soll die Relevanz des Untersu-

2 Empirische Evidenz zu Kanada findet sich z.B. in BEGGS/CHAPMAN (1991), zu den Vereinigten Staaten in BORJAS $(1992 b, 1991 b, 1988,1985)$ und CHISWICK $(1991,1988,1980)$ sowie für Deutschland in SCHMIDT $(1992,1994,1997)$.

3 Nach Wissen des Autors existiert bislang lediglich eine Studie für die Schweiz, die sich mit der Performance von Einwanderern befasst [MAECHLER (1995)]. Diese Studie beschränkt sich allerdings auf die Diskussion eines Teilaspektes der Arbeitsmarktperformance und ist zudem durch ein hohes Aggregationsniveau der Analyse charakterisiert. 
chungsgegenstandes für die wissenschaftliche und wirtschaftspolitische Diskussion verdeutlicht werden. Im speziellen soll geklärt werden, was unter dem Begriff der Arbeitsmarktperformance zu verstehen ist, und wie sie gemessen wird.

Die Analyse bzw. Messung der Arbeitsmarktperformance umfasst zwei komplementäre und interdependente Ansätze: die Beschäftigungs- und die Einkommensperformance. Im Zentrum steht dabei die Frage, wie erfolgreich die Ausländer hinsichtlich der Erwerbsbeteiligung bzw. Erwerbslosigkeit, der beruflichen Stellung, der Beschäftigung nach Wirtschaftszweigen sowie der Einkommenshöhe auf dem schweizerischen Arbeitsmarkt sind. Im Rahmen der empirischen Untersuchung werden dabei nicht nur produktivitätsbezogene, sondern auch diskriminierungsbedingte Faktoren berücksichtigt. Als Massstab bzw. Referenzgruppe zur Beurteilung der Performance der Einwanderer dient die Performance der schweizerischen Bevölkerung.

Die Analyse dieser beiden Themenkomplexe gibt nicht nur Aufschluss über den Erfolg der Ausländer auf dem schweizerischen Arbeitsmarkt, sondern vermag auch Erklärungen für allfällige Performanceunterschiede zu geben. Schliesslich ergeben sich aus der Analyse der Arbeitsmarktperformance auch Hinweise auf die allokativen und distributiven Wirkungen der Migration, d.h. auf die Effekte der Zuwanderung auf die Erwerbslage der Schweizer sowie auf das staatliche Umverteilungssystem. ${ }^{4}$ Die empirischen Ergebnisse zur Arbeitsmarktperformance sind daher insbesondere für Politiker von Bedeutung, da sich daraus Rückschlüsse auf das ökonomische Wohlergehen, das Armutsniveau, den Bezug öffentlicher Transfers sowie das Steueraufkommen ziehen lassen.

Die empirische Analyse der vorliegenden Studie beruht weitgehend auf den Daten der Schweizerischen Arbeitskräfteerhebung (SAKE). Die SAKE ist eine Stichprobenerhebung, die auf Befragungen der ständigen Wohnbevölkerung beruht. ${ }^{5}$ Diese umfasst allerdings nicht alle in der Schweiz erwerbstätigen Ausländer, sondern lediglich jene mit einer Jahres- oder Niederlassungsbewilligung. ${ }^{6}$ Diesen Umstand gilt es, bei der Interpretation der empirischen Ergebnisse zu berücksichtigen.

4 Eine Analyse der allokativen Effekte der Migration für die Schweiz findet sich in BLATTNER/SCHWARZ/SHELDON (1985), BLATTNER/SHELDON (1989), BÜRGENMEIER/BUTARE/FAVARGER (1992), KOHLI (1993, 1997), ROSSI/LEIGHTON (1971) SOwie SCHWARZ (1985, 1988). Eine Analyse der distributiven Effekte der Migration findet sich in STRAUBHAAR/WEBER (1994), WEBER (1993) sowie WEBER/STRAUBHAAR (1994).

5 Eine ausführliche Diskussion der empirischen Datenbasis findet sich in Abschnitt 8.4.

6 Eine Beschreibung der verschiedenen Aufenthaltskategorien findet sich in TAB. 2.3. 


\subsubsection{AUfBaU DER STUdie UND WiChTIGSTE ERGEBNISSE}

Die vorliegende Studie gliedert sich in drei Teile. Der erste Teil bietet einen Überblick $\mathrm{zu}$ den schweizerischen Migrationserfahrungen. Kapitel 2 gibt eine kurze historische Zusammenfassung der Migrationserfahrungen der Schweiz sowie der damit verbundenen Entwicklung der schweizerischen Migrationspolitik. Gezeigt wird, dass die Entwicklung der schweizerischen Volkswirtschaft in den vergangenen 150 Jahren seit der Gründung des schweizerischen Bundesstaates sehr stark von der Zuwanderung ausländischer Arbeitskräfte geprägt war. Als Folge des zunehmenden Ausländeranteils hat seit Mitte der 60er Jahre eine substantielle Verschärfung der schweizerischen Einwanderungsbestimmungen stattgefunden.

Kapitel 3 gibt einen Überblick über die wichtigsten sozio-demographischen Charakteristika der in Schweiz lebenden Ausländer. Diese Betrachtung vermittelt bereits erste Anhaltspunkte über die Arbeitsmarktperformance der In- und Ausländer. Die stilisierten, deskriptiven Statistiken dieses Kapitels zeigen, dass zwischen In- und Ausländern sowohl hinsichtlich der Beschäftigungs- als auch der Einkommensperformance substantielle Unterschiede bestehen. So weisen die Ausländer im Mittel nicht nur eine signifikant höhere Arbeitslosenquote, sondern auch ein tieferes Einkommensniveau auf.

Im zweiten Teil werden auf der Grundlage ökonomischer Theorien die wichtigsten Determinanten der Arbeitsmarktperformance erörtert. In Kapitel 4 stehen die bedeutendsten ökonomischen Ansätze zur Erklärung von Migration im Vordergrund. Es handelt sich dabei im wesentlichen um angebotsorientierte Theorien, über die sich das Entstehen eines Migrationspotentials erklären lässt. Während im Rahmen des neoklassischen sowie des Humankapitalansatzes das Individuum als Entscheidungsträger im Vordergrund steht, wird im Rahmen der Ansätze der Familienund Netzwerkmigration das Kollektiv als Entscheidungsträger betont.

In Kapitel 5 und 6 werden anschliessend die Bestimmungsfaktoren der Arbeitsmarktperformance erörtert. In Kapitel 5 steht die Analyse der produktivitätsbezogenen Determinanten der Arbeitsmarktperformance im Zentrum. Gemeint sind damit sowohl personenspezifische Attribute wie die Humankapitalausstattung als auch ziellandspezifische Faktoren wie die Arbeitsnachfrage oder die gesetzlichen Zuwanderungsbestimmungen. Die Analyse beschränkt sich dabei nicht auf die Analyse statistischer Aspekte, sondern trägt auch dynamischen Einflüssen Rechnung.

Während in Kapitel 5 produktivitätsbezogene Determinanten der Arbeitsmarktperformance im Zentrum des Interesses stehen, richtet sich das Hauptaugenmerk in Kapitel 6 auf den Einfluss diskriminatorischer Faktoren zur Erklärung der Arbeitsmarktperformance. Diskriminierung kann auf arbeitgeberseitiges Verhalten zurückgeführt werden aber auch institutionell bedingt sein. Im Zentrum der Analyse stehen die beiden interdependenten Ansätze der Einkommensdiskriminierung 
und der Arbeitsmarktsegmentation. Als Synthese der Ergebnisse der deskriptiven und theoretischen Analyse werden in Kapitel 7 schliesslich einige Hypothesen formuliert, die es im Rahmen der empirischen Analyse zu überprüfen gilt.

Im dritten Teil steht schliesslich die umfassende empirische Analyse der Arbeitsmarktperformance im Vordergrund. Die Messung der Arbeitsmarktperformance erfolgt im Rahmen der vorliegenden Studie über die Beschäftigungs- und die Einkommensperformance. Von Interesse ist in diesem Zusammenhang nicht nur der Vergleich der Performance von In- und Ausländern, sondern auch die Erörterung der Determinanten der Performance. Insbesondere gilt es zu untersuchen, welchen Erklärungswert produktivitätsbezogene im Vergleich zu diskriminierungsbedingten Faktoren besitzen. In Kapitel 8 werden die Untersuchungsmethode sowie die empirische Datenbasis erläutert. Dabei wird die Notwendigkeit einer differenzierten Vorgehensweise bei der empirischen Analyse hervorgehoben und dem Umstand Rechnung getragen, dass zwischen Einwanderern unterschiedlicher Nationalitätengruppen bzw. Einwanderungskohorten z.T. substantielle Divergenzen in den sozio-ökonomischen und sozio-demographischen Merkmalen bestehen.

In Kapitel 9 folgt die Evaluation der Beschäftigungsperformance, unter Berücksichtigung produktivitätsbezogener und diskriminierungsbedingter Faktoren. Die Determinanten der Erwerbstätigkeit bzw. Erwerbslosigkeit werden mittels deskriptiver Statistiken sowie unter Verwendung von Probit-Regressionen erörtert. Daneben wird auch auf den Einfluss der Arbeitsmarktsegmentation bei der Erklärung der Beschäftigungsperformance eingegangen. Die empirische Analyse zeigt, dass Ausländer im Mittel eine höhere Erwerbslosigkeitswahrscheinlichkeit besitzen als Einheimische. Gleichzeitig geht aus der Analyse hervor, dass geschlechtsund nationalitätengruppenspezifische Unterschiede bestehen. Diese Unterschiede spiegeln sich auch im Ausmass der Arbeitsmarktsegmentation wider.

In Kapitel 10 folgt schliesslich die empirische Analyse der Einkommensperformance. Analog zur Analyse der Beschäftigungsperformance wird auch hier neben den produktivitätsbezogenen Faktoren der Möglichkeit diskriminierenden Verhaltens Rechnung getragen. Die empirischen Ergebnisse zeigen, dass Männer sowohl bei den In- als auch bei den Ausländern deutlich höhere Einkommen erzielen als Frauen. Die Berücksichtigung der verschiedenen Nationalitätengruppen zeigt, dass die Performance der Nordeuropäer am besten ausfällt und dabei sogar jene der Inländer übertrifft. Die Zerlegung der Einkommensdifferentiale lässt schliesslich erkennen, dass dem Ausstattungseffekt eine geringere Bedeutung als dem Diskriminierungseffekt zukommt.

Auf der Grundlage der theoretischen und empirischen Ergebnisse der vorliegenden Studie werden in Kapitel 11 schliesslich einige wirtschaftspolitische Schlussfolgerungen gezogen. Diese beziehen sich im wesentlichen auf die Option einer Neuorientierung der schweizerischen Migrationspolitik, um den einleitend beschriebenen Herausforderungen der Zukunft angemessen Rechnung tragen $\mathrm{zu}$ 
können. Es zeigt sich dabei, dass dazu ein Übergang von der bestehenden DreiKreise-Politik zur Zwei-Kreise-Politik vollzogen werden müsste.

\subsection{EINGRENZUNG DES UNTERSUCHUNGSGEGENSTANDES}

Angesichts des weltweit steigenden Migrationspotentials, besitzt die Migrationsforschung bei der Bewältigung der zukünftigen politischen, sozialen, demographischen und wirtschaftlichen Herausforderungen grosse Bedeutung. Es gilt allerdings zu beachten, dass Wanderungsbewegungen nicht als eindimensionales Phänomen verstanden werden können, da sie alle Bereiche der gesellschaftlichen Organisation einer Volkswirtschaft tangieren. Im Bewusstsein der Mehrdimensionalität der Zuwanderung erfolgt die Analyse der Arbeitsmarktperformance jedoch aus rein ökonomischer Perspektive und somit auf dem Menschenbild des sog. 'homo oeconomicus' aufbauend, das dem Idealtyp des rational handelnden, nutzenmaximierenden Individuums entspricht. Unberücksichtigt bleiben daher diejenigen sozialwissenschaftlichen Ansätze, die von anderen Grundannahmen ausgehen.

Die damit verbundenen Probleme lassen sich anhand des in dieser Studie verwendeten Begriffs der Assimilation veranschaulichen, der hier als Anpassungsprozess der Beschäftigungs- und Einkommensprofile der Einwanderer an jene der Einheimischen verstanden wird. Die soziologischen und psychologischen Aspekte dieses Prozesses werden dabei im Rahmen der vorliegenden Analyse weitgehend ausgeklammert. Die Evaluation der Arbeitsmarktperformance erfolgt somit bewusst aus der 'ökonomischen Perspektive', die damit gleichsam ein zentrales Problem der Migrationsforschung offenlegt, nämlich das Fehlen einer umfassenden, interdisziplinären Theorie der Migration. ${ }^{7}$ Der ökonomische Fokus der vorliegenden Studie führt dazu, dass eine Reihe wichtiger Aspekte vernachlässigt bleiben. Gleichzeitig eröffnet die Konzentration auf ökonomische Aspekte jedoch die Möglichkeit präziserer Aussagen in diesem Teilbereich, die sich zudem empirisch überprüfen lassen.

7 In neuerer Zeit hat allerdings der sog. Systemansatz der Migration, verstärkt an Bedeutung gewonnen. Dieser Ansatz basiert auf der Annahme interdependenter Beziehungen innerhalb des globalen Systems, die sich gegenseitig stabilisieren, so dass langfristig ein Gleichgewicht gewährleistet ist. Elemente dieses Systems, das länderübergreifend zu verstehen ist und von verschiedenen gesellschaftlichen Subsystemen getragen wird, sind sowohl nationale Politiken als auch wirtschaftliche Handlungsträger. Der Systemansatz hebt die Interdependenz von politischen, sozialen und wirtschaftlichen Faktoren zur Bestimmung von Migrationflüssen hervor. Der Vorteil des Systemansatzes besteht darin, dass aufgrund der Erweiterung des Spektrums möglicher Migrationsursachen eine umfassende Darstellung der Interdependenzen, die zu Migration führen können, möglich ist. Der Nachteil des Systemansatzes liegt in der Schwierigkeit, die einzelnen Einflussfaktoren zu isolieren und damit in seiner Operationalisierung [siehe BOYD (1989), FAWCETT (1989), FAWCETT/ARNOLD (1987) sowie PORTES/BACH (1985)]. 
Stefan Golder - 978-3-631-74998-2

Downloaded from PubFactory at 01/11/2019 09:25:33AM

via free access 


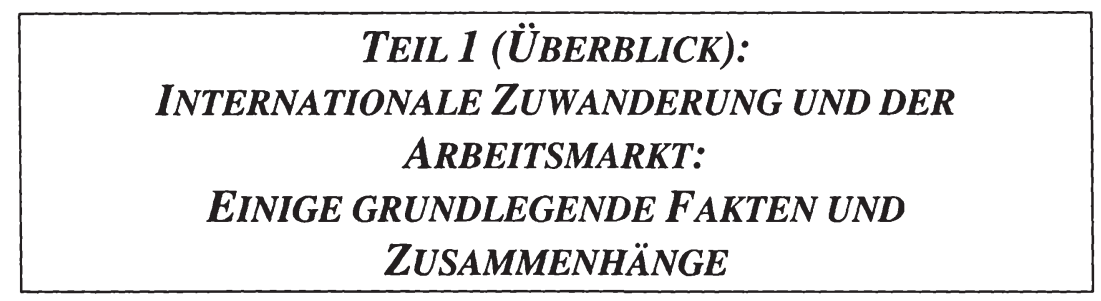

\section{MIGRATIONSERFAHRUNGEN DER SCHWEIZ - HISTORISCHER ÜBERBLICK VOR DEM HINTERGRUND DER ENTWICKLUNG AUF DEM} ARBEITSMARKT

Ziel dieses Kapitels ist es, einen historischen Überblick über die Zuwanderung in die Schweiz zu geben sowie über die damit zusammenhängenden Veränderungen der Zielsetzungen und Instrumente der schweizerischen Migrationspolitik. Im Zentrum steht dabei die Frage nach dem Einfluss dieser Veränderungen auf die Arbeitsmarktperformance der Einwanderer.

Die Entwicklung der schweizerischen Volkswirtschaft seit der Gründung des Bundesstaates ist stark von der Zuwanderung ausländischer Arbeitskräfte geprägt. Seit 1850 hat sich der Anteil der ausländischen Bevölkerung von rund 3 Prozent auf über 19 Prozent mehr als versechsfacht, so dass zum heutigen Zeitpunkt nahezu jeder dritte Beschäftigte in der Schweiz ausländischer Herkunft ist.

Nach einer Periode vollständiger Freizügigkeit und Niederlassungsfreiheit zu Beginn dieses Jahrhunderts, die zu einem ersten Höchststand des Ausländeranteils von rund 15 Prozent führte, wurde die Einwanderungspolitik bis zum Ende des Zweiten Weltkriegs sehr restriktiv gehandhabt. Der Aufschwung der Nachkriegsjahre und der damit verbundene Arbeitskräftebedarf führte wiederum zu einer Phase liberaler Zulassungspraxis. Überhitzungserscheinungen sowie Ängste vor drohender Überfremdung veranlassten die Regierung gegen Mitte der 60er Jahre, die Zuwanderung ausländischer Arbeitskräfte quantitativ zu beschränken. Diese Kontingentierungspraxis findet auch heute noch Anwendung.

Während die Zuwanderung der ersten Nachkriegsjahre eine vergleichbare Struktur zu jener zu Beginn dieses Jahrhunderts aufwies, haben seither substantielle Veränderungen hinsichtlich der Herkunftsländer und Migrationsmotive stattgefunden. Die wirtschaftlichen Herausforderungen sowie die damaligen Schwächen der Migrationspolitik haben seit Beginn der 90er Jahre zu einer substantiellen Neuorientierung der schweizerischen Migrationspolitik geführt. 


\subsection{LibERALE ZuLASSUNGSPOLITIK}

Die Phase der liberalen Zulassungspolitik lässt sich in zwei Zeitabschnitte unterteilen. Mit dem Einsetzen des Industrialisierungsprozesses gegen Mitte des 18. Jahrhunderts begann die erste Phase liberaler Zulassungspolitik, die bis zum Ausbruch des Ersten Weltkriegs dauerte. Mit dem Ende des Zweiten Weltkriegs setzte die zweite Phase ein, die bis zur Einführung von Zuwanderungsbeschränkungen im Jahre 1963 Bestand hatte.

\subsubsection{DIE ZEIT VOR 1945}

Im Verhältnis zu ihrer wirtschaftlichen Leistungsfähigkeit war die Schweiz lange Zeit überbevölkert. Dies führte bis gegen Ende des 19. Jahrhunderts zu grossen Auswanderungsbewegungen. Wirtschaftliche und soziale Not, vor allem der bäuerlichen Bevölkerung, waren die Triebfedern der Massenauswanderungen im 19. Jahrhundert, die im Jahre 1854 mit rund 18'000 Personen oder 0.7 Prozent der Wohnbevölkerung ihren Höhepunkt erreichten. ${ }^{8}$ Das Fehlen wirtschaftlicher Anreize sowie die restriktiven Einwanderungsbestimmungen der Kantone und Kommunen führten dazu, dass bis Mitte des 19. Jahrhunderts nur sehr geringfügige Zuwanderungsbewegungen stattfanden. ${ }^{9}$

Erst mit dem Industrialisierungsprozess setzte ein nennenswerter Bedarf an ausländischen Arbeitskräften ein. Die im Vergleich zum Ausland attraktiveren Arbeitsverhältnisse sowie die vollständige Personenfreizügigkeit führten ab Mitte des 19. Jahrhunderts zu einer stetigen Zuwanderung ausländischer Arbeitskräfte. Im Jahre 1914 erreichte der Ausländerbestand mit 600'000 Personen bzw. 15.4 Prozent der Gesamtbevölkerung einen ersten Höchststand (siehe TAB. 2.1). ${ }^{10}$

Bis zum Beginn des 20. Jahrhunderts fiel die Regelung des Aufenthalts und der Niederlassung von Ausländern in die ausschliessliche Zuständigkeit der Kantone. ${ }^{1}$ Deren Handlungsspielraum war jedoch durch bilaterale Niederlassungsverträge bzw. Niederlassungsvereinbarungen eingeschränkt. Angehörige von Staaten mit denen solche Vereinbarungen bestanden, konnten sich ohne Einschränkungen in der Schweiz niederlassen und eine Erwerbstätigkeit aufnehmen. Diese Freizügigkeitspraxis wurde i.d.R. auch gegenüber Ausländern angewandt,

8 LEHMANN (1949). Zwischen dem 16. und 18. Jahrhundert wanderten zwischen 300'000 und 350'000 Schweizer aus, um als Söldner in ausländischen Heeren Dienst zu leisten [BICKEL (1947)].

9 HOFFMANN-NOWOTNY (1985).

10 HOFFMANN-NOWOTNY/KILIAS (1979).

11 Der Bund war gemäss Artikel $70 \mathrm{BV}$ nur dann befugt Ausländer auszuweisen, wenn diese entweder vorbestraft waren, der Fürsorge zur Last zu fallen drohten oder politisch als gefährlich erschienen [BIGA (1964)]. 
mit deren Heimatstaaten keine Niederlassungsverträge existierten. ${ }^{12}$ De facto bestand während dieser Periode somit zwischen Schweizern und Ausländern der einzige Unterschied darin, dass die Ausländer über keine politischen Rechte verfügten.

Tab. 2.1: Entwicklung des Ausländerbestandes in der Schweiz, 1850-1990

\begin{tabular}{|c|cccc|}
\hline Jahr & $\begin{array}{c}\text { Gesamtbevölke- } \\
\text { rung (in 1'000) }\end{array}$ & $\begin{array}{c}\text { Schweizer } \\
\text { (in 1'000) }\end{array}$ & $\begin{array}{c}\text { Ausländer } \\
\text { (in 1'000) }\end{array}$ & $\begin{array}{c}\text { Ausländeranteil } \\
\text { (in \%) }\end{array}$ \\
\hline 1850 & $2^{\prime} 393$ & $2^{\prime} 321$ & 72 & 3.0 \\
1860 & $2^{\prime} 510$ & $2^{\prime} 396$ & 115 & 4.6 \\
1870 & $2^{\prime} 655$ & $2^{\prime} 518$ & 151 & 5.7 \\
1880 & $2^{\prime} 832$ & $2^{\prime} 635$ & 211 & 7.5 \\
1890 & $2^{\prime} 918$ & $2^{\prime} 688$ & 230 & 7.9 \\
1900 & $3^{\prime} 315$ & $2^{\prime} 932$ & 383 & 11.6 \\
1910 & $3^{\prime} 753$ & $3^{\prime} 201$ & 552 & 14.7 \\
1920 & $3^{\prime} 880$ & $3^{\prime} 478$ & 402 & 10.4 \\
1930 & $4^{\prime} 066$ & $3^{\prime} 711$ & 356 & 8.7 \\
1940 & $4^{\prime} 266$ & $4^{\prime} 042$ & 224 & 5.2 \\
1950 & $4^{\prime} 715$ & $4^{\prime} 430$ & 285 & 6.1 \\
1960 & $5^{\prime} 429$ & $4^{\prime} 844$ & 585 & 10.8 \\
1970 & $6^{\prime} 270$ & $5^{\prime} 190$ & $1^{\prime} 080$ & 17.2 \\
1980 & $6^{\prime} 366$ & $5^{\prime} 421$ & 945 & 14.8 \\
1990 & $6^{\prime} 874$ & $5^{\prime} 628$ & $1^{\prime} 245$ & 18.1 \\
\hline
\end{tabular}

Quelle: BFS (diverse Jahrgänge), eigene Berechnungen

Die massive Zunahme der ausländischen Bevölkerung löste in der schweizerischen Bevölkerung zunehmendes Unbehagen aus. Dieser Phase entstammt der auch heute noch weitverbreitete Begriff der 'Überfremdung' ${ }^{13}$ Es ist in diesem Zusammenhang von Interesse, dass die politischen Vorstösse zu Beginn dieses Jahrhunderts, im Gegensatz zu jenen in den 60er und 70er Jahren, vor allem durch Forderungen nach verstärkter Integration und Naturalisierung der in der Schweiz lebenden Ausländer charakterisiert waren. So wurde z.B. die Einführung des sog. ius soli gefordert, demzufolge ein in der Schweiz geborenes Kind ausländischer Eltern automatisch die schweizerische Staatsbürgerschaft erhalten sollte.

Der Ausbruch des Ersten Weltkrieges setzte der Freizügigkeit jedoch ein Ende. Gleichzeitig begann der Bund erstmals im Ausländerrecht tätig zu werden. Er erliess, vorerst noch ohne verfassungsmässige Grundlage, verschiedene Verordnun-

$\begin{array}{ll}12 & \text { BIGA/BFA (1991). } \\ 13 & \text { SCHLAEPFER (1969). }\end{array}$ 
gen, die einerseits die Einführung von Grenzkontrollen beinhalteten, andererseits aber gleichzeitig der Begrenzung der Zahl der Ausländer sowie der Bekämpfung der Arbeitslosigkeit dienen sollten. ${ }^{14}$ Der Krieg sowie die restriktive Zulassungspolitik führten ab 1915 zu einem markanten Rückgang der ausländischen Wohnbevölkerung.

Die zunehmende Bedeutung der Themenkomplexe Arbeitslosigkeit und Ausländeranteil hatten eine immer stärkere Kompetenzverlagerung von den Kantonen zum Bund zur Folge, die mit der Aufnahme von Art. $69^{\text {ter }}$ in die Bundesverfassung im Jahre 1925 ihren Abschluss fand. Diese Kompetenzverschiebung wurde zusätzlich durch die Tendenz des Bundes akzentuiert, die Entscheidungsspielräume der Kantone durch materielle Vorgaben einzuschränken.

Tab. 2.2: Art. $69^{\text {ter }} B V$

\footnotetext{
1 Die Gesetzgebung über Ein- und Ausreise, Aufenthalt und Niederlassung der Ausländer steht dem Bund zu.

2 Die Entscheidung über Aufenthalt und Niederlassung treffen nach Massgabe des Bundesrechtes die Kantone. Dem Bund steht jedoch das endgültige Entscheidungsrecht zu gegenüber:

a. kantonale Bewilligungen für länger dauernden Aufenthalt, für Niederlassung und gegenüber Toleranzbewilligungen;

b. Verletzung der Niederlassungsverträge;

c. kantonale Ausweisungen aus dem Gebiete der Eidgenossenschaft;

d. Verweigerung des Asyls.
}

Auf der Basis von Art. $69^{\text {ter }}$ erliess der Bundesrat im Jahre 1931 das Bundesgesetz über die Niederlassung und den Aufenthalt von Ausländern (ANAG). Das ANAG wurde als Rahmengesetz konzipiert und enthält nur sehr wenige materielle Vorschriften. Es weist dem Bundesrat umfangreiche Kompetenzen zu, von denen dieser auch Gebrauch gemacht und zahlreiche Verordnungen erlassen hat, die wesentliche Aspekte des Fremdenpolizeirechts regeln. $\mathrm{Zu}$ den wichtigsten Verordnungen zählen die Vollziehungsverordnung vom 1. März 1949 zum Bundesgesetz über den Aufenthalt und die Niederlassung von Ausländern (ANAV) sowie die Verordnung vom 6. Oktober 1986 über die Begrenzung der Zahl der Ausländer (BVO).

Ausländerrecht ist primär Polizeirecht, d.h. Teil der staatlichen Tätigkeit zum Schutze der öffentlichen Ruhe und Ordnung sowie der öffentlichen Sicherheit. Das Ausländerrecht verfolgt verschiedene Zielsetzungen. Zum einen sollen unerwünschte Personen an der Einreise und dem Aufenthalt in der Schweiz gehindert werden. Zum anderen dient es aber auch als Instrument zur Stabilisierung des Arbeitsmarktes, zur Bekämpfung der Arbeitslosigkeit und zur Steuerung des Aus-

14 BIGA/BFA (1991: 17), KÄLIN/RIEDER (1997). 
länderanteils an der Gesamtbevölkerung. ${ }^{15}$ Diese Zielsetzungen in Verbindung mit der Weltwirtschaftskrise der 30er Jahre und dem Kriegsausbruch führten in der Folge zu einer Verschärfung der Zulassungsbestimmungen, so dass der Ausländeranteil im Jahre 1941 einen Tiefstand in diesem Jahrhundert von 224'000 Personen oder 5.2 Prozent der Gesamtbevölkerung erreichte.

\section{Tab. 2.3: Die Bewilligungskategorien des schweizerischen Ausländerrechts}

\begin{tabular}{|c|c|}
\hline $\begin{array}{l}\text { Jahresaufenthaltsbewilligung (B-Ausweis) } \\
\text { - Allgemeine Bewilligungsart } \\
\text { - Nicht dauernd zugelassene Ausländer/innen } \\
\text { - Aufenthalt auf ein Jahr befristet } \\
\text { - Jährliche Verlängerung möglich; ab } 5 \text { Jahren } \\
\text { alle } 2 \text { Jahre } \\
\text { - Für Erwerbstätige kontingentiert } \\
\text { - Kann mit Bedingungen verbunden werden } \\
\text { - Recht auf sofortigen Familiennachzug, falls } \\
\text { Stelle gesichert und genügende Mittel bzw. } \\
\text { Wohnung vorhanden }\end{array}$ & $\begin{array}{l}\text { Niederlassungsbewilligung (C-Ausweis) } \\
\text { - } \text { Für dauernd zugelassene Ausländer/innen } \\
\text { nach } 5 \text { bis } 10 \text { Jahren Aufenthalt mit B- } \\
\text { Ausweis } \\
\text { - } \text { Unbefristet } \\
\text { - } \\
\text { Verlängerung nach } 3 \text { Jahren (Kontroll- } \\
\text { frist) } \\
\text { - } \\
\text { - } \\
\text { Nicht kontingentiert } \\
\text { - } \\
\text { Recht auf Familiennachzug } \\
\text { dige Arbeit }\end{array}$ \\
\hline $\begin{array}{l}\text { Kurzaufenthaltsbewilligung (L-Ausweis) } \\
\text { - Zu Weiterbildungszwecken oder zur } \\
\text { Abdeckung des Arbeitskräftebedarfs für kür- } \\
\text { zere Arbeiten } \\
\text { - Aufenthalt von } 4 \text { bis max. } 18 \text { Monaten } \\
\text { - Nicht verlängerbar } \\
\text { - Für die meisten Kategorien kontingentiert } \\
\text { - Kein Recht auf Familiennachzug }\end{array}$ & $\begin{array}{l}\text { Saisonnierbewilligung (A-Ausweis) } \\
\text { - } \quad \text { Arbeitnehmer in Saisonbetrieben } \\
\text { - } \text { Aufenthalt für max. } 9 \text { Monate pro Jahr (3 } \\
\text { Monate müssen ausserhalb der Schweiz } \\
\text { verbracht werden) } \\
\text { - } \text { Verlängerung möglich aber kein An- } \\
\text { spruch } \\
\text { - } \text { Kontingentiert } \\
\text { - } \text { Kein Recht auf Familiennachzug } \\
\text { - Nach } 4 \text { aufeinanderfolgenden Saisons } \\
\text { Möglichkeit der Umwandlung in Jahres- } \\
\text { aufenthaltsbewilligung }\end{array}$ \\
\hline $\begin{array}{l}\text { Grenzgängerbewilligung (G-Ausweis) } \\
\text { - Ausländer/innen die seit } 6 \text { Monaten in der } \\
\text { Grenzzone eines Nachbarlandes wohnen und } \\
\text { in der Schweiz arbeiten } \\
\text { - Tägliche Rückkehr an den Wohnort } \\
\text { - Jährliche Verlängerung } \\
\text { - Nicht kontingentiert } \\
\text { - Stellenwechsel nach dem ersten Jahr möglich }\end{array}$ & $\begin{array}{l}\text { Vorläufig Aufgenommene (F-Ausweis) } \\
\text { - Ausländer/innen, deren Weg- oder Aus- } \\
\text { weisung nicht möglich oder zumutbar ist } \\
\text { - Aufnahme für } 12 \text { Monate, jährliche Ver- } \\
\text { längerung } \\
\text { - Wird aufgehoben bei Wegfall der Voll- } \\
\text { zugshindernisse } \\
\text { - Kann gruppenweise verfügt werden } \\
\text { - Erwerbstätigkeit kann bewilligt werden } \\
\text { - Familiennachzug nur bei Erteilung einer } \\
\text { Jahresaufenthaltsbewilligung }\end{array}$ \\
\hline
\end{tabular}

Quelle: Kälin/Rieder (1997)

15 BERICHT DES BUNDESRATES (1991), BIGA/BFA (1991). 
Gemäss Art. 1 ANAG besteht für die Anwesenheit von Ausländern in der Schweiz eine Bewilligungspflicht. Diese Pflicht gilt unabhängig davon, ob diese einer Erwerbstätigkeit nachgehen wollen oder nicht. Bei der Beurteilung der jeweiligen Gesuche wird überprüft, ob die antragstellenden Personen die gesetzlichen Anforderungen erfüllen. Gemäss Art. 4 ANAG entscheiden die Behörden im Rahmen der gesetzlichen Bestimmungen und völkerrechtlichen Vereinbarungen nach freiem Ermessen über die Gewährung oder Verweigerung einer Bewilligung. Alle fremdenpolizeilichen Bewilligungen gelten dabei lediglich für denjenigen Kanton, der sie ausgestellt hat. Ausländer benötigen daher für den Kantonswechsel eine besondere Bewilligung. Nicht niedergelassene Ausländer müssen zudem eine Bewilligung für den Stellen- und Berufswechsel einholen. In TAB. 2.3 sind die verschiedenen Bewilligungskategorien des schweizerischen Ausländerrechts sowie die wichtigsten dazugehörenden Merkmale aufgelistet.

Das schweizerische Ausländerrecht ist dadurch gekennzeichnet, dass sich die Erteilung einer Aufenthaltsbewilligung grundsätzlich auf den Tatbestand der Erwerbstätigkeit stützt, d.h. der Gesetzgeber i.a. davon ausgeht, dass Ausländer zur Aufnahme einer Erwerbstätigkeit in die Schweiz einreisen. Trifft dies nicht zu, so handelt es sich entweder um Zuwanderung im Rahmen des Familiennachzugs oder um die Einreise von Asylsuchenden oder Gewalt- und Kriegsflüchtlingen. TAB. 2.4 gibt Aufschluss über die Dimension und Bedeutung der einzelnen $\mathrm{Zu}-$ wanderungsgruppen im Jahre 1997. Es gilt dabei allerdings zu beachten, dass diese Zahlen lediglich eine Momentaufnahme darstellen.

Tab. 2.4: Einreisen in die Schweiz nach Motiven, 1997

\begin{tabular}{|l|c|c|}
\hline Einreisen & in Prozent & Absolut \\
\hline - Familiennachzug & 14.0 & $22^{\prime} 412$ \\
- Erwerbstätigkeit mit Kontingent des & 8.6 & $1^{\prime} 777$ \\
Bundes und der Kantone & & \\
- Umwandlung von Saisonnierbewil- & 1.7 & $2^{\prime} 655$ \\
ligungen in Jahresbewilligungen & & \\
- Schüler, Studenten, Rentner und Er- & 6.2 & $9^{\prime} 908$ \\
holungsaufenthalter & & \\
- Humanitäre Gründe & 1.2 & $1^{\prime} 878$ \\
- Übrige & 12.7 & $20^{\prime 2} 261$ \\
- Saisonnier & 29.3 & $46^{\prime} 720$ \\
- Grenzgänger & 11.3 & $18^{\prime} 119$ \\
- Asylsuchende & 15.0 & $23^{\prime} 982$ \\
\hline
\end{tabular}

Quelle: BFA (1998), BFF (1998), eigene Berechnungen 
Die Tatsache, dass der Gesetzgeber von der Annahme ausgeht, dass Zuwanderung in der Regel zum Zweck der Erwerbstätigkeit erfolgt, spiegelt sich auch in der spezifischen Eigenart der schweizerischen Ausländergesetzgebung wider, die keine Trennung zwischen der Aufenthalts- und Arbeitserlaubnis kennt. Die oben dargestellten Bewilligungskategorien definieren damit sowohl den Aufenthaltsals auch den Arbeitsmarktstatus der Ausländer. Wie aus TAB. 2.3 hervorgeht, verbessert sich die Rechtsstellung der Ausländer auf dem schweizerischen Arbeitsmarkt mit zunehmender Aufenthaltsdauer.

\subsubsection{DIE NACHKRIEGSZEIT}

Die günstige Wirtschaftsentwicklung nach dem Zweiten Weltkrieg löste aufgrund des begrenzten einheimischen Arbeitskräftepotentials eine beträchtliche Nachfrage nach ausländischen Arbeitskräften aus. Die daraus resultierende liberale, primär nachfrageorientierte Zulassungspolitik führte in der Folge zu einer raschen $\mathrm{Zu}$ nahme des Ausländerbestandes [siehe TAB. 2.1]. ${ }^{16}$ Zwischen 1950 und 1960 erhöhte sich die Zahl der in der Schweiz wohnhaften Ausländer von $285^{\prime} 000$ auf $585^{\prime} 000$ Personen, was einer jährlichen Wachstumsrate von 7.4 Prozent entsprach. Die massive Vergrösserung der ausländischen Bevölkerung wurde anfänglich als konjunkturbedingtes und damit lediglich temporäres Phänomen des Nachkriegsbooms verstanden.

Diese Annahme beruhte im wesentlichen auf zwei Überlegungen: der Rotationsthese und der Konjunkturpufferfunktion ausländischer Arbeitnehmer. Der Rotationsthese lag die Auffassung zugrunde, dass ein typischer Einwanderer jung und unverheiratet sei und dessen Zielsetzung in erster Linie darin bestehe, Vermögen zu akkumulieren, um sich damit im Herkunftsland anschliessend eine eigene Existenz aufbauen zu können. Es konnte somit von einer temporären, auf wenige Jahre beschränkten Aufenthaltsdauer der ausländischen Arbeitskräfte ausgegangen und dementsprechend mit einer hohen Rotation des Ausländerbestandes gerechnet werden. Eng verbunden mit der Rotationsthese war die Vorstellung, dass die ausländischen Arbeitskräfte eine Konjunkturpufferfunktion übernehmen. Dieser lag die Überlegung zugrunde, dass sich das Arbeitslosigkeitsrisiko für schweizerische Arbeitskräfte in rezessiven Phasen durch die Kombination einer hohen Rotation ausländischer Arbeitskräfte mit restriktiven Zulassungsbestimmungen reduzieren liesse.

Während die Zuwanderung ausländischer Arbeitskräfte liberal gehandhabt wurde, war die geographische und berufliche Mobilität der ausländischen Arbeitskräfte

16 Die Nachfrage nach ausländischen Arbeitskräften konzentrierte sich zu jener Zeit vor allem auf diejenigen Branchen, aus denen Schweizer Arbeitskräfte mangels Attraktivität (Lohn, Arbeitsbedingungen, soziale Stellung) abgewandert waren. Dieser Umstand führte somit zu einer Konzentration der ausländischen Arbeitskräfte in Branchen mit chronischem $\mathrm{Ar}$ beitskräftemangel. 
eingeschränkt. Diese Massnahmen wurden ergriffen, um die Abwanderung ausländischer Arbeitskräfte in attraktivere Branchen und Regionen und die damit verbundene Notwendigkeit weiterer Zuwanderung zu verhindern. Aufenthaltsbewilligungen wurden i.d.R. nur für einen bestimmten Arbeitsplatz erteilt. Da der Stellen- und Berufswechsel bewilligungspflichtig war, bedeutete dies, dass der grösste Teil der ausländischen Arbeitskräfte geographisch und beruflich immobil war. Diese ausländerrechtlichen Bestimmungen führten zur Strukturerhaltung in unattraktiven Branchen und peripheren Regionen sowie zu einer Verbesserung der Arbeitsmarktperformance der schweizerischen Arbeitskräfte. Zusammenfassend lässt sich somit festhalten, dass in dieser Phase zwar die Zulassung ausländischer Arbeitskräfte liberal gehandhabt wurde, dass aber die Rechte der Ausländer stark eingeschränkt waren. ${ }^{17}$

\subsection{ReSTRIKTIVE ZULASSUNGSPOLITIK}

Wie die Phase liberaler Zulassungspolitik, lässt sich auch die Phase restriktiver Zulassungspolitik in zwei Zeitabschnitte unterteilen. Der erste Zeitabschnitt dauerte von 1963 bis 1970 und beinhaltete eine betriebsweise Plafonierung der Ausländerbeschäftigung. Der zweite Zeitabschnitt, der 1970 begann, ist durch eine Globalplafonierung gekennzeichnet.

\subsubsection{BetriebsWeise Plafonierung (1963 - 1970)}

Anfang der 60er Jahre wuchs der Bestand der ständigen Wohnbevölkerung infolge der anhaltenden Hochkonjunktur und der zunehmenden Bedeutung der Familienmigration um fast $90^{\prime} 000$ Personen jährlich. ${ }^{18}$ Diese Entwicklung führte zur Erkenntnis, dass viele Ausländer auf Dauer in der Schweiz bleiben würden und daher mit zunehmender Familienmigration gerechnet werden müsse. Hohe Zuwachsraten der in der Schweiz lebenden Ausländer in Verbindung mit konjunkturellen Überhitzungserscheinungen verstärkten die Forderungen nach staatlichen Interventionen und Dämpfungsmassnahmen.

Mit dem Bundesratsbeschluss (BRB) vom 1. März 1963 über die Beschränkung der Zulassung ausländischer Arbeitskräfte erfolgten erstmals seit Ende des Zweiten Weltkriegs staatliche Begrenzungsmassnahmen. Zur Kontrolle des Zustroms ausländischer Arbeitnehmer aus konjunkturpolitischen Gründen sowie zur Abwehr der "Überfremdungsgefahr" wurde der Ausländerbestand auf betrieblicher Ebene plafoniert. Anfänglich wurden neue Bewilligungen für erwerbstätige Ausländer sowie Bewilligungen für den Stellenwechsel nur noch dann erteilt, wenn der Gesamtpersonalbestand der jeweiligen Betriebe den Stand von Dezember

17 SCHWARZ (1988).

18 Von August 1960 bis August 1961 erhöhte sich die Zahl der kontrollpflichtigen Ausländer (Jahresaufenthalter, Grenzgänger und Saisonniers) um 112'836 Personen oder 25.9 Prozent. 
1962 nicht überstieg. Ab 1964 wurden diese Bewilligungen nur noch dann erteilt, wenn der Gesamtpersonalbestand zuvor um einen bestimmten Prozentsatz reduziert worden war.

Das Problem bestand aber nach wie vor darin, dass abgewanderte Schweizer durch Ausländer ersetzt werden konnten, was eine weitere Zunahme des Ausländerbestandes zur Folge hatte. Die Betriebe wurden daher ab 1965 zusätzlich verpflichtet, ihren Bestand an ausländischen Arbeitskräften um 5 Prozent zu reduzieren. Diese zusätzlich zur Gesamtplafonierung festgelegte Beschränkung sollte verhindern, dass die aus den Betrieben abgewanderten schweizerischen Arbeitskräfte durch ausländische ersetzt wurden.

Die ausländische Wohnbevölkerung wuchs aber weiterhin, insbesondere die Zahl der Jahresaufenthalter und Niedergelassenen. Die Hauptgründe für diese weitere Zunahme des Ausländerbestandes waren auf den beschränkten Geltungsbereich der Begrenzungsbestimmungen und die Existenz verschiedener Ausnahmebestimmungen zurückzuführen. So war z.B. eine Erhöhung des Ausländerbestandes dann erlaubt, wenn sie für die wirtschaftliche Entwicklung eines Kantons von besonderer Bedeutung war. ${ }^{19}$

\subsubsection{GLOBALPLAFONIERUNG (AB 1970)}

Die betriebsweise Plafonierung des Bestandes an ausländischen Arbeitskräften in den 60er Jahren schränkte die Entwicklungsmöglichkeiten der Wirtschaft stark ein. Zudem vermochte diese Regelung infolge ihres begrenzten Geltungsbereiches ein weiteres, wenn auch verlangsamtes Wachstum des Ausländerbestandes nicht zu verhindern. Zwar war es gelungen, die Zahl der kontrollpflichtigen ausländischen Arbeitskräfte ab 1965 annähernd zu stabilisieren. Die Möglichkeit zur Umwandlung von Saisonnierbewilligungen, die Erteilung von Niederlassungsbewilligungen und der Familiennachzug führten jedoch dazu, dass sich die Zahl der ausländischen Wohnbevölkerung von Ende 1963 bis Ende 1969 um 216'000 auf 972'000 Personen erhöhte (siehe TAB. 2.1).

Unter dem Druck der zweiten Überfremdungsinitiative ${ }^{20}$ führte der Bundesrat im Frühjahr 1970 die Globalplafonierung ein, deren Zielsetzung im wesentlichen darin bestand, den Zugang ausländischer Erwerbstätiger zum schweizerischen $\mathrm{Ar}$ -

19 Diese Ausnahmebestimmung galt im Gegensatz zu früheren Ausnahmebestimmungen nicht mehr nur für 'hochbefähigte Arbeitskräfte' und entsprach damit einer verstärkten Berücksichtigung der Interessen der peripheren Regionen.

20 Seit Ende der 60er Jahre konnten die Schweizer Stimmbürger mehrmals Stellung zu sog. 'Überfremdungsinitiativen' nehmen, bei denen es im Kern um die Begrenzung des Ausländerbestandes ging. Diese wurden vom Schweizer Volk ausnahmslos abgelehnt, wenn auch mit z.T. sehr knapper Mehrheit. Anfang Dezember 1996 kam es mit der sog. Asylinitiative, einer von der Schweizerischen Volkspartei lancierten Initiative 'gegen die illegale Einwanderung', zur bislang letzten ausländerpolitischen Abstimmung. 
beitsmarkt insgesamt zu kontrollieren und zu begrenzen. ${ }^{21}$ Die Höchstzahl der Neuzulassungen wird seither entsprechend den zu erwartenden Abgängen (Auswanderung, Heirat mit Schweizer Bürgern, Einbürgerungen, Sterbefälle, etc.) so berechnet, dass eine Stabilisierung des Ausländeranteils erreicht werden kann. Basierend auf dieser Höchstzahl, erfolgt die Festsetzung kantonaler Kontingente über einen Verteilungsschlüssel. Da Niedergelassene und Grenzgänger nicht der Globalplafonierung unterstehen, werden jährliche Kontingente lediglich für Jahresaufenthalter, Saisonniers und Kurzaufenthalter festgelegt. ${ }^{22}$

Aufgrund des antizipierten Anstiegs der ausländischen Wohnbevölkerung wurde in der Bundesratsverordnung vom 9. Juli 1975 nicht mehr nur die Stabilisierung sondern die Verringerung der ausländischen Wohnbevölkerung als Zielsetzung formuliert. ${ }^{23}$ Die Rezession Mitte der 70er Jahre sowie die damit verbundenen Rückwanderungen zahlreicher ausländischer Arbeitskräfte und deren Familienangehörigen in ihre Heimatländer führten dazu, dass die Zielvorgabe einer Reduktion des Bestandes der ausländischen Wohnbevölkerung erreicht werden konnte. Mit der Bundesratsverordnung vom 20. Oktober $1976^{24}$ wurde im Grundsatzartikel erstmals als Zielsetzung die Schaffung und Erhaltung eines ausgewogenen Verhältnisses zwischen dem Bestand der schweizerischen und ausländischen Bevölkerung formuliert, die auch heute noch Gültigkeit besitzt. ${ }^{25}$

Ungeachtet des massiven Anstiegs des Ausländeranteils seit dem Ende des Zweiten Weltkriegs findet sich für die Rezession Mitte der 70er Jahre empirische Evidenz für die Validität der oben erwähnten Konjunkturpufferfunktion ausländischer Arbeitskräfte. Gemäss den Berechnungen von ScHWARZ (1988) hat die Rotation der ausländischen Arbeitskräfte seit 1970 zwar abgenommen, es besteht aber dennoch bis heute eine beträchtliche Dynamik (siehe TAB. 2.5). Der durch die Rezession induzierte Beschäftigungsrückgang, der sich in der Periode von 1974 bis 1976 auf 7.6 Prozent belief und damit weit stärker ausfiel als in anderen westeuropäischen Staaten, wurde mehrheitlich von ausländischen Arbeitskräften getragen. ${ }^{26}$

21 Bundesratsbeschluss über die Begrenzung der Zahl der erwerbstätigen Ausländer vom 16. März 1970 [AS (1970: 305ff.)].

22 Kontingente für Kurzaufenthalter existieren erst seit 1974.

23 AS (1975: 1396ff.).

24 AS (1976: 2165ff.).

25 Verordnung des Bundesrates über die Begrenzung der Zahl der Ausländer [BVO (1986, Art. 1)].

26 SCHWARZ (1988). Der Beschäftigungsrückgang bei den ausländischen Arbeitskräften war rund sechsmal grösser als bei den Schweizern. Noch ausgeprägter stellt sich die Diskrepanz zwischen 1973 und 1977 dar, mit einen Rückgang der einheimischen Beschäftigung von le- 
Tab. 2.5: Ein- und Auswanderung erwerbstätiger Ausländer, 1970-1997

\begin{tabular}{|c|c|c|c|c|c|c|c|c|c|c|c|}
\hline Jahr & \multicolumn{4}{|c|}{ Rotationskoeffizient in Prozent } & $\begin{array}{l}\text { Migr.- } \\
\text { koeff. }\end{array}$ & Jahr & \multicolumn{4}{|c|}{ Rotationskoeffizient in Prozent } & $\begin{array}{l}\text { Migr.- } \\
\text { koeff. }\end{array}$ \\
\hline 1970 & 11.7 & 13.2 & 24.9 & -1.5 & 0.89 & 1984 & 4.7 & 5.4 & 10.0 & -0.7 & 0.87 \\
\hline 1971 & 8.8 & 9.3 & 18.1 & -0.5 & 0.95 & 1985 & 4.7 & 5.3 & 9.9 & -0.6 & 0.89 \\
\hline 1972 & 9.5 & 7.4 & 16.9 & 2.0 & 1.28 & 1986 & 5.3 & 5.1 & 10.4 & 0.1 & 1.04 \\
\hline 1973 & 9.1 & 8.1 & 17.1 & 1.0 & 1.12 & 1987 & 5.8 & 5.2 & 11.0 & 0.6 & 1.12 \\
\hline 1974 & 5.3 & 7.7 & 13.0 & 2.5 & 0.69 & 1988 & 5.8 & 5.4 & 11.3 & 0.4 & 1.07 \\
\hline 1975 & 3.1 & 11.5 & 14.7 & -8.4 & 0.27 & 1989 & 6.0 & 5.5 & 11.5 & 0.5 & 1.09 \\
\hline 1976 & 2.9 & 10.8 & 13.7 & -7.9 & 0.27 & 1990 & 7.1 & 5.5 & 12.7 & 1.7 & 1.29 \\
\hline 1977 & 3.9 & 8.1 & 12.0 & -4.2 & 0.48 & 1991 & 6.7 & 5.8 & 12.5 & 1.0 & 1.16 \\
\hline 1978 & 4.4 & 6.1 & 10.6 & -1.7 & 0.72 & 1992 & 5.6 & 6.7 & 12.3 & -1.1 & 0.84 \\
\hline 1979 & 5.2 & 5.7 & 10.9 & -0.5 & 0.91 & 1993 & 4.4 & 5.5 & 9.8 & -1.1 & 0.80 \\
\hline 1980 & 6.5 & 6.6 & 13.1 & -0.1 & 0.98 & 1994 & 3.9 & 4.5 & 8.4 & -0.6 & 0.87 \\
\hline 1981 & 6.9 & 6.5 & 13.5 & 0.4 & 1.06 & 1995 & 3.7 & 4.4 & 8.0 & -0.7 & 0.84 \\
\hline 1982 & 6.3 & 6.3 & 12.6 & 0.1 & 1.00 & 1996 & 3.4 & 4.5 & 7.9 & -1.1 & 0.75 \\
\hline 1983 & 4.5 & 6.0 & 10.6 & -1.5 & 0.75 & 1997 & 3.3 & 4.3 & 7.6 & -1.1 & 0.75 \\
\hline
\end{tabular}

Quelle: BFA (1998), eigene Berechnungen

Anmerkungen: Ausländer mit einer Niederlassungs- oder Jahresaufenthaltsbewilligung

Einwanderung (IM): Einwanderung*100/0.5*(Anfangsbestand + Endbestand)

Auswanderung (EM): Auswanderung*100/0.5*(Anfangsbestand + Endbestand)

Bruttomigration (GM): (Einwanderung + Auswanderung)*100/0.5*(Anfangsbestand + Endbestand)

Nettomigration (NM): (Einwanderung - Auswanderung)*100/0.5*(Anfangsbestand + Endbestand)

Migrationskoeffizient: Einwanderung/Auswanderung

Die Rezession zu Beginn der 80er Jahre führte demgegenüber zu geringeren Beschäftigungseffekten. Obschon zwischen 1982 und 1984 wiederum ein dreijähriger Beschäftigungsrückgang stattfand, belief sich dieser lediglich auf 2.1 Prozent. Zudem wurde er in erster Linie von Schweizern getragen (-2.9 Prozent). Für die Ausländer resultierte demgegenüber ein Beschäftigungszuwachs von 0.8 Prozent. Dieses Ergebnis ist umso erstaunlicher, als die ausländischen Arbeitskräfte im Mittel nicht nur weniger qualifiziert waren, sondern gleichzeitig auch eine unvorteilhaftere Branchenverteilung aufwiesen. ${ }^{27}$ Diese Ergebnisse müssen allerdings relativiert werden, wenn die Arbeitslosenzahlen mitberücksichtigt werden. Wie aus TAB. 3.6 hervorgeht, liegt die Arbeitslosenquote der Ausländer während des gesamten Betrachtungszeitraums deutlich über jener der Schweizer.

diglich 1.1 Prozent im Vergleich zu einem Beschäftigungsrückgang bei den Ausländern von 23.1 Prozent.

27 SCHWARZ (1988). 
Für diese divergierende Entwicklung in den Erwerbstätigen- und Arbeitslosenzahlen sowie für die Tatsache, dass die ausländischen Arbeitskräfte in der Rezession zu Beginn der 80er Jahre nicht mehr als Konjunkturpuffer fungierten, lassen sich verschiedene Gründe anführen. ${ }^{28}$ Erstens hatte sich die Zusammensetzung der ausländischen Bevölkerung im Zeitablauf stark verändert: der Anteil der Ausländer mit einer Niederlassungsbewilligung hatte sich seit 1973 von rund 31 Prozent auf nahezu 52 Prozent im Jahre 1981 erhöht. Bis 1995 ist der Anteil weiter auf rund 59 Prozent angestiegen. Da sich der Anteil der Ausländer mit einer Jahresaufenthaltsbewilligung zum heutigen Zeitpunkt auf rund 20 Prozent beläuft, ist der arbeitsmarktbezogene Handlungsspielraum der schweizerischen Ausländerpolitik auf lediglich rund 20 Prozent der Erwerbstätigen zusammengeschrumpft. Zweitens hatte sich die Zahl der ausländischen Erwerbstätigen zwischen den Vorrezessionsjahren 1973 und 1981 aufgrund erheblicher Rückwanderungen um 148'000 Personen reduziert, was einer Verringerung des Ausländeranteils um 4.1 Prozentpunkte entsprach.

Drittens hatte die Einführung der obligatorischen Arbeitslosenversicherung am 1. April 1977 als Reaktion auf die Rezession Mitte der 70er Jahre dazu geführt, dass ein Grossteil der ausländischen Arbeitnehmer in der Zwischenzeit Ansprüche auf Leistungen der schweizerischen Sozialkassen erworben hatte. ${ }^{29}$ Während der Rezession der 80er Jahre entfiel daher für viele ausländische Arbeitskräfte die Notwendigkeit einer Rückkehr in ihre Heimatländer. Verstärkt wurde dieser Effekt durch das geltende Ausländerrecht, das den Saisonniers das Recht auf eine längerfristige Aufenthaltsgenehmigung einräumte, wenn sie während vier aufeinanderfolgender Jahre jeweils 9 Monate in der Schweiz erwerbstätig gewesen waren. ${ }^{30}$ Da sie dieses Recht im Falle einer Rückwanderung verloren hätten, bestanden gute Gründe, auch im Falle einer Periode der Erwerbslosigkeit in der Schweiz zu verbleiben.

Der wirtschaftliche Aufschwung zu Beginn der 80er Jahre sowie der damit verbundene Arbeitskräftemangel führten zu einem massiven Wiederanstieg der ausländischen Wohnbevölkerung. Als die Ausländerbeschäftigung im Jahre 1991 ihren vorläufigen Höchststand erreichte, waren rund 1.05 Mio. Ausländer in der Schweiz erwerbstätig, was einem Anteil an der Gesamtbeschäftigung von rund 27

28 FISCHER/STRAUBHAAR (1996b).

29 Bis März 1977 beruhte der Versicherungsschutz im Falle von Arbeitslosigkeit teilweise auf kantonalen Obligatorien sowie teilweise auf Freiwilligkeit und erfasste daher nur einen geringen Teil der Arbeitnehmer. Zu Beginn der Rezession Mitte der 70er Jahre waren lediglich rund 20 Prozent der Arbeitnehmer gegen Arbeitslosigkeit und witterungs- bzw. wirtschaftlich bedingte Verdienstausfälle versichert [siehe SHELDON (1993)].

Dieser Aspekt ist deshalb von Bedeutung, weil gemäss den Berechnungen von DHIMA (1991) rund zwei Drittel der längerfristig in der Schweiz lebenden Ausländer als Saisonniers in die Schweiz eingereist waren. 
Prozent entsprach. ${ }^{31}$ Auf den Aufschwung der 80er Jahre folgte aber nicht nur ein massiver Anstieg der Zahl der ausländischen Erwerbstätigen, sondern auch eine markante Veränderung in der Zusammensetzung der Zuwanderung. Erstens verringerte sich die Attraktivität des schweizerischen Arbeitsmarktes, weil sich infolge der Eingliederung der südeuropäischen Staaten in die EU die wirtschaftlichen Rahmenbedingungen geändert hatten. Diese Entwicklung hatte verstärkte Rückwanderungsbewegungen in die traditionellen Herkunftsländer zur Folge, so dass sich der Anteil der Ausländer aus den Anrainerstaaten über die Jahre hinweg kontinuierlich reduziert hat und eine zunehmende Verlagerung der Zuwanderung ausländischer Arbeitskräfte an die Ränder des Mittelmeerraumes sowie nach Portugal und in den aussereuropäischen Raum stattgefunden hat.

Zweitens haben sich die Migrationsmotive im Zeitverlauf stark verändert. Bereits seit den 70er Jahren findet ein substantieller Teil der Zuwanderung im Rahmen der Familienmigration statt. In den 80er Jahren hat zudem die Flüchtlingsmigration verstärkt an Bedeutung gewonnen. ${ }^{32} \mathrm{Wie}$ aus TAB. 3.2 hervorgeht, hat die Arbeitsmigration im Zeitverlauf massiv an Bedeutung eingebüsst. Während sich der Anteil der Arbeitsmigration 1960 noch auf rund 85 Prozent belief, reduzierte er sich bis 1995 auf 35 Prozent. Diese Entwicklung hat mit zur Neuorientierung der schweizerischen Ausländerpolitik zu Beginn der 90er Jahre beigetragen.

Von zentraler Bedeutung für die geltende schweizerische Ausländerpolitik ist die Bundesratsverordnung vom 6. Oktober 1986 zur Begrenzung der Zahl der Ausländer (BVO). Sie beinhaltet einerseits die zentralen Zielsetzungen der schweizerischen Ausländerpolitik. Andererseits begründet sie ein Kontingentierungssystem, das die Zulassung ausländischer Arbeitskräfte zahlenmässig begrenzt. In der Bundesratsverordnung werden drei zentrale Ziele der schweizerischen Ausländerpolitik genannt (siehe TAB. 2.6). Daneben lassen sich gemäss DHIMA (1991) auch drei implizite Ziele anführen. Diese Zielsetzungen werden über quantitative und qualitative Anforderungen an die Zulassungspolitik verfolgt.

Die in der BVO enthaltenen Begrenzungsmassnahmen gelten grundsätzlich für alle Ausländer, sofern sie nicht im Besitz einer Niederlassungsbewilligung sind. Die Verordnung nennt auch die Voraussetzungen, unter denen die zuständigen Behörden eine Bewilligung erteilen oder verlängern können. Der Bundesrat setzt periodisch Höchstzahlen (Kontingente) für erstmals einreisende oder eine Erwerbstätigkeit aufnehmende Jahresaufenthalter, Saisonniers und Kurzaufenthalter fest. Diese Kontingente werden auf Bund und Kantone aufgeteilt. Für nicht-erwerbstätige Ausländer bestehen demgegenüber keine zahlenmässigen, dafür aber

31 HAUG (1995).

32 GROSSEN (1996) kommt in diesem Zusammenhang zu dem Schluss, dass die eigentliche Arbeitsmigration in verstärktem Masse mit der Asylmigration konkurriert. 
qualitative Begrenzungskriterien. So wird etwa das Vorhandensein ausreichender finanzieller Mittel für die Dauer des Aufenthalts vorausgesetzt. ${ }^{33}$

\section{Tab. 2.6: Ziele der schweizerischen Ausländerpolitik}

\begin{tabular}{|c|c|}
\hline \multirow[t]{3}{*}{ Explizite Ziele } & $\begin{array}{l}\text { 1. Ausgewogenes Verhältnis zwischen dem Bestand der schweizerischen } \\
\text { und der ausländischen Wohnbevölkerung }\end{array}$ \\
\hline & $\begin{array}{l}\text { 2. Schaffung günstiger Rahmenbedingungen für die Eingliederung der in } \\
\text { der Schweiz wohnenden und arbeitenden Ausländer }\end{array}$ \\
\hline & $\begin{array}{l}\text { 3. Verbesserung der Arbeitsmarktstruktur und eine möglichst ausgegli- } \\
\text { chene Beschäftigung }\end{array}$ \\
\hline \multirow[t]{3}{*}{ Implizite Ziele } & 4. Schutz einheimischer Arbeitnehmer \\
\hline & 5. Schutz peripherer Regionen \\
\hline & 6. Bewahrung der bestehenden ökonomischen Strukturen \\
\hline
\end{tabular}

Quelle: Golder/Straubhaar (1998)

Unter dem Begriff 'ausgewogenes Verhältnis' wird i.a. die relative, d.h. prozentuale Stabilisierung des ausländischen Bevölkerungsanteils an der Gesamtbevölkerung verstanden. Um die Erreichung der in TAB. 2.6 genannten Zielsetzungen zu gewährleisten, knüpfen sich an die erstmalige Erteilung sowie die Verlängerung von Aufenthaltsbewilligungen an Erwerbstätige gewisse Bedingungen. ${ }^{34}$ Erstens ist, wie bereits erwähnt, die Zahl der neueinreisenden erwerbstätigen Ausländer zahlenmässig begrenzt. Für Jahresaufenthalter, Saisonniers und Kurzaufenthalter existieren Kontingente (siehe auch TAB. 3.3), um ein ausgewogenes Verhältnis zwischen der schweizerischen und der ausländischen Bevölkerung sicherzustellen.

Zweitens bestehen im Rahmen der Drei-Kreise-Politik (siehe Abschnitt 2.3) aufgrund von Art. 8 BVO drei Stufen der Einwanderungsberechtigung, die nach geographisch-politischen Räumen gegliedert sind. Drittens gilt der sog. 'Inländervorrang', d.h. der Vorrang inländischer Arbeitnehmer bei Neueinstellungen. Dieser Grundsatz impliziert, dass Bewilligungen zur erstmaligen Erwerbstätigkeit, zum Stellen-, Berufs- oder Kantonswechsel sowie zur Verlängerung des Aufenthalts nur dann erteilt werden dürfen, "... wenn der Arbeitgeber keine einheimische Ar-

33 Eine Reihe von Personenkategorien sind sowohl von den quantitativen als auch den qualitativen Begrenzungskriterien ausgenommen. Dies betrifft insbesondere Asylsuchende, anerkannte Flüchtlinge, ausländische Ehegatten von Schweizerinnen und Schweizern und deren Kinder, ehemalige Schweizer Bürgerinnen und Bürger sowie die Ehegatten und die unter 18jährigen Kinder von Jahresaufenthaltern im Rahmen der Bestimmungen über den Familiennachzug. Ebenfalls ausgenommen sind Saisonniers, deren Saisonnierbewilligungen in Jahresaufenthaltsbewilligungen umgewandelt werden.

Siehe RAESS-EICHENBERGER/RAESS-EICHENBERGER (1997). 
beitskraft findet, die gewillt und fähig ist, die Arbeit zu den orts- und berufsüblichen Lohn- und Arbeitsbedingungen zu leisten." 35

Die Einschränkungen bezüglich des Stellen-, Berufs- und Kantonswechsels sind vor allem durch strukturpolitische Zielsetzungen begründet, da die Abwanderung ausländischer Arbeitskräfte aus strukturschwachen Branchen oder Regionen verhindern werden soll. Diese restriktive Regelung wurde jedoch im Zusammenhang mit den Revitalisierungsbestrebungen zu Beginn der 90er Jahre gelockert. Den Mobilitätsbestimmungen kommt aus verschiedenen Gründen zentrale Bedeutung zu. Einerseits dienen sie der Steuerung der Allokation der ausländischen Arbeitskräfte, andererseits als Tatbestandsvoraussetzung für die Inanspruchnahme von Leistungen der Arbeitslosenversicherung. Nur wer vermittlungsfähig, d.h. mobil ist, hat einen Anspruch auf Arbeitslosenentschädigung.

Viertens ist als weitere Voraussetzung zur Bewilligung einer Erwerbstätigkeit der Grundsatz gleicher Arbeits- und Lohnbedingungen zu nennen. "Bewilligungen dürfen nur erteilt werden, wenn der Arbeitgeber dem Ausländer die selben ortsund betriebsüblichen Lohn- und Arbeitsbedingungen bietet wie den Schweizern und der Ausländer angemessen gegen die wirtschaftlichen Folgen von Krankheit gesichert ist". ${ }^{36}$ Mit dieser Bestimmung sollen ausländische Arbeitskräfte vor missbräuchlichen Arbeits- und Lohnbedingungen und inländische Arbeitnehmer vor Lohndumping geschützt werden. Fünftens muss die Sorgfaltspflicht des Arbeitgebers hinsichtlich des rechtmässigen Aufenthalts der ausländischen Arbeitnehmer erfüllt sein. Sechstens wird schliesslich eine den örtlichen Verhältnissen angemessene Unterkunft vorausgesetzt. Abschliessend ist darauf hinzuweisen, dass die Bewilligung selbständiger Erwerbstätigkeit in der Schweiz restriktiv gehandhabt und nur in Ausnahmefällen gestattet wird. Lediglich Ausländer mit einer Niederlassungsbewilligung haben einen Anspruch auf Ausübung einer selbständigen Erwerbstätigkeit. Diese Bestimmung dient in erster Linie dem Schutz des einheimischen Gewerbes.

Nach rund einem Jahrzehnt des Aufschwungs setzte ab 1991 eine bis ins Jahr 1997 andauernde Rezessions- bzw. Stagnationsphase ein. Diese führte in der Folge zu einer Verringerung der Beschäftigung zwischen dem 2. Quartal 1991 und dem 4. Quartal 1997 von 3.921 Mio. auf 3.807 Mio. Erwerbstätige, was einem Beschäftigungsrückgang von $114^{\prime} 000$ Personen entspricht. ${ }^{37}$ Eine genauere Analyse dieser Entwicklung führt zu zwei interessanten Ergebnissen. Erstens hat sich

35 BVO (1986: Art. 7, Abs. 1). Als 'einheimische Arbeitskräfte' gelten Schweizer, Ausländer mit einer Niederlassungsbewilligung, anerkannte Flüchtlinge, ausländische Ehegatten von Schweizern sowie deren ausländische Kinder, ehemalige Schweizer Bürger, Liechtensteinische Landesbürger, Stagiaires sowie ausländische Jugendliche.

BVO (1986: Art. 9, Abs. 1).

37

BFS (1998a). 
der Konjunktureinbruch der 90er Jahre kaum auf die Zahl der erwerbstätigen Frauen ausgewirkt. Während sich der Rückgang bei den Männern auf 6.7 Prozent belief, hat bei den Frauen über diesen Zeitraum sogar ein Beschäftigungszuwachs von 3 Prozent stattgefunden. ${ }^{38}$ Zweitens hat dieser Beschäftigungsrückgang vor allem zu Lasten der Ausländer stattgefunden. Zwischen dem 2. Quartal 1991 und dem 4. Quartal 1997 hat sich die Zahl der ausländischen Erwerbstätigen um 145'000 Personen verringert (Männer: 143'000 Personen, Frauen: 2'000 Personen). Im gleichen Zeitraum hat die Beschäftigung der Inländer um 30'000 Personen zugenommen, wobei dies ausschliesslich auf einen Beschäftigungszuwachs bei den Frauen von $48^{\prime} 000$ Personen zurückzuführen ist. ${ }^{39}$

Dieser markante Beschäftigungsrückgang bei den Ausländern hat vor allem auf Kosten der Saisonniers, Grenzgänger und Asylbewerber stattgefunden. So ist die Zahl der Saisonniers von 108'000 Personen im 2. Quartal 1991 auf 28'000 Personen im 2. Quartal 1997, die Zahl der Grenzgänger von 183'000 auf 143'000 Personen sowie die Zahl der erwerbstätigen Asylbewerber von 34'000 auf 18'000 Personen gefallen. ${ }^{40}$ Bei den Niedergelassenen und Jahresaufenthaltern hat demgegenüber kein Beschäftigungsrückgang, sondern ein leichter Zuwachs stattgefunden. ${ }^{41}$ Die gegenläufige Entwicklung, nämlich des Abbaus von Arbeitsplätzen bei gleichzeitiger Zunahme der erwerbsfähigen Bevölkerung aufgrund der demographischen Entwicklung, war mit für den massiven Anstieg der Arbeitslosenzahlen verantwortlich. Dieser belief sich im Zeitraum von 1990 bis 1997 auf 1038 Prozent. ${ }^{42}$ Diese Entwicklung wurde vor allem von den schlecht qualifizierten Arbeitnehmern sowie den Beschäftigten in strukturschwachen Branchen, wie der Textil-, Maschinen-, und Metallindustrie getragen, die vom internationalen Wettbewerbsdruck und Strukturwandel besonders stark betroffen waren. Es ist daher nicht erstaunlich, dass die ausländischen Arbeitnehmer 1997 mit rund 10.7 Prozent eine rund dreimal so hohe Arbeitslosenquote aufwiesen wie die schweizerischen Arbeitnehmer (3.6 Prozent). Im Jahresdurchschnitt 1997 waren rund 46 Prozent der eingeschriebenen Arbeitslosen Ausländer. ${ }^{43}$

38 Dieses Ergebnis impliziert allerdings nicht, dass die Frauen von der Konjunkturschwäche profitiert haben, denn es ist zu vermuten, dass viele Frauen aufgrund des Stellenverlustes ihrer Partner gezwungen waren, eine Erwerbstätigkeit aufzunehmen.

39 BFS (1998a).

40 BFS (1998a).

41 HaUg (1995).

42 Die Zahl der Arbeitslosen lag 1990 bei 18'133 Personen und erhöhte sich bis 1996 auf 188'304 Personen [BFS (1998b)].

BFS (1998b). 


\subsection{DREI-KREISE-POLITIK}

Die erfolglosen Bemühungen zur Stabilisierung des Ausländeranteils, die verstärkte Substitution der traditionellen durch neue Herkunftsregionen sowie die Veränderungen der Migrationsmotive seit Beginn der 70er Jahre haben den Bundesrat zu Beginn der 90er Jahre veranlasst, mit der 'Fremdarbeiterpolitik' der Nachkriegsjahre zu brechen und eine Neuorientierung der schweizerischen Ausländerpolitik zu vollziehen. Der Reformbedarf lässt sich aber auch durch einige weitere Argumente verdeutlichen.

Erstens trugen die bestehenden Regelungen den qualitativen Anforderungen des schweizerischen Arbeitsmarktes zu wenig Rechnung, was sich in einem Missverhältnis zwischen Arbeitsangebot und -nachfrage niederschlug. Insbesondere im Rahmen des Saisonnierstatuts sind vorwiegend schlecht qualifizierte ausländische Arbeitskräfte eingewandert, die zu einer Aufblähung des industriellen Sektors beigetragen haben. Damit wurde der Aufbau eines leistungsfähigen Dienstleistungssektors behindert und der Strukturwandel verlangsamt. Diese Fehlentwicklung spiegelt sich im überproportional hohen Anteil erwerbsloser Ausländer wider.

Zweitens sehen sich die westeuropäischen Industriestaaten, wie bereits einleitend erwähnt, einem wachsenden Migrationspotential gegenüber, nicht nur aufgrund der Probleme auf dem Balkan, sondern auch wegen der Öffnung der Staaten Mittel- und Osteuropas und der demographischen und politischen Entwicklung im Nahen Osten und in Nordafrika. Diese haben bereits in den 80er Jahren einen markanten Anstieg der Zahl der Asylbewerber zur Folge gehabt und damit die erwähnten strukturellen Veränderungen der Zuwanderung mitbeeinflusst.

Drittens haben die Vollendung des europäischen Binnenmarktes Anfang 1993 sowie das schweizerische Nein zum EWR Ende 1992 dazu geführt, dass die Schweiz im europäischen Integrationsprozess eine Aussenseiterrolle übernommen hat. Die Schweizer Wirtschaft ist aus diesem Grund nicht nur im Wettbewerb um Absatzmärkte gegenüber den europäischen Mitkonkurrenten benachteiligt, sondern "... verliert gerade für jene Personen an Anziehungskraft, die sie aufgrund ihrer Qualifikationen und ihrer sprachlichen und kulturellen Nähe wieder vermehrt gewinnen möchte." 44

Vor diesem Hintergrund ist die jüngste ausländerpolitische Diskussion zu sehen, die im Januar 1989 mit dem 'Strategiebericht für eine Flüchtlings- und Asylpolitik der 90er Jahre' einer interdepartementalen Arbeitsgruppe einsetzte. Dieser Bericht befasste sich mit den Zusammenhängen zwischen der Ausländer-, der Asyl- und der internationalen Flüchtlingspolitik. Im nachfolgenden Bericht des BWA (früher BIGA) und BFA über die 'Konzeption und Prioritäten der schweizerischen Aus-

44 HaUg (1995). 
länderpolitik der neunziger Jahre' vom April 1991, wurde erstmals die sog. DreiKreise-Politik vorgestellt. Beide Berichte bildeten die Grundlage für den im Mai 1991 vom Bundesrat verabschiedeten 'Bericht zur Ausländer- und Flüchtlingspolitik', mit dem die erwähnte Abkehr von der 'Fremdarbeiterpolitik' der Nachkriegszeit vollzogen werden sollte.

Die Neukonzeption der schweizerischen Ausländerpolitik suchte zwei wichtige Anliegen zu berücksichtigen. Zum einen handelte es sich um die angestrebte Beteiligung der Schweiz am europäischen Integrationsprozess und zum anderen ging es um die Berücksichtigung des sich weltweit verschärfenden Migrationsproblems. Im Zentrum dieser Neukonzeption stand die Drei-Kreise-Politik. Im Gegensatz zur bisherigen Rekrutierungspolitik wurden, nach geographisch-politischen Räumen gegliedert, drei Stufen der Einwanderungsberechtigung definiert, die sowohl den arbeitsmarkt- als auch den integrationspolitischen Bedürfnissen der schweizerischen Volkswirtschaft Rechnung zu tragen suchten. Die drei Kreise lassen sich wie folgt charakterisieren: 45

\section{Tab. 2.7: Drei-Kreise-Politik}

a. In einem innersten Kreis, beschränkt auf die Staaten der EG und der EFTA, wird der Personenverkehr schrittweise von den bestehenden ausländerpolitischen und arbeitsmarktlichen Beschränkungen befreit. Die umfassendste Form der Liberalisierung wäre die Realisierung des freien Personenverkehrs im Sinne des Rechts der EG. ...

b. Zu einem mittleren Kreis gehören die Länder, die weder der EG noch der EFTA angehören, also nicht Teil des innersten Kreises sein können, aber dennoch die Funktion traditioneller Rekrutierungsgebiete übernehmen sollen. Dazu werden insbesondere die USA und Kanada zu zählen sein, eventuell - im Blick auf die heutige Praxis - auch Jugoslawien, Australien und Neuseeland. Im Vergleich zur hergebrachten Ausländerpolitik sollen Spitzenkräfte aus diesen Ländern erleichtert zugelassen werden. Administrative Vereinfachungen, Verbesserungen in der Rechtsstellung, Unterstützung der beruflichen Weiterbildung und der Integration sind möglich. Grundsätzlich soll aber die bisherige Begrenzungspolitik weitergeführt werden.

c. Im äussersten Kreis befinden sich alle übrigen Staaten. Ihnen gegenüber ist an der bisherigen Ausländerpolitik festzuhalten. Angehörige dieser Staaten sollen weiterhin eine Aufenthalts- und Arbeitsbewilligung in der Schweiz nur in Ausnahmefällen erhalten. ... Lockerungen dieser strengen Praxis sind allenfalls gegenüber hochqualifizierten Spezialisten für einen mehrjährigen, aber zeitlich begrenzten Aufenthalt möglich.

Quelle: BIGABBFA (1991)

45 Gemäss dem Bundesratsbeschluss vom 23. September 1991 zählt Jugoslawien seit dem 1. November 1991 nicht mehr zu den traditionellen Rekrutierungsländern. Dieser Beschluss gilt für alle Angehörigen dieses Landes, die noch nie oder nicht während der letzten Jahre in der Schweiz gearbeitet haben, und umfasst alle Bewilligungskategorien. 
Neu an dieser Drei-Kreise-Politik war vor allem die angestrebte vollständige Freizügigkeit für Angehörige des innersten Kreises sowie der faktische Einwanderungsstopp für ausländische Arbeitskräfte aus nicht-europäischen Staaten. Während letzterer bereits implementiert worden ist, ist die Zielsetzung der Freizügigkeit nach wie vor Gegenstand der bilateralen Verhandlungen zwischen der Schweiz und der EU.

\subsection{ZWEI-KREISE-POLITIK ?}

Basierend auf der Motion Simmen wurde der Bundesrat im März 1993 vom National- und Ständerat beauftragt, ein Migrationsgesetz auszuarbeiten. Es folgte der 'Bericht über eine schweizerische Migrationspolitik' vom März 1995, der im Auftrag des Bundesrates verfasst wurde und darauf abzielte, die Probleme der bestehenden Politik zu analysieren und einige grundsätzliche Optionen einer künftigen Migrationspolitik zu skizzieren. Die Vernehmlassung zum Bericht zeigte indes, dass zwar die Mehrheit der Parteien, Verbände und Organisationen eine umfassende Migrationspolitik befürwortete, dass aber sehr unterschiedliche Auffassungen hinsichtlich der effektiven Ausgestaltung der Migrationspolitik bestanden. ${ }^{46}$ Während zwar anerkannt wurde, dass zwischen der Ausländer-, der Asyl- und der internationalen Flüchtlingspolitik zahlreiche Überschneidungen und Kooperationsmöglichkeiten bestehen, sprach sich die Mehrheit doch deutlich für eine Trennung der Asyl- und Ausländerpolitik aus.

Mit Blick auf die Ergebnisse des Vernehmlassungsverfahrens setzte der Bundesrat im September 1996 eine Expertenkommission ein, die Vorschläge für eine künftige Migrationspolitik erarbeiten sollte. Der Bericht der Expertenkommission 'Ein neues Konzept der Migrationspolitik' vom August 1997 nennt als Zielsetzung die Schaffung einer kohärenten und transparenten Gesamtpolitik, welche die Ausländer- und Flüchtlingspolitik aufeinander bezieht, ohne allerdings die beiden unterschiedlich motivierten Politiken zu verschmelzen. Handlungsbedarf sieht die Kommission vor allem in der Neugestaltung und Vereinfachung des Zulassungsverfahrens durch Schaffung einer klaren rechtlichen Grundlage, verstärkten Integrationsbemühungen, dem Entgegenwirken illegaler Aufenthalte im Rahmen der Ausreisepolitik sowie der Systematisierung der Migrationsaussenpolitik.

Wie bereits erwähnt, ist die traditionelle Fremdarbeiterpolitik der Schweiz von regionalen und branchenspezifischen Partikularinteressen dominiert, fördert die Zuwanderung billiger Arbeitskräfte und hemmt daher unvermeidliche Strukturbereinigungen. Vor diesem Hintergrund schlägt die Kommission als Alternative ein Zulassungsmodell vor, das sich am Bedarf an gut- und hochqualifizierten Arbeitskräften orientiert. Aufgrund der Tatsache, dass die 'Qualität' der ausländischen Arbeitskräfte im Rahmen der Drei-Kreise-Politik nicht direkt berücksichtigt wer-

46 EXPERTENKOMMISSION MIGRATION (1997). 
den kann, plädiert die Expertenkommission für ein neues Zulassungssystem, eine sog. Zwei-Kreise-Politik, die lediglich noch zwischen den EWR-Staaten, dem vormaligen ersten Kreis, und allen anderen Herkunftsländern, d.h. dem vormaligen zweiten und dritten Kreis, unterscheidet.

Die Zulassung von EWR-Ausländern soll weiterhin den Grundsätzen der Personenfreizügigkeit folgen, wie sie in den bilateralen Verhandlungen mit der EU angestrebt wird. Die Zulassung von Arbeitskräften von ausserhalb des EWR-Raums, dem zweiten Kreis des neuen Systems, soll gemäss den Vorschlägen der Kommission kontingentiert bleiben und nach qualitativen Gesichtspunkten im Rahmen eines sog. Punktesystems erfolgen. So ermöglicht diese Neuorientierung einerseits eine direkte Berücksichtigung der individuellen sozio-ökonomischen Profile der ausländischen Arbeitskräfte, andererseits würde mit diesem Übergang auch die diskriminierende Behandlung potentieller Einwanderer wegfallen, denn Zuwanderungsberechtigungen würden nicht mehr aufgrund der Staatsangehörigkeit, sondern aufgrund spezifischer sozio-ökonomischer Charakteristika der einzelnen Wirtschaftssubjekte erteilt. Die materielle Ausgestaltung würde sich, den erfolgreichen Modellen Kanadas und Australiens folgend, auf Auswahlkriterien wie das Alter, die Ausbildung, die Berufserfahrung, die Sprachkenntnisse, das Vorhandensein eines Arbeitsplatzes sowie die berufliche Integrationsfähigkeit abstützen.

Die Expertenkommission empfiehlt ferner eine Verringerung der Vielzahl verschiedener Aufenthaltsregelungen auf drei Aufenthaltskategorien (siehe TAB. 2.8). Der Dauerstatus impliziert eine automatische Bewilligungsverlängerung, die Möglichkeit beruflicher und geographischer Mobilität sowie die Niederlassungsbewilligung nach zehn Jahren oder, gemäss einzelnen Staatsverträgen, nach fünf Jahren. Das Saisonnierstatut wird ersetzt durch einen Kurzaufenthalterstatus, der Interessenten von ausserhalb des EWR-Raums einen befristeten Arbeitseinsatz von maximal zwei Jahren ermöglicht. Die Aufnahme von Flüchtlingen erfolgt weiterhin im Rahmen der völkerrechtlichen Verpflichtungen und der humanitären Tradition. Asylsuchende im Verfahren, Schutzbedürftige, vorläufig Aufgenommene und Ausländer, deren Ausreise blockiert ist, werden unter dem Provisorischen Status zusammengefasst.

Als wesentlichen Bestandteil des neuen Konzepts sieht die Expertenkommission eine aktive Integrationspolitik vor. So soll der Zugang zum Arbeitsmarkt erleichtert und die Flexibilität der Ausländer erhöht werden. Desweiteren sollen verstärkte Anstrengungen unternommen werden, um die Chancengleichheit hinsichtlich der Ausbildung und der beruflichen Zukunft ausländischer Kinder zu verbessern, unabhängig vom ausländerrechtlichen Status der Eltern. Im sozialen Bereich wird vorgeschlagen, den Familiennachzug stärker als Integrationsmassnahme zu betrachten. Schliesslich sollen Daueraufenthaltern sektoriell politische Rechte zugestanden und die Einbürgerungspraxis liberalisiert werden. 
Tab. 2.8: Aufenthaltsbewilligungen gemäss Zwei-Kreise-Politik

\begin{tabular}{|c|c|c|c|c|}
\hline Status & Dauer & Familiennachzug & $\begin{array}{l}\text { Arbeitsbe- } \\
\text { willigung }\end{array}$ & Mobilität \\
\hline Dauerstatus & $\begin{array}{l}E U \text { : Aufenthaltsregelung } \\
\text { gemäss bilateralem Ab- } \\
\text { kommen; Niederlassung } \\
\text { gemäss heutiger Praxis } \\
\text { nach fünf Jahren } \\
\text { Nicht-EU: Zulassung } \\
\text { gemäss Punktesystem; } \\
\text { Anspruch auf Niederlas- } \\
\text { sung nach zehn Jahren } \\
\text { bzw. bilateralen Ab- } \\
\text { kommen }\end{array}$ & $\begin{array}{l}\text { EU: sofort, wenn ange- } \\
\text { messene Wohnung vor- } \\
\text { handen } \\
\text { Nicht-EU: sofort, wenn } \\
\text { angemessene Wohnung, } \\
\text { Zusammenleben und } \\
\text { ausreichende finanzielle } \\
\text { Mittel gewährleistet sind }\end{array}$ & $\begin{array}{l}\text { Nicht-EU: } \\
\text { ja }\end{array}$ & $\begin{array}{l}\text { Nicht-EU: } \\
\text { ja }\end{array}$ \\
\hline $\begin{array}{l}\text { Befristeter Status } \\
\text { Kurzaufenthalter- } \\
\text { status für Er- } \\
\text { werbstätige }\end{array}$ & $\begin{array}{l}E U: \text { Aufenthaltsstatus } \\
\text { gemäss bilateralem Ab- } \\
\text { kommen; befristet auf } \\
\text { maximal 1 Jahr } \\
\text { Nicht-EU: Zulassung } \\
\text { gemäss Punktesystem; } \\
\text { befristet auf maximal } 2 \\
\text { Jahre } \\
\text { je nach Aufenthalts- } \\
\text { zweck }\end{array}$ & $\begin{array}{l}E U: \text { sofort, wenn ange- } \\
\text { messene Wohnung vor- } \\
\text { handen } \\
\text { Nicht-EU: sofort, wenn } \\
\text { angemessene Wohnung, } \\
\text { Zusammenleben und } \\
\text { ausreichende finanzielle } \\
\text { Mittel gewährleistet sind } \\
\text { EU: ja } \\
\text { Nicht-EU: Nur in Aus- } \\
\text { nahmefällen }\end{array}$ & $\begin{array}{l}E U: \text { ja } \\
\text { Nicht-EU: } \\
\text { ja }\end{array}$ & $\begin{array}{l}E U: \mathrm{ja} \\
\text { Nicht-EU: } \\
\text { ja } \\
\\
\text { EU: ja } \\
\text { Nicht-EU: } \\
\text { nein }\end{array}$ \\
\hline $\begin{array}{l}\text { Provisorischer } \\
\text { Status } \\
\text { Asylsuchende im } \\
\text { Verfahren, vor- } \\
\text { läufig Aufge- } \\
\text { nommene, } \\
\text { Schutzbedürftige } \\
\text { und Ausländer, } \\
\text { deren Vollzug } \\
\text { blockiert ist }\end{array}$ & $\begin{array}{l}\text { Asylsuchende im Verfah- } \\
\text { ren, vorläufig Aufge- } \\
\text { nommene: offen bis zur } \\
\text { definitiven Entscheidung } \\
\text { resp. effektiven Ausreise } \\
\text { Schutzbedürftige: befri- } \\
\text { stet bis Ende des } \\
\text { Schutzbedürfnisses }\end{array}$ & $\begin{array}{l}\text { Asylsuchende und vor- } \\
\text { läufig Aufgenommene: } \\
\text { nein }\end{array}$ & $\begin{array}{l}\text { Alle: ja, } \\
\text { nach drei } \\
\text { bzw. sechs } \\
\text { Monaten }\end{array}$ & Alle: nein \\
\hline
\end{tabular}

Quelle: Expertenkommission Migration (1997)

Die Vorschläge dieser Studie der Expertenkommission werden im wesentlichen durch eine gleichzeitig verfasste Studie bestätigt, die auf der Grundlage der Analyse der Arbeitsmarktperformance der Einwanderer zu weitgehend identischen Schlussfolgerungen hinsichtlich der Neuorientierung der schweizerischen Migrationspolitik kommt [GoLDER (1997b)]. Diese Zusammenhänge werden im Schlusskapitel dieser Studie ausführlich diskutiert. 


\subsection{FAZIT}

Aus den Ausführungen dieses Kapitel ergeben sich zwei wesentliche Schlussfolgerungen, die für die Evaluation der Arbeitsmarktperformance der Einwanderer von Bedeutung sind. Erstens haben massive Migrationsbewegungen, insbesondere seit dem Ende des Zweiten Weltkriegs, dazu geführt, dass die Schweiz im Vergleich zu den anderen europäischen Staaten einen markant höheren Ausländeranteil aufweist, der sich gegenwärtig auf rund 19 Prozent der Gesamtbevölkerung beläuft.

Zweitens hat im Zeitverlauf eine Veränderung der Zuwanderungsstruktur stattgefunden. Einerseits haben sich während der vergangenen drei Jahrzehnte die $\mathrm{Zu}$ wanderungsströme von den Anrainerstaaten der Schweiz an den Rand des Mittelmeerraums sowie in den aussereuropäischen Raum verlagert. Diese Entwicklung ist auf den wirtschaftlichen Aufschwung in den traditionellen Rekrutierungsgebieten und die damit verbundene Verbesserung der Arbeitsmarktlage zurückzuführen, die, wie im Falle Italiens, zu massiven Rückwanderungsbewegungen geführt hat. Gleichzeitig haben sich die Migrationsmotive verändert. Während in den 50er und 60er Jahren die Arbeitsmigration dominierte, hat, spätestens seit den 70er Jahren, die Zuwanderung im Rahmen des Familiennachzugs und, insbesondere seit den 80er Jahren, die Flüchtlingsmigration an Bedeutung gewonnen. Die Zunahme der Familienmigration ist darauf zurückzuführen, dass sich die anfänglich temporär eingewanderten ausländischen Arbeitskräfte mehrheitlich für einen permanenten Aufenthalt in der Schweiz entschieden haben und daher ihre Familien nachkommen liessen. Die verstärkte Flüchtlingsmigration ist mit der $\mathrm{Zu}-$ nahme politischer Wirren und ethnischer Konflikte in vielen Regionen dieser Erde verbunden.

Diese Veränderungen in der Struktur und Zusammensetzung der Zuwanderung waren im wesentlichen für die Neuorientierung der schweizerischen Migrationspolitik zu Beginn der 90er Jahre verantwortlich. Die Zielsetzungen im Rahmen der Drei-Kreise-Politik bestanden einerseits darin, die Schweiz verstärkt in den europäischen Integrationsprozess einzubinden. Durch die Definition von drei Stufen der Einwanderungsberechtigung nach geographisch-politischen Räumen sollte andererseits auch verstärkt arbeitsmarkt- und integrationspolitischen Zielsetzungen Rechnung getragen werden. Die wachsende Kritik an der Drei-KreisePolitik, die sich erstens auf die ungenügende Koordination der Ausländer-, Asylund internationalen Flüchtlingspolitik, zweitens auf die diskriminierende Behandlung potentieller Einwanderer sowie drittens auf die unzureichende Berücksichtigung der individuellen Charakteristika der Zuwanderer richtete, hat dazu geführt, dass heute der Übergang zu einer Zwei-Kreise-Politik gefordert wird, welche diese Aspekte berücksichtigen würde. 


\section{DESKRIPTIVE ANALYSE DER ARBEITSMARKTPERFORMANCE}

Nachdem in Kapitel 2 die historische Entwicklung der Zuwanderung sowie die damit verbundenen Veränderungen der Migrationspolitik dargestellt wurden, geht es nun darum, ein stilisiertes statistisches Portrait der Ausländer zu zeichnen, um erste Hinweise auf die sozio-demographischen Profile und damit verbunden die Arbeitsmarktperformance der Einwanderer zu gewinnen.

\subsection{AUSLÄNDERANTEIL IN DER SCHWEIZ UND IM INTERNATIONALEN VERGLEICH}

Der historische Überblick über die Migrationsbewegungen in die Schweiz hat gezeigt, dass der Ausländeranteil in diesem Jahrhundert relativ grossen Schwankungen unterworfen war (siehe TAB. 2.1). Auf Phasen starker Zuwanderung, die in der Regel die Folge raschen Wirtschafts- und Arbeitsplatzwachstums waren, folgten Phasen der Emigration bzw. Rückwanderung in Zeiten des Krieges oder wirtschaftlichen Abschwungs.

Seit dem Ende des Zweiten Weltkriegs hat jedoch eine massive Zunahme des Ausländerbestandes stattgefunden. Dieser Umstand wird in TAB. 3.1 deutlich, aus der hervorgeht, dass die Schweiz nach Luxemburg und Australien den höchsten Ausländeranteil aller OECD-Staaten besitzt. ${ }^{47}$ Der Ausländeranteil der meisten europäischen OECD-Staaten liegt auf einem deutlich tieferen Niveau. Bemerkenswert ist in diesem Zusammenhang die Tatsache, dass ausser in der Schweiz lediglich in Belgien und Luxemburg die Mehrheit der Ausländer aus dem EUbzw. EWR-Raum stammt. ${ }^{48}$ In den anderen europäischen Staaten stammen die grössten ausländischen Bevölkerungsgruppen aus der Türkei, Ex-Jugoslawien, Marokko und Algerien.

Werden anstelle der Ausländeranteile die Anteile der im Ausland Geborenen betrachtet, so ergibt sich eine gewisse Konvergenz in den Anteilen zwischen den verschiedenen Staaten. Der Vorteil dieser Definition besteht darin, dass sich dadurch Verzerrungen, die auf unterschiedlich restriktive Einbürgerungspolitiken zurückzuführen sind, vermeiden lassen. Im Vergleich zu Ländern wie den Verei-

47 HAUG (1995) bemerkt in diesem Zusammenhang jedoch, dass Luxemburg sowohl aufgrund seiner Grösse als auch der Bedeutung seines internationalen Dienstleistungssektors weniger mit der Schweiz als Ganzem, sondern viel eher mit dem Kanton Genf, der einen Ausländeranteil von $37 \%$ besitzt, verglichen werden sollte.

48 In der Schweiz ist allerdings der Anteil der Ausländer aus dem EU- bzw. EWR-Raum stark rückläufig (siehe TAB. 3.3). 
nigten Staaten, Kanada oder Australien, wird in der Schweiz eine sehr restriktive Einbürgerungspolitik praktiziert, weshalb in der Schweiz zwischen dem Anteil der im Ausland Geborenen und dem Ausländeranteil kein signifikanter Unterschied besteht.

Tab. 3.1: Ausländeranteil an der Bevölkerung und Beschäftigung in ausgewählten OECD Staaten, 1993

\begin{tabular}{|c|c|c|c|c|}
\hline Land & $\begin{array}{c}\text { Ausländische } \\
\text { Bevölkerung } \\
\text { (in 1'000) }\end{array}$ & $\begin{array}{c}\text { Erwerbstätige } \\
\text { Ausländer } \\
\text { (in 1'000) }\end{array}$ & $\begin{array}{c}\text { Ausländische } \\
\text { Bevölkerung } \\
\text { (in \%) }\end{array}$ & $\begin{array}{c}\text { Erwerbstätige } \\
\text { Ausländer } \\
\text { (in \%) }\end{array}$ \\
\hline Belgien & 921 & 340 & 9.1 & 8.3 \\
\hline Dänemark & 189 & 54 & 3.6 & 1.9 \\
\hline Deutschland & $6 ' 878$ & $3^{\prime} 432$ & 8.5 & 8.8 \\
\hline Finnland & 56 & - & 1.1 & - \\
\hline Frankreich & 3'597 & $1 ' 544$ & 6.3 & 6.2 \\
\hline$U K$ & $2^{\prime} 001$ & 1'026 & 3.5 & 3.6 \\
\hline Irland & 94 & 40 & 2.7 & 3.0 \\
\hline Italien & 987 & - & 1.7 & - \\
\hline Japan & $1^{\prime} 321$ & - & 1.1 & - \\
\hline Luxemburg & 125 & 65 & 31.1 & 38.6 \\
\hline Niederlande & 780 & 278 & 5.1 & 3.9 \\
\hline Norwegen & 162 & 48 & 3.8 & 4.5 \\
\hline Österreich & 690 & 305 & 8.6 & 9.6 \\
\hline Schweden & 508 & 221 & 5.8 & 5.1 \\
\hline Spanien & 430 & 82 & 1.1 & 0.5 \\
\hline Schweiz & $1 ' 260$ & 726 & 18.1 & 21.7 \\
\hline Australien & $4^{\prime} 125$ & $2^{\prime} 164$ & 22.7 & 24.8 \\
\hline Kanada & $4^{\prime} 343$ & $2^{\prime} 681$ & 15.6 & 18.5 \\
\hline$U S A$ & $19^{\prime} 767$ & $11^{\prime} 636$ & 7.9 & 9.3 \\
\hline
\end{tabular}

Quelle: HAUG (1995), SOPEMI (1995)

Anmerkungen: Für Australien, Kanada und die USA beziehen sich die Zahlen auf das Jahr 1991 sowie auf im Ausland Geborene

\subsection{DEMOGRAPHISCHE MERKMALE DER AUSLÄNDISCHEN WOHNBEVÖLKERUNG}

Über die Zusammensetzung der Zuwanderung lässt sich Aufschluss gewinnen, indem zwischen Zuwanderung mit der Zielsetzung der Erwerbstätigkeit, d.h. Arbeitsmigration, und alternativer Zuwanderung, d.h. Familienmigration und Flüchtlingsmigration, unterschieden wird. Wie aus TAB. 3.2 hervorgeht, hat die 
Arbeitsmigration im Zeitverlauf kontinuierlich zugunsten der Familien- und Flüchtlingsmigration an Bedeutung verloren. ${ }^{49}$ Im Verlauf dieses Jahrhunderts, vor allem aber in den vergangenen $30 \mathrm{Jahren}$, hat eine fundamentale Verlagerung der Herkunftsländer der Zuwanderer von den Anrainerstaaten hin zu den peripheren europäischen bzw. aussereuropäischen Regionen stattgefunden.

Tab. 3.2: Zuwanderung in die Schweiz (in Prozent), 1960 - 1997

\begin{tabular}{|c|c|c|c|c|c|c|c|}
\hline Jahr & Erwerbstätig & $\begin{array}{c}\text { Nicht- } \\
\text { Erwerbstätig }\end{array}$ & Total & Jahr & Erwerbstätig & $\begin{array}{c}\text { Nicht- } \\
\text { Erwerbstätig }\end{array}$ & Total \\
\hline 1960 & 15.2 & 84.8 & $162 ' 428$ & 1979 & 51.9 & 48.1 & $60 ' 587$ \\
\hline 1961 & 14.0 & 86.0 & $204^{\prime} 862$ & 1980 & 50.9 & 49.1 & $75^{\prime} 262$ \\
\hline 1962 & 14.6 & 85.4 & $210^{\prime} 184$ & 1981 & 51.5 & 48.5 & $87 ' 823$ \\
\hline 1963 & 17.4 & 82.6 & $199^{\prime} 469$ & 1982 & 50.1 & 49.9 & $83^{\prime} 081$ \\
\hline 1964 & 18.7 & 81.3 & $196 ' 100$ & 1983 & 52.2 & 47.8 & $65^{\prime} 960$ \\
\hline 1965 & 23.3 & 76.7 & $141^{\prime} 375$ & 1984 & 50.5 & 49.5 & $66^{\prime} 961$ \\
\hline 1966 & 22.9 & 77.1 & $140^{\prime} 730$ & 1985 & 49.5 & 50.5 & $68^{\prime} 885$ \\
\hline 1967 & 21.5 & 78.5 & $127^{\prime} 981$ & 1986 & 49.1 & 50.9 & $76^{\prime} 432$ \\
\hline 1968 & 24.6 & 75.4 & $137 ' 901$ & 1987 & 46.8 & 53.2 & $81^{\prime} 058$ \\
\hline 1969 & 25.4 & 74.6 & $140^{\prime} 245$ & 1988 & 47.9 & 52.1 & $86 ' 548$ \\
\hline 1970 & 32.1 & 67.9 & $103^{\prime} 711$ & 1989 & 46.6 & 53.4 & $92 ' 989$ \\
\hline 1971 & 38.4 & 61.6 & $85^{\prime} 142$ & 1990 & 46.6 & 53.4 & $117 ' 711$ \\
\hline 1972 & 37.2 & 62.8 & $89^{\prime} 686$ & 1991 & 50.2 & 49.8 & $126 ' 645$ \\
\hline 1973 & 39.7 & 60.3 & $90 ' 118$ & 1992 & 56.6 & 43.4 & $128^{\prime} 183$ \\
\hline 1974 & 42.1 & 57.9 & $70^{\prime} 401$ & 1993 & 61.8 & 38.2 & $117^{\prime} 636$ \\
\hline 1975 & 52.6 & 47.4 & $54^{\prime} 223$ & 1994 & 61.1 & 38.9 & $103^{\prime} 613$ \\
\hline 1976 & 54.5 & 45.5 & $54^{\prime} 166$ & 1995 & 65.1 & 34.9 & $94^{\prime} 268$ \\
\hline 1977 & 58.5 & 41.5 & $61^{\prime} 141$ & 1996 & 62.7 & 37.3 & $79 ' 708$ \\
\hline 1978 & 54.9 & 45.1 & $57^{\prime} 340$ & 1997 & 65.1 & 34.9 & 72769 \\
\hline
\end{tabular}

Quelle: BFA (1998), eigene Berechnungen

Anmerkungen: Zuwanderung von Ausländern mit einer Jahresaufenthalts- oder Niederlassungsbewilligung (inkl. Umwandlungen von Saisonnier-in Nicht-Saisonnierbewilligungen)

Während sich die ausländischen Erwerbstätigen in den 50er und 60er Jahren, ähnlich der Zeit vor dem Ersten Weltkrieg, fast ausnahmslos aus den Anrainerstaaten der Schweiz rekrutierten (1950 belief sich dieser Anteil auf 95 Prozent, wobei die italienischen Arbeitskräfte mit einem Anteil von 60 Prozent klar dominierten), re-

49 Es ist allerdings zu beachten, dass in TAB. 3.2 lediglich Jahresaufenthalter und Niedergelassene berücksichtigt sind und damit die quantitativ bedeutsamen Kategorien der Saisonniers und Grenzgänger nicht erfasst sind (siehe TAB. 3.4). 
duzierte sich der Anteil der Ausländer aus den Nachbarländern bis 1970 auf weniger als 75 Prozent (siehe TAB. 3.3). 1997 stammten lediglich noch rund 52 Prozent der erwerbstätigen Ausländer aus den Anrainerstaaten der Schweiz. Ein ähnliches Bild ergibt sich bei der Betrachtung der ausländischen Wohnbevölkerung. Kamen bis 1960 noch 87 Prozent der Ausländer aus den Anrainerstaaten, waren es Ende 1997 nur noch rund 39 Prozent. Weitere 22 Prozent stammten aus anderen EUStaaten, insbesondere aus Portugal (10 Prozent) und Spanien (7 Prozent). Weitere 23 Prozent kamen aus dem ehemaligen Jugoslawien. Die restlichen 16 Prozent haben ihren Ursprung in anderen nicht-europäischen Staaten, insbesondere der Türkei (6 Prozent). Mit der zunehmenden Verlagerung der Herkunftsländer hat sowohl eine sprachliche wie auch kulturelle Pluralisierung der ausländischen Bevölkerung stattgefunden. HAUG (1995) diagnostizierte dementsprechend für die Schweiz einen Wandel vom Einwanderungsland zur multikulturellen Gesellschaft.

Tab. 3.3: Erwerbstätige ausländische Bevölkerung nach Staatsangehörigkeit, 1973-1997

\begin{tabular}{|c|c|c|c|c|c|c|c|c|c|c|}
\hline \multirow[t]{2}{*}{ Jahr } & \multirow{2}{*}{$\begin{array}{c}\text { Erwerbs- } \\
\text { tätige }\end{array}$} & \multicolumn{9}{|c|}{ Staatsangehörigkeit } \\
\hline & & $D$ & $I$ & $\boldsymbol{F}$ & $\boldsymbol{A}$ & $E$ & $\boldsymbol{P}$ & $E X J$ & $T$ & Rest \\
\hline 1973 & $406 ' 055$ & 7.0 & 46.5 & 4.8 & 2.7 & 18.6 & 0.9 & 6.4 & 3.4 & 9.8 \\
\hline 1974 & $439 ' 429$ & 7.3 & 47.5 & 4.6 & 2.9 & 17.3 & 0.9 & 6.3 & 3.6 & 9.6 \\
\hline 1975 & $459 ' 619$ & 8.1 & 49.4 & 4.8 & 3.2 & 15.3 & 0.8 & 5.5 & 3.3 & 9.5 \\
\hline 1976 & $466^{\prime} 420$ & 8.9 & 49.0 & 5.1 & 3.6 & 14.1 & 0.9 & 5.5 & 3.2 & 9.7 \\
\hline 1977 & $479 ' 164$ & 9.3 & 48.5 & 5.2 & 3.8 & 13.2 & 1.0 & 5.7 & 3.3 & 10.0 \\
\hline 1978 & $498^{\prime} 319$ & 9.3 & 48.7 & 5.0 & 4.0 & 12.6 & 1.1 & 5.8 & 3.4 & 10.1 \\
\hline 1979 & $498^{\prime} 841$ & 9.2 & 47.6 & 5.0 & 4.0 & 12.5 & 1.4 & 6.1 & 3.3 & 10.9 \\
\hline 1980 & $602^{\prime} 420$ & 10.5 & 43.5 & 11.5 & 4.3 & 10.5 & 1.4 & 5.4 & 3.4 & 9.5 \\
\hline 1981 & $636 ' 641$ & 10.2 & 42.9 & 11.7 & 1.6 & 10.2 & 1.8 & 6.0 & 3.6 & 12.1 \\
\hline 1982 & $647^{\prime} 946$ & 10.2 & 41.6 & 11.6 & 4.1 & 10.2 & 2.2 & 6.5 & 3.8 & 9.8 \\
\hline 1983 & $643^{\prime} 756$ & 10.1 & 41.2 & 11.5 & 4.1 & 10.4 & 2.4 & 6.7 & 3.8 & 9.8 \\
\hline 1984 & $655^{\prime} 684$ & 10.0 & 40.4 & 11.5 & 4.1 & 10.4 & 3.0 & 7.0 & 3.9 & 9.8 \\
\hline 1985 & $673 ' 404$ & 10.0 & 39.4 & 11.6 & 4.1 & 10.4 & 3.6 & 7.4 & 3.9 & 9.6 \\
\hline 1986 & 701 '528 & 10.1 & 38.2 & 11.9 & 4.0 & 10.2 & 4.3 & 7.8 & 3.9 & 9.5 \\
\hline 1987 & $735^{\prime} 728$ & 10.1 & 37.1 & 12.1 & 4.1 & 10.0 & 5.0 & 8.3 & 3.8 & 9.4 \\
\hline 1988 & $769^{\prime} 447$ & 10.4 & 35.7 & 12.7 & 4.1 & 9.7 & 5.4 & 8.7 & 3.8 & 9.4 \\
\hline 1989 & $813^{\prime} 158$ & 10.4 & 34.2 & 13.4 & 4.0 & 9.2 & 6.2 & 9.3 & 3.8 & 9.4 \\
\hline 1990 & $864^{\prime} 469$ & 10.7 & 32.2 & 13.7 & 4.2 & 8.8 & 7.0 & 10.2 & 3.9 & 9.3 \\
\hline 1991 & $888^{\prime} 775$ & 10.4 & 31.4 & 13.1 & 3.7 & 8.4 & 7.6 & 11.4 & 4.1 & 10.0 \\
\hline 1992 & $893^{\prime} 479$ & 10.1 & 30.5 & 12.6 & 3.5 & 7.9 & 8.2 & 12.8 & 4.1 & 10.2 \\
\hline 1993 & $898^{\prime} 967$ & 9.8 & 29.8 & 12.1 & 3.3 & 7.7 & 9.0 & 14.1 & 4.2 & 10.1 \\
\hline
\end{tabular}


Tab. 3.3: Erwerbstätige ausländische Bevölkerung nach Staatsangehörigkeit, 1973-1997 (Fortsetzung)

\begin{tabular}{|l|l|rrrrrrrrr|}
\hline 1994 & $911^{\prime} 633$ & 9.7 & 29.0 & 11.8 & 3.2 & 7.4 & 9.5 & 15.1 & 4.1 & 10.2 \\
1995 & $895^{\prime} 735$ & 9.9 & 28.2 & 12.0 & 3.2 & 7.2 & 9.7 & 15.4 & 4.0 & 10.5 \\
1996 & $869^{\prime} 701$ & 10.2 & 27.4 & 12.1 & 3.1 & 7.0 & 9.8 & 15.8 & 4.0 & 10.6 \\
1997 & $847^{\prime} 447$ & 10.5 & 26.7 & 12.1 & 3.1 & 6.7 & 9.8 & 16.3 & 3.9 & 10.8 \\
\hline
\end{tabular}

Quelle: BFA (1998), eigene Berechnungen

Anmerkungen: Die erwerbstätige ausländische Bevölkerung umfasst Jahresaufenthalter, Niedergelassene, Saisonarbeiter und Grenzgänger; die Angaben beziehen sich auf Ende Dezember

$D=$ Deutschland, $I=$ Italien, $F=$ Frankreich, $A=$ Österreich, $E=$ Spanien, $P=$ Portugal, EXJ = Ex-Jugoslawien, $T=$ Türkei

Analog zur Verlagerung von den traditionellen zu neuen Zuwanderungsgebieten, lässt sich auch hinsichtlich der Aufenthaltskategorien eine Veränderung in der $\mathrm{Zu}$ sammensetzung der ausländischen Erwerbstätigen feststellen (siehe TAB. 3.4). Seit den 70er Jahren hat der Anteil der Niedergelassenen an der ausländischen Bevölkerung massiv zugenommen, während der Anteil der Jahresaufenthalter stark rückläufig ist. Zwischen 1970 und 1997 hat nahezu eine Verdreifachung der Zahl der erwerbstätigen Niedergelassenen von 182'000 auf 520'000 Personen stattgefunden. Im gleichen Zeitraum verringerte sich die Zahl der erwerbstätigen Jahresaufenthalter von $410^{\prime} 000$ auf $172^{\prime} 000$ Personen, d.h. um rund 60 Prozent. Eine ähnliche Entwicklung lässt sich bei den Grenzgängern und Saisonniers erkennen. Wie aus TAB. 3.4 hervorgeht, hat die Zahl der Grenzgänger zu Lasten der Saisonniers stark zugenommen.

Diese Entwicklungen sind im wesentlichen auf zwei Faktoren zurückzuführen. Erstens haben viele Ausländer mit Jahresaufenthaltsbewilligungen aufgrund ihrer langen Aufenthaltsdauer, von ihrem Recht auf eine Niederlassungsbewilligung Gebrauch gemacht. Gleichzeitig hatte die Globalplafonierung ab 1970 sowie die rückläufige wirtschaftliche Entwicklung Mitte der 70er Jahre und zu Beginn der 80er Jahre einen massiven Rückgang der Zahl der Jahresaufenthalter zur Folge. Zweitens hat die Tatsache, dass Grenzgänger- im Gegensatz zu Saisonnierbewilligungen nicht der Kontingentierung unterstehen, dazu geführt, dass in den Grenzregionen für viele Unternehmen Anreize entstanden sind, Grenzgänger anstelle von Saisonniers zu beschäftigen, was die gegenläufige Entwicklung bei Grenzgängern und Saisonniers erklärt.

Zusammenfassend kann somit festgehalten werden, dass die Zusammensetzung der ausländischen Bevölkerung immer stärker dauerhaften Charakter besitzt. Im Jahre 1997 waren rund 94 Prozent der in der Schweiz lebenden Bevölkerung der 'ständigen ausländischen Wohnbevölkerung' zuzurechnen, annähernd 69 Prozent als Niedergelassene und 25 Prozent als Jahresaufenthalter. In Übereinstimmung 
dazu lässt sich zeigen, dass lediglich rund 25 Prozent der Ausländer seit weniger als 5 Jahren in der Schweiz leben. 50

Tab. 3.4: Ausländische Erwerbstätige nach Aufenthaltskategorien, 1970 - 1997

\begin{tabular}{|c|c|c|c|c|c|c|c|c|c|}
\hline Jahr & $\begin{array}{c}B-B e- \\
\text { willigung }\end{array}$ & $\begin{array}{c}C-B e- \\
\text { willigung }\end{array}$ & $\begin{array}{c}A-B e- \\
\text { willigung }\end{array}$ & $\begin{array}{c}G-B e- \\
\text { willigung }\end{array}$ & Jahr & $\begin{array}{c}B-B e- \\
\text { willigung }\end{array}$ & $\begin{array}{c}C-B e- \\
\text { willigung }\end{array}$ & $\begin{array}{c}A-B e- \\
\text { willigung }\end{array}$ & $\underset{\text { willigung }}{G-B e-}$ \\
\hline 1970 & 410 & 183 & 155 & 74 & 1984 & 120 & 419 & 101 & 106 \\
\hline 1971 & 370 & 217 & 181 & 87 & 1985 & 123 & 426 & 103 & 113 \\
\hline 1972 & 342 & 254 & 197 & 92 & 1986 & 132 & 435 & 110 & 122 \\
\hline 1973 & 308 & 287 & 194 & 98 & 1987 & 144 & 444 & 115 & 133 \\
\hline 1974 & 275 & 319 & 152 & 103 & 1988 & 155 & 453 & 121 & 150 \\
\hline 1975 & 225 & 327 & 86 & 85 & 1989 & 169 & 463 & 120 & 167 \\
\hline 1976 & 189 & 327 & 61 & 77 & 1990 & 170 & 500 & 122 & 181 \\
\hline 1977 & 164 & 328 & 67 & 80 & 1991 & 169 & 533 & 116 & 176 \\
\hline 1978 & 145 & 344 & 84 & 84 & 1992 & 174 & 543 & 93 & 165 \\
\hline 1979 & 129 & 362 & 96 & 89 & 1993 & 177 & 549 & 72 & 156 \\
\hline 1980 & 127 & 374 & 110 & 95 & 1994 & 187 & 554 & 61 & 152 \\
\hline 1981 & 129 & 386 & 120 & 110 & 1995 & 186 & 543 & 54 & 151 \\
\hline 1982 & 129 & 397 & 116 & 108 & 1996 & 180 & 529 & 45 & 147 \\
\hline 1983 & 125 & 405 & 100 & 105 & 1997 & 172 & 521 & 31 & 142 \\
\hline
\end{tabular}

Quelle: BFA (1998), eigene Berechnungen

Anmerkungen: Angaben (in I'O00) beziehen sich auf Ende Dezember, für Saisonniers auf Ende August B-Bewilligung: Jahresaufenthaltsbewilligung, C-Bewilligung: Niederlassungsbewilligung, ABewilligung: Saisonnierbewilligung, G-Bewilligung: Grenzgängerbewilligung

Die schweizerische Ausländerpolitik, die in ihrer Grundkonzeption vor allem als Arbeitsmarktpolitik zu verstehen ist, hat aus diesen Gründen einen Grossteil ihres Handlungsspielraums und ihrer Steuerungsfunktion eingebüsst. Dieser Umstand sowie die Tatsache, dass im Zeitverlauf eine Verlagerung sowohl hinsichtlich der Migrationsmotive als auch der Herkunftsländer der Ausländer stattgefunden hat, müssen bei der Analyse der Arbeitsmarktperformance der Ausländer berücksichtigt werden, da davon auszugehen ist, dass beide Faktoren einen Einfluss auf die Performance der Einwanderer besitzen.

\subsection{BESCHÄFTIGUNGSPERFORMANCE VON EINWANDERERN}

Die Beschäftigungsperformance bildet zusammen mit der Einkommensperformance, die im nächsten Abschnitt betrachtet wird, die zentrale Messgrösse zur Beurteilung der Arbeitsmarktperformance der ausländischen Arbeitnehmer auf

50 FISCHER /STRAUBHAAR (1996b). 
dem schweizerischen Arbeitsmarkt. Es werden daher im folgenden einige wichtige Indikatoren zur Beurteilung der Beschäftigungs- und Einkommensperformance betrachtet, die erste Hinweise auf die Performance der Ausländer auf dem schweizerischen Arbeitsmarkt liefern.

Tab. 3.5: Erwerbsquoten von Ausländern und Schweizern, 1960 - 1997

\begin{tabular}{|c|cc|c|ccc|c|cc|}
\hline Jahr & Ausländer & Schweizer & Jahr & Ausländer & Schweizer & \multicolumn{2}{|c|}{ Jahr } & Ausländer & Schweizer \\
\hline 1960 & 73.2 & 47.1 & 1973 & 57.9 & 46.5 & 1986 & 59.1 & 48.9 \\
1961 & 75.7 & 47.2 & 1974 & 55.9 & 46.8 & 1987 & 60.6 & 49.4 \\
1962 & 76.6 & 47.1 & 1975 & 55.0 & 45.3 & 1988 & 61.9 & 50.0 \\
1963 & 75.1 & 46.5 & 1976 & 54.1 & 45.2 & 1989 & 62.9 & 50.5 \\
1964 & 73.5 & 46.4 & 1977 & 53.5 & 45.6 & 1990 & 64.1 & 51.1 \\
1965 & 70.3 & 46.0 & 1978 & 53.7 & 45.8 & 1991 & 64.9 & 51.8 \\
1966 & 67.6 & 45.6 & 1979 & 55.2 & 45.9 & 1992 & 64.1 & 51.3 \\
1967 & 65.7 & 45.4 & 1980 & 55.3 & 46.5 & 1993 & 62.3 & 51.6 \\
1968 & 64.9 & 45.2 & 1981 & 56.4 & 47.1 & 1994 & 61.1 & 51.2 \\
1969 & 63.4 & 45.3 & 1982 & 56.7 & 47.1 & 1995 & 61.2 & 50.9 \\
1970 & 63.2 & 45.5 & 1983 & 55.9 & 47.5 & 1996 & 60.6 & 51.4 \\
1971 & 60.4 & 45.9 & 1984 & 57.4 & 47.7 & 1997 & 60.1 & 51.6 \\
1972 & 58.9 & 46.2 & 1985 & 57.5 & 48.4 & & & \\
\hline
\end{tabular}

Quelle: BFS (1998a), eigene Berechnungen

Anmerkungen: Enwerbsquote $=$ Enwerbstätige und Arbeitslose (ohne Saisonniers, Grenzgänger, Kurzaufent halter, Asylbewerber und internationale Funktionäre) in Prozenten der ständigen Wohnbevölkerung. Die Berechnung der Erwerbsquoten basiert auf den Daten der Erwerbstätigenstatistik Erfasst werden nach dem Inlandskonzept alle erwerbstätigen Personen mit einer Mindestarbeitszeit von 6 Std./Woche

In TAB. 3.5 sind die Erwerbsquoten der In- und Ausländer für den Zeitraum von 1960 bis 1997 abgebildet. Es zeigt sich, dass die Erwerbsquoten der Ausländer substantiell über jenen der Schweizer liegen. Gleichzeitig ist erkennbar, dass im Zeitverlauf eine partielle Konvergenz der Erwerbsquoten der In- und Ausländer stattgefunden hat. Diese Entwicklung war begleitet von einer Verlagerung der Zuwanderung von der Arbeits- zur Familien- und Flüchtlingsmigration. Der Rückgang der Erwerbsquote der Ausländer lässt sich somit einerseits auf die niedrigeren Erwerbsquoten der Frauen zurückführen sowie andererseits auf die Tatsache, dass sich Ausländer der Zweiten Generation oftmals noch in der Ausbildung befinden. Ferner ist zu vermuten, dass die Erwerbsquote von Flüchtlingen aufgrund der z.T. beträchtlichen Integrationshürden zumindest anfänglich vergleichsweise gering ausfällt. Die steigende Erwerbsquote der Schweizer hängt 
schliesslich mit der zunehmenden Erwerbstätigkeit der Frauen seit den 80er Jahren zusammen. ${ }^{51}$

Tab. 3.6: Arbeitslosenquoten von Schweizern und Ausländern, 1976- 1997

\begin{tabular}{|c|ccc|c|ccc|}
\hline Jahr & Total & Ausländer & Schweizer & Jahr & Total & Ausländer & Schweizer \\
\hline 1976 & 0.7 & 0.8 & 0.7 & 1987 & 0.8 & 1.5 & 0.5 \\
1977 & 0.4 & 0.4 & 0.4 & 1988 & 0.6 & 1.4 & 0.5 \\
1978 & 0.4 & 0.3 & 0.4 & 1989 & 0.6 & 1.1 & 0.4 \\
1979 & 0.3 & 0.4 & 0.3 & 1990 & 0.5 & 0.9 & 0.4 \\
1980 & 0.2 & 0.3 & 0.2 & 1991 & 1.1 & 2.1 & 0.8 \\
1981 & 0.2 & 0.3 & 0.2 & 1992 & 2.5 & 4.5 & 2.0 \\
1982 & 0.4 & 0.7 & 0.4 & 1993 & 4.5 & 7.8 & 3.5 \\
1983 & 0.9 & 1.5 & 0.7 & 1994 & 4.7 & 8.4 & 3.7 \\
1984 & 1.0 & 1.8 & 0.9 & 1995 & 4.2 & 8.0 & 3.2 \\
1985 & 1.0 & 1.6 & 0.7 & 1996 & 4.7 & 9.3 & 3.3 \\
1986 & 0.6 & 0.8 & 0.5 & 1997 & 5.2 & 10.7 & 3.6 \\
\hline
\end{tabular}

Quelle: BFS (1998b), eigene Berechnungen

Anmerkungen: Die Berechnung der Arbeitslosenquoten beruht auf Jahresdurchschnittswerten Arbeitslosenquote $=$ Zahl durchschnittlich eingeschriebener Arbeitsloser $/$ Zahl der Erwerbspersonen gemäss Volkszählung der Jahre 1970, 1980 und 1990.

In TAB. 3.6 sind die Arbeitslosenquoten der In- und Ausländer für den Zeitraum zwischen 1976 und 1997 dargestellt. Es zeigt sich, dass die Arbeitslosenquote der Ausländer praktisch während des gesamten Betrachtungszeitraums erheblich über jener der Schweizer lag.52 Aus TAB. 3.6 geht ebenfalls hervor, dass sich die Phase erhöhter Arbeitslosigkeit in jeder Rezession der letzten 20 Jahre verstärkte und verlängerte. Es ist somit anzunehmen, dass seit Beginn der 80er Jahre hinsichtlich der Arbeitslosigkeit ein sog. 'Hystereseeffekt' besteht. Dies bedeutet, dass die Arbeitslosenquoten seit den $80 \mathrm{er}$ Jahren jeweils auf einem rezessionsbedingt höheren Niveau verharrt sind und während der nachfolgenden Aufschwungsphasen nicht mehr vollständig zurückgegangen sind. Im Zeitverlauf hat daher eine kontinuierliche Erhöhung der Arbeitslosenquoten stattgefunden, die seit 1991 besonders ausgeprägt ist.

Dieser Hystereseeffekt lässt sich einerseits auf den zunehmenden Leistungsausbau im Rahmen der Arbeitslosenversicherung während der vergangenen 20 Jahre zurückführen sowie andererseits auf rezessionsbedingte Strukturbereinigungen, die

51 Siehe MÜLLER (1991).

52 Trotz der im Vergleich zu den anderen OECD-Staaten tiefen Arbeitslosenquote bleibt festzuhalten, dass die Zahl der Arbeitslosen zwischen 1990 und 1997 um 1038 Prozent zugenommen hat. 
zur Freisetzung von Arbeitskräften geführt haben. Von Arbeitslosigkeit sind in besonderem Masse diejenigen ausländischen Arbeitskräfte betroffen, die u.a. aufgrund ihrer im Mittel tieferen Qualifikationen in den strukturschwachen Branchen des sekundären Sektors tätig sind.

Diese Überlegungen bestätigen sich auch mit Blick auf TAB. 3.7, in der die erwerbstätige Bevölkerung nach sozio-professionellen Kategorien gegliedert ist. Es zeigt sich, dass die ausländischen Erwerbstätigen vor allem in den Kategorien der ungelernten Arbeiter und Angestellten sowie in den qualifizierten manuellen $\mathrm{Be}-$ rufen konzentriert und nur in geringem Masse selbständig oder in freien Berufen tätig sind..$^{53}$ Gleichzeitig geht aus TAB. 3.7 hervor, dass ebenfalls ein signifikanter Teil der Ausländer im oberen Segment der sozio-professionellen Kategorien zu finden ist. Diese Heterogenität verdeutlicht die Notwendigkeit einer differenzierten Vorgehensweise zur Analyse der Arbeitsmarktperformance.

Tab. 3.7: Erwerbstätige Bevölkerung nach sozio-professionellen Kategorien, 1990

\begin{tabular}{|l|c|cccccccc|}
\hline Nationalität & Jahr & $\mathbf{1}$ & $\mathbf{2}$ & $\mathbf{3}$ & $\mathbf{4}$ & $\mathbf{5}$ & $\mathbf{6}$ & $\mathbf{7}$ & $\boldsymbol{8}$ \\
\hline Ausländer & 1980 & 12.6 & 9.4 & 6.7 & 14.3 & 14.5 & 9.4 & 18.2 & 29.5 \\
& 1990 & 12.6 & 8.5 & 9.2 & 14.9 & 14.1 & 13.1 & 23.7 & 45.9 \\
\hline \multirow{2}{*}{ Schweizer } & 1980 & 87.4 & 90.6 & 93.3 & 85.7 & 85.5 & 90.6 & 81.8 & 70.5 \\
& 1990 & 87.4 & 91.5 & 90.8 & 85.1 & 85.9 & 86.9 & 76.3 & 54.1 \\
\hline
\end{tabular}

Quelle: BFS, Eidg. Volkszählung, eigene Berechnungen

Anmerkungen: Sozio-professionelle Kategorien: I=Oberstes Management, 2=Freie Berufe (Selbständige Berufe mit Hochschulabschluss), 3=Andere Selbständige (Selbständige Berufe ohne Hochschulabschluss), 4=Akademische Berufe und oberes Kader, 5=Intermediäre Berufe,

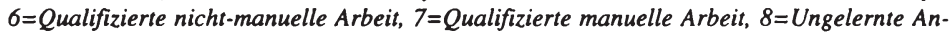
gestellte und Arbeiter

In TAB. 3.8 finden sich schliesslich die Beschäftigungsanteile der Ausländer innerhalb der verschiedenen Wirtschaftszweige. Es zeigt sich, dass die ausländischen Erwerbstätigen während der letzten drei Jahrzehnte vor allem in der Verarbeitenden Produktion, im Baugewerbe sowie im Handel und Gastgewerbe konzentriert waren. Die Zahlen in TAB. 3.8 verdeutlichen aber auch, dass im Zeitverlauf eine Verlagerung hin zu den Wirtschaftszweigen des Dienstleistungssektors stattgefunden hat. Gleichzeitig geht aus TAB. 3.8 hervor, dass die Ausländer insbesondere in der Land- und Forstwirtschaft, in der Energie- und Wasserversorgung, der Verkehrs- und Nachrichtenübermittlung sowie im öffentlichen Sektor nur schwach vertreten sind. Es liegt somit die Vermutung nahe, dass die Beschäf-

53 Dieses Ergebnis ist allerdings im wesentlich darauf zurückzuführen, dass die Landwirtschaft mit ihrem hohen Anteil an selbständig Erwerbstätigen eine fast ausschliesslich von Schweizern besetzte Domäne ist. Zudem wurde in Kapitel 2 bereits darauf hingewiesen, dass die selbständige Erwerbstätigkeit für Ausländer bewilligungspflichtig ist. 
tigungsanteile der ausländischen Erwerbstätigen vor allem in denjenigen Wirtschaftszweigen hoch sind, die ein tiefes Qualifikationsprofil erfordern.

Tab. 3.8: Erwerbstätige Bevölkerung nach Wirtschaftszweigen, 1970-1990

\begin{tabular}{|l|c|ccccccccc|}
\hline Nationalität & Jahr & $\mathbf{I}$ & $\mathbf{2}$ & $\mathbf{3}$ & $\mathbf{4}$ & $\mathbf{5}$ & $\mathbf{6}$ & $\mathbf{7}$ & $\mathbf{8}$ & $\boldsymbol{9}$ \\
\hline Ausländer & 1970 & 4.4 & 4.0 & 28.9 & 36.8 & 18.6 & 6.8 & 12.8 & 20.8 & 8.5 \\
& 1980 & 3.5 & 4.8 & 24.4 & 34.0 & 17.2 & 7.5 & 12.0 & 16.3 & 9.2 \\
& 1990 & 7.1 & 6.5 & 28.9 & 39.4 & 23.3 & 12.0 & 15.8 & 17.7 & 9.5 \\
\hline \multirow{5}{*}{ Schweizer } & 1970 & 95.6 & 96.0 & 71.1 & 63.2 & 81.4 & 93.2 & 87.2 & 79.2 & 91.5 \\
& 1980 & 96.5 & 95.2 & 75.6 & 66.0 & 82.8 & 92.5 & 88.0 & 83.7 & 90.8 \\
& 1990 & 92.9 & 93.5 & 71.1 & 60.6 & 76.7 & 88.0 & 84.2 & 82.3 & 90.5 \\
\hline
\end{tabular}

Quelle: BFS, Eidg. Volkszählung, eigene Berechnungen

Anmerkungen: Wirtschaftszweige: $1=$ Land- und Forstwirtschaft, 2=Energie- und Wasserversorgung, Bergbau, 3=Verarbeitende Produktion, 4=Baugewerbe, 5=Handel, Gastgewerbe, Reperaturgewerbe, $6=V e r k e h r$, Nachrichtenübermittlung, 7=Banken, Versicherungen, Immobilien, $8=$ Sonstige Dienstleistungen (Gesundheits-, Unterrichts und Forschungswesen, etc.), $9=$ Öffentliche Verwaltung

\subsection{EINKOMMENSPERFORMANCE VON EINWANDERERN}

Neben der Beschäftigungsperformance stellt die Einkommensperformance die zweite zentrale Messgrösse zur Beurteilung der Arbeitsmarktperformance dar. Da für die Schweiz erst seit Einführung der SAKE im Jahre 1991 Einkommensdaten für In- und Ausländer zur Verfügung stehen, ist eine intertemporale Betrachtung der Einkommensentwicklung nicht möglich. In Übereinstimmung mit den Ergebnissen der Beschäftigungsperformance sind deutliche Unterschiede in den Einkommensniveaus zwischen In- und Ausländern erkennbar. Aus TAB. 3.9 geht hervor, dass die Ausländer deutlich höhere Anteile in den unteren Einkommenssegmenten aufweisen als die Inländer. Bemerkenswert ist ebenfalls der hohe Prozentsatz der Befragten, die keine Angaben über ihre Erwerbseinkommen gemacht haben. Dies hängt damit zusammen, dass die Befragungen im Rahmen der SAKE auf freiwilliger Basis stattfinden. ${ }^{54}$

Tab. 3.9: Erwerbstätige nach jährlichem Bruttoerwerbseinkommen in CHF, 1995

\begin{tabular}{|l|cccccccc|}
\hline Nationalität & $\begin{array}{c}\text { Bis } \\
26^{\prime} 000\end{array}$ & $\begin{array}{c}26^{\prime} 001- \\
39^{\prime} 000\end{array}$ & $\begin{array}{c}39^{\prime} 001- \\
52^{\prime} 000\end{array}$ & $\begin{array}{c}52^{\prime} 001- \\
65^{\prime} 000\end{array}$ & $\begin{array}{c}65^{\prime} 001- \\
7^{\prime} 000\end{array}$ & $\begin{array}{c}78^{\prime} 001- \\
\mathbf{1 0 4}^{\prime} 000\end{array}$ & $\begin{array}{c}\text { Über } \\
104^{\prime} 000\end{array}$ & $\begin{array}{c}\text { Keine } \\
\text { Angabe }\end{array}$ \\
\hline Ausländer & 1.8 & 8.2 & 17.3 & 23.3 & 14.1 & 8.4 & 7.2 & 19.6 \\
Schweizer & 2.7 & 3.5 & 11.4 & 16.2 & 16.1 & 17.1 & 13.3 & 19.7 \\
\hline
\end{tabular}

Quelle: Schweizerische Arbeitskräfteerhebung (SAKE), eigene Berechnungen

54 Nähere Angaben zur Struktur und Erhebungsmethode der SAKE finden sich in Kapitel 8. 


\subsection{FAZIT}

Die Ausführungen dieses Kapitels beschliessen den ersten, einführenden Teil mit einer statistischen Dokumentation der Ausländerbeschäftigung. Nach der Diskussion der historischen Erfahrungen der Zuwanderung in die Schweiz in Kapitel 3, besteht der wesentliche Beitrag dieses Kapitels darin, dass er erste Hinweise auf die Arbeitsmarktperformance der Ausländer im Vergleich zu den Schweizern liefert. Die Analyse zeigt, dass zwischen In- und Ausländern sowohl hinsichtlich der Beschäftigungs- als auch der Einkommensperformance Unterschiede bestehen. Zum einen liegt die Arbeitslosenquote für Ausländer markant über jener der Schweizer, und zum anderen sind die Ausländer weit stärker in den unteren Einkommenssegmenten vertreten als die Inländer. Die Untersuchungen dieses Kapitels haben gezeigt, dass diese Diskrepanz zumindest teilweise durch Unterschiede in den sozio-demographischen Charakteristika zwischen In- und Ausländern bedingt ist.

Die Ergebnisse dieses Kapitels enthalten klare Hinweise darauf, dass eine differenzierte Analyse notwendig ist, um aussagekräftige Ergebnisse zur Arbeitsmarktperformance gewährleisten zu können. Aus diesem Grund wird für die empirische Analyse des dritten Teils eine differenzierte Vorgehensweise gewählt, indem geschlechts-, nationalitäten- und kohortenspezifischen Unterschieden Rechnung getragen wird. Durch diese Vorgehensweise lässt sich der Heterogenität des Untersuchungsgegenstandes Rechnung tragen. 


\section{TEIL 2 (THEORIE): \\ THEORETISCHE ANALYSE DER \\ ARBEITSMARKTPERFORMANCE}

\section{4. ÖKONOMISCHE ANSÄTZE ZUR ERKLÄRUNG VON MIGRATION}

Für das Entstehen von Migration sind eine Vielzahl verschiedener Faktoren verantwortlich. $\mathrm{Zu}$ diesen zählen ökonomische Faktoren wie die Aussicht auf eine bessere Beschäftigungssituation, bessere Arbeitsbedingungen oder höhere Löhne im Zielland, demographische Faktoren wie der Umfang und die Altersverteilung der Arbeitsbevölkerung in den Herkunftsländern sowie politische Faktoren wie politische Verfolgung oder Repression im Herkunftsland. Das Zusammenspiel dieser Faktoren führt zu einem Migrationspotential und daraus resultierender angebotsbestimmter Migration (Push-Migration). Die Existenz migrationswilliger Wirtschaftssubjekte stellt jedoch lediglich eine notwendige, nicht aber eine hinreichende Bedingung für das Entstehen von Migration dar, da die effektiv stattfindende Zuwanderung sowohl von der Migrationsnachfrage nach ausländischen Arbeitskräften als auch von der Migrationspolitik des Aufnahmelandes abhängt. ${ }^{55}$ In ABB. 4.1 findet sich eine Zusammenstellung der wichtigsten Ansätze zur Erklärung von Migration, die in diesem Kapitel diskutiert werden. ${ }^{56}$

Migration wird in diesem Kapitel als Reaktion der Haushalte auf räumliche Nutzenunterschiede verstanden und daher im Rahmen von Ungleichgewichtsansätzen modelliert. Neben diesen wurden in den letzten 20 Jahren alternative Modelle entwickelt, die auf der Annahme basieren, dass Migration primär als Reaktion auf Veränderungen des Angebots- und Nachfrageverhaltens der Wirtschaftssubjekte zu verstehen ist. Diese sog. Gleichgewichtsansätze gehen davon aus, dass die lokalen Arbeitsmärkte sowie der Migrationsprozess selbst effizient sind und daher gilt, dass "... any significant spatial differences in utility that arise are quickly arbitraged through migration thereby promptly restoring equilibrium (i.e. compensating) differentials in the spatial pattern of economic opportunities." 57 Die Persistenz räumlicher Nutzenunterschiede im Ungleichgewichtsansatz im Vergleich

\footnotetext{
55 Auf diese Zusammenhänge wird in Kapitel 5.4 ausführlich eingegangen.

56 In den vergangenen Jahren sind verschiedene Übersichtsartikel zu diesem Thema erschienen. Siehe BAUER/ZimMERMANN (1995b), GREENWOOD (1985), MASSEY ET AL. (1993), MOLHO (1986), SHIELDS/SHIELDS (1989) und STRAUBHAAR (1988).
}

57 HUNT (1993: 342). 
zum Gleichgewichtsansatz wird im wesentlichen durch langsame Anpassungsprozesse auf dem Arbeitsmarkt erklärt. 58

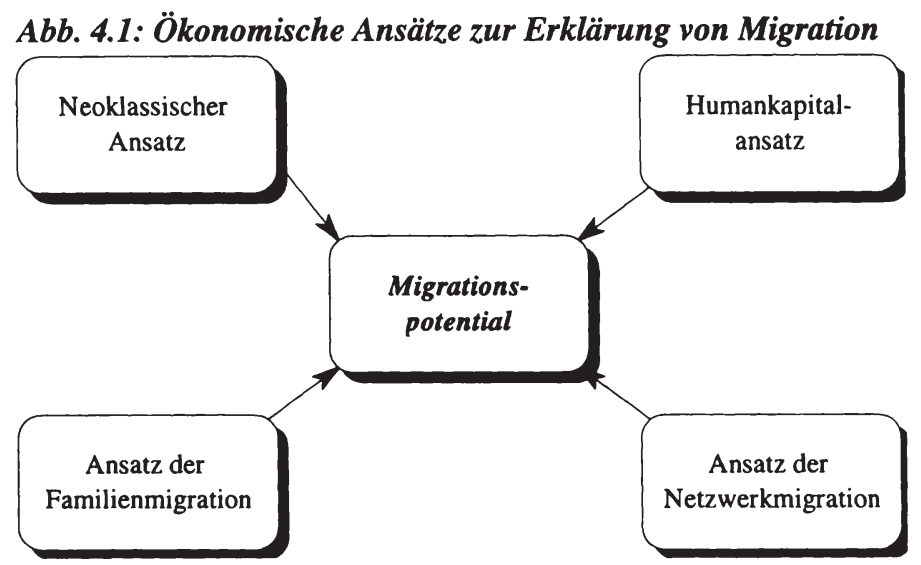

Quelle: Eigene Darstellung

Ziel dieses Kapitels ist es, die wichtigsten Faktoren zu erörtern, die für das Entstehen von angebotsbestimmter Migration verantwortlich sind. Diese Analyse soll erste Hinweise auf die Zusammensetzung sowie die sozio-ökonomischen bzw. sozio-demographischen Charakteristika der Einwanderer erbringen, die Einfluss auf die Performance der einzelnen Wirtschaftssubjekte besitzen.

\subsection{DER NEOKLASSISCHE ANSATZ}

Der neoklassische Ansatz kann bis auf SMTTH (1776) und RAVENSTEIN (1889) zurückverfolgt werden..$^{59} \mathrm{Er}$ basiert auf der Annahme, dass die Wirtschaftssubjekte die Zielsetzung verfolgen, ihre Nutzenfunktion unter Berücksichtigung der jeweiligen Budgetrestriktion zu maximieren, so dass der Lohnsatz bzw. das Einkommen die zentralen Parameter darstellen. Im Rahmen des neoklassischen Ansatzes wird Migration als Reaktion auf geographische Lohn- und Einkommensunterschiede verstanden, die auf divergierende Faktorausstattungen zwischen Regionen oder Ländern zurückzuführen sind. Lohn- bzw. Einkommensdifferentiale zwischen Ländern oder Regionen sind daher die Hauptdeterminanten für das Entstehen von Migrationsbewegungen. Wird im Rahmen eines Zwei-Länder-/ZweiFaktoren-Modells unterstellt, dass die erste Region eine grössere Arbeitsausstat-

58 Empirische Evidenz zur Gültigkeit dieser beiden Ansätze findet sich in CoLLINS (1991) und GABRIEU/SHACK-MARQUEZ/WASCHER (1993).

59 Für eine kritische Diskussion des neoklassischen Ansatzes siehe STRAUBHAAR (1988). 
tung aufweist und die zweite eine grössere Kapitalausstattung, so wird aufgrund der höheren Löhne in der zweiten Region eine Migrationsbewegung von der Tieflohn- in die Hochlohnregion stattfinden.

Migrationsbewegungen bestehen dementsprechend so lange, bis die Lohndifferentiale zwischen den beiden Regionen lediglich den Transaktionskosten entsprechen. HICKS bemerkte in diesem Zusammenhang, dass "... differences in net economic advantages chiefly in wages are the main causes of migration". ${ }^{60}$ Daraus folgt, dass die Migrationsbewegungen umso stärker ausfallen werden, je grösser die Lohndifferentiale bei gegebenen Transaktionskosten sind. Diese Kosten lassen sich näherungsweise mit der Distanz zwischen dem Aus- und dem Einwanderungsland approximieren, wobei zwischen der Distanz und der Migrationswahrscheinlichkeit ein inverser Zusammenhang besteht. Die Transaktionskosten stimmen dabei im neoklassischen Modell weitgehend mit den Transportkosten überein.

Die zentrale Schwachstelle des neoklassischen Ansatzes besteht darin, dass er keine Erklärung dafür zu geben vermag, warum trotz sinkender Transportkosten und gleichzeitig teilweise erheblicher internationaler Lohnunterschiede lediglich geringfügige internationale Migrationsbewegungen stattfinden. ${ }^{61}$ Die Fokussierung auf Realeinkommensunterschiede führt zu einer Vernachlässigung anderer zentraler Faktoren, welche die Migrationsentscheidung der Wirtschaftssubjekte beeinflussen. ${ }^{62}$ Diese Faktoren, die eine zusätzliche Verringerung der Migrationswahrscheinlichkeit implizieren, lassen sich im neoklassischen Modell als Zusatzkosten zu den Transaktionskosten interpretieren.

Im speziellen werden im neoklassischen Ansatz erstens berufsspezifische Faktoren, etwa gute Arbeitsbedingungen oder das Arbeitsumfeld, die sich nicht direkt in den Löhnen widerspiegeln, nicht in die Migrationsentscheidung miteinbezogen. Allgemeiner kann in diesem Zusammenhang davon ausgegangen werden, dass Wirtschaftssubjekte ortsspezifische 'Insider'-Vorteile beruflicher und nicht-beruflicher Art akkumulieren, die sich international nicht transferieren lassen. ${ }^{63}$ Zweitens werden mit der Fokussierung auf gegenwärtig bestehende Realeinkommensunterschiede intertemporale Überlegungen ausgeklammert, d.h. Erwartungen über die zukünftige Einkommensentwicklung werden nicht berücksichtigt. Drittens ist $\mathrm{zu}$ erwarten, dass Migranten keine vollständigen Informationen über die $\mathrm{Zu}$ kunftsperspektiven im Zielland besitzen und sich daher ihre Migrationswahrscheinlichkeit im Falle von Risikoaversität verringert. Viertens ist zu vermuten, dass migrationswillige Wirtschaftssubjekte die Wahrscheinlichkeit, im Zielland

\footnotetext{
60 HICKS (1932: 76).

61 STRAUBHAAR (1986: 838), STARK (1984a).

62 STRAUBHAAR (1988).

63 Siehe MALMBERG/FISCHER (1997).
} 
arbeitslos zu werden, in ihrer Migrationsentscheidung mitberücksichtigen werden. Im neoklassischen Modell wird Arbeitslosigkeit ausgeklammert, da flexible Löhne unterstellt werden und sich der Arbeitsmarkt daher per definitionem immer im Gleichgewicht befindet. ${ }^{64}$

\subsection{DER HumanKapitalansatz}

Der Humankapitalansatz, der auf BECKER (1962) zurückgeht, wurde von SJAASTAD (1962) in die Migrationsforschung eingeführt. Im Rahmen dieses Ansatzes, der in der Migrationsforschung zu den wichtigsten und einflussreichsten zählt, wird Migration als Investitionsentscheidung verstanden. Migration kann in diesem Kontext mit einer Kapitalanlage verglichen werden, da zu Beginn der Investition, d.h. mit der Wanderung, die Wanderungskosten sowie ein eventuell niedrigerer Anfangslohn, das Einkommen reduziert, und die Erträge, d.h. ein höheres Einkommen, bessere Lebensbedingungen und andere Vorteile erst allmählich anfallen.

Die Wirtschaftssubjekte berechnen in Abhängigkeit ihrer Fähigkeiten den abdiskontierten Gegenwartswert der erwarteten Erträge in jeder Region. Migration findet dann statt, wenn die abdiskontierten Erträge in der Zielregion abzüglich der Kosten der Wanderung grösser sind als die abdiskontierten Erträge im Herkunftsland. Mathematisch lässt sich dieser Zusammenhang wie folgt darstellen:65

$$
v(0)=\int_{t=0}^{n}\left(P_{t} \cdot Y_{t}^{D}-Y_{t}^{0}\right) \cdot e^{-t t} d t-C(0)
$$

64 Das Grundmodell des neoklassischen Ansatzes ist in verschiedener Hinsicht erweitert worden. So gaben TODARO $(1968,1969)$ und HARRIS/TODARO (1970) zur Erklärung der LandStadt-Migration die neoklassische Annahme der Vollbeschäftigung auf und erweiterten die Nutzenfunktion der Migranten um die Erwerbstätigkeitswahrscheinlichkeit in der Zielregion [siehe TODARO (1980)]. Diese Erweiterung erlaubt es, die z.T. substantiellen Migrationsbewegungen in unterentwickelten Regionen von ländlichen in städtische Gebiete, trotz mangelnder Beschäftigungsperspektiven, zu erklären. Im Gegensatz zum neoklassischen Modell wird Migration im sog. HARRIS-TODARO Modell nicht über effektive sondern erwartete Lohn- bzw. Einkommensdifferentiale erklärt.

In der Literatur finden sich verschiedene Variationen des HARRIS-TODARO Modells. BHAGWATI/SRINIVASAN (1974) erweiterten das Modell um Lohn- und Produktionssubventionen. CORDON/FINDLAY (1975) berücksichtigten Kapitalmobilität zwischen städtischen und ländlichen Regionen. FIELDS (1975) betrachtete Mengen- anstelle von Preisanpassungen für den Faktor Arbeit in der städtischen Region. STIGLITZ (1974) analysierte Land-Stadt-Migration im Rahmen eines Modells mit endogener Lohndetermination und CALVO (1978) führte Gewerkschaften im städtischen Arbeitsmarkt ein. SCHMIDT/STILZ/ZIMMERMANN (1994) untersuchten schliesslich, wie der Prozess der Lohn- und Beschäftigungsdetermination im Einwanderungsland durch Gewerkschaften beeinflusst wird.

WEBER (1993). 
Der abdiskontierte Netto-Gegenwartswert der Migration hängt erstens von der Nutzen- bzw. Einkommensdifferenz zwischen dem Ein- und Auswanderungsland $a b\left(Y_{t}^{D}-Y_{t}{ }^{O}\right)$, wobei $P_{t}$ für die Wahrscheinlichkeit steht, dass ein Wirtschaftssubjekt von diesem Nutzen bzw. Einkommen im Zielland überhaupt profitieren kann. Diese Erträge umfassen den Nutzen aus privaten und öffentlichen Gütern. Zweitens hängt der Gegenwartswert von den Transaktionskosten ab. Die Migrationskosten beinhalten in analoger Weise nicht nur monetäre Kosten wie die Transportkosten, die Unterschiede in den Lebenshaltungskosten oder das entgangene Einkommen während der Wanderungsperiode, sondern auch die psychologischen Kosten aufgrund der Trennung von der gewohnten Umgebung mit Familie und Freunden. Drittens spiegelt der Diskontfaktor $r$ die individuelle Gewichtung der Gegenwart gegenüber der Zukunft wider. Letztlich ist auch die Zeitdauer, während der dieser Nutzenstrom anfällt, von Bedeutung. Wichtig ist in diesem Zusammenhang schliesslich die Feststellung, dass jedes Wirtschaftssubjekt, in Abhängigkeit von persönlichen Merkmalen wie dem Alter, dem Geschlecht, der Ausbildung, der Zeitpräferenz sowie der Risikobereitschaft, die Erträge und Kosten unterschiedlich bewerten kann.

Aus dem Humankapitalansatz ergeben sich verschiedene Schlussfolgerungen hinsichtlich der sozio-demographischen und sozio-ökonomischen Merkmale der Einwanderer. ${ }^{66}$ Aufgrund des Investitionscharakters der Migration, bestehen vor allem für jüngere Wirtschaftssubjekte Migrationsanreize, da für sie die Erträge über einen längeren Zeitraum anfallen und damit der Migrationsgewinn grösser ist. Es ist generell zu erwarten, dass diejenigen Komponenten der Migrationsgleichung, die einen positiven Einfluss auf den Netto-Gegenwartswert besitzen, bei den Zuwanderern ausgeprägter sein werden. Sie besitzen demnach höhere Einkommenserwartungen, ausgeprägtere Präferenzen für die Zukunft sowie eine höhere Erfolgswahrscheinlichkeit im Ausland als jene die nicht auswandern. Ferner ist anzunehmen, dass besser Ausgebildete ein geringeres Migrationsrisiko tragen, weil sie besser in der Lage sein sollten, Informationen über die potentiellen Zielländer zu sammeln und zu verarbeiten. Analog zum neoklassischen Ansatz lässt sich abschliessend festhalten, dass die Migrationswahrscheinlichkeit umso höher ist, je grösser die internationalen Lohndifferentiale und je geringer die Migrationskosten sind.

Der zentrale Beitrag des Humankapitalansatzes besteht darin, dass er nicht nur die Bedeutung aggregierter Arbeitsmarktvariablen wie den Lohn- und Arbeitslosigkeitsdifferentialen für die Migrationsentscheidung hervorhebt, sondern dass er die Notwendigkeit unterstreicht, der Heterogenität der Wirtschaftssubjekte bei der Migrationsentscheidung Rechnung zu tragen. Im Rahmen des Humankapitalansatzes ist im Gegensatz zum neoklassischen Ansatz davon auszugehen, dass sich die

66 BAUER/ZIMMERMANN (1995b). 
Wirtschaftssubjekte innerhalb eines Landes hinsichtlich ihrer Migrationsneigungen unterscheiden, weil für sie die Ertragsraten auf spezifischem Humankapital zwischen dem In- und Ausland divergieren können. ${ }^{67}$

Die Tatsache, dass auch der Humankapitalansatz keine umfassende und befriedigende Erklärung dafür zu geben vermag, warum die weiterhin beträchtlichen internationalen Lohndifferentiale keine grösseren Migrationsbewegungen auslösen, hat dazu geführt, dass Unsicherheit bzw. unvollständige Information verstärkt berücksichtigt und explizit in die Modellierung der Migrationsentscheidung miteinbezogen worden ist. ${ }^{68}$ Die Tatsache, dass Migranten nur unvollständige Informationen über ihre Zukunftsperspektiven im Zielland besitzen, hat sowohl grossen Einfluss auf den Umfang als auch auf die Zusammensetzung der effektiv stattfindenden Migrationsbewegungen.

In einer Welt unvollständiger Information kann es für Wirtschaftssubjekte rational sein, die Migrationsentscheidung zu verschieben, um zusätzliche Informationen über die tatsächlichen Kosten und Erträge der Migration zu erlangen. Diese Option besitzt für den potentiellen Migranten einen positiven Wert, ähnlich einer Option auf Finanzmarktinstrumente, und muss daher bei der Modellierung des Migrationsverhaltens mitberücksichtigt werden. Dieser Ansatz wurde ursprünglich entwickelt, um ein besseres Verständnis des Investitionsverhaltens von Unternehmen zu ermöglichen. ${ }^{69}$ Er wurde von BURDA (1995) erstmals in die Migrationsforschung eingeführt, um die trägen Wanderungsbewegungen zwischen Ost- und Westdeutschland zu erklären. BURDA kommt in diesem Modell zum Ergebnis, dass es aufgrund der Unsicherheit über die Lohndifferentiale zwischen zwei Ländern für Wirtschaftssubjekte rational sein kann, sogar in einer Situation, in der die erwarteten Migrationserträge grösser sind als die Migrationskosten, den Migrationszeitpunkt zu verschieben und auf neue Informationen zu warten. Das Modell liefert damit eine theoretische Erklärung für die simultane Existenz grosser Lohnunterschiede und geringer Migrationsbewegungen.

67 LUNDBORG (1991) kommt für Schweden zum Ergebnis, dass im Migrationsverhalten innerhalb des gemeinsamen nordischen Arbeitsmarktes zwar Unterschiede bestehen, dass die Migrationselastizitäten zwischen Dänen, Finnen und Norwegern jedoch nur geringfügig differieren. Unterschiede in den Arbeitslosigkeits- und Lohnniveaus stellen demnach gemäss LUNDBORG die zentralen Erklärungsgrössen für die Zuwanderung nach Schweden dar.

68 Die Bedeutung unvollständiger Information hat erst seit den 80er Jahren in der Migrationsforschung Berücksichtigung gefunden. Das Interesse richtet sich dabei mehr auf den Einfluss asymmetrischer denn unvollständiger Information auf die Migrationsentscheidung [siehe KATZ/STARK (1987), KWOK/LELAND (1984) sowie STARK (1984b)]. Suchtheorien stellen einen zweiten Ansatz zur Modellierung des Einflusses unvollständiger Information auf die Migrationsentscheidung dar. Ein Überblick findet sich in SCHAEFFER (1985).

Eine Übersicht hierzu findet sich in DIXIT/PINDYCK (1994). 
Im Gegensatz zu BURDA modelliert BAUER (1995) den Faktor Unsicherheit in seinem dynamischen Humankapitalansatz nicht auf der Ertragsseite, sondern auf der Kostenseite. Mehrere Argumente sprechen für die Annahme, dass die Kosten der Migration mit grösserer Unsicherheit behaftet sind als die Erträge. Erstens ist internationale Migration, im Gegensatz zu Wanderungsbewegungen innerhalb eines Landes, oft nur dann möglich, wenn Auswanderer bereits eine Arbeitsstelle im Zielland besitzen oder nachweisen können, dass sie für ihren Lebensunterhalt selbst aufkommen können. Zweitens lassen sich die Erträge der Migration einfacher abschätzen, da die Wirtschaftssubjekte lediglich Informationen über die Abgeltung ihrer Fähigkeiten im potentiellen Zielland besitzen müssen. Im Gegensatz dazu orientieren sich die Migrationskosten vor allem an 'weichen' Informationen, wie den Assimilierungskosten im Zielland oder den psychologischen Kosten aufgrund der Trennung von Freunden und Familie, die schwieriger messbar sind.

BAUERS Schlussfolgerungen lassen sich wie folgt zusammenfassen: Erstens muss im Falle von Unsicherheit das Lohndifferential zwischen dem Ein- und Auswanderungsland im Vergleich zum traditionellen Humankapitalansatz grösser sein, um Migrationsbewegungen auszulösen. Dieses Argument kann somit als weiteres Indiz für die Ineffektivität angeführt werden, interregionale Lohndifferentiale durch Faktormobilität auszugleichen. Zweitens liefert der Unsicherheitsansatz von BAUER ein ökonomisches Modell zur Erklärung von Wanderungsbewegungen innerhalb ethnischer Netzwerke. Eine wichtige Aussage dieses Ansatzes besteht darin, dass sich über soziale bzw. ethnische Netzwerke die Kosten und Risiken der Migration reduzieren lassen (siehe auch Abschnitt 4.4). Drittens impliziert die Berücksichtigung von Rückwanderungen, dass das Lohndifferential im Falle von Unsicherheit nicht nur grösser sein muss als im Falle ohne Unsicherheit, sondern auch, dass die Assimilierungskosten steigen müssen, damit Rückwanderungen überhaupt stattfinden. ${ }^{70}$

\subsection{Der AnSATZ DER FAMILIENMIGRATION}

Die bisherigen Ausführungen basierten auf der Annahme, dass Migration ein individuelles Entscheidungsfindungsproblem darstellt. In den letzten Jahren sind jedoch vermehrt Ansätze entwickelt worden, die Migration nicht mehr als individuelle, sondern als kollektive Entscheidung einer Familie oder eines Haushalts interpretieren. Die Migrationsentscheidung im Rahmen dieser 'Neuen Migrationstheorie' kann daher als "... intragroup interaction within a co-operative-game framework ..."71 verstanden werden. ${ }^{72}$

70 Siehe auch STEINER/VELLING (1992). Die Thematik temporärer versus permanenter Migration wird in Kapitel 5 ausführlich behandelt.

71 StRAubHAAR (1988: 78), siehe auch MiNCER (1978) und STARK (1983).

72 Siehe STARK/BLOOM (1985) sowie STARK (1991). 
MINCER (1978) hat in diesem Zusammenhang den Einfluss erhöhter Erwerbsquoten von Frauen auf die Migrationsentscheidung von Familien untersucht. Er gelangte dabei zur Schlussfolgerung, dass die Haushaltsgrösse sowie die Zahl der erwerbstätigen Familienmitglieder die Kosten sowie den Nutzen der Migration beeinflussen. MINCER konnte zeigen, dass sich diejenigen Familienmitglieder, die nicht aus eigenem Antrieb auswandern, ökonomisch verschlechtern und eine Familie aus diesem Grunde nur dann auswandern wird, wenn andere Familienmitglieder diese Verluste kompensieren können. STRAUBHAAR folgert in diesem Zusammenhang, dass "[w]hether the benefits of migration are used to compensate the family for its losses and whether migration of none, one, several or all family members takes place depends on the co-operative-game framework." 73

Migration lässt sich somit als Instrument zur Risikodiversifikation interpretieren und findet daher aufgrund strategischer Überlegungen statt. Entscheidungsträger ist nicht das Individuum sondern ein Kollektiv, i.d.R. die Familie. Das Ziel dieses Kollektivs ist es, durch eine räumliche Diversifikation der Ressourcen, d.h. der Familienmitglieder, das Risiko auf dem Gesamteinkommen der Familie zu minimieren. ${ }^{74}$ Dies wird erreicht, indem Familienmitglieder auf ausländischen Arbeitsmärkten erwerbstätig sind, deren Löhne und Arbeitsbedingungen negativ mit jenen im Herkunftsland korreliert sind. Im Rahmen dieses Ansatzes lässt sich somit erklären, warum Migration auch dann stattfinden kann, wenn keine Lohndifferentiale bestehen. Es ist im Extremfall sogar denkbar, dass Migration für das Kollektiv vorteilhaft sein kann, wenn ein negatives Lohndifferential zum Herkunftsland besteht.

Ein weiterer Ansatz im Rahmen der Neuen Migrationstheorie basiert auf der Annahme, dass die individuelle bzw. kollektive Migrationsentscheidung nicht nur von absoluten, sondern auch von relativen Einkommensüberlegungen beeinflusst wird. ${ }^{75}$ Gemäss dem sog. 'relative deprivation approach' dient Migration als Instrument zur Verbesserung der relativen Einkommensposition im Verhältnis zu einer bestimmten Referenzgruppe. Die Migrationsentscheidung hängt somit nicht nur von den Einkommensunterschieden zwischen dem Herkunfts- und Zielland $\mathrm{ab}$, sondern auch von der Einkommensverteilung innerhalb des Herkunftslandes.

Zusammenfassend lässt sich festhalten, dass Wanderungsbewegungen im Rahmen der 'Neuen Migrationstheorie' nicht als individuelle, sondern als kollektive Entscheidung zu interpretieren sind, bei denen nicht ausschliesslich die Einkommensmaximierung im Vordergrund steht, sondern auch die Risikominimierung hinsichtlich des Gesamteinkommens. Es muss jedoch betont werden, dass die

\footnotetext{
73 STRAUBHAAR (1988: 80).

74 STARK/LEVHARI (1982).

75 Siehe STARK/BLOOM (1985), STARK/TAYLOR (1988), STARK/YITZHAKI (1988), STARK (1984b).
} 
Aussagen der Neuen Migrationstheorie vor allem für Entwicklungsländer Gültigkeit besitzen, in denen es nicht wie in den Industrieländern möglich ist, ein ausreichendes Familieneinkommen über staatliche Sozialprogramme oder über private Versicherungsmärkte zu gewährleisten.

\subsection{DER ANSATZ DER NETZWERKMIGRATION}

Im Rahmen des Netzwerkansatzes stehen dynamische Aspekte des Migrationsprozesses im Vordergrund. ${ }^{76}$ Migration wird in diesem Kontext als ein sich selbst erhaltender Prozess verstanden, in dem sich die Kosten und Risiken der Migration aufgrund sozialer und informationaler Netzwerke über die Zeit verringern. Fehlende bzw. unvollständige Informationen über den Arbeitsmarkt des Ziellandes implizieren, dass sich die ersten Wirtschaftssubjekte hohen Kosten und Risiken im Migrationsprozess gegenübersehen. Die Akkumulation von ziellandspezifischem Humankapital durch die Zuwanderer sowie der damit verbundene Wissenstransfer ins Herkunftsland, der innerhalb des Netzwerks stattfindet, führen zu einer Verringerung der Kosten bzw. des Risikos der Migration und damit zu einer Erhöhung der Ertragsrate bzw. der Migrationswahrscheinlichkeit. Gemäss PoRTES/BÖRÖCZ (1989) sind nicht individuelle oder kollektive Nutzenüberlegungen für die Wanderungsbereitschaft entscheidend, sondern die Mitgliedschaft in einem Migrationsnetzwerk. ${ }^{77}$

Gegen diesen sich selbsterhaltenden Prozess der Migration lassen sich die wanderungsbedingt steigenden Löhne im Herkunftsland und die fallenden Löhne im Zielland anführen, die eine Reduktion des Migrationsnutzens und damit verbunden der Migrationsanreize zur Folge haben. Der Netzwerkansatz impliziert, im Gegensatz zum neoklassischen Ansatz, eine geringere direkte Korrelation zwischen Faktorpreisunterschieden, Beschäftigungsaussichten und der Migrationsentscheidung, da sich aufgrund der Netzwerkbeziehungen die Kosten und Risiken verringern.

\subsection{FAZIT}

Zusammenfassend lässt sich festhalten, dass eine umfassende Erklärung für das Entstehen von Migration nur durch die Kombination der verschiedenen Ansätze gegeben werden kann. Die Ausführungen dieses Kapitels erheben dabei keinen

76 Siehe Carrington/Detraglache/Vishwanath (1996), Hugo (1981), MasSey (1990a, 1990b), sowie MASSEY/ESPAÑA (1987).

77 Siehe auch BOYD (1989). Empirische Evidenz für die Bedeutung ethnischer Netzwerke liefern BAUER/ZIMMERMANN (1995a) für Deutschland. Die substantielle Zuwanderung seit Beginn der 90er Jahre ist weitgehend auf die Existenz ethnischer Netzwerke zurückzuführen. Bei diesen Einwanderern handelt es sich mehrheitlich um sog. Aussiedler oder Übersiedler, d.h. um aus dem Ausland bzw. Ostdeutschland zugewanderte 'ethnische Deutsche'. 
Anspruch auf Vollständigkeit, sondern dienen der Darstellung der wichtigsten Determinanten der Migration sowie den ihnen zugrundeliegenden Annahmen.

Die wichtigsten Unterscheidungsmerkmale zwischen den verschiedenen Ansätzen beziehen sich auf die Art der Migration, die Struktur des Arbeitsmarktes sowie auf den Grad der Homogenität des Faktors Arbeit. Die Ausführungen dieses Kapitels haben gezeigt, dass zu erwarten ist, dass es sich bei den Zuwanderern i.d.R. um gut qualifizierte und junge Wirtschaftssubjekte handelt. Dies gilt vor allem für jene Zuwanderer, die mit der Zielsetzung der Erwerbstätigkeit einwandern, da für sie die grössten Migrationsanreize bestehen. Diese Aussage lässt sich jedoch nicht für andere Zuwanderergruppen, wie die Zuwanderer im Rahmen der Familien-, Netzwerk- oder auch der Flüchtlingsmigration verallgemeinern.

Aus der Zusammensetzung der Migrationsströme bzw. aufgrund der unterschiedlichen Migrationsmotive lassen sich bereits einige vorläufige Hypothesen hinsichtlich der Arbeitsmarktperformance der Zuwanderer ableiten. So ist z.B. zu vermuten, dass Arbeitsmigranten eine vergleichsweise bessere Performance aufweisen werden, da deren Migrationsentscheidung von einer individuellen Nutzenmaximierungsüberlegung geprägt ist. Eine vertiefte Analyse dieser Zusammenhänge erfolgt im nachfolgenden Kapitel. 


\section{ARBEITSMARKTPERFORMANCE DER EINWANDERER}

Die Ausführungen in Kapitel 4 haben gezeigt, dass verschiedene Faktoren für das Entstehen von Migration von Bedeutung sind, die sowohl den Umfang als auch die Zusammensetzung der Zuwanderung beeinflussen. Im Zentrum des ökonomischen wie politischen Interesses steht dabei einerseits die Frage nach dem Erfolg der Einwanderer auf dem schweizerischen Arbeitsmarkt sowie andererseits nach den Ursachen für Performanceunterschiede zwischen In- und Ausländern. Wie bereits in der Einleitung erwähnt wurde, erfolgt die Messung der Arbeitsmarktperformance in der vorliegenden Studie über die Einkommens- und Beschäftigungsperformance.

Die Migrationsliteratur beschäftigt sich im Rahmen der Analyse der Arbeitsmarktperformance von Einwanderern vorwiegend mit der Gegenüberstellung der Einkommensniveaus in- und ausländischer Erwerbstätiger. Die Einkommensniveaus der inländischen Arbeitskräfte dienen dabei als Referenzgrösse. Um eine umfassende Evaluation der Performance der Einwanderer zu gewährleisten, wird in dieser Studie neben der Einkommensperformance auch die Beschäftigungsperformance untersucht, die sich in analoger Weise mit der Analyse der Erwerbsbeteiligung, der Erwerbslosigkeit, der beruflichen Stellung sowie der Beschäftigung von In- und Ausländern in den verschiedenen Wirtschaftszweigen auseinandersetzt. Da beide Untersuchungsgegenstände auf den gleichen ökonomischen Theorien aufbauen, wird im Rahmen der theoretischen Analyse nur dann explizit zwischen den beiden Ansätzen unterschieden, wenn Divergenzen bestehen. Eine getrennte Analyse der Beschäftigungs- und der Einkommensperformance erfolgt im empirischen Teil dieser Studie.

Die ökonomische Forschung zur Arbeitsmarktperformance von Einwanderern basiert vorwiegend auf den Erkenntnissen der Humankapitaltheorie. ${ }^{78}$ Die wichtigsten Aussagen dieses Ansatzes lassen sich wie folgt zusammenfassen: Erstens bestehen vor allem für junge und gut ausgebildete Arbeitskräfte Migrationsanreize, wie die Analyse in Abschnitt 4.2 gezeigt hat. Zweitens ist davon auszugehen, dass sich die migrationsbedingt schlechtere anfängliche Performance der ausländischen Arbeitskräfte, die auf Schwierigkeiten bei der Übertragung bzw. Umsetzung der eigenen Fähigkeiten im Zielland zurückzuführen ist, nach und nach verringern und an jene der Inländer angleichen oder sie sogar übertreffen wird.

Der Erfolg bzw. die Performance der Einwanderer auf dem Arbeitsmarkt des Ziellandes wird im wesentlichen von fünf Faktoren bestimmt, die in diesem sowie

78 Siehe Abschnitt 4.2. Eine kritische Diskussion der Humankapitaltheorie sowie eine Synthese der verschiedenen Ansätze der Humankapitaltheorie findet sich in HENDRICKS (1997). 
im nächsten Kapitel ausführlich diskutiert werden. Erstens kommt den im Herkunftsland erworbenen Fähigkeiten und Qualifikationen zentrale Bedeutung zu. Je besser qualifiziert ein Einwanderer ist, desto einfacher wird es für ihn sein, einen guten Arbeitsplatz mit einem angemessenen Einkommen zu finden. Es gilt daher die Frage zu klären, welche Art der Selektion der Einwanderer stattfindet. Zweitens spielt die internationale Transferierbarkeit des Humankapitals der Einwanderer eine wichtige Rolle. Je besser das erworbene Humankapital international übertragbar ist, desto grösser ist die Wahrscheinlichkeit, dass ein Einwanderer im Zielland erfolgreich sein wird.

Drittens ist der Umfang der von den Zuwanderern im Zielland getätigten landesspezifischen Humankapitalinvestitionen wichtig. Der Umfang dieser Investitionen besitzt für die intertemporale Entwicklung der Arbeitsmarktperformance der Einwanderer grosse Bedeutung. Viertens haben verschiedene intervenierende Faktoren, wie die Nachfrage nach Einwanderern oder die rechtlichen Rahmenbedingungen der Migrationspolitik, grossen Einfluss auf die Performance der Einwanderer. Fünftens hängt die Arbeitsmarktperformance der Einwanderer auch davon $a b$, ob diese die gleichen Chancen und Möglichkeiten auf dem Arbeitsmarkt des Ziellandes besitzen wie die Inländer. Es geht also um die Frage, ob eine Diskriminierung der Einwanderer auf dem Arbeitsmarkt des Ziellandes stattfindet. ${ }^{79}$ Graphisch lassen sich diese Zusammenhänge wie folgt darstellen.

Abb. 5.1: Bestimmungsfaktoren der Arbeitsmarktperformance der Einwanderer

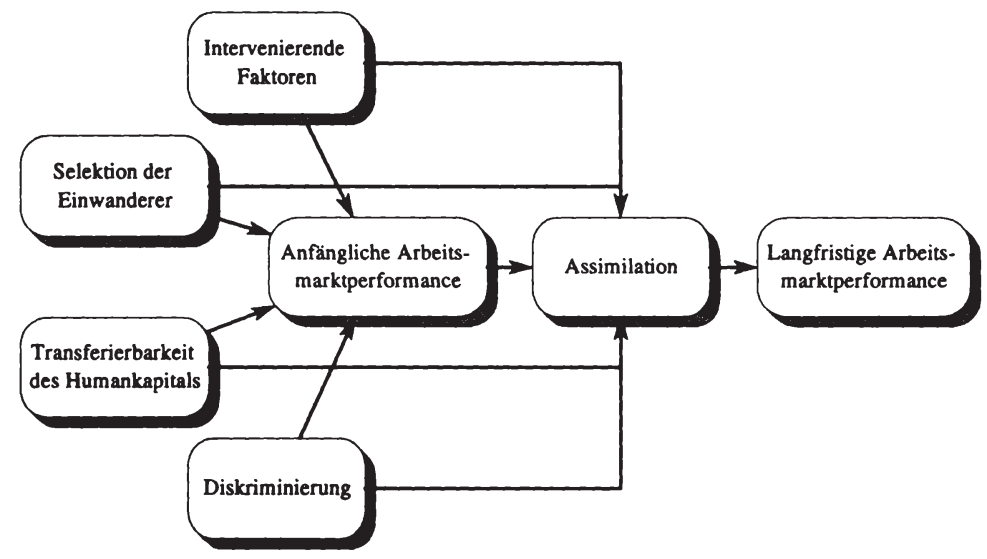

Quelle: Eigene Darstellung

79 Auf diesen Aspekt wird in Kapitel 6 ausführlich eingegangen. 
Das Ziel dieses sowie des nachfolgenden Kapitels ist es, die zentralen Determinanten der Arbeitsmarktperformance zu diskutieren und darauf aufbauend Hypothesen zur Performance der ausländischen Arbeitskräfte auf dem schweizerischen Arbeitsmarkt zu formulieren.

\subsection{SELEKTIVITÄT DER EINWANDERUNG}

Wie bereits zu Beginn dieses Kapitels erwähnt wurde, spielt die Zeitdimension bei der Betrachtung und Bewertung der Arbeitsmarktperformance der Einwanderer eine zentrale Rolle. In diesem und im folgenden Abschnitt erfolgt eine statische Analyse der Arbeitsmarktperformance der Einwanderer. Eine dynamische Betrachtungsweise, welche die intertemporale bzw. längerfristige Entwicklung der Arbeitsmarktperformance der Einwanderer berücksichtigt, folgt in Abschnitt 5.3.

Die ökonomische Analyse der Arbeitsmarktperformance von Einwanderern basiert i.d.R. auf zwei komplementären Ansätzen, die von CHISWICK (1978) in die Migrationsforschung eingeführt wurden. Es handelt sich dabei einerseits um den Ansatz der selektiven Einwanderung, der im Zentrum der Analyse dieses Abschnitts steht, und andererseits um den Ansatz der internationalen Transferierbarkeit von Humankapital, der Gegenstand der Analyse von Abschnitt 5.1.2 ist.

\subsubsection{BESTIMMUNGSFAKTOREN FÜR DIE SELEKTIVITÄT DER EINWANDERUNG}

Es wird i.a. davon ausgegangen, dass Zuwanderer keine zufällige Auswahl aus der Gesamtbevölkerung des Herkunftslandes darstellen. Die Tatsache, dass sich gewisse Wirtschaftssubjekte dafür entscheiden auszuwandern, während andere im Ursprungsland bleiben, impliziert, dass sich Migranten signifikant vom Rest der Bevölkerung unterscheiden. ${ }^{80}$ Verhalten sich Wirtschaftssubjekte gemäss dem in Abschnitt 4.2 diskutierten Humankapitalansatz, so kann von einer positiven Selektion der Auswanderer aus der Gesamtbevölkerung des Herkunftslandes ausgegangen werden, d.h. sie besitzen im Vergleich zum Rest der Bevölkerung des Herkunftslandes ein überdurchschnittlich gutes sozio-ökonomisches Profil. CHISWICK verweist in diesem Zusammenhang darauf, dass in der historischen Literatur Einwanderer generell als "... more able, ambitious and entrepreneurial ..."81 beschrieben wurden. Verschiedene empirische Studien haben ebenfalls gezeigt,

80 VIJVERBERG (1993) beschäftigt sich in diesem Zusammenhang mit der Frage, ob Migranten produktiver sind als jene, die nicht auswandern. Er kommt dabei zum Ergebnis, dass nur dann die produktiveren Wirtschaftssubjekte auswandern, wenn eine hinreichend grosse Korrelation zwischen der personenspezifischen Produktivitätskomponente im Herkunfts- und Zielland besteht. Auf diese Aspekte wird in Abschnitt 5.1.2 ausführlicher eingegangen.

CHISWICK (1994: 97). 
dass Zuwanderer i.d.R. eine bessere Ausbildung besitzen und unternehmerischer veranlagt sind als die im Herkunftsland Verbleibenden. ${ }^{82}$

Wird Migration als Humankapitalinvestition im Rahmen von Kosten-NutzenÜberlegungen verstanden, hat CHISWICK $(1978,1994)$ in einem einfachen Modell gezeigt, dass eine positive Selektion der Einwanderer resultiert. Dieses Ergebnis basiert auf zwei Annahmen. Erstens wird unterstellt, dass das Verhältnis der Erträge auf Humankapital zwischen Hoch- und Niedrigqualifizierten international nicht divergiert, und zweitens, dass sich der Nutzen proportional zum Einkommen entwickelt, die Kosten jedoch teilweise fix sind, so dass die Erträge mit steigendem Einkommen stärker zunehmen. Insofern ist Migration für hochqualifizierte Wirtschaftssubjekte mit einem grösseren absoluten Einkommenszuwachs verbunden als für niedrig Qualifizierte. Der Terminus Humankapital umfasst dabei sowohl beobachtbare als auch nicht-beobachtbare individuelle Charakteristika wie Ausbildung, Motivation oder Ehrgeiz ${ }^{83}$ Algebraisch lassen sich diese Zusammenhänge wie folgt darstellen: ${ }^{84}$

$$
\begin{aligned}
& R_{1}=\frac{W_{1, d}-W_{l, o}}{C_{l, o}+C_{d}} \\
& R_{h}=\frac{W_{h, d}-W_{h, o}}{C_{h, o}+C_{d}} .
\end{aligned}
$$

Gl. (5.1) lässt sich wie folgt interpretieren. $R_{l}$ ist die Ertragsrate auf der Migration für niedrigqualifizierte Arbeitskräfte. $W_{1, o} b z w . W_{l, d}$ stehen für die Einkommen im Herkunfts- und Zielland für niedrigqualifizierte Arbeitskräfte. $\mathrm{C}_{\mathrm{l}, \mathrm{o}}$ entspricht dem entgangenen Einkommen, d.h. dem Produkt aus dem Einkommen im Herkunftsland und der Migrationsdauer. $\mathrm{C}_{\mathrm{d}}$ umfasst schliesslich die direkten, einkommensunabhängigen Kosten. Analoges gilt in Gl. (5.2) für hochqualifizierte Arbeitskräfte. Wird zur Vereinfachung unterstellt, dass die Löhne von Hochqualifizierten in beiden Ländern um k Prozent über jenen der gering Qualifizierten liegen, dann lassen sich Gl. (5.1) und (5.2) wie folgt zusammenfassen:

$$
\mathrm{R}_{\mathrm{h}}=\frac{(1+\mathrm{k})\left\{\mathrm{W}_{\mathrm{l}, \mathrm{d}}-\mathrm{W}_{1,0}\right\}}{(1+\mathrm{k}) \mathrm{C}_{\mathrm{l}, \mathrm{o}}+\mathrm{C}_{\mathrm{d}}}=\frac{\mathrm{W}_{1, \mathrm{~d}}-\mathrm{W}_{\mathrm{l}, \mathrm{o}}}{\mathrm{C}_{\mathrm{l}, \mathrm{o}}+\left\{\mathrm{C}_{\mathrm{d}} / 1+\mathrm{k}\right\}}>R_{l} .
$$

82 Siehe Cross/SANDOS (1981), DaVanzo (1976), SChwartZ (1976) und TIDWICK (1976).

83 CHISwICK (1994) unterstellt in diesem Zusammenhang, dass Zuwanderer vor allem sog. 'allocative skills' besitzen, wie z.B. Entscheidungsfreudigkeit, und weniger sog. 'worker skills', d.h. Fähigkeiten, die direkt mit einer spezifischen Arbeitsstelle verbunden sind. Während die allocative skills im Zielland unmittelbar genutzt werden können, lassen sich hinsichtlich der worker skills keine eindeutigen Aussagen machen [siehe auch SCHULTZ (1975)]. Eine ausführlichere Diskussion dieser Zusammenhänge findet sich im nachfolgenden Abschnitt.

CHISWICK (1994). 
Da der Ertrag für hoch qualifizierte Migranten um $\mathrm{k}$ Prozent höher ausfällt als jener für gering Qualifizierte, die Kostendifferenz jedoch weniger als k Prozent beträgt, weil $C_{d}>0$ ist, bestehen für Höherqualifizierte aufgrund des höheren Ertragspotentials grössere Migrationsanreize. Wird darüber hinaus unterstellt, dass diese besser in der Lage sind, Informationen über das Zielland zu sammeln, dann verringern sich ihre wanderungsbedingten Kosten bzw. ihre direkten Migrationskosten, so dass sich die Ertragsrate für Hochqualifizierte zusätzlich erhöht.

\subsubsection{BeDEUTUNG VON UNTERSCHIEDEN ZWISCHEN HERKUNFTS- UND ZIELLAND}

Diese theoretischen Überlegungen sind in den vergangenen Jahren vor allem von BORJAS $(1985,1987,1989 b, 1991 b, 1994 b)$ kritisiert worden, insbesondere die Symmetrieannahme zwischen Herkunfts- und Zielland. BORJAS zeigte, dass die Erweiterung des ursprünglichen Migrationsmodells um international unterschiedliche Einkommensverteilungen zu weniger klaren Ergebnissen hinsichtlich der Selektion der Einwanderer führt. Die Berücksichtigung differierender Einkommensverteilungen erweist sich deshalb als wichtig, weil sie Hinweise darauf zu geben vermag, wie Humankapital bzw. individuelle Fähigkeiten international abgegolten werden. ${ }^{85}$

Auf der Grundlage des Modells von ROY (1951) unterscheidet BORJAS in seiner Analyse explizit zwischen den beobachtbaren und den nicht-beobachtbaren Komponenten der Humankapitalausstattung, welche das Einkommensniveau bestimmen. Algebraisch lässt sich dies wie folgt darstellen: ${ }^{86}$

$$
\begin{aligned}
& \log w_{0}=X \delta_{0}+\varepsilon_{0} \\
& \log w_{1}=X \delta_{1}+\varepsilon_{1}
\end{aligned}
$$

wobei $w_{0}$ und $w_{1}$ für die jeweiligen Einkommen der Wirtschaftssubjekte im Ausbzw. Einwanderungsland stehen. $\mathrm{X}$ ist ein Vektor, der die beobachtbaren sozioökonomischen Charakteristika wie die Ausbildung und das Alter umfasst. $\delta_{0}$ und $\delta_{1}$ sind Parameter, welche die Entgeltung der sozio-ökonomischen Charakteristika der Wirtschaftssubjekte im Aus- bzw. Einwanderungsland widerspiegeln. $\varepsilon_{0}$ und $\varepsilon_{1}$ bilden schliesslich jene Komponenten des Einkommens ab, die mit den nichtbeobachtbaren Charakteristika der Wirtschaftssubjekte zusammenhängen. Es wird dabei unterstellt, dass $\varepsilon_{0}$ und $\varepsilon_{1}$ miteinander korreliert sind.

85 Bei der Analyse von Einkommensunterschieden ist es wichtig, Nachsteuereinkommen zu vergleichen, da Länder mit einer relativ egalitären Einkommensverteilung wie z.B. Schweden über nahezu konfiskatorische Steuersätze verfügen und die Einkommensverteilung somit aufgrund eines stark progressiven Steuersystems effektiv noch egalitärer wird.

Eine ausführlichere und formalere Darstellung des Modells findet sich in BORJAS (1989b). 
Auf der Grundlage der Gl. (5.4) und (5.5) lassen sich folgende Aussagen hinsichtlich des Umfangs bzw. der Zusammensetzung der Migrationsbewegungen ableiten, welche die Ergebnisse des Grundmodells von CHISWICK bestätigen:

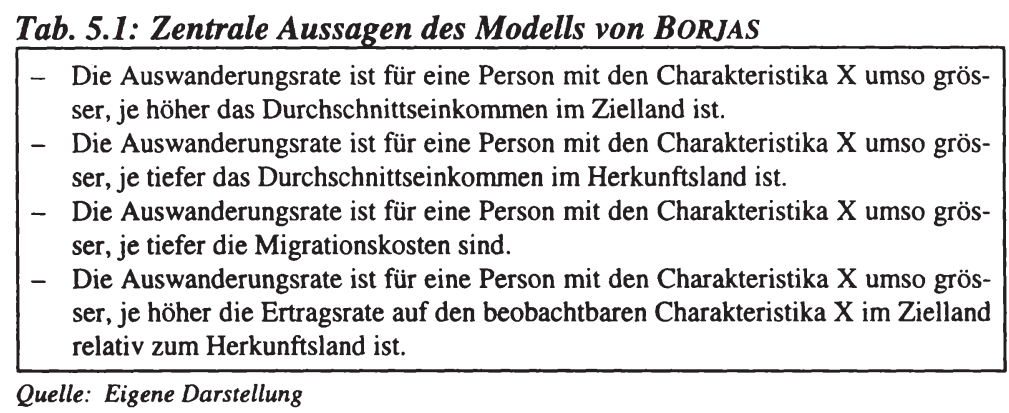

Welche Aussagen lassen sich aus dem Modell von BoRJAs hinsichtlich der Selektion der beobachtbaren und nicht-beobachtbaren Charakteristika der Zuwanderer ableiten? Eine positive Selektion hinsichtlich der beobachtbaren Charakteristika findet dann statt, wenn das Humankapital bzw. die Fähigkeiten der Einwanderer im Zielland mit einem höheren Preis abgegolten werden als im Herkunftsland, d.h. wenn $\left(\delta_{1}-\delta_{0}>0\right) .{ }^{87}$ Die Wirtschaftssubjekte werden somit dorthin auswandern, wo sie den höchsten Ertrag auf ihren beobachtbaren Fähigkeiten realisieren können. Die Selektion hinsichtlich der beobachtbaren sozio-ökonomischen Variablen $\mathrm{X}$ hängt somit allein von der Differenz zwischen den beiden Vektoren $\delta_{0}$ und $\delta_{1}$ $\mathrm{ab}$.

Hinsichtlich der nicht-beobachtbaren Charakteristika gelangt BORJAS zur Schlussfolgerung, dass der Dimension der nicht-beobachtbaren Charakteristika ein grosser Erklärungswert bei der empirischen Analyse von Einkommensunterschieden zukommt: "Unobserved differences among individuals either in 'skills', 'ability' or 'luck' dominate earnings distribution data." 88 Unter der Annahme eines konstanten Vektors beobachtbarer Charakteristika X lassen sich gemäss BORJAS (1991b) drei alternative Selektionsszenarien in Abhängigkeit der nicht-beobachtbaren Charakteristika unterscheiden.

Positive Selektion findet statt, wenn Zuwanderer sowohl im Herkunfts- als auch im Zielland überdurchschnittliche Fähigkeiten aufweisen und daher im Vergleich $\mathrm{zu}$ anderen Wirtschaftssubjekten mit den gleichen Charakteristika $\mathrm{X}$ im Herkunfts- und Zielland eine bessere Performance aufweisen. Als Voraussetzung für eine solche positive Selektion der Zuwanderer muss einerseits eine relativ hohe

87 Ist der Klammerterm negativ, so findet hinsichtlich der beobachtbaren Charakteristika eine negative Selektion der Einwanderer statt.

88

BORJAS (1989b: 467). 
Korrelation zwischen $\varepsilon_{0}$ und $\varepsilon_{1}$ bestehen und das Zielland andererseits eine ungleichere Einkommensverteilung aufweisen als das Herkunftsland.

Negative Selektion findet in diesem Modell demgegenüber dann statt, wenn die Korrelation zwischen $\varepsilon_{0}$ und $\varepsilon_{1}$ zwar hinreichend positiv ist, das Herkunftsland jedoch durch eine ungleichere Einkommensverteilung als das Zielland charakterisiert ist. Negative Selektion kann z.B. entstehen, wenn im Zielland aus wirtschaftspolitischen Überlegungen hohe Einkommen stärker besteuert werden und tiefe Einkommen besser abgesichert sind als im Herkunftsland. Dies hat zur Folge, dass Anreize zur Migration vor allem für niedrig Qualifizierte bestehen, da für sie die grössten Migrationsgewinne resultieren.

Nach BORJAS findet sog. 'Refugee Sorting' schliesslich dann statt, wenn lediglich eine geringe oder negative Korrelation zwischen $\varepsilon_{0}$ und $\varepsilon_{1}$ besteht. Dies impliziert, dass die Zuwanderer im Herkunftsland zwar eine unterdurchschnittliche (Einkommens-)Performance aufweisen, dass sie aber die Einheimischen im Zielland übertreffen können. Dieser Fall lässt sich am besten anhand der Länder des ehemaligen Ostblocks illustrieren, wo aufgrund der administrierten egalitären Einkommensverteilungen die individuellen Einkommen nicht die wahren Produktivitäten der einzelnen Wirtschaftssubjekte widerspiegelten. Aufgrund der künstlichen Abwertung des Humankapitals im Herkunftsland ist jedoch zu vermuten, dass diese Wirtschaftssubjekte im Zielland eine vergleichsweise gute Performance realisieren werden. Zwei Punkte gilt es hier allerdings anzumerken. Erstens lassen sich die Aussagen zum 'refugee sorting' nicht für die gesamte Flüchtlingsmigration verallgemeinern. Zweitens ist zu vermuten, dass die Korrelation zwischen $\varepsilon_{0}$ und $\varepsilon_{1}$ wegen der marktwirtschaftlichen Orientierung der meisten Länder positiv ist und gegen eins tendiert. ${ }^{89}$ In diesem Fall hängt die 'Qualität' der Zuwanderer ausschliesslich vom Verhältnis der Varianzen der Einkommensverteilungen zwischen dem Ein- und dem Auswanderungsland ab.

Zusammenfassend lässt sich festhalten, dass "... selection on the basis of unobserved ability depends entirely on the extent of income inequality in the host and the source countries, and in the correlation between earnings in the two countries. Selection on the basis of observed characteristics, on the other hand, depends on the parameters measuring the price attached by each country's labor market to that particular skill." 90 Mit einer positiven Selektion ist somit für Einwanderer aus jenen Herkunftsländern zu rechnen, die eine egalitärere Einkommensverteilung als die jeweiligen Zielländer aufweisen. Von einer negativen Selektion ist demgegenüber für jene Herkunftsländer auszugehen, die eine vergleichsweise ungleichere Einkommensverteilung besitzen. Diese Aussagen sind aber insofern zu relativie-

89 Das Ausmass der Korrelation zwischen zwei Ländern hängt allerdings auch von der wirtschaftlichen und kulturellen Nähe ab.

BORJAS (1989b: 470-471). 
ren, als eine Vielzahl weiterer Einflussfaktoren die Migrationsbewegungen bestimmt.

Neben der Einkommensverteilung besitzen auch die Arbeitslosenquoten einen Einfluss auf die Selektivität der Zuwanderung. DusTMANN (1993) weist in diesem Zusammenhang darauf hin, dass Unterschiede in den Arbeitslosenquoten zwischen Herkunfts- und Zielländern zu einer negativen Selektion der Zuwanderer führen können. Dies lässt sich mit folgendem Beispiel illustrieren. Liegt die Arbeitslosenquote im Auswanderungsland über jener des Ziellandes und wird weiter unterstellt, dass zwischen der Arbeitslosigkeitswahrscheinlichkeit und dem Qualifikationsniveau eine negative Korrelation besteht, dann ist davon auszugehen, dass vor allem für gering qualifizierte Wirtschaftssubjekte Migrationsanreize bestehen. Diese Aussage trifft insbesondere dann zu, wenn die Arbeitslosenquote innerhalb eines bestimmten Qualifikationssegmentes im Zielland unter jener des Herkunftslandes liegt. Einschränkend ist allerdings darauf hinzuweisen, dass diese Ergebnisse von der Nachfrage des Ziellandes nach ausländischen Arbeitskräften beeinflusst wird. Insgesamt lässt sich folgern, dass die Selektion der Zuwanderer massgeblich von der wirtschaftlichen Lage und den Bedingungen auf den Arbeitsmärkten des Herkunfts- und Ziellandes beeinflusst wird. ${ }^{91}$

Abschliessend ist auf die Bedeutung von Informationsasymmetrien für die Selektivität der Zuwanderung hinzuweisen. Insbesondere von STARK (1991) wurden Modelle asymmetrischer Information verwendet, um den Auswahlprozess der Zuwanderung zu analysieren. Aufgrund von Informationsasymmetrien zwischen in- und ausländischen Arbeitgebern bzw. Arbeitnehmern hinsichtlich der wahren Produktivität der ausländischen Arbeitskräfte werden die Arbeitgeber im Zielland bestrebt sein, in einer ersten Phase allen Einwanderern einen Durchschnittslohn zu bezahlen, da sie anfänglich nicht in der Lage sind, die individuellen Produktivitäten der Einwanderer aufzudecken. Analog zur obigen Diskussion kann dies zur Folge haben, dass vorwiegend gering qualifizierte Wirtschaftssubjekte einwandern, da diese einen überdurchschnittlich hohen Ertrag auf ihren Fähigkeiten realisieren können. Erst mit der Reduktion der Informationsasymmetrien im Zeitverlauf wird es zu einer Entlohnung gemäss den individuellen Qualifikationen kommen.

Dieser Effekt asymmetrischer Information darf jedoch nicht überbewertet werden. Erstens besitzen die hoch qualifizierten Einwanderer Anreize, den Arbeitgebern im Zielland ihre wahre Produktivität zu signalisieren. Zweitens bestehen auch für Arbeitgeber Anreize, die wahren Fähigkeiten der Einwanderer aufzudecken, um die einzelnen Arbeitnehmer möglichst effizient einsetzen zu können. Diese An-

91 Die Bedeutung intervenierender Faktoren wie der Nachfrage nach Einwanderern oder der rechtlichen Rahmenbedingungen der Migrationspolitik werden in Abschnitt 5.4 ausführlich diskutiert. 
reize werden allerdings von der geplanten Aufenthaltsdauer der ausländischen Arbeitskräfte beeinflusst (siehe Abschnitt 5.3). Drittens verhindern Informationsflüsse und Lernprozesse, dass Informationsasymmetrien einen nachhaltigen Einfluss auf die Selektivität der Zuwanderung besitzen. ${ }^{92}$

\subsubsection{BEDEUTUNG DER MigRATIONSMOTIVE}

Die bisherigen Ausführungen beschränkten sich auf die Analyse von Arbeitsmigration. Einkommensmaximierung stellt jedoch lediglich ein Migrationsmotiv dar, das nur einen Teil der insgesamt stattfindenden Wanderungsbewegungen erklärt vermag. Bereits in Kapitel 4 wurde auf die Bedeutung anderer Migrationsformen als der Arbeitsmigration hingewiesen. Es ist in diesem Zusammenhang insbesondere auf Familienmigration bzw. Migration innerhalb von ethnischen Netzwerke sowie Migration aus politischen oder ideologischen Motiven zu verweisen, die als Zuwanderungskategorien zunehmend an Bedeutung gewonnen haben, wie aus Kapitel 3 hervorging. In diesen Fällen stehen bei der Migrationsentscheidung nicht notwendigerweise individuelle Überlegungen zur Nutzenmaximierung im Vordergrund, so dass mit einer weniger positiven Selektion der Einwanderer zu rechnen ist.

\subsection{INTERNATIONALE TRANSFERIERBARKEIT VON HUMANKAPITAL}

Aus dem Wissen um eine positive oder negative Selektion der Einwanderer lassen sich jedoch noch keine klaren Aussagen über die Arbeitsmarktperformance der ausländischen Erwerbstätigen ableiten. Wesentlichen Einfluss auf die Performance der Einwanderer besitzt der Grad der Transferierbarkeit des im Herkunftsland akkumulierten Humankapitals, d.h. das Ausmass, in dem sich die im Herkunftsland erworbenen Fähigkeiten im Zielland umsetzen lassen.

\subsubsection{BESTIMMUNGSFAKTOREN FÜR DIE TRANSFERIERBARKEIT VON HUMANKAPITAL}

Neben der Selektivität der Zuwanderung stellt das Ausmass der Transferierbarkeit des Humankapitals das zweite zentrale Element im humankapitaltheoretischen Modell von CHISWICK (1978) dar. Anhand dieser beiden Konzepte lassen sich gemäss CHISWICK die stilisierten Fakten für die Vereinigten Staaten erklären, dass nämlich ausländische Erwerbstätige in einer ersten Phase nach der Einwanderung als Folge ihrer gesunkenen Produktivität eine schlechtere (Einkommens-)Performance als die Einheimischen aufweisen, dass dieser Einkommensnachteil jedoch mit der Zeit verschwindet und gewisse Einwanderungsgruppen nach einer Zeit-

92 Siehe ChisWick (1988), CoRnelus (1976), MASSEY ET AL. (1987) sowie NORTH/HOUSTON (1976). 
spanne von 10-15 Jahren sogar eine bessere Performance, d.h. einen Einkommensvorsprung, im Vergleich zu den Einheimischen erzielen.

Der anfängliche Einkommensrückgang lässt sich mit der unvollständigen Übertragbarkeit des Humankapitals vom Herkunfts- ins Zielland erklären, da sich die im Herkunftsland erworbenen Fähigkeiten wie die Ausbildung oder die Arbeitserfahrung nur teilweise im Zielland nutzen lassen. ${ }^{93}$ Die herkunftslandspezifischen Elemente der Humankapitalausstattung verlieren daher mit der Auswanderung zumindest teilweise ihren Wert. Der Einkommensrückgang kann auch durch die in Abschnitt 5.1.2 erwähnten Informationsasymmetrien hinsichtlich der effektiven Produktivität der ausländischen Arbeitnehmer bedingt sein. Die intertemporale Verbesserung der Performance der ausländischen Erwerbstätigen hängt demgegenüber mit der positiven Selektion der Einwanderer zusammen.

Der Begriff des landesspezifischen Humankapitals bezieht sich auf Wissen und Fähigkeiten, welche die Grenzproduktivität der Arbeitnehmer in einem bestimmten Land erhöhen, nicht jedoch in einem anderen Land. Als Beispiele hierfür lassen sich Sprachkenntnisse oder die Vertrautheit mit Eigenheiten lokaler Märkte anführen, welche Arbeitnehmer marginal produktiver machen. Verfügen Erwerbstätige vorwiegend über landesspezifisches Humankapital, so können sie im Herkunftsland eine gute Performance erreichen, während sie jedoch in anderen Ländern aufgrund ihrer geringeren Produktivität eine schlechtere Performance erzielen werden. Für Wirtschaftssubjekte mit einem hohen Anteil an landesspezifischem Humankapital fehlen im Rahmen dieses Ansatzes somit weitgehend die Anreize zur Migration. Mehrere Faktoren, die im folgenden diskutiert werden, beeinflussen dabei den Grad der Transferierbarkeit des Humankapitals. ${ }^{94}$

\subsubsection{BEDEUTUNG DER ÄHNLICHKEITEN ZWISCHEN HERKUNFTS- UND ZIELLAND}

Die Transferierbarkeit des Humankapitals hängt vor allem von der Ähnlichkeit zwischen dem Herkunfts- und dem Zielland ab. Der Begriff der 'Ähnlichkeit' bezieht sich hierbei nicht nur auf die Wirtschafts- und Produktionsstruktur, sondern umfasst auch den Grad der geographischen, sprachlichen und kulturellen Nähe zwischen dem Herkunfts- und Zielland. Je mehr die Tätigkeit im Zielland (hinsichtlich des Ablaufs und der Technologie) jener im Herkunftsland entspricht und je besser sich Einwanderer im Zielland kulturell und sprachlich zurechtfinden,

93 STEWART/HYCLAK (1984) weisen in diesem Zusammenhang auf die Bedeutung unterschiedlicher Komponenten des Humankapitals hin. Besteht das Humankapital vorwiegend aus sog. 'general knowledge' ist davon auszugehen, dass sich diese Art des Humankapitals international besser transferieren lässt. Diese Überlegungen decken sich damit mit jenen zu den sog. 'allocative skills' und 'worker skills'.

GREENWOOD/MCDOWELL (1986, 1994). Siehe auch STEINECK (1994). 
aber auch je flexibler sie auf das neue Umfeld reagieren, desto besser werden sie in der Lage sein, ihr Humankapitalpotential im Zielland auszuschöpfen.

Entwicklungsniveau sowie Industrialisierungsgrad des Herkunftslandes im Vergleich zum Zielland können daher als wichtige Determinanten für die Transferierbarkeit des Humankapitals und damit für die Performance der Einwanderer auf dem Arbeitsmarkt des Ziellandes angesehen werden. Findet eine Verlagerung der Zuwanderung in weniger entwickelte oder kulturell und sprachlich weniger verwandte Regionen statt, so ist zu vermuten, dass die Transferierbarkeit des Humankapitals abnehmen wird. Veränderungen in der herkunftslandspezifischen $\mathrm{Zu}$ sammensetzung der Einwanderer kommen somit grosse Bedeutung bei der Beurteilung der Arbeitsmarktperformance der ausländischen Arbeitskräfte zu. BORJAS $(1985,1989 a, 1990,1991 a, 1992 b)$ hat in diesem Zusammenhang für die Vereinigten Staaten nachgewiesen, dass in der Periode von 1940 bis 1980 sowohl eine stetige Verschlechterung der 'skills' als auch der Arbeitsmarktperformance der Einwanderer stattgefunden hat. Er kommt in seiner Analyse dabei zum Schluss, "... that a single factor, the changing national origin mix of the immigrant flow, is almost entirely responsible for this trend." 95

Im Zeitverlauf ist mit steigender Kenntnis der Lebensgewohnheiten, sozialer Normen und arbeitsmarktrelevanter Informationen mit einer Verbesserung der Arbeitsmarktperformance der Einwanderer zu rechnen. Vor allem durch ziellandspezifische Humankapitalinvestitionen im Rahmen der Fort- und Weiterbildung sind Einwanderer in der Lage, ihren Marktwert zu erhöhen. Ausländische Erwerbstätige werden aufgrund ihres Alters in erster Linie über die Vertiefung der Berufserfahrung und weniger über die Akkumulation formaler Schulausbildung versuchen, marktrelevante Qualifikationen zu erwerben. Diese Überlegungen bestätigen somit die Schlussfolgerung, dass ausländische Arbeitnehmer anfänglich eine vergleichsweise schlechte Performance aufweisen, dass jedoch mit zunehmender Aufenthaltsdauer eine kontinuierliche Verbesserung stattfindet. ${ }^{96}$

\subsubsection{BEDEUTUNG DER MigRationSmotive}

Die Transferierbarkeit von Humankapital hängt zudem von den Migrationsmotiven ab. Es ist zu vermuten, dass sich das Humankapital von Arbeitsmigranten im Vergleich zu jenem der Flüchtlings- und Familienmigranten international besser transferieren lässt, da jene diesen Aspekt stärker in ihrer Migrationsentscheidung

95 BORJAS (1992b: 19). BARRETT (1996) konnte jedoch zeigen, dass sich dieser Trend in den 80er Jahren nicht mehr fortgesetzt hat. Auf den Widerspruch zwischen den Ergebnissen von BORJAS und CHISWICK wird im Rahmen der Diskussion der Untersuchungsmethode in Kapitel 8 ausführlich eingegangen.

96 Auf die intertemporale Entwicklung der Arbeitsmarktperformance, die sog. 'Arbeitsmarktassimilation' der Einwanderer wird in Abschnitt 5.3 ausführlich eingegangen. 
bzw. in der Wahl potentieller Zielländer berücksichtigen werden. ${ }^{97}$ Es ist im weiteren davon auszugehen, dass Flüchtlinge ihre Auswanderung in der Regel nicht geplant haben und daher nicht optimal auf die Verhältnisse im Zielland vorbereitet sind. BORJAS (1982) weist in diesem Zusammenhang jedoch darauf hin, dass sich Flüchtlinge auf dem Arbeitsmarkt des Ziellandes schneller assimilieren werden, da für sie die Rückwanderungswahrscheinlichkeit geringer ist und somit grössere Anreize für Investitionen in ziellandspezifisches Humankapital bestehen. Auch JASSO/ROSENZWEIG (1995) und DULEEP/REGETS (1996) kommen in ihren Untersuchungen zum Schluss, dass Familienmigranten zwar anfänglich weniger verdienen als Arbeitsmigranten, dass diese Unterschiede aber mit der Zeit verschwinden.

Es sind im wesentlichen zwei Gründe, die als Erklärung für die Konvergenz der Performance zwischen Arbeits- und Familienmigranten angeführt werden können. Erstens profitieren Einwanderer im Rahmen des Familiennachzugs oder eines ethnischen Netzwerkes von bestehenden Beziehungs- und Informationsnetzwerken, durch die sich die Migrationskosten verringern und die Integration im Zielland erleichtern lässt. DULEEP/REGETS bemerken in diesem Zusamenhang, "... that for immigrants lacking skills that are immediately transferable [...], family and community ties - the development of which would be aided by kinship-based admissions - support immigrants economic adjustment and promote long-term investment". 98 Zweitens besitzt die Nachfrage der inländischen Arbeitgeber nach ausländischen Arbeitnehmern einen wesentlichen Einfluss auf deren Arbeitsmarktperformance. Im Zentrum steht dabei die Frage nach den Branchen und Tätigkeiten, für die ausländische Arbeitskräfte rekrutiert werden. ${ }^{99}$ Analog dazu kann eine Verschlechterung der Performance von Arbeitsmigranten darauf zurückgehen, dass die Verringerung der Migrationskosten die Migration für weniger qualifizierte Personen attraktiver macht.

Ein wesentliches Problem bei der Analyse der internationalen Transferierbarkeit von Humankapital ergibt sich durch die unvollständige internationale Vergleichbarkeit von arbeitsmarktrelevanten Qualifikationen. ${ }^{100}$ Sowohl die Art als auch die Qualität von Ausbildungsgängen und Arbeitserfahrung sind oftmals landesspezifisch und daher nicht oder nur teilweise international vergleichbar. Diesbezügliche Informationsdefizite bestehen sowohl seitens der ausländischen Arbeitnehmer als auch seitens der inländischen Arbeitgeber. Daraus ergeben sich Unsicherheiten hinsichtlich der Übertragbarkeit der im Herkunftsland erworbenen Fähigkeiten

97 Siehe CHISWICK (1980).

98 DULEEP/REGETS (1996: 574).

99 Eine ausführliche Analyse dieses Zusammenhangs erfolgt in Abschnitt 5.4.

100 Für die empirische Analyse stellt sich somit das Problem, wie das im Herkunftsland erworbene Humankapital zu berwerten ist. 
sowie der Anwendbarkeit dieser in den Produktionsprozessen des Ziellandes. ${ }^{101}$ Die Evaluation der tatsächlichen Fähigkeiten von Einwanderern gestaltet sich somit schwierig und beeinträchtigt zumindest in einer ersten Phase die effiziente $\mathrm{Be}$ schäftigung der Einwanderer auf dem Arbeitsmarkt des Ziellandes.

Zusammenfassend lässt sich festhalten, dass der Einfluss von Humankapital auf die Arbeitsmarktperformance der Einwanderer umso positiver ausfallen wird, je besser sich dieses vom Herkunfts- ins Zielland transferieren lässt. CHISWICK (1991) und DusTMANN (1996) haben in diesem Zusammenhang insbesondere auf die Bedeutung von Sprachkenntnissen für die Performance der Einwanderer hingewiesen.

\subsection{ARBEITSMARKTASSIMILATION DER EINWANDERER}

Die bisherigen Ausführungen beruhten auf einem statischen Modell. Es wurde in erster Linie darauf eingegangen, wie sich die Arbeitsmarktperformance der ausländischen Arbeitskräfte unmittelbar nach der Einwanderung darstellt. Von Interesse ist aber vor allem auch die Frage ob, wie und in welchem Ausmass sich die Arbeitsmarktperformance der Einwanderer im Zielland über die Zeit entwickelt, d.h. wie sich die sog. 'Arbeitsmarktassimilation' der Einwanderer darstellt. Es gilt abzuklären, ob die ausländischen Arbeitnehmer ihren anfänglichen Performancerückstand, der sich in tieferen Einkommen bzw. einer schlechteren Beschäftigungssituation äussert, gegenüber den inländischen Erwerbstätigen aufzuholen vermögen. Die vorangegangenen Ausführungen haben bereits gezeigt, dass von einem positiven Zusammenhang zwischen der Performance und der Aufenthaltsdauer der Ausländer auszugehen ist.

\subsubsection{BESTIMMUNGSFAKTOREN FÜR DIE ASSIMILATION DER EINWANDERER}

Auf der Grundlage der theoretischen Überlegungen von CHISWICK (1978) ergibt sich die Schlussfolgerung, dass sich der anfängliche Performancerückstand der Einwanderer im Zeitverlauf reduzieren wird und dass für gewisse Zuwanderergruppen eine Anpassung der Einkommen bzw. der Beschäftigung an das Niveau der inländischen Arbeitnehmer stattfinden wird bzw. gewisse Einwanderergruppen gegenüber den Inländern sogar einen Einkommensvorsprung erreichen werden. ${ }^{102}$ Die Arbeitsmarktassimilierung im Zielland wird neben den sozio-ökono-

101 Siehe WEBER (1993).

102 Eine alternative Erklärung für die gute Performance der ausländischen Arbeitskräfte auf dem Arbeitsmarkt des Ziellandes liefert STARK, der darauf verweist, dass die Performance der Einwanderer nicht nur von sozio-ökonomischen Charakteristika, sondern auch von Anreizen beeinflusst wird: "By shifting the focus of the analysis from the vector of characteristics to the structure of incentives we offer an alternative explanation for the migrants' higher mean income" [STARK (1991: 381)]. Das zentrale Element dieses Ansatzes bildet die Rückwande- 
mischen Charakteristika vom Ausmass der Investitionen in ziellandspezifisches Humankapital (Fort- und Weiterbildung) beeinflusst. Der Zeitdimension kommt somit in zweierlei Hinsicht grosse Bedeutung zu. Erstens erhöht ein längerer Betrachtungszeitraum die Wahrscheinlichkeit, dass der anfängliche Einkommensbzw. Beschäftigungsrückstand wettgemacht werden kann und daher eine Assimilation stattfindet. Zweitens vergrössert eine längere antizipierte Verweildauer die Anreize, in ziellandspezifisches Humankapital zu investieren, da sich die sog. 'pay-off' Periode verlängert.

Vor allem zwei wesentliche Faktoren beeinflussen die Assimilation der ausländischen Arbeitskräfte auf dem Arbeitsmarkt des Ziellandes und damit die Anreize für Einwanderer, in ziellandspezifisches Humankapital zu investieren. Erstens spielt der Zeithorizont des Aufenthalts im Zielland eine wesentliche Rolle, da dieser die Entscheidung zu Investitionen in ziellandspezifisches Humankapital massgeblich beeinflusst. Im Zentrum steht dabei die Frage, ob es sich bei der Zuwanderung um temporäre oder permanente Migration handelt. Zweitens besitzt auch der Zeitpunkt der Einwanderung grosse Bedeutung für die Assimilation. Hier gilt es zu erörtern, ob zwischen den Einwanderern der Ersten und der Zweiten Generation Unterschiede bestehen.

Wie bereits erwähnt wurde, kann die anfänglich schlechtere Performance der ausländischen Arbeitnehmer auch durch Informationsasymmetrien bedingt sein. Auch wenn eine weitgehende Transferierbarkeit des Humankapitals gegeben ist, kann die Tatsache, dass einheimische Arbeitgeber zu wenig über die Qualität dieser Qualifikationen, wissen dazu führen, dass die ausländischen Arbeitskräfte anfänglich lediglich Durchschnittslöhne erzielen und nicht entsprechend ihrer effektiven Produktivität entlohnt werden. Für hochqualifizierte Arbeitnehmer kommt dies einer Verschlechterung der (Einkommens-)Performance gleich. Weisen die Ausländer jedoch trotz vergleichbarer Qualifikationen längerfristig eine schlechtere Performance auf als die Inländer, dann ist von einer Diskriminierung der ausländischen Arbeitskräfte auf dem Arbeitsmarkt des Ziellandes auszugehen. ${ }^{103}$

\subsubsection{BEDEUTUNG DER AUfENTHALTSDAUER IM ZiELLAND}

Die bisherigen Ausführungen basierten auf der Annahme, dass Migration ein einmaliges und permanentes Phänomen darstellt, so dass implizit von Rückwanderungen und damit verbunden temporärer Migration abstrahiert wurde. Werden die Aufenthaltsdauer bzw. der Zeithorizont der Zuwanderung explizit berücksichtigt, stellt sich die Frage nach der Validität der bisherigen Ergebnisse. Die Notwendigkeit dieser Vorgehensweise ergibt sich, weil die Zeitdimension die Anreizstruktu-

rungswahrscheinlichkeit der ausländischen Arbeitskräfte. Siehe auch SCHEAFFER (1995) sowie Abschnitt 5.3.3.

Siehe Kapitel 6. 
ren für Investitionen in ziellandspezifisches Humankapital beeinflusst und daher für temporäre und permanente Zuwanderer mit divergierenden Ergebnissen zu rechnen ist. ${ }^{104}$ Die Rückwanderungswahrscheinlichkeit wird dabei im wesentlichen von drei Faktoren beeinflusst. ${ }^{105}$ Erstens sind die Nähe und die Ähnlichkeit zwischen Herkunfts- und Zielland von Bedeutung. Zweitens spielen die ökonomischen und politischen Bedingungen im Herkunfts- und Zielland eine Rolle. Drittens beeinflusst der Grad der Selektivität die Rückwanderungsentscheidung.

Die Differenzierung der Analyse hinsichtlich temporärer und permanenter Migration ist insbesondere für die vorliegende Studie von Bedeutung, weil die Schweiz seit den 50er Jahren eine Fremdarbeiterpolitik betrieben hat, die von einer hohen Rotation der ausländischen Arbeitskräfte und daher in ihrer Grundkonzeption von temporärer Einwanderung ausging. ${ }^{106}$ Viele dieser 'Gastarbeiter' blieben jedoch langfristig in der Schweiz, wobei die schweizerische Ausländerpolitik der Dauerhaftigkeit der Zuwanderung erst seit den 70er Jahren Rechnung zu tragen begann, indem z.B. der Familiennachzug liberalisiert und die rechtliche Stellung der Familienangehörigen verbessert wurde.

Bestehen zwischen dem Ein- und Auswanderungsland grosse Ähnlichkeiten, dann ist nicht nur mit einem hohen Grad an internationaler Transferierbarkeit herkunftslandspezifischen Humankapitals $\mathrm{zu}$ rechnen, sondern verstärken sich gleichzeitig auch die Anreize für Investitionen in ziellandspezifisches Humankapital, da diese Investitionen auch im Falle einer Rückwanderung Erträge abwerfen werden. Aus diesem Grund ist sowohl mit starken Investitionsanreizen, als auch mit einer relativ hohen Rückwanderungswahrscheinlichkeit zu rechnen. Diese Aussagen besitzen aber auch für den entgegengesetzten Fall signifikanter Unterschiede bzw. grosser räumlicher Distanz zwischen dem Herkunfts- und Zielland Gültigkeit, da die Rückwanderungswahrscheinlichkeit aufgrund mangelnder wirtschaftlicher Perspektiven gering ist.

Im weiteren besitzen auch die Migrationsmotive wesentlichen Einfluss auf die intertemporale Entwicklung der Arbeitsmarktperformance der Einwanderer. Insbesondere Zuwanderer, die aus politischen oder ideologischen Gründen auswandern, haben oft nicht mehr die Möglichkeit, in ihre Heimat zurückzukehren. Da eine Rückwanderung daher unmöglich oder zumindest unwahrscheinlich ist, besteht

104 Siehe DustMaNN (1996).

105 GreENwoOd/MCDowell (1986, 1994). Die Berücksichtigung dieses Zusammenhangs im Rahmen der Analyse der Arbeitsmarktperformance ist deshalb von Bedeutung, weil im Falle selektiver Rückwanderungen mit einer Verzerrung der Performance zu rechnen ist. Findet eine selektive Rückwanderung von ausländischen Wirtschaftssubjekten mit einer unterdurchschnittlichen Performance statt, dann kann die damit einhergehende Verbesserung der Einkommens- und Beschäftigungsperformance der im Zielland verbleibenden ausländischen Arbeitnehmer nicht als verstärkte Arbeitsmarktassimilation interpretiert werden.

Siehe HAMMAR (1985). 
für diese Einwanderer ein grosser Anreiz, sich rasch im Einwanderungsland $\mathrm{zu}$ integrieren und in ziellandspezifisches Humankapital zu investieren. BORJAS kommt daher zum Schluss, dass "... refugees, since they are basically 'stuck' in the host country, have much greater incentives to adapt to the host country than other immigrants who have higher probabilities of return migration". ${ }^{107}$

BORJAS (1990) konnte für die Vereinigten Staaten zeigen, dass das Einkommenswachstum von Flüchtlingen aus Ländern des ehemaligen Ostblocks rund 1 Prozent über jenem der Flüchtlinge aus westeuropäischen Staaten liegt. CHISWICK (1979, 1986, 1994) kam in seiner empirischen Analyse demgegenüber zum Ergebnis, dass der Einkommensrückstand von Flüchtlingen zu Beginn nicht nur grösser ist als für andere Einwanderergruppen, sondern dass diese trotz eines relativ steilen Einkommensprofils nicht das Einkommensniveau von ökonomisch motivierten Zuwanderern erreichen. Die Performance von Flüchtlingen und Asylsuchenden unterliegt somit insofern einem trade-off, als die Investitionsanreize partiell durch eine allfällige negative Selektion dieser Einwanderergruppe aufgewogen werden können.

Schliesslich kommt auch der Selektivität der Zuwanderung bei der Arbeitsmarktassimilation eine grosse Bedeutung zu. Eine positive Selektion impliziert, dass für diese Einwanderer aufgrund des hohen Ertragspotentials grosse Anreize für Investitionen in ziellandspezifisches Humankapital bestehen. Es ist daher zu vermuten, dass dieser Umstand ebenfalls einen negativen Einfluss auf die Rückwanderungswahrscheinlichkeit besitzt.

Zusammenfassend lässt sich somit festhalten, dass für Wirtschaftssubjekte mit einer hohen Rückwanderungswahrscheinlichkeit bzw. temporäre Zuwanderer lediglich geringe Anreize für Investitionen in ziellandspezifisches Humankapital bestehen. Dieses Ergebnis lässt sich auf die kurze pay-off Periode der Investitionen zurückführen. Es liegt daher die Vermutung nahe, dass temporäre Zuwanderer im Vergleich zu permanenten Einwanderern ein flacheres Einkommens- bzw. Beschäftigungsprofil aufweisen. Generell ist die Performance von Einwanderern umso besser, je geringer die Rückwanderungswahrscheinlichkeit für die einzelnen Wirtschaftssubjekte ist und damit verbunden, je grösser die Anreize sind, in landesspezifisches Humankapital zu investieren. ${ }^{108}$

107 BORJAS (1989b: 473).

108 Wie bereits erwähnt, gehen STARK (1991) und SCHAEFFER (1995) davon aus, dass Performanceunterschiede im wesentlichen durch divergierende Anreize und nicht durch Unterschiede in den sozio-ökonomischen Charakteristika der einzelnen Wirtschaftssubjekte bedingt sind. Sie gelangen daher zum Ergebnis, dass eine hohe Rückwanderungswahrscheinlichkeit einen positiven Einfluss auf die Performance der ausländischen Arbeitskräfte besitzt: "The possibility of return [migration] to a low-wage economy results in an intertemporal substitution in the supply of labor. As greater work effort translates into higher income, migrants will outperform comparable native-born workers." [STARK (1991: 389)]. 


\subsubsection{BEDEUTUNG DES EINWANDERUNGSZEITPUNKTES}

Neben der Selektivität der Zuwanderung, der Transferierbarkeit des Humankapitals und der Aufenthaltsdauer, kommt auch dem Einwanderungszeitpunkt wesentliche Bedeutung bei der Erklärung der Arbeitsmarktperformance der Einwanderer im Zielland zu. Im Zentrum der Analyse steht die Frage, ob zwischen Einwanderern der Ersten und Zweiten Generation Performanceunterschiede bestehen. ${ }^{109}$

Diese Vermutung liegt aus mehreren Gründen nahe, wobei davon auszugehen ist, dass die Einwanderer der Zweiten Generation eine bessere Performance aufweisen. Erstens haben Ausländerkinder einen grossen Teil ihrer Ausbildung im Zielland erhalten, so dass sie bereits über einen grossen Bestand an ziellandspezifischem Humankapital verfügen und daher ähnlich qualifiziert sind wie Inländer. Zweitens sind sie im Zielland i.d.R. besser integriert, da sie im dortigen kulturellen und sozialen Umfeld aufgewachsen sind. KossoudJI (1989:520) kommt in diesem Zusammenhang für die Vereinigten Staaten zum Ergebnis, dass "... economic assimilation may not be as much a matter of how long an individual has been in the United States as it is of the timing of migration. Immigrants who arrive before schooling is completed are likely to gain more with experience in the U.S. ...".

CHISWICK (1977) äusserte in diesem Zusammenhang die Vermutung, dass sich die positive Selektion der Eltern über die Erziehung auf deren Kinder überträgt, so dass diese z.B. ebenfalls überdurchschnittlich motiviert sind. CHISWICK fand jedoch für diese Hypothese keine empirische Evidenz. Aus seiner Analyse ergab sich lediglich ein geringfügiger Einkommensvorteil der Einwanderer der Zweiten Generation im Vergleich zu jenen der Ersten Generation. ${ }^{110}$

BORJAS (1992a, 1993, 1994a) ging in seiner Analyse stärker der Frage nach, ob und in welchem Ausmass die Fähigkeiten der Einwanderer einer Generation auf die nächste Generation übertragen werden. Er kam dabei zum Ergebnis, dass zwar über Generationen hinweg eine langsame Arbeitsmarktassimilation der Einwanderer stattfindet, dass jedoch zwischen den Durchschnittseinkommen der Ersten und der Zweiten Generation innerhalb ethnischer Gruppen eine starke Korrelation besteht. Er konnte zeigen, dass sog. ethnisches Kapital, d.h. die Summe der tradierten Fähigkeiten einer Bevölkerungsgruppe mit gleichem kulturellen Hintergrund und gleicher Abstammung, einen persistenten Einfluss auf die Performance besitzt. ${ }^{111}$ Aus diesem Grund gelangt BoRJAS zur Schlussfolgerung, dass "... the

109 Die Einwanderer der Zweiten Generation umfassen jene ausländischen Staatsangehörigen, die entweder im Einwanderungsland geboren wurden oder zumindest einen grossen Teil ihrer Kindheit im Einwanderungsland verbracht haben. In der empirischen Analyse des dritten Teils liegt die Grenze zwischen Erster und Zweiter Generation bei einem Einwanderungsalter von 15 Jahren.

110 Siehe auch CARLINER (1980), der zu ähnlichen Ergebnissen kommt wie CHISWICK.

111 Siehe ZIMMERMANN (1993). 
same source-country characteristics that are such crucial determinants of the labor market experiences of immigrants will influence the experiences of their children". ${ }^{112}$ Politische Massnahmen im Bereich der Ausbildung oder der Selektion der Einwanderer bestimmen damit nicht nur den Erfolg der Einwanderer der Ersten Generation, sondern haben auch grossen Einfluss auf den Erfolg der nachfolgenden Generationen.

Als Fazit der Analyse dieses Abschnitts ergibt sich somit die Feststellung, dass die Einwanderer keine homogene Gruppe bilden und daher substantielle Performanceunterschiede zwischen verschiedenen Einwanderergruppen bestehen können. Der Heterogenität der Einwanderer wird im empirischen Teil dieser Studie Rechnung getragen, indem in der Analyse sowohl zwischen Einwanderungskohorten als auch verschiedenen Nationalitätengruppen unterschieden wird.

\subsection{EINFLUSS INTERVENIERENDER FAKTOREN}

Die Analyse dieses sowie des vorangegangenen Kapitels haben gezeigt, dass eine Vielzahl unterschiedlicher Faktoren die Entstehung und die Zusammensetzung von Migrationsströmen beeinflusst. Es handelt sich dabei erstens um ökonomische Faktoren wie eine bessere Beschäftigungssituation bzw. bessere Arbeitsbedingungen oder höhere Löhne im Zielland, zweitens um demographische Faktoren wie den Umfang und die Altersverteilung der Arbeitsbevölkerung in den Herkunftsländern, sowie drittens um politische Faktoren wie politische Verfolgung oder Repression im Herkunftsland, die zu sog. push-Migration, d.h. angebotsbestimmter Migration führen.

Die Existenz migrationswilliger Wirtschaftssubjekte stellt jedoch, wie bereits erwähnt, lediglich eine notwendige, nicht aber eine hinreichende Bedingung für das Entstehen von Zuwanderung dar, da die effektiv stattfindende Migration sowohl von der Nachfrage der Aufnahmeländer nach ausländischen Arbeitskräften, als auch von den Migrationspolitiken, d.h. den Zuwanderungsbestimmungen der einzelnen Zielländer abhängt. STRAUBHAAR bemerkt in diesem Zusammenhang: "For international migration to occur it is necessary and sufficient that, first, there is a demand for foreign labor in the immigration country, and, second, that there are no immigration restrictions to prevent the immigration of active foreigners." 113 Der Umfang des Arbeitsangebots sowie der Arbeitsnachfrage auf dem internationalen Migrationsmarkt wird schliesslich sowohl vom internationalen Güterhandel als auch von der internationalen Faktormobilität beeinflusst, d.h. dem Grad der Substitutionalität bzw. Komplementarität zwischen Migration und Handel.

112 BORJAS (1993: 133).

113 STRAUBHAAR (1986: 844). 


\subsubsection{NACHFRAGE NACH AUSLÄNDISCHEN ARBEITSKRÄFTEN}

Die Nachfrage nach ausländischen Arbeitskräften ist eine Reaktion auf Knappheiten auf dem heimischen Arbeitsmarkt. Es kann sich dabei um eine gesamtwirtschaftliche Knappheit während einer Boomphase handeln oder auch nur um branchenspezifische Engpässe. Es ist allerdings zu beachten, dass die Nachfragekomponente lediglich jenen Teil der Einwanderung beeinflusst, der die ausländischen Arbeitskräfte, d.h. die Arbeitsmigration, umfasst. Zuwanderung, die aus anderen Motiven wie der Familienzusammenführung oder der Asylsuche erfolgt, lässt sich nicht über die Nachfrageseite steuern, sondern hängt in erster Linie von den $\mathrm{Zu}$ wanderungsbestimmungen der Migrationspolitik ab. ${ }^{114}$

Die Arbeitsnachfrage der Unternehmen ist für die Analyse der Arbeitsmarktperformance der ausländischen Arbeitnehmer von zentraler Bedeutung, da sie entscheidenden Einfluss auf die Zusammensetzung und den Umfang der Zuwanderung ausländischer Arbeitskräfte besitzt. Im speziellen ist erstens von Bedeutung, welche Branchen ausländische Arbeitskräfte für welche Tätigkeiten nachfragen und zweitens, über welchen Zeithorizont sich die Beschäftigung der ausländischen Arbeitskräfte erstreckt. Diese zeitliche Dimension wird stark von den gesetzlichen Bestimmungen beeinflusst, auf die im folgenden Abschnitt ausführlicher eingegangen wird.

Die Nachfrage der verschiedenen Branchen nach ausländischen Arbeitskräften und die dadurch bedingte sektorielle Allokation der Arbeitskräfte ist deshalb von Bedeutung, weil dadurch das Anforderungsprofil und damit verbunden die $\mathrm{Hu}-$ mankapitalausstattung der ausländischen Arbeitskräfte bestimmt bzw. beeinflusst wird. Die sozio-demographischen Profile der Zuwanderer hängen somit entscheidend davon ab, welche Zielsetzungen die einzelnen Unternehmen mit der Rekrutierung ausländischer Arbeitskräfte verfolgen. Die Schweiz kann hier als anschauliches Beispiel dienen, da gleichzeitig sowohl hoch- als auch niedrigqualifizierte Arbeitskräfte rekrutiert werden.

Die Rekrutierung niedrigqualifizierter ausländischer Arbeitskräfte findet statt, weil sich für bestimmte Tätigkeiten entweder keine einheimischen Arbeitskräfte finden lassen oder weil deren Beschäftigung zu teuer wäre. Die in diesen Arbeitsmarktsegmenten tätigen Ausländer sind daher i.d.R. niedrigqualifiziert und üben vorwiegend manuelle Tätigkeiten z.B. in der Verarbeitenden Produktion oder im Baugewerbe aus. Obschon in einer Volkswirtschaft immer ein gewisser Bedarf an unqualifizierten Arbeitskräften besteht, gibt die Beschäftigung kostengünstiger ausländischer Arbeitnehmer vielen inländischen Unternehmen die Möglichkeit, notwendige strukturelle Anpassungen hinauszuzögern. Problematisch wird es,

114 Dieser Aspekt ist insbesondere für die Schweiz von Bedeutung, da in den vergangenen Jahrzehnten die Familien- und Flüchtlingsmigration zunehmend an Gewicht gewonnen haben (siehe Kapitel 3). 
wenn sich diese Strukturreformen nicht mehr aufschieben lassen und daher mit einem substantiellen Stellenabbau zu rechnen ist. Verschärft wird diese Situation dadurch, dass die freigesetzten ausländischen Arbeitnehmer zudem Schwierigkeiten haben werden, aufgrund ihrer unvorteilhaften Qualifikationsprofile eine neue Stelle zu finden.

Auf der anderen Seite rekrutieren Unternehmen hochqualifizierte ausländische Arbeitskräfte, um im intensivierten internationalen Wettbewerb konkurrenzfähig $\mathrm{zu}$ bleiben. Insbesondere in einem Land wie der Schweiz, das über keine natürlichen Ressourcen verfügt, stellt das Humankapital die zentrale Ressource dar. Unternehmen, die vorwiegend qualifizierte Arbeitskräfte einsetzen, sind i.d.R. in zukunftsträchtigen und wachsenden Märkten tätig, so dass ihnen längerfristig eine immer grössere Bedeutung zukommen wird. Die Zukunftsperspektiven und damit die intertemporale Entwicklung der Arbeitsmarktperformance hochqualifizierter Arbeitskräfte sind aus diesem Grunde weitaus positiver zu beurteilen als jene Niedrigqualifizierter. Es ist daher mit beträchtlichen Diskrepanzen hinsichtlich der Einkommen und der Beschäftigung zwischen diesen beiden Gruppen zu rechnen.

Zusammenfassend lässt sich festhalten, dass die Arbeitsnachfrage als eine zentrale Determinante des Umfangs sowie der Zusammensetzung des Zuwanderungsstroms angesehen werden kann. Besteht eine grosse inländische Nachfrage nach unqualifizierten ausländischen Arbeitskräften, so ist mit einer relativ schlechten Performance sowie einem geringen Beitrag dieser Arbeitnehmer zur Prosperität des Ziellandes zu rechnen. Diese Überlegungen implizieren damit aber gleichzeitig, dass Informationen über die Beschäftigungsstruktur bzw. die Verteilung der ausländischen Arbeitskräfte auf die verschiedenen Branchen einen wesentlichen Beitrag zur Erklärung der Performance der Einwanderer zu leisten vermögen. Schliesslich wird die Arbeitsnachfrage auch von der Migrationspolitik beeinflusst. In diesem Zusammenhang ist insbesondere auf die Gastarbeiterpolitik der 50er und 60er Jahre hinzuweisen, welche die Rekrutierung niedrig qualifizierter Arbeitskräfte begünstigte. ${ }^{115}$

Der Zeithorizont der Beschäftigung besitzt ebenfalls einen Einfluss auf die Arbeitsmarktperformance der ausländischen Arbeitskräfte. Erstens vermittelt er einen Eindruck darüber, ob die ausländischen Arbeitskräfte nur zur Überbrückung konjunktureller Engpässe eingesetzt werden, oder ob sie längerfristig zur Vergrösserung des Bestandes der Erwerbsbevölkerung beitragen. Dieser Zusammenhang ist insbesondere für die Schweiz von Bedeutung, die während Jahrzehnten im Rahmen der Gastarbeiterpolitik vor allem daran interessiert war, die zyklischen Schwankungen der Arbeitsnachfrage durch die Beschäftigung ausländischer $\mathrm{Ar}$ beitskräfte zu glätten. ${ }^{116}$ Es ist davon auszugehen, dass Arbeitskräfte, die lediglich

115 Auf diesen Zusammenhang wird im nächsten Abschnitt ausführlich eingegangen.

116 Siehe auch Kapitel 5. 
zur Überbrückung saisonaler Schwankungen rekrutiert werden, ein geringeres Fähigkeitspotential aufweisen als jene, die eine längerfristige Beschäftigung anstreben. ${ }^{117}$ Gleichzeitig besitzt der Zeithorizont der Beschäftigung auch grossen Einfluss auf die Anreize ausländischer Arbeitskräfte in landesspezifisches Humankapital zu investieren.

Im Zusammenhang mit der Nachfrage nach ausländischen Arbeitskräften stellt sich auch die Frage nach der Bedeutung der Angebots- und Nachfrageeffekte, d.h. der 'kurzen Marktseite'. ${ }^{118}$ Die Nachfrage nach qualifizierten Arbeitskräften ist weitgehend nachfragebestimmt, da aufgrund des intensivierten internationalen Wettbewerbsdrucks in westlichen Industrie- und Dienstleistungsgesellschaften wie der Schweiz ein zunehmender Bedarf an hochqualifizierten Arbeitskräften besteht und damit eine internationale Knappheit dieser Ressource besteht. ${ }^{119}$ Die Nachfrage nach unqualifizierten Arbeitskräften ist demgegenüber weitgehend angebotsbestimmt, da für diese Gruppe, deren Angehörige meist aus peripheren Regionen Europas bzw. aus Schwellen- und Entwicklungsländern stammen, ein rückläufiger Bedarf besteht, der sich aus der zunehmenden Rationalisierung bzw. Automatisierung vieler Arbeitsprozesse ergibt. Ökonomische, demographische und politische Faktoren werden aber auch künftig dazu führen, dass ein substantielles Migrationspotential an gering qualifizierten ausländischen Arbeitskräften existieren wird, das die Nachfrage bei weitem übersteigt.

Schliesslich ist auf die Bedeutung der Arbeitsmarktstruktur bei der Analyse der Arbeitsmarktperformance hinzuweisen, insbesondere beim Vergleich der Einkommen von Einwanderern und Einheimischen. GREGORY/ANSTIE/KLUG (1991) weisen z.B. darauf hin, dass vor allem institutionelle, die Arbeitsmarktstruktur betreffende Faktoren dafür verantwortlich sind, dass niedrig qualifizierte Einwanderer in Australien besser bezahlt werden als vergleichbare Einwanderer in den Vereinigten Staaten. Erstens ist in Australien der Grad der gewerkschaftlichen Organisation bedeutend höher als in den Vereinigten Staaten. Zweitens besteht in Australien eine relativ starke Regulierung des Arbeitsmarktes mit Mindestlöhnen. Drittens hat die australische Regierung während langer Zeit hohe Zölle erhoben, um die einheimische Industrieproduktion vor ausländischer Konkurrenz zu schützen und damit für eine künstliche Erhöhung des Lohnniveaus gesorgt. Diese Faktoren haben dazu geführt, dass in Australien eine weniger starke Ungleichverteilung der Einkommen besteht und daher auch niedrig Qualifizierte relativ gut bezahlt werden. Diese Überlegungen verdeutlichen gleichzeitig, dass bei internatio-

117 Es ist hier allerdings darauf hinzuweisen, dass in multinationalen Unternehmen insbesondere im Rahmen der Weiterbildung des Führungsnachwuchses, ein reger, zeitlich beschränkter, internationaler Austausch stattfindet [siehe WOLTER (1996)].

118 Siehe FISCHER/STRAUBHAAR (1996a).

119 Siehe BORJAS (1990). 
nalen Vergleichen der Arbeitsmarktperformance von Einwanderern grosse $\mathrm{Zu}$ rückhaltung geboten ist, da die jeweiligen Ergebnisse stark von landesspezifischen Arbeitsmarktstrukturen geprägt sein können.

\subsubsection{RECHTLICHE RAHMENBEDINGUNGEN DER MIGRATIONSPOLITIK}

Die Migrationspolitik stellt nicht nur einen wichtigen Faktor bei der Erklärung der Entstehung von Migration dar, sondern beeinflusst über die Zulassungsbestimmungen die Zusammensetzung und damit verbunden auch die Selektion der $\mathrm{Zu}$ wanderer. Erst das Zusammenspiel von Migrationsangebot bzw. -nachfrage und den rechtlichen Rahmenbedingungen führt zur Zuwanderung ins Zielland. Die rechtlichen Rahmenbedingungen sind von Bedeutung, weil sie die Zulassungsbedingungen für die Zuwanderung festlegen. Jede Form der Einwanderungsbegrenzung erfordert Zulassungskriterien, die unterschiedlichen Allokationsprinzipien folgen. ${ }^{120}$ Neben egalitären (absoluten Verboten), zeitbezogenen (Wartelisten) oder statusbezogenen Kriterien wie dem Alter, dem Geschlecht oder der Rasse ist insbesondere auf sozio-ökonomische Kriterien wie die Ausbildung oder die $\mathrm{Be}$ rufserfahrung hinzuweisen. ${ }^{121}$ Die gleichzeitige Berücksichtigung verschiedener Faktoren kann dabei explizit, z.B. im Rahmen eines Punktesystems, oder implizit, durch die Einwanderungsbehörden, erfolgen. In der Praxis gelangen i.a. eine Vielzahl verschiedener Zulassungskriterien zur Anwendung.

Diese Überlegungen zeigen, dass die Migrationspolitik bzw. die gesetzlichen Bestimmungen der Ausländerpolitik grossen Einfluss auf die Arbeitsmarktperformance der ausländischen Arbeitskräfte ausüben. Welche Allokationsprinzipien zur Bestimmung der Zulassungskriterien Anwendung finden, hängt dabei im wesentlich von den Zielsetzungen ab, die mit der Zulassungspolitik verfolgt werden. In vielen Ländern ist die Migrationspolitik historisch geprägt, so dass die Zulassungsbedingungen in diesen Ländern statusbezogen ist. Als Beispiele lassen sich Frankreich, Grossbritannien oder die Niederlande anführen, deren Einwanderer während langer Zeit vorwiegend aus den ehemaligen Kolonien stammten. Stehen arbeitsmarktpolitische Überlegungen im Vordergrund, so erscheint eine Orientierung der Migrationspolitik an sozio-ökonomischen Kriterien am effizientesten, wie dies z.B. in Kanada oder Australien praktiziert wird. ${ }^{122}$

Bei der Konzeption und materiellen Ausgestaltung der Migrationspolitik sowie der damit verbundenen Formulierung der Zulassungskriterien kommt ein politökonomisches Element ins Spiel. Interessengruppen werden sich für Zulassungskriterien einsetzen, die ihren spezifischen Bedürfnissen Rechnung tragen. Zwei

120 Für eine Übersicht und Bewertung von Allokationsprinzipien siehe ELSTER (1991).

121 Siehe CHIODI-FERRO (1994).

122 Für eine Analyse und Bewertung unterschiedlicher Migrationspolitiken siehe FASSMANN/MÜNZ (1996), HAMMAR (1985), VELLING/WOYDT (1993), und ZIMMERMANN (1994). 
Faktoren entscheiden dabei über den Einfluss der verschiedenen Interessengruppen. Erstens wird er von der Organisierbarkeit beeinflusst. ${ }^{123}$ Nur gut organisierten Interessengruppen gelingt es, ihren Forderungen politisches Gewicht zu verleihen. In der Schweiz sind es gemäss DHIMA (1991: 151) vor allem Interessengruppen aus zwei Bereichen, die "... den Zustand der Latenz überwinden und zu wirksamen Akteuren im immigrationspolitischen Geschehen avancieren ...". Es handelt sich dabei einerseits um die Gruppe der Arbeitgeber, welche die Einwanderungsrente abzuschöpfen versucht, und andererseits um die Gruppe der arbeitsintensiven Branchen des binnenorientierten, wenig wettbewerbsfähigen Sektors. Aufgrund der geringen Zahl potentieller Mitglieder, des hohen Nutzenzuwachses für den Fall der Zielerreichung sowie der Genauigkeit der Ziele, lassen sich diese beiden Sektoren besonders gut organisieren.

Zweitens hängt die Einflussnahme der Interessengruppen auch vom politischen System ab, in dem sie agieren. Insbesondere hier eröffnet ein direkt-demokratisches und föderalistisches Staatswesen, wie jenes der Schweiz, grosse Einflussmöglichkeiten für die Partikularinteressen von Lobbyisten. ${ }^{124}$ Neben dem Einfluss auf Bundesebene über die direkt-demokratischen Instrumente der Initiative oder des Referendums versuchen die Interessengruppen auch auf kantonaler Ebene den Vollzug der jährlichen Begrenzungsverordnung (BVO) sowie die Verteilung der kantonalen Einwanderungskontingente zu beeinflussen. ${ }^{125}$

Abschliessend gilt es darauf hinzuweisen, dass die Ausländer- bzw. Migrationspolitik den Assimilierungsprozess der ausländischen Arbeitskräfte über die Rechte und, damit verbunden, die wirtschaftlichen Möglichkeiten, die den ausländischen Wirtschaftssubjekten eingeräumt werden, beeinflusst. Je eingeschränkter die Rechte der ausländischen Wirtschaftssubjekte auf dem Arbeitsmarkt des Ziellandes sind, desto eher werden sie eine vergleichsweise schlechte Performance aufweisen. Da sich die Rechtsstellung der Einwanderer im Aufnahmeland mit zunehmender Verweildauer verbessert, ist davon auszugehen, dass über die Zeit eine Verbesserung der Arbeitsmarktperformance der ausländischen Arbeitskräfte stattfinden wird.

\subsubsection{FAKTORMOBILITÄT UND GÜTERHANDEL}

Abschliessend soll der Zusammenhang zwischen Güterhandel und Faktormobilität (Arbeitskräftewanderung und Direktinvestitionen) erörtert werden. Dieser Aspekt ist deshalb von Interesse, weil er sowohl das Angebot als auch die Nachfrage nach ausländischen Arbeitskräften beeinflusst. Im Kern geht es dabei um die Frage der Substitutionalität bzw. Komplementarität von Güterhandel und Faktormobili-

\footnotetext{
123 Siehe OLSON (1965).

124 Siehe DHIMA (1991), BORNER/BRUNETTI/STRAubHAAR $(1990,1994)$.

125 Siehe BORNER/STRAUBHAAR (1995).
} 
tät. Es gilt also zu überprüfen, ob internationale Faktorbewegungen den internationalen Güterhandel verstärken oder verringern.

Aus aussenwirtschaftstheoretischer Sicht lassen sich verschiedene Ursachen für Faktormobilität anführen. ${ }^{126}$ Erstens kann sie als Reaktion auf bestehende Handelshemmnisse sowie zweitens als Folge international nicht-handelbarer Güter interpretiert werden. Drittens kann sie das Resultat international unterschiedlicher Skalenerträge bzw. verschiedenartiger Produktionstechnologien sein, welche die Voraussetzung für internationale Spezialisierung und unvollständige Wettbewerbsmärkte darstellen. Sowohl im ersten als auch im zweiten Fall implizieren internationale Faktorbewegungen eine substitutionale Beziehung zum Güterhandel. Im dritten Fall besteht hingegen eine komplementäre Beziehung zwischen Faktormobilität und internationalem Güterhandel.

Je ähnlicher sich also die einzelnen Volkswirtschaften hinsichtlich ihrer Produktionstechnologien und der Produktivität ihrer Arbeitskräfte sind, desto weniger Anlass besteht für eine Reallokation der Produktionsfaktoren. Unterscheiden sich die Produktionsfunktionen und die Nachfragepräferenzen international nur geringfügig, so dass die Bedingungen des HECKSCHER-OHLIN-SAMUELSON-(HOS)-Modells weitgehend Gültigkeit besitzen, dann ist mit einer substitutiven Beziehung zwischen Güterhandel und internationaler Faktormobilität zu rechnen. Unterscheiden sich die Produktionstechnologien und damit verbunden die Grenzproduktivitäten der Produktionsfaktoren demgegenüber stark, dann bestehen grosse internationale Mobilitätsanreize für die Produktionsfaktoren.

\subsection{FAZIT}

Die theoretische Analyse der Arbeitsmarktperformance der Einwanderer im Rahmen dieses Kapitels hat gezeigt, dass eine Reihe von Faktoren Einfluss auf die Performance der ausländischen Arbeitskräfte besitzen. Sowohl der Selektivität der Zuwanderung, als auch der internationalen Transferierbarkeit von Humankapital kommt grosse Bedeutung zu. Aus den theoretischen Überlegungen ging hervor, dass es sich bei den Zuwanderern i.d.R. um gut ausgebildete Wirtschaftssubjekte handelt, die eine positive Auswahl aus der Gesamtbevölkerung des Herkunftslandes darstellen. Aus diesem Grund ist zu erwarten, dass der migrationsbedingte anfängliche Einkommensrückgang rasch wettgemacht werden kann und die Einwanderer langfristig eine bessere Performance aufweisen werden als die Einheimischen, da sie eine überdurchschnittliche Ausstattung an nicht-beobachtbaren Charakteristika wie Motivation und Ehrgeiz besitzen.

Diese Ergebnisse müssen jedoch insofern relativiert werden, als die Performance der Einwanderer auch stark vom Ausmass der Transferierbarkeit ihres Humanka-

126 Siehe STRAubHAaR (1992). 
pitals ins Zielland abhängt. Je grösser die Transferierbarkeit ist, desto eher ist damit zu rechnen, dass die Einwanderer auf dem Arbeitsmarkt des Ziellandes erfolgreich sein werden. Es ist in diesem Zusammenhang auch davon auszugehen, dass Einwanderer der Zweiten Generation im Zielland i.a. besser integriert sind und aufgrund der im Zielland erworbenen Ausbildung einen höheren Anteil an ziellandspezifischem Humankapital besitzen, so dass mit einer vergleichsweise besseren Performance der Einwanderer der Zweiten Generation zu rechnen ist.

Es ist allerdings darauf hinzuweisen, dass es sich bei der Arbeitsmarktperformance nicht nur um ein statisches, sondern auch um ein dynamisches Phänomen handelt und damit der Zeitdimension eine wichtige Bedeutung zukommt. Es ist daher nicht nur von Interesse, wie sich die Arbeitsmarktperformance zu einem gegebenen Zeitpunkt darstellt, sondern wie sie sich im Zeitverlauf verändert. Es geht also um die Frage der Assimilation der Zuwanderer auf dem Arbeitsmarkt des Ziellandes. Dieser Anpassungsprozess hängt in hohem Masse davon ab, wie ausgeprägt die Investitionen der Einwanderer in ziellandspezifisches Humankapital ausfallen, wobei dies wiederum entscheidend davon beeinflusst wird, ob die $\mathrm{Zu}$ wanderung temporär oder auf Dauer erfolgt.

Letztlich ist darauf hinzuweisen, dass die Arbeitsmarktperformance der Einwanderer auch von einer Reihe intervenierender Faktoren abhängt. Diese Faktoren nehmen sowohl auf die Zusammensetzung der Zuwanderung, als auch, und damit verbunden, auf die Performance der Einwanderer auf dem Arbeitsmarkt des Ziellandes Einfluss. Bei der empirischen Analyse ist daher sowohl dem Einfluss der inländischen Nachfrage nach ausländischen Arbeitskräften, als auch dem Einfluss der rechtlichen Rahmenbedingungen Rechnung zu tragen. Während im Rahmen dieses Kapitels die Bedeutung produktivitätsbedingter Faktoren im Zentrum der Analyse stand, fokussieren sich die Untersuchungen des nachfolgenden Kapitels auf diejenigen Faktoren, die auf diskriminierendes Verhalten insbesondere der Arbeitgeber sowie auf eine Segmentierung des Arbeitsmarktes zurückzuführen sind. 


\section{DISKRIMINIERUNG VON EINWANDERERN AUF DEM ARBEITSMARKT}

Die deskriptive Analyse in Kapitel 3 hat gezeigt, dass zum Teil substantielle Unterschiede in der Arbeitsmarktperformance zwischen Schweizern und Ausländern bestehen. Diese Feststellung trifft sowohl auf die Einkommensverteilung und die Arbeitslosenquoten als auch auf die Beschäftigungsanteile in den verschiedenen sozio-professionellen Kategorien und Wirtschaftszweigen zu. Die Analyse des vorangegangenen Kapitels hat gezeigt, dass den sozio-ökonomischen Charakteristika bzw. der Humankapitalausstattung der einzelnen in- und ausländischen Wirtschaftssubjekte grosser Erklärungswert zukommt. Desweiteren haben die Ausführungen gezeigt, dass auch institutionelle Faktoren wie die rechtlichen Rahmenbedingungen wesentliche Bedeutung besitzen. Schliesslich ist aber nicht auszuschliessen, dass Performanceunterschiede zwischen In- und Ausländern auf eine Diskriminierung der Einwanderer auf dem schweizerischen Arbeitsmarkt zurückzuführen sind.

\section{Abb. 6.1: Arbeitsmarktdiskriminierung induzierende Faktoren}

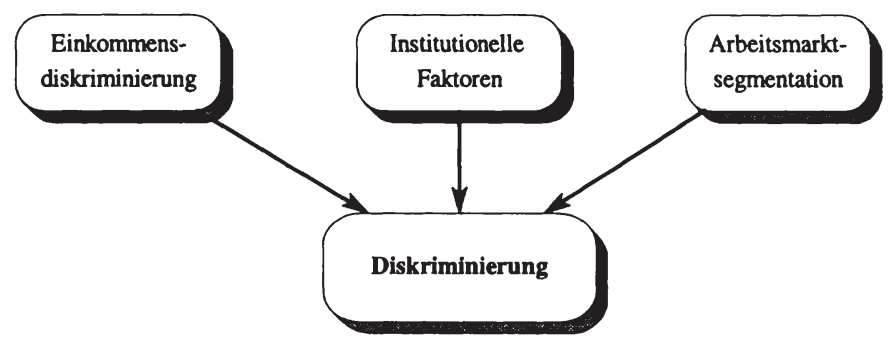

Quelle: Eigene Darstellung

Im Zentrum dieses Kapitels stehen zwei interdependente Ansätze zur Analyse von Diskriminierung: die Einkommensdiskriminierung und die Arbeitsmarktsegmentation (siehe ABB. 6.1). Im Rahmen der Erörterung der Einkommensdiskriminierung wird auf diejenigen ökonomischen Argumente eingegangen, die zur Erklärung von Einkommensdifferentialen herangezogen werden können, und die weder kompensatorischen Charakter besitzen noch produktivitätsbedingt sind. ${ }^{127} \mathrm{Im}$

127 Es handelt sich hierbei um den Ansatz kompensierender Lohndifferentiale der besagt, dass Unterschiede in der sektoralen Lohnstruktur darauf zurückzuführen sind, dass höhere Löhne einen Ausgleich für unvorteilhafte Charakteristika des Arbeitsplatzes darstellen [siehe ROSEN (1986)]. 
Rahmen der Analyse der Arbeitsmarktsegmentation geht es darum, die asymmetrische berufliche und sektorielle Allokation in- und ausländischer Arbeitskräfte sowie den damit verbundenen Einfluss auf die Performance der In- und Ausländer zu erklären. Schliesslich geht auch von institutionellen Faktoren ein Diskriminierungspotential aus.

Das Ziel dieses Kapitels ist es, aus theoretischer Sicht den Stellenwert von Diskriminierung bei der Erklärung der Arbeitsmarktperformance zu erörtern. Die Vorgehensweise gestaltet sich dabei wie folgt. Im ersten Abschnitt wird auf den Begriff der Diskriminierung eingegangen. Im zweiten Abschnitt wird erläutert, welche Ansatzpunkte für Diskriminierung bei der Beschäftigung von Ausländern bestehen. Im dritten Abschnitt wird auf verschiedene theoretische Ansätze zur Erklärung von Einkommensdiskriminierung eingegangen. Im vierten Abschnitt steht die Analyse der Arbeitsmarktsegmentation im Vordergrund und im fünften $A b$ schnitt folgt schliesslich eine Zusammenfassung der potentiellen Wirkungen, die durch Diskriminierung bedingt sein können.

\subsection{BeDEUTUNG UND DEFINITION VON DISKRIMINIERUNG}

Der Begriff 'Diskriminierung' wird im deutschen Sprachgebrauch zur Umschreibung unterschiedlicher Tatbestände verwendet. Diskriminierung kann z.B. das Ergebnis eines Vorurteils sein. Neutraler lässt sich Diskriminierung jedoch als $\boldsymbol{B e}$ nachteiligung interpretieren. Die nachfolgenden Ausführungen stützen sich weitgehend auf die zweite Definition, da diese am ehesten dem in der Arbeitsmarktliteratur vorherrschenden Verständnis von Diskriminierung entspricht. Sehr treffend kommt dies in der Definition von ARROW zum Ausdruck, der unter Diskriminierung "... the valuation in the labor market of personal characteristics of the worker that are unrelated to productivity"128 versteht.

Diese Definition von ARROw berücksichtigt, dass der 'Wert' eines Wirtschaftssubjektes auf dem Arbeitsmarkt sowohl von Angebots- als auch von Nachfragefaktoren abhängt. Aus diesem Grund kann nur dann vom Tatbestand der Diskriminierung ausgegangen werden, wenn nicht-produktivitätsbezogene Faktoren einen positiven oder negativen Effekt besitzen. In der Literatur stellt dabei die Analyse ethnischer bzw. geschlechtsspezifischer Diskriminierung den wichtigsten Untersuchungsgegenstand dar. ${ }^{129}$ Die Analyse von Diskriminierung zwischen Inund Ausländern, d.h. nationalitätenspezifische Diskriminierung, besitzt demge-

128 ARROW (1973a: 3).

129 Empirische Studien zur Lohn- bzw. Einkommensdiskriminierung für die Schweiz finden sich z.B. in AHMAD/FLÜCKIGER (1995), BOYMOND (1993), BRÜDERL/DIEKMANN/ENGELHARDT (1993), CHARLES (1987), DieKMANN/EngelHARDT $(1994,1995)$ und KUGLER (1988). 
genüber sowohl aus theoretischer, als auch aus empirischer Sicht einen geringeren Stellenwert. ${ }^{130}$

Im Zusammenhang mit der Definition von ARROw ist auf drei wichtige Aspekte hinzuweisen, die es im Rahmen dieser Arbeit zu beachten gilt. ${ }^{131}$ Erstens bezieht sich die Identifizierung von Diskriminierung auf messbare Grössen wie das Einkommen, die Löhne, die berufliche Stellung oder die Beschäftigungssituation. Vorurteile gegenüber einer bestimmten Gruppe können von den Mitgliedern dieser Gruppe zwar wahrgenommen werden, diese Gefühle müssen jedoch von Handlungen begleitet sein, die das Marktergebnis beeinflussen, damit im Rahmen dieser Studie von Diskriminierung gesprochen wird. Zweitens stehen im Zentrum der Analyse dieses Kapitels lediglich jene Unterschiede, welche systematischer und nicht zufälliger Natur sind, sich also nicht innerhalb einer grossen Gruppe ausgleichen. Drittens gilt es zu beachten, dass Unterschiede in der Arbeitsmarktperformance zwischen zwei Gruppen, d.h. in unserem Fall zwischen In- und Ausländern, sowohl durch sog. 'vor-arbeitsmarktliche' als auch durch 'arbeitsmarktliche' Faktoren bedingt sein können. ${ }^{132}$ Diskriminierung kann daher sowohl bei der Humankapitalakkumulation als auch bei der Humankapitalentgeltung stattfinden, wobei im Rahmen der vorliegenden Studie der zweite Aspekt im Vordergrund steht. Es gilt allerdings den Einfluss vor-arbeitsmarktlicher Faktoren mit zu berücksichtigen, da ansonsten eine Überschätzung der auf arbeitsmarktlichen Faktoren basierenden Diskriminierung stattfinden kann. Diese Feststellung ist insbesondere aus wirtschaftspolitischer Sicht von grosser Bedeutung, da ein effizientes Vorgehen gegen Diskriminierung nur dann möglich ist, wenn Klarheit über die genauen Ursachen der Diskriminierung besteht.

130 Viele Studien, vor allem aus den Vereinigten Staaten, fokussieren in ihrer Analyse auf die Messung des Einflusses der Zugehörigkeit zu einer ethnischen Minoritätengruppe wie der spanischstämmigen oder der asiatischen Bevölkerung oder auf Unterschiede zwischen weissen und schwarzen Arbeitnehmern. Eine Vielzahl an Studien beschäftigt sich daneben mit der Analyse geschlechtsspezifischer Diskriminierung [siehe dazu die Übersichtsartikel von BLAU/FERBER (1987), CAIN (1986) und GLl (1994)].

131 Siehe auch EHRENBERG/SMITH (1994).

132 KAUFMAN (1989: 397) definiert diese beiden Faktoren wie folgt: "Premarket discrimination denies to those who are discriminated against an equal opportunity to develop their abilities and talents during their formative, pre-employment years. ... Market discrimination takes place when people of equal capabilities who are competing in the labor market are given unequal job assignments, promotions or rates of pay solely on the basis of some characteristic that is unrelated to their performance." 


\subsection{ANSATZPUNKTE FÜR DISKRIMINIERUNG BEI DER BESCHÄFTI- GUNG VON EINWANDERERN}

Die Ausführungen des vorangegangen Abschnitts haben verdeutlicht, dass einerseits eine klare Trennung zwischen Produktivitäts- und Diskriminierungsfaktoren vorgenommen werden muss, dass sich Diskriminierung aber andererseits auf eine Reihe unterschiedlicher Arbeitsmarktgegebenheiten auswirken kann. Die wichtigsten Diskriminierungstatbestände sind in TAB. 6.1 zusammengefasst. Im Sinne einer Abgrenzung des vorangegangenen Abschnitts lassen sich arbeitgeberseitige und institutionell bedingte Diskriminierung unterscheiden, die jeweils von unterschiedlicher Relevanz für die einzelnen Diskriminierungstatbestände sind. Diese sind dabei nicht als voneinander unabhängige Folgen von Diskriminierung zu interpretieren, sondern müssen als interdependentes System verstanden werden.

Die nachfolgenden Ausführungen konzentrieren sich auf die Analyse von Diskriminierung hinsichtlich der Einkommenshöhe und der Berufswahl, d.h. Einkommensdiskriminierung und Arbeitsmarktsegmentation bzw. branchenspezifische Segregation. Diskriminierung hinsichtlich der Einstellungs- bzw. Beschäftigungschancen sowie der Arbeitsbedingungen und Aufstiegschancen gegenüber (angeblich) benachteiligten Gruppen werden vor allem im Rahmen der Ausführungen zur Arbeitsmarktsegmentation behandelt. ${ }^{133}$ Im ersten Fall ist davon auszugehen, dass während konjunktureller Einbrüche zuerst Ausländer entlassen werden und während Phasen des Aufschwungs und der damit verbundenen Zunahme der Arbeitsnachfrage zuerst Inländer eingestellt werden. Im zweiten Fall kann sich Diskriminierung einerseits in schlechteren Arbeitsbedingungen der ausländischen Arbeitskräfte äussern sowie andererseits zu einer Konzentration der Ausländerbeschäfti-

133 Zur Analyse dieser Tatbestände wird i.d.R. auf spezielle Experimente zurückgegriffen [siehe VELLING (1995b) sowie YINGER (1986), auf den diese Untersuchungsmethode zurückgeht]. In einem ersten Schritt werden dabei Paare von Versuchspersonen ausgewählt, die sich in allen wichtigen Produktivitätsmerkmalen gleichen. Sie unterscheiden sich lediglich dadurch, dass der eine der nicht-benachteiligten und der andere der (angeblich) benachteiligten Gruppe angehört. Diese Paare werden dann über einen längeren Zeitraum hinsichtlich ihrer Arbeitsmarkterfahrung beobachtet, um so allfällige Benachteiligungen im Zeitverlauf eindeutig erkennen zu können. Unterschiede innerhalb eines Paares deuten dann auf Diskriminierung hin.

Der Vorteil dieser Art der Untersuchung besteht in der Kontrolle über das Experimentdesign. Ebenfalls ist die Vergleichbarkeit der jeweils ein Paar bildenden Versuchspersonen weitgehend gewährleistet. Desweiteren lässt sich der Einfluss institutioneller Faktoren relativ gut eingrenzen. Das zentrale Problem besteht allerdings darin, dass aufgrund des relativ hohen Aufwandes die Untersuchung auf eine geringe Fallzahl beschränkt bleibt, womit sich die Frage nach der Repräsentativität stellt und der gesamtwirtschaftlichen Relevanz der Ergebnisse. Aus diesen Gründen ist diese Art der Untersuchung bislang eher selten Anwendung durchgeführt worden. 
gung in unteren Hierarchieebenen bzw. einer branchenspezifischen Segregation führen. ${ }^{134}$

Tab. 6.1: Ansatzpunkte zur Diskriminierung ausländischer Arbeitnehmer

\begin{tabular}{|c|c|c|c|c|}
\hline & \multicolumn{4}{|c|}{ (Mögliche) Diskriminierungstatbestände } \\
\hline & $\begin{array}{c}\text { Einstellungs- bzw. } \\
\text { Beschäftigung- } \\
\text { schancen }\end{array}$ & Berufswahl & $\begin{array}{c}\text { Arbeitsbedingun- } \\
\text { gen / Aufstiegs- } \\
\text { chancen }\end{array}$ & Einkommenshöhe \\
\hline $\begin{array}{l}\text { Arbeitge- } \\
\text { berseitig }\end{array}$ & $\begin{array}{l}\text { - seltenere tat- } \\
\text { sächliche Ein- } \\
\text { stellung } \\
\text { - höhere Entlas- } \\
\text { sungswahr- } \\
\text { scheinlichkeit }\end{array}$ & $\begin{array}{l}\text { - branchen- } \\
\text { /tätigkeitsspezifi- } \\
\text { sche Rekrutie- } \\
\text { rung ausländi- } \\
\text { scher Arbeits- } \\
\text { kräfte }\end{array}$ & $\begin{array}{l}\text { - häufiges Einset- } \\
\text { zen bei unange- } \\
\text { nehmer Arbeit } \\
\text { (Fliessband, } \\
\text { Schicht- und } \\
\text { Nachtarbeit) } \\
\text { - weniger häufige } \\
\text { Beförderungen }\end{array}$ & $\begin{array}{l}\text { - Einstufung in tie- } \\
\text { fere Einkom- } \\
\text { mensklassen bei } \\
\text { der Einstellung } \\
\text { - Vorenthaltung } \\
\text { von aussertarifli- } \\
\text { chen Leistungen }\end{array}$ \\
\hline $\begin{array}{l}\text { Institutio- } \\
\text { nell bedingt }\end{array}$ & $\begin{array}{l}\text { - Inländervorrang } \\
\text { - Einwanderungs- } \\
\text { kontingentierung } \\
\text { - Rekrutierungs- } \\
\text { prioritäten }\end{array}$ & $\begin{array}{l}\text { - Beschränkter } \\
\text { Zugang zur selb- } \\
\text { ständigen Er- } \\
\text { werbstätigkeit } \\
\text { sowie Beschäfti- } \\
\text { gung im öffentli- } \\
\text { chen Sektor } \\
\text { - Einschränkungen } \\
\text { im Stellen-/ Be- } \\
\text { rufs- und Kan- } \\
\text { tonswechsel } \\
\end{array}$ & $\begin{array}{l}\text { - Inländervorrang } \\
\text { - Einwanderungs- } \\
\text { kontingentierung } \\
\text { - Einschränkungen } \\
\text { im Stellen-/ Be- } \\
\text { rufs- und Kan- } \\
\text { tonswechsel }\end{array}$ & $\begin{array}{l}\text { - Verboten gemäss } \\
\text { Art. 9, Abs. } 1 \\
\text { BVO: Prinzip } \\
\text { gleicher Lohn- } \\
\text { und Arbeitsbe- } \\
\text { dingungen }\end{array}$ \\
\hline $\begin{array}{l}\text { Mögliche } \\
\text { Folge }\end{array}$ & $\begin{array}{l}\text { - höhere Arbeits- } \\
\text { losigkeit } \\
\text { - geringere Er- } \\
\text { werbsbeteiligung }\end{array}$ & $\begin{array}{l}\text { - Arbeitsmarkt- } \\
\text { segmentation }\end{array}$ & $\begin{array}{l}\text { - schlechtere Ar- } \\
\text { beitsbedingun- } \\
\text { gen } \\
\text { - Konzentration in } \\
\text { unteren Hierar- } \\
\text { chieebenen }\end{array}$ & $\begin{array}{l}\text { - Einkommens- } \\
\text { diskriminierung }\end{array}$ \\
\hline
\end{tabular}

Quelle: Eigene Darstellung nach VELLING (1995a)

Aus TAB. 6.1 geht hervor, dass aus theoretischer Sicht eine grundsätzliche Unterscheidung zwischen arbeitgeberseitiger und institutionell bedingter Diskriminierung vorgenommen werden kann, die jeweils unterschiedliche Diskriminierungstatbestände reflektiert. ${ }^{135}$ Die Ausführungen dieses Kapitels befassen sich in erster

134 Besitzen schweizerische Arbeitnehmer z.B. bei gleicher Qualifikation bessere Aufstiegschancen als ausländische Arbeitnehmer, so ist davon auszugehen, dass das Lebenseinkommen der Schweizer über jenem der Ausländer liegen wird, auch wenn zum gegenwärtigen Zeitpunkt keine Lohn- oder Einkommensunterschiede bestehen mögen.

135 CAIN (1986: 729) verweist in seinem Übersichtsartikel darauf, dass insbesondere MARSHALL (1974) dafür plädierte, institutionelle Theorien der Diskriminierung als alternative Erklärungsansätze zu den neoklassischen Theorien der Lohndiskriminierung anzusehen. 
Linie mit der Analyse arbeitgeberseitiger Diskriminierung. Den institutionellen Faktoren kommt bei der Erklärung der Diskriminierung der Einwanderer auf dem Arbeitsmarkt aber ebenfalls wesentliche Bedeutung zu. ${ }^{136}$

Erstens findet aufgrund der Kontingentierung, der Rekrutierungsprioritäten sowie des Inländervorrangs bereits vor der Einwanderung eine Diskriminierung gegenüber gewissen potentiellen Einwanderungsgruppen statt, denen eine Einreise in die Schweiz zum Zweck der Erwerbstätigkeit nicht gestattet wird. ${ }^{137}$ Zweitens werden auch in der Schweiz lebende Ausländer institutionell diskriminiert, indem ihnen der Zugang zu gewissen Berufen, insbesondere im öffentlichen Sektor, verwehrt bleibt, indem die Möglichkeit einer selbständigen Erwerbstätigkeit stark eingeschränkt und der Berufs-, Kantons- und Stellenwechsel, je nach Aufenthaltskategorie, erschwert ist. ${ }^{138}$ Drittens ist davon auszugehen, dass die Inländer grösseren Einfluss auf die Gestaltung und Veränderung des institutionellen Rahmenwerks bzw. der gesetzlichen Bestimmungen des Ausländerrechts nehmen, da sie die Bevölkerungsmehrheit ausmachen und die Ausländer i.d.R. keine oder nur sehr eingeschränkte politische Rechte besitzen. Die Anliegen der ausländischen Bevölkerung werden daher nicht direkt in der Zielfunktion stimmenmaximierender Politiker berücksichtigt. ${ }^{139}$

Abschliessend ist darauf hinzuweisen, dass institutionelle Faktoren zwar die Beschäftigungsstruktur mitbestimmen, den Lohnsetzungsprozess aber nicht direkt beeinflussen, da die rechtlichen Bestimmungen nur einen geringen Spielraum für eine unterschiedliche Entlohnung von In- und Ausländern zulassen. ${ }^{140}$ Indirekt nehmen institutionelle Faktoren jedoch, über die Eingrenzung des 'set of opportunity', erheblichen Einfluss auf die Beschäftigungs- und Einkommensperformance der In- und Ausländer. Auf eine Unterscheidung institutioneller und verhaltensbedingter Diskriminierung wird allerdings im Rahmen der empirische Analyse verzichtet, da eine klare Trennung dieser beiden Aspekte i.d.R. nicht möglich ist.

136 Auf den Einfluss institutioneller Faktoren wurde bereits in Kapitel 2 sowie in Abschnitt 5.4.2 ausführlich eingegangen.

137 Dieser Aspekt der Rassendiskriminierung der schweizerischen Migrationspolitik ist insbesondere von der Antirassismuskommission aufgegriffen worden und als Argument gegen das Drei-Kreise-Modell angeführt worden [siehe WEHRLI (1996)].

138 Siehe Kapitel 2.

139 Siehe auch Abschnitt 6.3.3.3.

140 Gemäss Art. 9 Abs. 1 BVO gilt das Prinzip gleicher Lohn- und Arbeitsbedingungen für Inund Ausländer. 


\subsection{EINKOMMENSDISKRIMINIERUNG}

Aus theoretischer Sicht werden i.a. drei verschiedene Ursachen für das Entstehen von Einkommensdiskriminierung angeführt. ${ }^{141}$ Erstens können Vorurteile der Arbeitgeber, anderer Arbeitnehmer oder der Kunden zu einer Einkommensdiskriminierung gegenüber ausländischen Erwerbstätigen führen. Zweitens kann Einkommensdiskriminierung auf die Existenz unvollständiger Information zurückgeführt werden. In diesem Zusammenhang wird von sog. statistischer Diskriminierung gesprochen. Drittens lässt sich Diskriminierung über die asymmetrische Machtverteilung zwischen Arbeitgebern und Arbeitnehmern erklären.

Bei den hier betrachteten Ansätzen handelt es sich um nachfrageseitige Theorien. Die Angebotsseite wird weitgehend vernachlässigt, weil unterstellt wird, dass sich die diskriminierten bzw. nicht-diskriminierten Arbeitskräfte in ihrer Produktivität nicht unterscheiden und dass sie zudem identische Arbeitsangebotspräferenzen aufweisen. Die Nachfrageseite lässt sich demgegenüber durch eine wettbewerbliche oder monopolistische und eine exakte oder stochastische Struktur charakterisieren. ${ }^{142}$

\subsubsection{Persönliche VorURTEILE}

Die erste Quelle für Diskriminierung stellen persönliche Vorurteile dar. Die Auswirkungen von Vorurteilen auf Einkommens- und Beschäftigungsmuster wurden erstmals von BECKER (1971) thematisiert. Als Ausgangspunkt diente BECKER die Überlegung, dass Vorurteile einer Majoritätengruppe, in unserem Falle der Inländer, gegenüber einer Minoritätengruppen, in unserem Falle den Ausländern, zu Nutzeneinbussen führen, die über Preis- bzw. Lohnabschläge abgegolten werden müssen. BECKER ging in diesem Zusammenhang davon aus, dass es sich bei diesen Betroffenen um drei verschiedene Gruppen handelt: Arbeitgeber, Arbeitnehmer und Kunden.

\subsubsection{Vorurteile von Arbeitgebern}

Besitzt ein Arbeitgeber, der Terminologie von BECKER folgend einen sog. 'taste for discrimination' gegenüber Ausländern, dann sieht er sich bei der Beschäftigung eines Ausländers effektiven Kosten in Höhe von $w_{f}\left(1+d_{i}\right)$ gegenüber, d.h. dem Lohn der ausländischen Arbeitskraft $w_{f}$ zuzüglich eines Diskriminierungskoeffizienten $d_{i}$. Dieser Koeffizient spiegelt das Ausmass des negativen Nutzens wider, das sich für den inländischen Arbeitgeber aus der Beschäftigung eines ausländischen Arbeitnehmers ergibt. Die Implikationen, die sich aufgrund arbeitge-

141 Siehe z.B. EHRENBERG/SMITH (1994). Diese Ansätze wurden i.d.R. zur Analyse und Erklärung ethnischer und geschlechtsspezifischer Diskriminierung entwickelt.

142 Siehe CAIN (1986). 
berseitiger Vorurteile hinsichtlich der Einkommen und Beschäftigung ergeben, lassen sich am besten anhand einer Graphik veranschaulichen.

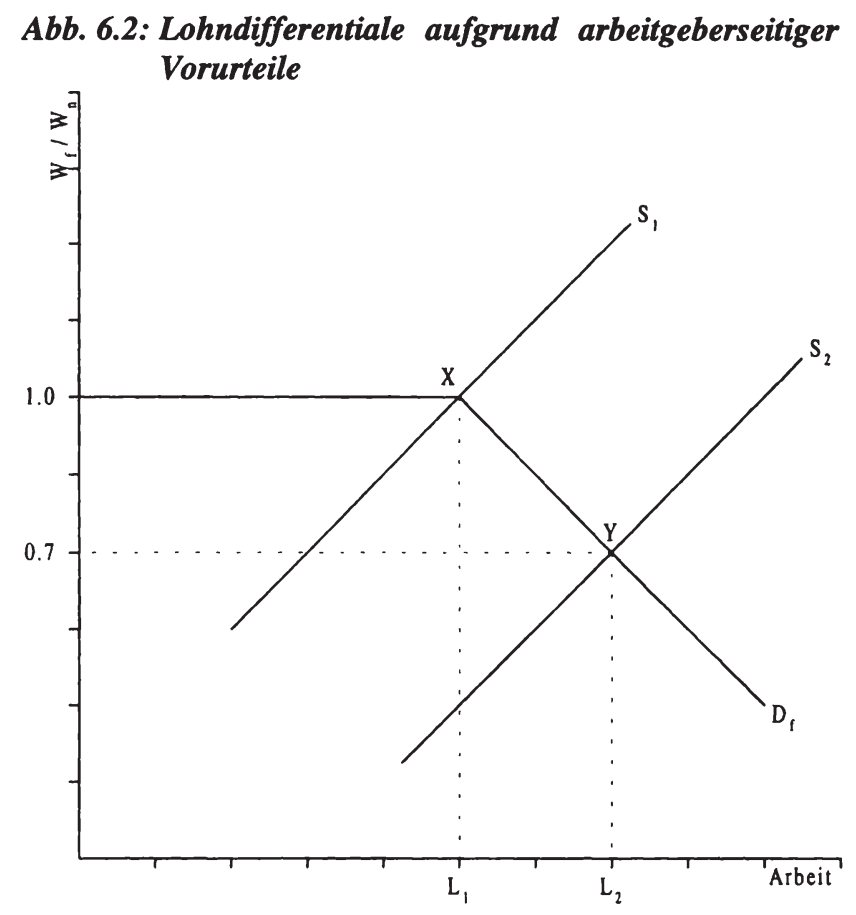

Quelle: Eigene Darstellung nach KaUfMaN (1989)

In ABB. 6.2 misst die Y-Achse das Lohnverhältnis zwischen Aus- und Inländern und die X-Achse die Beschäftigung ausländischer Arbeitskräfte. Im Punkt $\mathrm{w}_{\mathrm{f}} / \mathrm{w}_{\mathrm{n}}=1$ befinden sich die Löhne von In- und Ausländern auf demselben Niveau. Die Nachfragekurve $D_{f}$ bildet die Gesamtnachfrage nach ausländischen Arbeitskräften bei unterschiedlichen Löhnen ab. Die Form der Nachfragekurve ergibt sich dabei aus der Aneinanderreihung der individuellen Arbeitsnachfragen der verschiedenen inländischen Arbeitgeber unter Berücksichtigung der jeweiligen Diskriminierungskoeffizienten. Es werden dabei zuerst die Arbeitsnachfragen derjenigen Arbeitgeber abgebildet, die den kleinsten Koeffizienten $d_{i}$ besitzen. Der horizontale Abschnitt von $D_{f}$ entspricht daher der Arbeitsnachfrage der nicht-diskriminierenden Arbeitgeber $\left(\mathrm{d}_{\mathrm{i}}=0\right)$. Da im Rahmen dieses Kapitels unterstellt wird, dass In- und Ausländer gleich produktiv sind, werden nicht-diskriminierende Arbeitgeber bereit sein, Ausländer einzustellen, solange $w_{f} \leq w_{n}$. Auf dem negativ geneigten Ast der Nachfragekurve führen zunehmende Vorurteile der Arbeitgeber 
dazu, dass zusätzliche ausländische Arbeitskräfte nur dann eingestellt werden, wenn diese einen fortwährend geringeren relativen Lohn akzeptieren.

Das Modell von BECKER impliziert, dass das Ausmass des Lohndifferentials zwischen In- und Ausländern von zwei Faktoren bestimmt wird. Erstens ist die Grösse der ausländischen Bevölkerung von Bedeutung. Aus ABB. 6.2 geht hervor, dass der relative Lohn der ausländischen Arbeitskräfte umso tiefer ausfällt, je grösser das ausländische Arbeitsangebot bei gegebener Nachfrage ist. Im Falle der Angebotskurve $S_{1}$ werden alle Ausländer eine Beschäftigung bei nicht-diskriminierenden Arbeitgebern finden. Weitet sich das Angebot aus und verschiebt sich somit die Angebotskurve nach $S_{2}$, dann muss der relative Lohn auf 0.7 fallen, um inländische Arbeitgeber dazu zu bewegen, ausländische Arbeitskräfte zu beschäftigen. Zweitens hängt das Ausmass des Lohndifferentials auch vom Anteil der vorurteilsbehafteten Arbeitgeber ab. In einer Situation ohne arbeitgeberseitige Vorurteile wäre die Arbeitsnachfragekurve vollständig elastisch, und es würde keine Lohndiskriminierung stattfinden. Je grösser demgegenüber die Zahl der diskriminierenden Arbeitgeber ist, desto weiter nach links verschiebt sich die Arbeitsnachfragekurve und umso geringer fällt der damit verbundene relative Lohn der ausländischen Arbeitskräfte aus. ${ }^{143}$

Aus dem Modell von BECKER lassen sich zwei interessante Aussagen ableiten. ${ }^{144}$ Erstens führen die Vorurteile der Arbeitgeber zu einer vollständigen Segregation der in- und ausländischen Arbeitskräfte zwischen den verschiedenen Unternehmen. Wird von der Arbeitsangebotskurve $S_{2}$ der Minoritätengruppe der Ausländer in ABB. 6.2 ausgegangen, so werden Arbeitgeber links von Punkt Y nur ausländische und Arbeitgeber rechts von $\mathrm{Y}$ nur inländische Arbeitskräfte beschäftigen. Links von Punkt $Y$ überkompensiert der tiefe relative Lohn der Ausländer die Vorurteile der Arbeitgeber und da unterstellt wird, dass In- und Ausländer gleich produktiv sind, werden nur ausländische Arbeitnehmer beschäftigt. Rechts von $\mathrm{Y}$ werden dementsprechend nur inländische Arbeitskräfte eingestellt, da die Vorurteile der Arbeitgeber durch die relativ tieferen Löhne der ausländischen Arbeitskräfte nicht kompensiert werden können.

Zweitens ergibt sich aus dem Modell von BECKER die Schlussfolgerung, dass die diskriminierungsbedingten Lohndifferentiale zwischen In- und Ausländern im Zeitverlauf verschwinden werden, weil vorurteilsbehaftete Arbeitgeber aus dem Markt gedrängt werden. Dieses Ergebnis hängt damit zusammen, dass Vorurteile für diskriminierende Arbeitgeber mit Opportunitätskosten verbunden sind, da sie

143 Es ist dabei denkbar, dass das Ausmass der Vorurteile gegenüber ausländischen Arbeitskräften positiv mit der Grösse der ausländischen Bevölkerung korreliert ist. Dies würde auf eine zusätzliche Reduktion der Löhne der ausländischen Arbeitnehmer hindeuten, da sich die Angebotskurve in ABB. 6.2 weiter nach rechts und die Nachfragekurve weiter nach links verschieben würde.

Siehe CAIN (1986), KAUFMAN (1989) sowie EHRENBERG/SMITH (1994). 
gleich produktive Ausländer zu einem tieferen Lohn beschäftigen könnten. In einer Marktwirtschaft hat dieser reale Kostenvorteil der nicht-diskriminierenden Arbeitgeber eine Verdrängung der diskriminierenden Arbeitgeber zur Folge. Diese beiden Ergebnisse decken sich aber in dieser Form nicht mit der Realität. ${ }^{145}$ Erstens besteht die Möglichkeit, dass Firmen in oligopolistischen oder regulierten Märkten tätig sind, so dass ein gewisser Spielraum für Diskriminierung besteht. Zweitens kann vor-arbeitsmarktliche Diskriminierung dazu führen, dass kein ausreichendes Angebot an gut qualifizierten ausländischen Arbeitskräften besteht. ${ }^{146}$ Drittens ist schliesslich zu vermuten, dass sich in Zeiten erhöhter Arbeitslosigkeit die Diskriminierungskosten verringern, weil die inländischen Arbeitskräfte bereit sind, zu tieferen Löhnen zu arbeiten.

\subsubsection{Vorurteile der Arbeitnehmer}

Gehen die Vorurteile von den Arbeitnehmern der Majoritätengruppe aus, so werden diese eine Zusammenarbeit mit den Arbeitnehmern der Minoritätengruppen zu vermeiden versuchen. Im Modell von BECKER äussert sich arbeitnehmerseitige Diskriminierung darin, dass sich $w_{n}$, der Lohn der Inländer, durch die Zusammenarbeit mit Ausländern um $d_{k}$, die arbeitnehmerspezifische Diskriminierungskomponente reduziert und damit der Nettolohn lediglich $w_{n}\left(1-d_{k}\right)$ entspricht. Die inländischen Arbeitnehmer werden somit nur dann bereit sein, mit Ausländern zusammenzuarbeiten, wenn sie einen Lohnaufschlag in Höhe von $w_{n} d_{k}$ erhalten. Die Arbeitgeber erwarten daher zusätzliche Kosten, wenn sie sowohl in- als auch ausländische Arbeitskräfte beschäftigen wollen. Langfristig ergeben sich in diesem Modell somit dieselben Implikationen wie im Modell arbeitgeberseitiger Diskriminierung, die sich jedoch, wie bereits erwähnt, nicht mit der Realität decken. Erstens ist von einer vollständigen Segregation der in- und ausländischen Arbeitskräfte zwischen den einzelnen Firmen auszugehen, und zweitens ist im Zeitverlauf mit einem Verschwinden der diskriminierenden Lohndifferentiale zu rechnen.

\subsubsection{Vorurteile der Kunden}

Gehen die Vorurteile schliesslich von den Kunden aus, so werden diese ein Produkt oder eine Dienstleistung eines Unternehmens, das Ausländer beschäftigt, dann nachfragen, wenn sie über tiefere Preise dafür kompensiert werden, dass das Produkt bzw. die Dienstleistung von einem Ausländer hergestellt bzw. erbracht wurde. Um diesem Umstand Rechnung zu tragen, müssen die ausländischen Ar-

145 Siehe KaUfman (1989).

146 In einer empirischen Studie über den Bankensektor kamen ASHENFELTER/HANNAN (1986) zum Ergebnis, dass sich der Anteil der weiblichen Beschäftigten in den Unternehmen reduzierte, je geringer die Zahl der miteinander im Wettbewerb stehenden Banken innerhalb eines geographischen Gebietes war. 
beitskräfte entweder tiefere Löhne akzeptieren oder im Mittel besser qualifiziert sein als Inländer, damit sie eine Beschäftigung finden. Diese Situation ist jedoch instabil, da ausländische Arbeitskräfte in Tätigkeiten ohne Kundenkontakt ein höheres Einkommen erzielen könnten. Langfristig würden somit zwar die Lohndifferentiale beseitigt, gleichzeitig würde aber auch eine partielle berufliche Segregation zwischen in- und ausländischen Arbeitnehmern stattfinden.

Die bisherigen Ausführungen, die sich weitgehend auf BECKER (1971) abstützten, beziehen sich ausschliesslich auf Lohn- bzw. Einkommensdiskriminierung im Rahmen eines neoklassischen Modells mit markträumenden Lohnsätzen. Wird, davon abweichend, unterstellt, dass im Gleichgewicht keine Markträumung besteht und somit (unfreiwillige) Arbeitslosigkeit existiert, kann sich Diskriminierung auch implizit darin äussern, dass inländische Arbeitnehmer bei der Neueinstellung bevorzugt werden und daher schneller eine Beschäftigung finden, und/oder dass sie im Falle rezessiver Tendenzen bessere (Weiter-)Beschäftigungschancen und damit ein geringeres Arbeitslosigkeitsrisiko besitzen als Ausländer. ${ }^{147}$ Dies wird umso eher der Fall sein, je stärker der Spielraum für Lohn- bzw. Einkommensdifferenzierung, z.B. aus tarifvertraglichen Gründen oder aufgrund der Rechtslage, eingeschränkt ist. In diesem Fall können Vorurteile gegenüber Ausländern nicht mehr durch Lohn- oder Einkommensabschläge kompensiert werden, sondern können sich nur in eine unterschiedliche Einstellungs- bzw. Beschäftigungspraxis äussern. ${ }^{148}$

\subsubsection{UNVOLLSTÄNDIGE INFORMATION}

Ein zweiter Ansatz zur Erklärung von Diskriminierung auf dem Arbeitsmarkt stellt die sog. statistische Diskriminierung dar. ${ }^{149}$ Diskriminierung ist in diesem Zusammenhang als Ergebnis unvollständiger Information während des sog. 'screening'-Prozesses zu verstehen. Als Ausgangspunkt dient die Überlegung, dass Arbeitgeber bei der Einstellung neuer Mitarbeiter i.a. keine vollständigen Informationen über deren effektive Produktivität besitzen. Die Arbeitgeber sind daher gezwungen, aus den verfügbaren Informationen über die persönlichen Charakteristika der einzelnen Bewerber Hinweise auf deren Produktivität zu gewinnen. Einige dieser Charakteristika sind individueller Natur wie das Ausbildungsniveau oder die Arbeitserfahrung, andere jedoch gruppenspezifischer, wie das Geschlecht oder die Nationalität. Die Berücksichtigung solcher Merkmale im Rahmen eines 'screening'-Prozesses kann zu statistischer Diskriminierung führen, wenn Wirt-

147 Eine höhere Arbeitslosigkeitswahrscheinlichkeit der Ausländer kann aber auch das Ergebnis einer ungünstigeren sektoriellen Verteilung der Ausländer im Vergleich zu den Inländern sein [siehe Abschnitt 6.4 sowie PIORE (1979)].

Siehe VELLING (1995a).

149 siehe AIGNER/CAIN (1977). Eine ähnliche Theorie wurde von SPENCE (1973) entwickelt. 
schaftssubjekte mit denselben messbaren individuellen Charakteristika aufgrund der Zugehörigkeit zu verschiedenen Gruppen systematisch unterschiedlich bewertet werden. ${ }^{150}$

\section{$A b b$. 6.3: Häufigkeitsverteilung der Arbeitsproduktivität von In- und Ausländern}

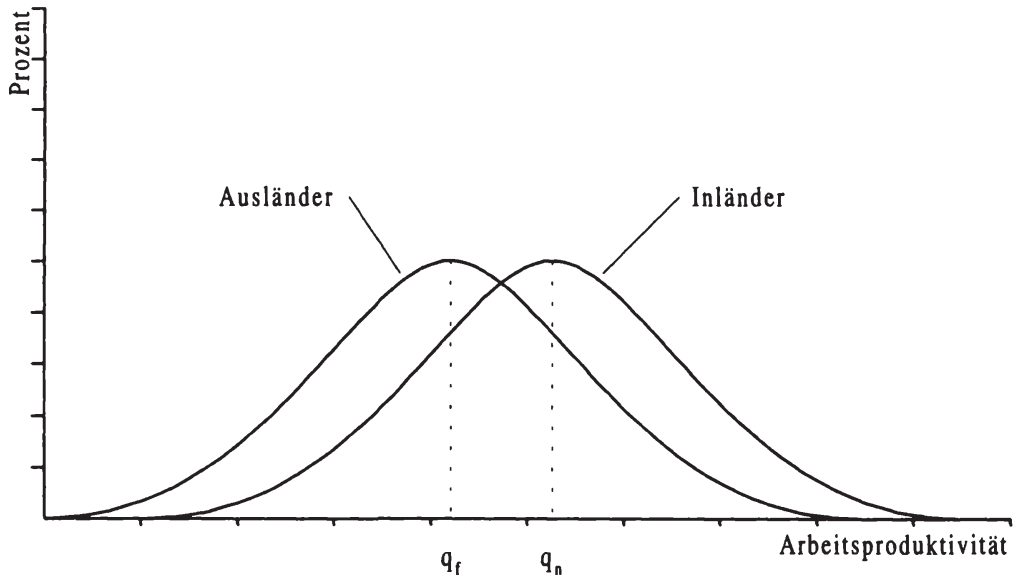

Quelle: Eigene Darstellung nach EHRENBERG/SMITH (1994)

Wird wie in ABB. 6.3 unterstellt, dass ausländische im Vergleich zu inländischen Arbeitskräften eine tiefere durchschnittliche Arbeitsproduktivität aufweisen $\left(q_{r}<q_{n}\right)^{151}$, und wird zudem davon ausgegangen, dass den Arbeitgebern zwar Informationen über die gruppenspezifischen, nicht aber die individuellen Merkmale zur Abschätzung der Arbeitsproduktivität zur Verfügung stehen, dann werden inländische gegenüber gleich produktiven ausländischen Arbeitskräften systematisch bevorzugt. Auf zwei Aspekte ist in diesem Zusammenhang speziell hinzuweisen. Erstens ist Diskriminierung in diesem Fall nicht das Ergebnis von Vorurteilen, sondern ergibt sich aufgrund unvollständiger Informationen der Arbeitgeber. Zweitens sind Einkommensunterschiede auf Gruppenebene nicht das Resultat von Diskriminierung, sondern spiegeln die tatsächlichen Produktivitätsunterschiede zwischen verschiedenen Gruppen wider. Auf individueller Ebene lassen sich Einkommensunterschiede demgegenüber auf Diskriminierung zurückführen.

150 Siehe OETTINGER (1996). EHRENBERG/SMITH (1994: 421) bemerken hierzu: "Statistical discrimination can be viewed as a part of the screening problem, which arises when observable personal characteristics that are correlated with productivity are not perfect predictors".

Diese Annahme lässt sich z.B. auf vor-arbeitsmarktliche Diskriminierung oder die unvollständige Transferierbarkeit des Humankapitals der Einwanderer zurückführen. Sie entspricht auch den Ergebnissen der deskriptiven Analyse in Kapitel 3. 
Wie im Falle von Informationsasymmetrien ist zu erwarten, dass sich statistische Diskriminierung im Zeitverlauf verringert.

Statistische Diskriminierung ist jedoch nicht auf Informationsasymmetrien hinsichtlich der Arbeitsproduktivität beschränkt, sondern kann sich auch auf weitere Aspekte erstrecken, die das Verhältnis zwischen der Produktivität der Arbeitnehmer und den für das Unternehmen aus dem Beschäftigungsverhältnis erwachsenden Kosten tangieren. ${ }^{152}$ Dieser Zusammenhang ist insbesondere im Rahmen von Untersuchungen zur geschlechtsspezifischen Diskriminierung von Frauen von Bedeutung, wenn Arbeitgeber damit rechnen müssen, dass Frauen im gebärfähigen Alter nach kurzer Zeit wieder aus dem Unternehmen ausscheiden. ${ }^{153}$ Die Kosten der Einarbeitung und Einstellung von Frauen müssen in diesem Fall während eines kürzeren Zeitraums amortisiert werden und erhöhen dadurch die Kosten der Beschäftigung von Frauen. Analog dazu muss bei der Beschäftigung von Ausländern damit gerechnet werden, dass diese im Falle temporärer Zuwanderung nach kurzer Zeit bereits wieder in ihre Herkunftsländer zurückkehren, was zur gleichen Schlussfolgerung führt.

Es ist schliesslich darauf hinzuweisen, dass die Verwendung gruppenspezifischer Informationen zur Abschätzung der effektiven individuellen Arbeitsproduktivitäten umso höhere Opportunitätskosten verursacht, je stärker sich die einzelnen Gruppenangehörigen voneinander unterscheiden. Nimmt z.B. der Anteil der Frauen $a b$, die aus familiären Gründen für eine gewisse Zeit aus dem Arbeitsmarkt ausscheiden und verwenden die Arbeitgeber weiterhin das Geschlecht der Arbeitnehmer als Massstab für die Arbeitsmarktbindung, so werden viele Frauen, die erwerbstätig bleiben möchten, nicht beschäftigt, während gleichzeitig männliche Bewerber eingestellt werden, die weniger produktiv sind. Zusammenfassend lässt sich festhalten, dass "... as premarket differences between the races or sexes narrow, the use of race or sex group data should lessen and statistical discrimination should gradually disappear."154

\subsubsection{MARKTMACHT}

Ein dritter Ansatz zur Erklärung von Diskriminierung ergibt sich aus der Ausübung von Marktmacht der verschiedenen Akteure auf dem Arbeitsmarkt. Diese Marktmacht kann erstens von monopsonistischen Unternehmen auf der Nachfrageseite ausgehen, zweitens von Gewerkschaften auf der Angebotsseite oder drittens von der Regierung, die aufgrund ihrer hoheitlichen Rechte ebenfalls erheblichen Einfluss auf die Arbeitsmarktperformance der In- und Ausländer ausüben

152 Siehe VELLING (1995a).

153 Siehe THUROW (1975).

154 EHRENBERG/SMITH (1994: 423). 
kann. ${ }^{155}$ Das Motiv für Diskriminierung, zumindest was Unternehmen und Gewerkschaften betrifft, liegt in diesem Fall weder in Vorurteilen noch in unvollständiger Information begründet, sondern in der Erzielung zusätzlichen Gewinns mittels Diskriminierung.

Im Gegensatz zu den bisher diskutierten Ansätzen, die zwar eine Erklärung für das Entstehen nicht jedoch für die längerfristige Persistenz von Diskriminierung zu geben vermochten, lässt sich dieser Umstand im Rahmen des auf Marktmacht beruhenden Ansatzes plausibel erklären, da sich Angehörige der Majoritätengruppe durch Diskriminierung (monetäre) Vorteile verschaffen können und sie daher Anreize für die Aufrechterhaltung dieses Zustandes besitzen.

\subsubsection{Monopsonistische Firmen auf dem Arbeitsmarkt}

Operieren auf dem Arbeitsmarkt lediglich ein einzelner oder eine geringe Anzahl kooperierender Arbeitgeber, die sich einer unorganisierten ausländischen Arbeitnehmerschaft gegenübersehen, dann können diese ihre Machtposition dazu benutzen, den ausländischen Arbeitnehmern einen geringeren Lohn als den Marktlohn zu diktieren, um dadurch ihre Gewinne zu erhöhen. ${ }^{156}$ Im Falle vollständiger Diskriminierung wird jeder Arbeitnehmer genau seinen Reservationslohn erhalten. Da zu vermuten ist, dass der Reservationslohn von Ausländern häufig unter jenem der Schweizer liegt, erhalten Ausländer bei gleicher Produktivität niedrigere Löhne. Für inländische Arbeitgeber besteht daher ein Anreiz, vor allem ausländische Arbeitnehmer einzustellen. ${ }^{157}$

Das Ausmass der Diskriminierung hängt dabei in erster Linie von zwei Faktoren ab. Erstens ist die Machtverteilung zwischen Arbeitnehmern und Arbeitgebern von grosser Bedeutung. Zweitens gilt es das Zusammenspiel zwischen der schweizerischen und der ausländischen Arbeitnehmerschaft zu berücksichtigen. Besteht bei Lohnverhandlungen ein hohes Mass an Kooperation zwischen in- und ausländischen Arbeitnehmern, dann verbleibt für die Arbeitgeberseite lediglich ein geringes Diskriminierungspotential. Dieses fällt umso grösser aus, je stärker es den Arbeitgebern gelingt, einen Keil zwischen die in- und ausländischen Arbeitnehmer zu treiben, so dass eine gemeinsame Interessenvertretung beider Gruppen erschwert oder verhindert wird. Dies wird auch dann der Fall sein, wenn es ihnen

155 Siehe KALLEBERG (1986).

156 Der Ansatz der monopsonistischen Diskriminierung geht auf ROBINSON (1933) zurück. Zwei Bedingungen müssen allerdings erfüllt sein, damit monopsonistische Diskriminierung stattfinden kann. Erstens muss sich das Arbeitsangebot in unterschiedliche Gruppen aufspalten lassen, z.B. entlang spezifischer individueller Merkmale wie dem Geschlecht oder der Nationalität. Zweitens müssen sich die Arbeitsangebotselastizitäten zwischen den beiden Gruppen unterscheiden [siehe auch KAUFMAN (1989)].

157 Siehe Velling (1995a). 
gelingt, die Unerfahrenheit ausländischer Arbeitskräfte auszunutzen. Schliesslich trifft dies auch zu, wenn sie in der Lage sind, gegenüber den ausländischen Arbeitnehmern ein Drohpotential aufzubauen, aufgrund der oftmals, institutionell bedingten, unsichereren Arbeitsmarktstellung der Ausländer. Diskriminierung kann sich für Arbeitnehmer in Beschäftigungsrückgängen und/oder Lohneinbussen äussern. Das in Art. 9 Abs. 1 BVO festgeschriebene Prinzip gleicher Lohnund Arbeitsbedingungen von In- und Ausländern impliziert jedoch, dass dieser Ansatz zur Erklärung von Diskriminierung in der Schweiz von untergeordneter Bedeutung sein dürfte.

\subsubsection{Gewerkschaften als Monopolisten}

Die Funktion von Gewerkschaften lässt sich nicht nur als Gegengewicht zur arbeitgeberseitigen Diskriminierung interpretieren, sondern kann selbst als Quelle von Lohn- bzw. Einkommensdiskriminierung gegenüber ausländischen Arbeitnehmern verstanden werden. ${ }^{158}$ Die Ausführungen in Abschnitt 6.3.2.2 haben gezeigt, dass Vorurteile von Arbeitnehmern keine hinreichende Bedingung für die längerfristige Existenz von Einkommensdiskriminierung darstellen. Durch die Bildung eines Arbeitsangebotsmonopols kann es den inländischen Arbeitnehmern jedoch gelingen, ihren Präferenzen Nachdruck zu verleihen, um einen über dem Marktlohn liegenden Lohn zu realisieren. Da es sich bei den inländischen Arbeitnehmern i.d.R. um die Majoritätengruppe handelt, ist sie in der Lage, ihren Forderungen genügend Gewicht zu verleihen. Gelingt es den Gewerkschaften, die Monopolrenten abzuschöpfen, dann werden die Gewerkschaftsmitglieder bestrebt sein, den Gewerkschaftszugang zu beschränken, um die Abschöpfung der Renten längerfristig zu sichern. Als Kriterien für die Implementierung von Zulassungsbeschränkungen können dabei die Diskriminierungspräferenzen dienen.

\subsubsection{Regierung als Monopolist}

Neben den Arbeitgebern und Arbeitnehmern geht auch von der Regierung ein Diskriminierungspotential aus, da diese in vielen Bereichen aufgrund ihrer hoheitlichen Rechte über ein bedeutend grösseres Machtpotential verfügt als Unternehmen oder Gewerkschaften. Zudem werden Regierungen i.d.R. nicht von Gewinnmaximierungsüberlegungen gelenkt, was dazu führt, dass die Gegenargumente gegen Diskriminierung, wie sie insbesondere in Abschnitt 6.3.1 angeführt wurden, hier nicht zum Tragen kommen. Wird von der Annahme ausgegangen, dass die Majoritätengruppe substantiellen Einfluss auf die Politik der Regierung ausübt, dann erwächst daraus ein Potential an regierungsseitiger Diskriminierung. Diese kann sich in Verschärfungen der Ausländergesetzgebung oder Beschrän-

158 Siehe CAIN (1986). 
kungen der Rechte und Möglichkeiten der Ausländer auf dem Arbeitsmarkt manifestieren.

Verschiedene Gründe sprechen dafür, dass die Regierung ihren hoheitlich-diskretionären Spielraum zugunsten der Inländer ausnützt. Erstens handelt es sich bei den Inländern um die Bevölkerungsmehrheit. Da die Regierung in einem demokratischen Staat eine politische Mehrheit benötigt, ist sie auf die Stimmen der Inländer angewiesen und trägt daher mit ihrer Politik vor allem den Anliegen dieser Bevölkerungsgruppe Rechnung. Zweitens werden diese Überlegungen durch die Tatsache untermauert, dass Ausländer i.a. kein Stimm- und Wahlrecht besitzen und daher von Politikern nicht in ihr Stimmenmaximierungskalkül miteinbezogen werden müssen.

Abschliessend gilt es noch, auf zwei Punkte hinzuweisen. Erstens basierten die bisherigen Ausführungen auf einer statischen Betrachtungsweise. Diskriminierung kann aber nicht nur zu Lohn- und Einkommensunterschieden zu einem bestimmten Zeitpunkt führen, sondern auch zu intertemporal wachsenden Einkommensdisparitäten, wenn Diskriminierung z.B. die Aufstiegschancen ausländischer Arbeitskräfte beeinträchtigt. ${ }^{159}$ Zweitens müssen nicht-produktivitätsbedingte Performanceunterschiede zwischen In- und Ausländern nicht notwendigerweise das Ergebnis von Diskriminierung sein, sondern können auch das Resultat freiwilliger Entscheidungen sein.

\subsection{ARBEITSMARKTSEGMENTATION}

Die bisherigen Überlegungen haben bereits Anhaltspunkte dafür geliefert, dass nicht nur Einkommensdiskriminierung als Ursache für die Existenz von Einkommensdifferentialen zwischen zwei Gruppen in Frage kommt, sondern dass diese auch durch die Segmentation des Arbeitsmarktes bedingt sein können. ${ }^{160}$ Die bisherigen Ausführungen dieses Kapitels haben gezeigt, dass die neoklassische Theorie die langfristige Existenz diskriminierender Lohndifferentiale sowie beruflicher Diskriminierung nur partiell und die in Realität häufiger bestehende branchenspezifische Diskriminierung nicht zu erklären vermag. Institutionelle Faktoren, wie die Zugangsbeschränkungen zu bestimmten Berufen, besitzen ebenfalls lediglich einen geringen Erklärungswert für die sektorielle und branchenspezifische Allokation ausländischer Erwerbstätiger. Die Theorie der Arbeitsmarktsegmentation

159 Siehe KAUFMAN (1989).

160 Die meisten Studien befassen sich in diesem Zusammenhang, analog zur Analyse der Lohnbzw. Einkommensdiskriminierung, mit ethnischer oder geschlechtsspezifischer Arbeitsmarktsegmentation [siehe CHARLES (1987), GiLl (1994), STEWART (1983), TERREL (1992)]. Mit der Diskriminierung zwischen In- und Ausländern im Rahmen der Arbeitsmarktsegmentationstheorie haben sich vor allem PIORE (1979), aber auch SEIFERT (1993) oder VELLING (1995a, 1995b) befasst. 
bildet daher einen komplementären Ansatz zur Erklärung langfristig existierender Ungleichgewichte auf dem Arbeitsmarkt. Im Rahmen dieses Ansatzes werden Arbeitslosigkeit bzw. Disparitäten in der Einkommensverteilung nicht auf die Existenz horizontaler Wettbewerbsbeschränkungen, sondern auf vertikale Mobilitätsbeschränkungen der Arbeitskräfte zurückgeführt. ${ }^{161}$

Der Begriff der Arbeitsmarktsegmentation wurde Ende der 60er Jahre durch die amerikanische Arbeitsmarktforschung geprägt. Als Ausgangspunkt diente die Beobachtung, dass sich Unterbeschäftigung und Arbeitslosigkeit in bestimmten Gruppen wie den Frauen, Jugendlichen oder ethnischen Minoritäten konzentrierte, wobei dieses Ergebnis weitgehend unabhängig von den individuellen Qualifikationsmerkmalen der einzelnen Wirtschaftssubjekte Gültigkeit besass. ${ }^{162}$ Segmentation wird in diesem Zusammenhang als "... Grad der relativ dauerhaften Aufspaltung des Arbeitsmarktes in institutionell mehr oder weniger verfestigte Teilarbeitsmärkte ..." verstanden. ${ }^{163}$ In den verschiedenen Theorien zur Arbeitsmarktsegmentation wird somit unterstellt, dass der Gesamtarbeitsmarkt in relativ undurchlässige Teilarbeitsmärkte zerfällt, zwischen denen die Mobilität der Arbeitskräfte beschränkt ist. Die verschiedenen Teilarbeitsmärkte sind nicht für alle Arbeitskräfte voll zugänglich und verfügen über unterschiedliche Allokationsmechanismen.

Die Theorien zur Arbeitsmarktsegmentation relativieren somit durch die Annahme voneinander getrennter Teilarbeitsmärkte die Bedeutung von Löhnen und Preisen, d.h. der typischen Allokationsmechanismen der neoklassischen Theorie. ${ }^{164} \mathrm{Im}$ Rahmen dieser alternativen Ansätze sind die Prozesse zwischen den einzelnen Teilarbeitsmärkten vor allem durch institutionelle Regelungen sowie arbeitgeberund arbeitnehmerseitige Verhaltensweisen bestimmt. Innerhalb der einzelnen Teilarbeitsmärkte ist demgegenüber davon auszugehen, dass die Funktionsweise durch die klassischen Allokationsmechanismen gewährleistet ist. Es wird dabei i.a. unterstellt, dass die Segmentation des Arbeitsmarktes nicht auf produktivitätsbezogene Faktoren zurückzuführen ist, sondern durch Diskriminierung bedingt ist. ${ }^{165}$ Zur Vereinfachung der Analyse wird dabei meist von einer dualen Arbeitsmarktstruktur ausgegangen.

Im Kern stellt die duale Arbeitsmarkttheorie, die auf DOERINGER/PIORE (1971) zurückgeht, eine Übertragung des Konzeptes der dualen Ökonomie von AvERITT

\footnotetext{
161 Siehe FENINGER (1988).

162 Siehe SEIFERT (1993).

163 SENGENBERGER (1978: 6).

164 Als Ausgangspunkt für die meisten Ansätze zur Segmentationstheorie dienen die Arbeiten von DOERINGER/PIORE (1971) sowie von KERR (1977).
}

Siehe TAUBMANN/WACHTER (1986). 
(1968) auf den Arbeitsmarkt dar. ${ }^{166}$ AvERrTT konnte für die USA eine Dualisierungstendenz des privatwirtschaftlichen Sektors in Wettbewerbsindustrien auf der einen und Monopolindustrien auf der anderen Seite feststellen. Der Ansatz der dualen Arbeitsmarkttheorie lässt sich wie folgt charakterisieren:

\section{Tab. 6.2: Theorie dualer Arbeitsmärkte}

1. Die Wirtschaft besteht aus einem Hochlohn- und einem Niedriglohnsektor. Das Verhalten der Wirtschaftssubjekte in diesen Sektoren erfordert unterschiedliche Erklärungsmuster.

2. Die Arbeitsproduktivität ist primär ein Merkmal von Arbeitsplätzen und keine individuelle Eigenschaft von Arbeitnehmern.

3. Arbeitnehmer im sekundären Sektor weisen ein instabiles Beschäftigungsverhalten auf, das durch häufige Wechsel von Arbeitsplätzen und häufige Arbeitslosigkeit charakterisiert ist.

Quelle: Abrahamsen/Kaplanek/Schips (1986)

Im Zentrum der dualen Arbeitsmarkttheorie von DOERINGER/PIORE (1971) steht die Aufspaltung des Arbeitsmarktes in ein primäres und ein sekundäres Segment, wobei diese beiden Bereiche als weitgehend voneinander getrennt anzusehen sind und institutionell, technologisch und ökonomisch unterschiedlich aufgebaut sind. Der gesamtwirtschaftliche Arbeitsmarkt zerfällt somit in eine Reihe interner und externer Arbeitsmärkte, die das primäre und sekundäre Segment des Arbeitsmarktes ausmachen. Es wird im weiteren unterstellt, dass die Mobilität der Arbeitskräfte zwischen den beiden Arbeitsmarktsegmenten beschränkt ist. Wie aus TAB. 6.2 hervorgeht, ist das primäre Segment durch hohe Löhne, gute Arbeitsbedingungen und Aufstiegschancen sowie stabile Beschäftigungsverhältnisse charakterisiert, während das sekundäre Segment durch schlecht bezahlte Arbeitsplätze, ungünstige Arbeitsbedingungen, geringe Aufstiegschancen sowie instabile Beschäftigungsverhältnisse gekennzeichnet ist.

Während im Rahmen der neoklassischen Arbeitsmarkttheorie eher von einer Unterscheidung zwischen guten und schlechten Arbeitskräften ausgegangen wird, steht bei der dualen Arbeitsmarkttheorie die Differenzierung zwischen guten und schlechten Arbeitsplätzen im Vordergrund. Die duale Arbeitsmarkttheorie stellt somit einen Versuch dar, auf makroökonomischer Ebene einen kausalen Zusammenhang zwischen der gesamtwirtschaftlichen Entwicklung sowie derjenigen am Arbeitsmarkt herzustellen, wobei die Analyse der Funktionsweise der Arbeitsmärkte, insbesondere der Allokations- und Remunerationsprozesse, aus mikroökonomischer Perspektive erfolgt. ${ }^{167}$

Gemäss den bisherigen Ausführungen ist somit davon auszugehen, dass ausländische Arbeitskräfte vorwiegend in saisonabhängigen Branchen sowie den unteren Hierarchieebenen beschäftigt sind. Die Existenz eines sekundären Segments bildet

166 Siehe DOERINGER/PIORE (1971) sowie PIORE $(1978,1979)$.

167 Siehe ABRAHAMSEN/KAPLANEK/SCHIPS (1986). 
somit die Voraussetzung für einen stabilen primären Bereich, da er konjunkturbedingte Schwankungen zu absorbieren vermag. Die Arbeitnehmer des sekundären Segments übernehmen so eine Art Konjunkturpufferfunktion. Der hohe Anteil ausländischer Erwerbstätiger im sekundären Segment, ermöglicht den Inländern damit eine vergleichsweise stabilere und besser bezahlte Beschäftigung im primären Segment. ${ }^{168}$

Abschliessend gilt es auf drei wichtige Punkte hinzuweisen. Erstens handelt es sich bei der dualen Arbeitsmarkttheorie nicht um die einzige Erklärungsmöglichkeit für die Existenz produktivitätsunabhängiger und persistenter Lohndifferentiale. Eine Erklärung hierfür kann auch durch die Effizienzlohntheorie gegeben werden. ${ }^{169}$ Zweitens ist darauf hinzuweisen, dass insbesondere im Rahmen der empirischen Analyse eine Unterscheidung zwischen den Tatbeständen der Einkommensdiskriminierung und der branchenspezifischen Segregation von Bedeutung ist. Findet diese Differenzierung nämlich nicht statt, kann eine Überschätzung des Ausmasses der Einkommensdiskriminierung oder der branchenspezifischen Segregation resultieren. ${ }^{170}$ Drittens ist zu erwähnen, dass die Inländer ein grosses Interesse an der Segmentation des Arbeitsmarktes besitzen, da sie dadurch nicht nur eine stabile und gut bezahlte Beschäftigung im primären Sektor erreichen können, sondern auch vor der Konkurrenz ähnlich qualifizierter ausländischer Arbeitskräfte geschützt sind. Die Ausländer besitzen somit im Rahmen des Ansatzes der dualen Arbeitsmarkttheorie eine weitgehend komplementäre Stellung zu den Inländern. ${ }^{171}$

\subsection{FAZIT}

Die Zielsetzung dieses Kapitels bestand darin, die Bedeutung sowie die Auswirkungen von Diskriminierung auf die Arbeitsmarktperformance der Einwanderer zu diskutieren. Aus TAB. 6.1 ging hervor, dass die Beantwortung dieser Frage von der zugrundeliegenden Art der Diskriminierung abhängt. Die zusammenfassende Darstellung in TAB. 6.3 unterscheidet erstens zwischen den zwei in diesem Kapi-

168 LANG/DICKENS (1992: 3) folgern daher, dass "... unemployment is concentrated among the types of workers who are disproportionately represented in the low-wage sector".

169 Siehe Franz (1991). Eine empirische Studie für die Schweiz findet sich in Ferro-Luzzl (1994).

170 Einige Autoren weisen in diesem Zusammenhang darauf hin, dass branchenspezifische Segregation nicht nur durch Diskriminierung bedingt sein muss, sondern auch das Ergebnis einer freiwilligen Entscheidung sein kann [siehe GrLL (1994)].

171 Siehe PISChKe/Velling (1994). Die Frage, ob In- und Ausländer auf dem Arbeitsmarkt in einer substitutionalen oder komplementären Beziehung zueinander stehen, hat zu einer grossen Zahl an theoretischen und empirischen Studien geführt. Eine gute Übersicht findet sich in BAUER/ZIMMERMANN (1995b), GREENWOOD (1994), GREENWOOD/HUNT (1994), GREENWOORD/MCDOWELL $(1986,1994)$ sowie VELLING (1995a). 
tel untersuchten Diskriminierungsursachen, Einkommensdiskriminierung und Arbeitsmarktsegmentation. Zweitens sind die Auswirkungen von Diskriminierung auf die Arbeitslosigkeit und Einkommenshöhe abgebildet. Es zeigt sich, dass Diskriminierung lediglich für die benachteiligte Gruppe automatisch eine Verschlechterung der Arbeitsmarktperformance impliziert. Für die nicht-diskriminierte Gruppe kann demgegenüber sowohl eine Verschlechterung als auch eine Verbesserung der Performance stattfinden.

Tab. 6.3: Folgen von Einkommensdiskriminierung und Arbeitsmarktsegmentierung für in- und ausländische Arbeitskräfte

\begin{tabular}{|c|c|c|c|c|}
\hline \multirow{2}{*}{$\begin{array}{l}\text { Diskriminierungs- } \\
\text { ursache }\end{array}$} & \multicolumn{2}{|c|}{ Diskriminierte Gruppe } & \multicolumn{2}{|c|}{ Nicht-diskriminierte Gruppe } \\
\hline & $\begin{array}{l}\text { Arbeitslosigkeit/ } \\
\text { Nicht-Erwerbstätigkeit }\end{array}$ & $\begin{array}{l}\text { Ein- } \\
\text { kommen }\end{array}$ & $\begin{array}{l}\text { Arbeitslosigkeit/ } \\
\text { Nicht-Erwerbstätigkeit }\end{array}$ & $\begin{array}{l}\text { Ein- } \\
\text { kommen }\end{array}$ \\
\hline \multirow{6}{*}{$\begin{array}{l}\text { Vorurteile: } \\
\text { - durch Arbeitgeber } \\
\text { - durch nicht-diskri- } \\
\text { minierte Arbeit- } \\
\text { nehmer } \\
\text {-durch Kunden } \\
\text { Statistische Diskri- } \\
\text { minierung }\end{array}$} & & & & \\
\hline & $(-)$ & - & $(+)$ & $\sim$ \\
\hline & $(-)$ & $\sim$ & $(+)$ & + \\
\hline & $(-)$ & - & $(+)$ & $\sim$ \\
\hline & - & $(-)$ & + & $\sim$ \\
\hline & & & & \\
\hline $\begin{array}{l}\text { Diskriminierung } \\
\text { aufgrund von }\end{array}$ & $+/ \sim$ & - & $\sim /-1+$ & $\sim 1-1+$ \\
\hline Marktmacht & - & - & + & + \\
\hline $\begin{array}{l}\text { Segmentation des } \\
\text { Arbeitsmarktes }\end{array}$ & & & & \\
\hline
\end{tabular}

Quelle: Eigene Darstellung nach VELLNG (1995a)

Anmerkungen: $+=$ günstig, $-=$ ungünstig, $\sim=$ neutral

Vorurteilsbedingte Diskriminierung zwischen in- und ausländischen Arbeitnehmern führt zu nicht-produktivitätsbedingten Lohn- und Einkommensdifferentialen. Geht die Diskriminierung von den Arbeitgebern oder Kunden aus, so kommt es zu einer Umverteilung zwischen der diskriminierten Arbeitnehmergruppe und den Arbeitgebern bzw. Kunden. Nur wenn die Vorurteile von anderen Arbeitnehmern ausgehen und diese daher für ihre Vorurteile kompensiert werden müssen, können sie höhere Einkommen realisieren. Falls Einkommensdiskriminierung aufgrund gesetzlicher oder tarifvertraglicher Beschränkungen nicht zulässig ist, besteht die Möglichkeit, dass Ausländer bei den Einstellungs- und Beschäftigungschancen, bei der Berufswahl oder auch den Arbeitsbedingungen und Aufstiegschancen diskriminierendem Verhalten ausgesetzt sein können, was z.B. in einer Erhöhung des Arbeitslosigkeitsrisikos der Ausländer zum Ausdruck kommen kann. 
Statistische Diskriminierung führt zu einer eindeutigen Bevorzugung der nichtdiskriminierten Gruppe, d.h. der Inländer und der Benachteiligung der diskriminierten Gruppe, d.h. der Ausländer. Für Ausländer resultiert somit ceteris paribus eine schlechtere Beschäftigungsperformance, die sich in höherer Arbeitslosigkeit oder geringerer Erwerbsbeteiligung manifestieren kann sowie allenfalls auch in geringeren Einkommen. Diese Ergebnisse entsprechen damit den Auswirkungen, die sich im Falle institutioneller Diskriminierung aufgrund des Inländervorrangs bzw. des Kontingentierungssystems ergeben. Im Zeitverlauf ist allerdings aufgrund von Lernprozessen mit einer Verringerung der statistischen Diskriminierung zu rechnen.

Die Folgen ungleicher Machtverteilung sind demgegenüber stark kontextabhängig. Beherrschen monopsonistische Unternehmen den Arbeitsmarkt, so ist mit Einkommensreduktionen zu rechnen. Es ist jedoch nicht klar, ob diese Einkommensreduktion nur die ausländischen oder auch die inländischen Arbeitskräfte trifft. Substantielle Lohn- und Einkommensverringerungen können aber gleichzeitig dazu führen, dass ausländische Arbeitskräfte für inländische Arbeitgeber attraktiv werden und daher bevorzugt eingestellt werden. Sehen sich die inländischen Arbeitgeber demgegenüber einer Monopolgewerkschaft gegenüber, welche die Interessen der inländischen Arbeitnehmer vertritt, so kann dies zu einer Verbesserung der Beschäftigungs- und Einkommensperformance der inländischen im Vergleich zu den ausländischen Arbeitskräften führen. Letztlich besteht auch die Möglichkeit regierungsseitiger Diskriminierung gegenüber den ausländischen Arbeitskräften. Diese kann sich in der Beschränkung der Rechte und Möglichkeiten der Ausländer auf dem schweizerischen Arbeitsmarkt manifestieren.

Arbeitsmarktsegmentation, d.h. die Aufspaltung des Arbeitsmarktes in ein primäres und ein sekundäres Segment, führt gemäss der Theorie dualer Arbeitsmärkte dazu, dass sich die ausländischen Erwerbstätigen aufgrund ihrer Positionierung im sekundären Segment sowohl instabileren Beschäftigungsverhältnissen als auch tieferen Einkommen gegenübersehen. Die Pufferfunktion des sekundären Segments impliziert damit für die inländischen Erwerbstätigen im primären Segment höhere Einkommen und stabilere Beschäftigungsverhältnisse. 


\section{FORMULIERUNG VON HYPOTHESEN}

Zum Abschluss dieses zweiten Teils erfolgt eine kurze Synthese der zentralen Ergebnisse des ersten und zweiten Teils. Diese Synthese erfolgt im Rahmen der Formulierung von Hypothesen, die als Grundlage für die empirische Analyse des dritten Teils dieser Studie dienen. Der empirische Teil befasst sich mit der Untersuchung der Beschäftigungs- und Einkommensperformance der Einwanderer. Im Zentrum des Interesses stehen dabei einerseits die Determinanten der Beschäftigungs- und der Einkommensperformance. Andererseits gilt es, die Ursachen von Performanceunterschieden zwischen In- und Ausländern zu erörtern. Von besonderem Interesse ist dabei die Frage, ob sich diese Unterschiede in der Arbeitsmarktperformance von In- und Ausländern auf produktivitätsbedingte Faktoren zurückführen lassen oder ob sie das Ergebnis von Diskriminierung gegenüber den ausländischen Erwerbstätigen sind.

Während im Rahmen der theoretischen Analyse des zweiten Teils auf eine Unterscheidung der Beschäftigungs- und Einkommensperformance aufgrund der in weiten Teilen übereinstimmenden theoretischen Grundlagen verzichtet wurde, erfolgt im dritten, empirischen Teil eine getrennte Analyse dieser beiden Untersuchungsgegenstände. Anhand zweier Beispiele soll illustriert werden, dass zwischen ihnen zwar grosse Interdependenzen bestehen, dass aber gleichzeitig gute Gründe für eine getrennte Analyse sprechen. Einerseits ist zu vermuten, dass z.B. die Konzentration der ausländischen Erwerbstätigen in den unteren Hierarchiestufen sowohl einen negativen Effekt auf die Beschäftigungs- als auch auf die Einkommensperformance besitzt. Dies impliziert, dass die ausländischen Erwerbstätigen unattraktivere und unsicherere Beschäftigungsverhältnisse aufweisen und damit verbunden im Mittel auch ein tieferes Einkommensniveau besitzen.

Andererseits ist eine Situation denkbar, in der ein Land aus wirtschaftspolitischen Erwägungen durch Eingriffe in die Tarif- und Steuerpolitik eine egalitäre Einkommensverteilung herbeiführt, so dass zwischen In- und Ausländern hinsichtlich der Einkommensperformance keine grossen Unterschiede bestehen. Es wäre jedoch falsch, daraus zu folgern, dass In- und Ausländer eine äquivalente Arbeitsmarktperformance aufweisen. Aufgrund der wirtschaftspolitischen Eingriffe in den Preismechanismus, müssen die Anpassungsprozesse auf dem Arbeitsmarkt über den Mengenmechanismus, d.h. die Beschäftigung, stattfinden. Wird unterstellt, dass die Inländer eine vergleichsweise höhere Produktivität aufweisen, ist davon auszugehen, dass die Arbeitslosenquote der Ausländer über jener der Inländer liegt. Erst die Berücksichtigung sowohl der Einkommens- als auch der Beschäftigungsperformance ermöglicht daher aussagekräftige Ergebnisse zur Arbeitsmarktperformance. 


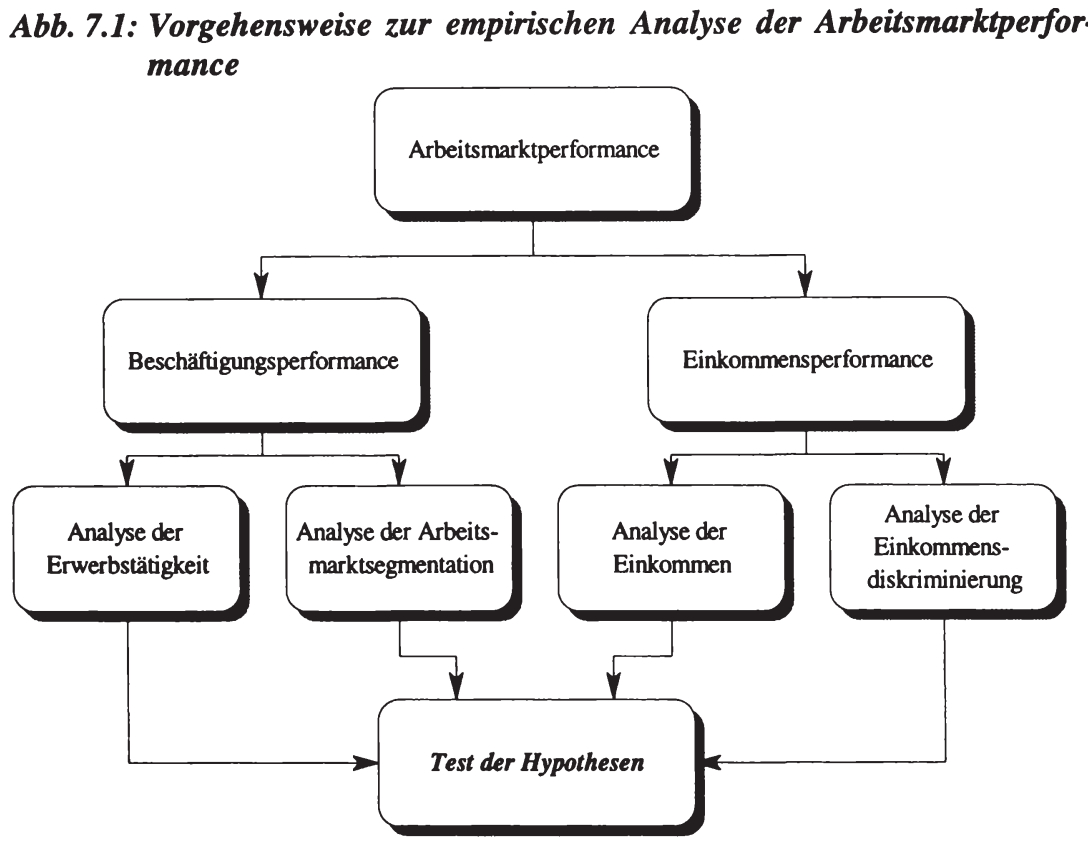

Quelle: Eigene Darstellung

Diese beiden Beispiele illustrieren, dass es sich bei den Untersuchungsgegenständen der Beschäftigungs- und der Einkommensperformance um komplementäre und interdependente Teilaspekte der Arbeitsmarktperformance handelt (siehe auch ABB. 7.1).

\subsection{FOLGERUNGEN AUS DER DESKRIPTIVEN UND DER THEORE- TISCHEN ANALYSE}

(i) Mit Blick auf die Zuwanderung in die Schweiz sind vor allem zwei Entwicklungen von Bedeutung. Erstens hat eine Verlagerung der Zuwanderung von den traditionellen Rekrutierungsgebieten, den Anrainerstaaten der Schweiz, zu den entfernteren und wirtschaftlich weniger entwickelten Regionen des peripheren europäischen bzw. des aussereuropäischen Raumes stattgefunden. Zweitens hat sich in den vergangenen Jahren mit der $\mathrm{Zu}$ nahme des Anteils der Familien- und Flüchtlingsmigration eine markante Verschiebung der Migrationsmotive vollzogen.

(ii) Die Ausführungen im Rahmen der theoretischen Analyse der Arbeitsmarktperformance haben gezeigt, dass die Selektivität der Zuwanderung wesent- 
lichen Einfluss auf die Arbeitsmarktperformance der Einwanderer besitzt. Die Selektivität wird vor allem durch die individuellen sozio-ökonomischen Charakteristika der Einwanderer bestimmt. Daneben wird sie aber u.a. auch von den Migrationsmotiven beeinflusst. Neben der Selektivität spielt, insbesondere bei der Betrachtung der intertemporalen Entwicklung der Arbeitsmarktperformance, d.h. der 'Arbeitsmarktassimilation' der Einwanderer, die internationale Transferierbarkeit des Humankapitals eine wichtige Rolle. Die Transferierbarkeit wird dabei durch die geographisch-kulturelle Distanz zwischen dem Herkunfts- und dem Zielland bestimmt. Diese Überlegungen verdeutlichen die Notwendigkeit einer nach Geschlecht und Nationalitätengruppen differenzierten Analyse, die der Heterogenität zwischen den einzelnen Wirtschaftssubjekten Rechnung trägt und damit verbunden, aussagekräftige Ergebnisse hinsichtlich der Beschäftigungs- und Einkommensperformance gewährleisten zu kann. Schliesslich hat die theoretische Analyse gezeigt, dass auch intervenierende Faktoren wie die Nachfrage nach ausländischen Arbeitskräften oder die rechtlichen Rahmenbedingungen der Migrationspolitik einen Einfluss auf die Performance besitzen.

(iii) Wie die theoretische Analyse jedoch ebenfalls gezeigt hat, ist davon auszugehen, dass neben diesen produktivitätsbedingten Faktoren auch diskriminierungsbedingte Faktoren einen Einfluss auf die Beschäftigungs- und Einkommensperformance besitzen. Zum einen werden sowohl die Beschäftigungs- als auch die Einkommensperformance von der Existenz bzw. dem Ausmass der Arbeitsmarktsegmentation beeinflusst. Der Begriff der Arbeitsmarktsegmentation steht im Rahmen der vorliegenden Studie sowohl für branchenspezifische als auch für hierarchische Segregation. Zum anderen wird die Einkommensperformance durch Einkommensdiskriminierung beeinflusst.

\subsection{HYPOTHESEN ZUR ARBEITSMARKTPERFORMANCE}

Zur Analyse der Arbeitsmarktperformance werden in einem ersten Schritt einige allgemeine Hypothesen formuliert, die sich sowohl auf die Beschäftigungs- als auch die Einkommensperformance beziehen. In einem zweiten Schritt folgen dann, jeweils einige auf die Beschäftigungs- und Einkommensperformance bezogene Hypothesen.

\subsubsection{ALLGEMEINE HYPOTHESEN}

(1) Die Ausstattung an beobachtbaren und nicht-beobachtbaren sozio-ökonomischen Charakteristika wie dem Humankapitalbestand oder dem Ehrgeiz, besitzt einen entscheidenden Einfluss auf die Arbeitsmarktperformance der Einwanderer. Es ist zu vermuten, dass interindividuell divergierende Eigenschaften zu geschlechts-, kohorten- und nationalitätenspezifischen Un- 
terschieden führen. Gemäss den theoretischen Überlegungen ist zu erwarten, dass die Einwanderer aus Nordeuropa das vorteilhafteste und die Einwanderer aus dem nicht-europäischen Raum das schlechteste Ausstattungsprofil aufweisen.

(2) Mit Blick auf die unvollständige internationale Transferierbarkeit von Humankapital ist davon auszugehen, dass die ausländischen Arbeitskräfte in einer ersten Phase nach der Zuwanderung eine vergleichsweise schlechte Arbeitsmarktperformance aufweisen. Im Zeitverlauf ist jedoch mit der Akkumulation ziellandspezifischen Humankapitals mit einer Verbesserung der Arbeitsmarktperformance, d.h. einer Arbeitsmarktassimilation, zu rechnen. Die grössten Anreize zu Investitionen in ziellandspezifisches Humankapital bestehen für diejenigen Einwanderer, deren anfänglicher Performancerückstand am ausgeprägtesten ausfällt, da sie die höchsten Ertragsraten besitzen.

(3) Je ausgeprägter das kulturelle und wirtschaftliche Gefälle zwischen dem Herkunfts- und Zielland ist, desto grössere Probleme bestehen bei der Transferierbarkeit des Humankapitals und umso grössere Unterschiede in der Arbeitsmarktperformance sind zwischen In- und Ausländern zu erwarten (nationalitätenspezifische Unterschiede). Aufgrund des vorteilhafteren Ausstattungsprofils sowie der besseren internationalen Transferierbarkeit des Humankapitals ist zu vermuten, dass Einwanderer aus Nordeuropa eine signifikant bessere Performance aufweisen als Einwanderer aus Südeuropa und dass diese wiederum erfolgreicher sein werden als Einwanderer aus NichtEuropa.

(4) In diesem Zusammenhang ist ebenfalls davon auszugehen, dass die verstärkte Zuwanderung aus nicht-europäischen Staaten in den letzten Jahrzehnten zu einer Verschlechterung der 'Qualität' und damit verbunden der Performance der Einwanderer geführt hat (kohortenspezifische Unterschiede). Für die jüngsten Zuwanderungskohorten ist aufgrund der Neuorientierung der schweizerischen Migrationspolitik seit Beginn der 90er Jahre allerdings mit einer partiellen Umkehr dieses Prozesses zu rechnen.

(5) Aufgrund der traditionellen Rollenverteilung zwischen Männern und Frauen sowie des damit verbundenen höheren Anteils an Familienmigration der Frauen bzw. Arbeitsmigration der Männer, ist mit einer vergleichsweise besseren Arbeitsmarktperformance der Männer zu rechnen (geschlechtsspezifische Unterschiede).

\subsubsection{SPEZIFISCHE HYPOTHESEN ZUR BESCHÄFTIGUNGSPERFORMANCE}

(6a) Die deskriptive Analyse hat gezeigt, dass Ausländer zwar eine höhere Erwerbsquote als Inländer aufweisen, dass sie aber gleichzeitig auch substantiell höhere Arbeitslosenquoten besitzen. Es ist zu vermuten, dass die vergleichsweise höheren Erwerbsquoten der Einwanderer vor allem auf die 
vorteilhaftere demographische Struktur der ausländischen Bevölkerung zurückzuführen sind. Unter Berücksichtigung dieses Sachverhalts sowie der sozio-ökonomischen Profile der einzelnen Wirtschaftssubjekte ist vor allem mit tieferen Erwerbsquoten der Frauen und signifikant höheren Erwerbslosenquoten der Einwanderer aus dem süd- und nicht-europäischen Raum zu rechnen.

(7a) Mit Blick auf die berufliche Stellung sowie die Beschäftigung nach Wirtschaftszweigen ist mit ähnlichen Ergebnissen zu rechnen. Es ist jedoch zu erwarten, dass sich die Beschäftigungsstruktur der ausländischen Erwerbstätigen mit zunehmender Aufenthaltsdauer an jene der inländischen Erwerbstätigen anpasst. Aufgrund der Ergebnisse der deskriptiven Analyse ist sogar anzunehmen, dass die Einwanderer aus Nordeuropa eine vorteilhaftere Beschäftigungsstruktur und daher eine bessere Beschäftigungsperformance besitzen als die schweizerischen Erwerbstätigen. In analoger Weise ist davon auszugehen, dass Männer eine vorteilhaftere Beschäftigungsstruktur aufweisen als Frauen.

(8a) Die Überlegungen zur Arbeitsmarktsegmentation geben in diesem Zusammenhang allerdings Anlass zur Vermutung, dass sich die nationalitäten- und geschlechtsspezifischen Unterschiede auch auf branchenspezifische und hierarchische Segregation zurückführen lassen. Es ist insbesondere damit zu rechnen, dass Frauen und Einwanderer aus dem süd- und nicht-europäischen Raum deshalb höhere Erwerbslosenquoten besitzen, weil sie mehrheitlich in strukturschwachen, unattraktiven Branchen sowie in unteren Hierarchiestufen beschäftigt sind, was mit instabileren Beschäftigungsverhältnissen und damit einem höheren Erwerbslosigkeitsrisiko verbunden ist.

\subsubsection{SPEZIFISCHE HYPOTHESEN ZUR EINKOMMENSPERFORMANCE}

(6b) Wird unterstellt, dass die anfänglichen Lohn- und Einkommensunterschiede zwischen In- und Ausländern vorwiegend auf Startschwierigkeiten im Zielland zurückzuführen sind, die durch die unvollständige internationale Transferierbarkeit des Humankapitals begründet sind, z.B. aufgrund von Sprachproblemen, dann ist zu erwarten, dass der Einkommensnachteil kurz nach der Einwanderung am stärksten ausfällt, dass er sich jedoch im Zeitverlauf im Rahmen der Einkommensassimilierung verringert. Es ist jedoch sowohl hinsichtlich der anfänglichen Unterschiede als auch des Konvergenzprozesses davon auszugehen, dass Einwanderer aus Nordeuropa die geringsten und Einwanderer aus Nicht-Europa die grössten anfänglichen Schwierigkeiten haben werden, so dass die Konvergenzrate der Nordeuropäer am geringsten und für die Nicht-Europäer am höchsten ausfallen wird. Gleichzeitig ist jedoch mit zunehmender Aufenthaltsdauer mit einem abnehmenden Assimilierungseffekt zu rechnen. 
(7b) Mit Blick auf die Einkommensassimilierung gilt es jedoch zu berücksichtigen, dass die intertemporale Entwicklung der Einkommen der Einwanderer nicht nur von Alters- bzw. Assimilierungseffekten, sondern auch von Kohorteneffekten beeinflusst wird, welche die Unterschiede in der 'Qualität' der verschiedenen Zuwanderungskohorten widerspiegeln. Analog zur Beschäftigungsperformance ist zu vermuten, dass bis auf die jüngsten Einwanderungskohorten der 90er Jahre eine Verschlechterung der Qualität der Einwanderer stattgefunden hat, die aus den strukturellen Veränderungen der Zuwanderung folgt.

(8b) Es ist jedoch, analog zur Beschäftigungsperformance, nicht auszuschliessen, dass die Unterschiede in der Einkommensperformance auf diskriminierungsbedingte Faktoren zurückzuführen sind. Einerseits können branchènspezifische und hierarchische Segregation dafür verantwortlich sein, dass Einkommensunterschiede zwischen In- und Ausländern längerfristig bestehen. Andererseits besteht aber auch die Möglichkeit, dass Einkommensdiskriminierung gegenüber Ausländern stattfindet, d.h. Ausländer mit vergleichbaren Fähigkeiten und vergleichbarer Stelle ein geringeres Einkommen erzielen als Inländer. 


\section{TEIL 3 (EMPIRIE): \\ EMPIRISCHE ANALYSE DER \\ ARBEITSMARKTPERFORMANCE}

\section{UNTERSUCHUNGSMETHODE UND EMPIRISCHE DATENBASIS}

Die theoretische Analyse hat gezeigt, dass die Arbeitsmarktperformance der ausländischen Arbeitskräfte von einer Reihe von Faktoren beeinflusst wird. Massgebliche Bedeutung kommt dabei nicht nur den individuellen beobachtbaren und nicht-beobachtbaren Charakteristika der einzelnen Wirtschaftssubjekte zu, sondern auch institutionellen Faktoren wie den rechtlichen Rahmenbedingungen. Letztlich ist auch auf die Möglichkeit diskriminierenden Verhaltens auf dem Arbeitsmarkt hinzuweisen. Die Vielzahl verschiedener Einflussfaktoren unterstreicht die Notwendigkeit einer differenzierten Vorgehensweise. Das primäre Ziel dieser Differenzierung besteht darin, die wichtigsten Unterschiede zu Schweizern aufzuzeigen, die sich aus der Ausländereigenschaft ergeben. Die schweizerische Bevölkerung bildet somit die Referenzgrösse für die Beurteilung der Arbeitsmarktperformance der ausländischen Arbeitskräfte.

Die Vorgehensweise in diesem Kapitel gestaltet sich dabei wie folgt. ${ }^{172}$ Im ersten Abschnitt wird auf die Abgrenzung zwischen nationalitäten- und einwanderungskohortenspezifischen Merkmalen eingegangen. Im zweiten Abschnitt wird die Bedeutung von Assimilierungs-, Kohorten-, Alters- und Periodeneffekten erläutert. Im dritten Abschnitt steht die Interaktion zwischen Nationalität und Einwanderungskohorte im Zentrum des Interesses. Im vierten Abschnitt wird das Datenset vorgestellt, das der empirischen Analyse zugrundeliegt. Im fünften Abschnitt folgen schliesslich einige abschliessende Bemerkungen.

Das Ziel dieses Kapitels ist es, eine Ausgangsbasis für die nachfolgende empirische Analyse zu schaffen, indem auf die Untersuchungsmethode eingegangen und die in der empirischen Analyse verwendete Datenbasis vorgestellt und kritisch diskutiert wird.

172 Die Ausführungen der ersten drei Abschnitte dieses Kapitels beziehen sich vor allem auf VELLING (1995a). 


\subsection{NATIONALITÄTEN- UND EINWANDERUNGSSPEZIFISCHE MERK- MALE}

Zwischen Einwanderern und Schweizern existieren zwei wesentliche Unterschiede. Erstens besitzen Einwanderer i.d.R. eine andere Nationalität, die Rückschlüsse über das Herkunftsland und die dort herrschenden ökonomischen, demographischen und politischen Bedingungen ermöglicht (nationalitätenspezifische Merkmale). Zweitens bestehen für Einwanderer aufgrund ihrer beschränkten Aufenthaltsdauer gewisse Nachteile. Diese können aus institutionellen Hemmnissen, mangelndem ziellandspezifischem Humankapital oder einem unterschiedlichen sozio-kulturellem Hintergrund erwachsen (einwanderungskohortenspezifische Merkmale). Es ist zu erwarten, dass sich diese Unterschiede im Rahmen eines Assimilierungsprozesses verringern.

\subsubsection{ABgRENZUNG VON NATIONALITÄTENGRUPPEN}

Zwischen Ausländern unterschiedlicher Nationalitäten bestehen eine Reihe von Unterschieden. Um eine ausreichende Zahl an Beobachtungen für die ökonometrische Analyse sicherzustellen sowie zur Gewährleistung der Übersichtlichkeit der Ergebnisse, ist es notwendig, die verschiedenen Herkunftsländer in Nationalitätengruppen zusammenzufassen. Zwei zentrale Punkte gilt es dabei zu berücksichtigen, um aussagekräftige empirische Ergebnisse zu gewährleisten. Einerseits sollte ein möglichst hoher Grad an Homogenität zwischen den Herkunftsländern innerhalb einer Nationalitätengruppe erreicht werden, andererseits sollten zwischen den Herkunftsländern in unterschiedlichen Nationalitätengruppen möglichst grosse Unterschiede existieren. Zur Beurteilung bzw. zur Konstruktion der verschiedenen Nationalitätengruppen werden in Anlehnung an VeLLING (1995a) folgende Unterscheidungsmerkmale berücksichtigt (siehe TAB. 8.1).

\section{Tab. 8.1: Unterscheidungsmerkmale zur Konstruktion von Nationalitäten-} gruppen

\begin{tabular}{|lc|}
\hline $\begin{array}{l}\text { 1. Institutionelle Rahmenbedingungen } \\
\text { 2. Grösse der Ausländerbevölkerung in }\end{array}$ & 4. Verhaltensspezifische Faktoren (insbe- \\
der Schweiz & - Integrationsbestreben \\
- Potential für Familienzusammenfüh- & - Partizipation am Arbeitsmarkt \\
rung & - Rückwanderungsabsichten \\
- Basis für Ketten- und Netzwerkmi- & - Familienzusammenführung \\
gration & 5. Entfernung zum Herkunftsland \\
3. Humankapitalspezifische Faktoren & - Kosten der Rückwanderung \\
- Kenntnisse einer der Landessprachen & - Kosten der Kommunikation und der \\
- durchschnittliche schulische Ausbil- & Kontaktaufnahme mit Personen im \\
dung & - Herkunftsland \\
- Transferierbarkeit von allgemeiner & 6. Situation im Herkunftsland \\
Ausbildung und beruflichen Qualifi- & - Kriegs- oder Bürgerkriegszustände \\
kationen & - Wohlstandsniveau/Armut \\
\hline
\end{tabular}

Quelle: Eigene Darstellung nach VELLNG (1995a) 
Auf der Grundlage dieser Kriterien sowie unter Berücksichtigung der in der SAKE verwendeten Nationalitäten werden drei Nationalitätengruppen definiert (siehe TAB. 8.2). Eine zweite detailliertere Definition der verschiedenen Nationalitätengruppen findet sich in TAB. A8.2 in Anhang 1. Während im Text sowohl in der deskriptiven als auch in der empirischen Analyse auf die erste Definition abgestellt wird, werden in Anhang 1 zusätzlich die Ergebnisse der deskriptiven Analyse für die zweite Definition ausgewiesen. Diese Vorgehensweise wird einerseits gewählt, um die Übersichtlichkeit der deskriptiven Analyse zu gewährleisten, sowie andererseits, um der teilweise geringen Zahl der Beobachtungen für die einzelnen Nationalitäten in der empirischen Analyse Rechnung zu tragen.

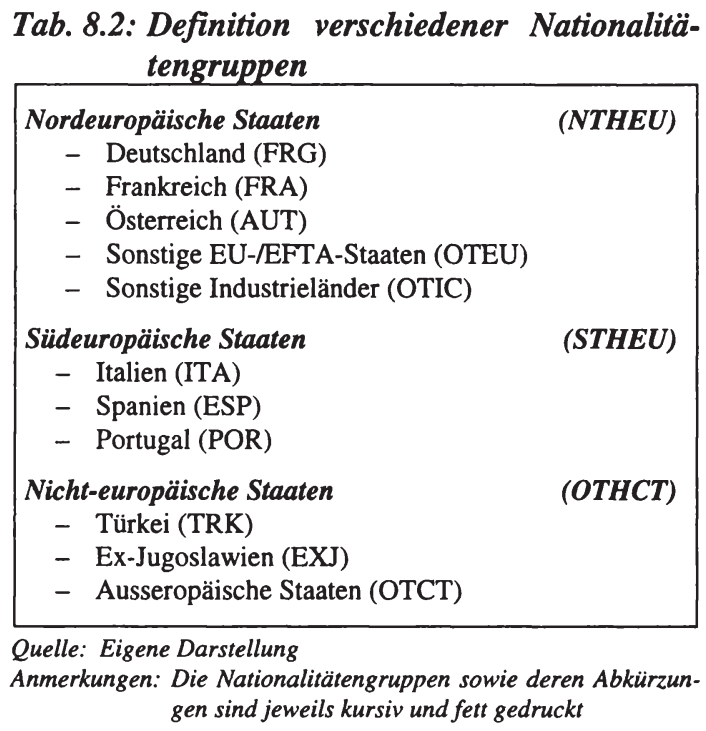

Die Verwendung dieser weniger differenzierten Definition ist allerdings mit Heterogenitätsproblemen behaftet. Dies trifft insbesondere für die Nationalitätengruppe OTCT zu, in der eine Vielzahl verschiedener Nationalitäten mit teilweise sehr unterschiedlichen sozio-ökonomischen Charakteristika und sehr geringen Fallzahlen zusammengefasst sind. Die empirischen Ergebnisse für diese Nationalitätengruppe sind daher mit Vorsicht zu interpretieren.

\subsubsection{ABgRENZUNG VON EINWANDERUNGSKOHORTEN}

Im Gegensatz zur Bildung von Nationalitätengruppen gestaltet sich die Differenzierung der Einwanderer nach Einwanderungskohorten wesentlich einfacher. Als Kriterium zur Definition verschiedener Einwanderungskohorten dient die Aufenthaltsdauer gemessen in Jahren. Es stellt sich in diesem Zusammenhang allerdings 
die Frage, wie die in der Schweiz geborenen bzw. aufgewachsenen Ausländer, d.h. die Ausländer der Zweiten Generation, zu behandeln sind. ${ }^{173}$ Die Ausführungen des theoretischen Teils haben gezeigt, dass aus ökonomischer Sicht einige Argumente dafür sprechen, dass zwischen Einwanderern der Ersten und der Zweiten Generation signifikante Unterschiede in der Arbeitsmarktperformance bestehen. Diese Schlussfolgerung beruht auf der Erkenntnis, dass Einwanderer der Zweiten Generation i.d.R. besser mit der Sprache der jeweiligen Region vertraut sind und zumindest teilweise das schweizerische Schulsystem durchlaufen haben. Es ist daher zu vermuten, dass sie über einen höheren Anteil an ziellandspezifischem Humankapital verfügen und daher besser in die schweizerische Gesellschaft integriert sind als Einwanderer der Ersten Generation. Um Heterogenitätsprobleme zu vermeiden, wird daher im Rahmen der vorliegenden Studie lediglich die Performance von Einwanderern der Ersten Generation untersucht.

Aus TAB. 8.3 geht hervor, dass in Abhängigkeit der Aufenthaltsdauer sechs verschiedene Einwanderungskohorten unterschieden werden. Die Abgrenzung zwischen den verschiedenen Einwanderungskohorten ergibt sich dabei aus den gesetzlichen Bestimmungen der schweizerischen Ausländerpolitik und trägt so der mit zunehmender Aufenthaltsdauer stattfindenden Assimilation der Einwanderer Rechnung. Dies lässt sich anhand der Erteilung von Niederlassungsbewilligungen illustrieren, die je nach Staatsangehörigkeit nach einer Aufenthaltsdauer von 5 bis 10 Jahren erfolgt. Für die Erlangung der schweizerischen Staatsangehörigkeit ist demgegenüber i.d.R. eine Mindestaufenthaltsdauer von 15 Jahren notwendig.

\section{Tab. 8.3: Definition verschiedener Einwanderungskohorten}

\begin{tabular}{|lrr|}
\hline \multicolumn{2}{|l|}{ Einwanderungskohorten (Bezugsjahr 1995) } & \\
- Erste Kohorte: Ausländer, die vor weniger als 1 Jahr eingewandert sind & $(<1)$ \\
- Zweite Kohorte: Ausländer, die vor 1 bis 5 Jahren eingewandert sind & $(1-5)$ \\
- Dritte Kohorte: Ausländer, die vor 5 bis 10 Jahren eingewandert sind & $(5-10)$ \\
- Vierte Kohorte: Ausländer, die vor 10 bis 15 Jahren eingewandert sind & $(10-15)$ \\
- Fünfte Kohorte: Ausländer, die vor 15 bis 20 Jahren eingewandert sind & $(15-20)$ \\
- Sechste Kohorte: Ausländer, die vor mehr als 20 Jahren eingewandert sind & $(>20)$ \\
\hline
\end{tabular}

Quelle: Eigene Darstellung

Anmerkungen: Die Abkürzungen der Einwanderungskohorten sind jeweils kursiv und fett gedruckt

Die Unterscheidung von Einwanderungskohorten ist von Bedeutung, da sich so eine Vermischung von Assimilierungs-, Kohorten- und Alterseffekten und damit eine Verzerrung der empirischen Ergebnisse vermeiden lässt. Der Stellenwert der

173 Unter Einwanderern der Zweiten Generation werden alle ausländischen Staatsangehörigen subsumiert, die bis zum 16. Lebensjahr in die Schweiz eingewandert sind oder in der Schweiz geboren und aufgewachsen sind. 
Interaktion zwischen Nationalitätengruppen und Einwanderungskohorten wird in Abschnitt 8.3 erläutert.

\subsection{Assimilierungs-; Kohorten-, Alters- und Perioden- EFFEKTE}

Die empirische Analyse dieser Studie beruht auf den Daten der Schweizerischen Arbeitskräfteerhebung (SAKE) des Jahres 1995, wobei für die Analyse der Arbeitsmarktsegmentation auch die Daten des Jahres 1991 verwendet werden. Anhand des Bezugsjahres 1995 lassen sich einige wichtige Zusammenhänge zwischen Assimilierungs-, Kohorten-, Alters- und Periodeneffekten illustrieren. Daraus lassen sich unmittelbar zwei Informationen ableiten. Erstens resultiert die Aufenthaltsdauer der Einwanderer, die als Indikator für die institutionelle Verfestigung des Aufenthalts- und Arbeitsmarktstatus dient und damit verbunden der Assimilierung (Effekt der Aufenthaltsdauer). Zweitens kann aus der folgenden Identitätsbeziehung das Zuzugsjahr der Zuwanderer bestimmt werden

(8.1) Aufenthaltsdauer $=t_{95}-t_{\text {Einreise }}$.

Aus dem Einwanderungsjahr lassen sich gleichzeitig Informationen über die $\mathrm{Zu}-$ sammensetzung des Zuwanderungsstromes zu diesem Zeitpunkt gewinnen. Insbesondere ergeben sich daraus Hinweise auf die zu jener Zeit vorherrschenden wirtschaftlichen und politischen Rahmenbedingungen, die zu einer bestimmten Konstellation bzw. Zusammensetzung der Zuwanderung geführt haben (Kohorteneffekt). Für die Schweiz folgt daraus, dass die seit mehr als 20 Jahren in der Schweiz lebenden Ausländern fast ausschliesslich ehemalige Gastarbeiter sind, während jüngere Einwanderungskohorten zu einem substantiellen Teil aus nachgezogenen Familienangehörigen sowie aus Flüchtlingen und Asylbewerbern bestehen. Wie in den Kapiteln 2 und 3 bereits betont wurde, hat im Zeitverlauf zudem eine Verschiebung hinsichtlich der Herkunftsländer stattgefunden. Im Gegensatz zu diesen beiden Effekten, ist der Alterseffekt, der die Auswirkungen von Altersunterschieden bei der Einreise misst, nur mittelbar mit der Aufenthaltsdauer verknüpft. Es gilt

$$
\text { Alter }_{95}=\text { Aufenthaltsdauer }+ \text { Alter }_{\text {Einreise }} \text {. }
$$

Die Wirkungsweise des Alterseffektes hängt im einzelnen vom Zusammenspiel der Aufenthaltsdauer und dem Alter bei der Einreise ab. Aus TAB. 8.6 geht hervor, dass mit Ausnahme der beiden jüngsten Einwanderungskohorten keine allzu grossen Unterschiede hinsichtlich des Medianalters bestehen. ${ }^{174}$ Das Alter bei der Einreise in Gl. (8.2) variiert, auf den Median bezogen, nur geringfügig in Abhängig-

174 Der Vorteil des Medians, im Vergleich zum Durchschnitt, besteht darin, dass der Median von extremen Werten unbeeinflusst bleibt und daher stärker als der Durchschnitt die Mitte einer Verteilung widerspiegelt. 
keit der betrachteten Einwanderungskohorte. Für weibliche Einwanderer liegt es zwischen 23 und 26 Jahren, für männliche Einwanderer zwischen 22 und 24 Jahren. Aus diesem Grund erscheint eine Differenzierung des Einreisealters nach Einwanderungskohorten nicht notwendig, da das Medianalter im wesentlichen die Aufenthaltsdauer widerspiegelt. Für die jüngste Einwanderungskohorte bedeutet dies 34 Jahre, für die älteste Kohorte 53 Jahre.

Aus der Vermischung von Kohorten-, Alters- bzw. Assimilationseffekten ${ }^{175}$ sowie Periodeneffekten ${ }^{176}$ bei der Analyse der Einkommensentwicklung im Rahmen von Querschnittsuntersuchungen ergibt sich ein Problem, das jedoch durch die Verwendung von Längsschnittinformationen, etwa in Form eines Paneldatensatzes oder über das sog. 'poolen' von Daten zweier Querschnitte (Pseudo-Panel), entschärft werden kann. ${ }^{177}$ Die angesprochene Problematik, die vor allem für die Analyse der Einkommensperformance der Einwanderer in Kapitel 10 von Bedeutung ist, lässt sich anhand der folgenden Überlegungen veranschaulichen. ${ }^{178}$

\section{Tab. 8.4: Zentrale Ergebnisse der Analyse von CHISWICK}

1. Das Alters-Einkommens-Profil fällt für Einwanderer steiler aus als für Einheimische
Dieses Ergebnis lässt sich im Rahmen der Humankapitaltheorie damit erklären, dass die
Investitionsanreize für Einwanderer wegen des anfänglich höheren Ertrages grösser sind
und ihre Einkommen daher stärker wachsen als jene der Einheimischen.
2. Das Alters-Einkommens-Profil der Einwanderer übertrifft dasjenige der Einheimi-
schen nach einem Aufenthaltszeitraum von ca. 10 - 15 Jahren
Dieses Ergebnis lässt sich darauf zurückführen, dass die Einwanderer eine positive Se-
lektion aus der Gesamtbevölkerung des Herkunftslandes darstellen und den Einheimi-
schen im Zielland, aufgrund von nicht-beobachtbaren Charakteristika wie der Motiva-
tion und dem Ehrgeiz, überlegen sind.

Quelle: Eigene Darstellung

Als Ausgangspunkt dient die bereits mehrfach erwähnte Studie von CHISwICK (1978), in der die Einkommensdisparitäten bzw. -entwicklung zwischen Einwan-

175 Aufgrund der vorangegangenen Ausführungen werden im folgenden der Assimilations- und der Alterseffekt zum 'Alterseffekt' zusammengefasst. Sie entsprechen damit dem in der Literatur verwendeten sog. 'ageing effect'. Dieser aggregierte Effekt misst den Einfluss zunehmenden Alters sowie zunehmender Aufenthaltsdauer auf die intertemporale Entwicklung der Arbeitsmarktperformance.

176 Periodeneffekte spiegeln die Auswirkungen konjunktureller und struktureller Veränderungen auf die Performance der In- und Ausländer wider.

177 Von Paneldaten spricht man, wenn für eine bestimmte Untersuchungseinheit in mehreren aufeinanderfolgenden Zeitpunkten Beobachtungen für dasselbe Merkmal vorliegen. Ein guter Überblick zu dieser Thematik sowie zur entsprechenden Vorgehensweise findet sich in BORJAS (1989a, 1991b, 1994b), LICHT/STEINER (1993) sowie PISCHKE (1992).

Siehe auch WEBER (1993). 
derern und Einheimischen anhand von Querschnittsdaten analysiert wurden. Auf der Grundlage des in Kapitel 4 behandelten Humankapitalansatzes untersuchte CHISWICK im Rahmen von Querschnittsanalysen und mit Hilfe linearer Regressionsmodelle den Einfluss einer Reihe sozio-ökonomischer Merkmale auf das Einkommensniveau. CHISWICK kam im Rahmen dieser Studie zu den in TAB. 8.4 dargestellten zentralen Ergebnissen:

Diese Vorgehensweise hat in der Literatur eine kritische Diskussion hinsichtlich der Probleme bei der Verwendung von Querschnittsdaten bei der Analyse dynamischer Fragestellungen, wie der intertemporalen Einkommensentwicklung, ausgelöst. Vor allem BORJAS (1985, 1987, 1989a, 1990, 1991b, 1994b) hat in einer Reihe von Studien auf die mit einer solchen Vorgehensweise verbundenen Probleme hingewiesen. Das grundlegende Problem im Rahmen der Analyse von Querschnittsdaten besteht darin, dass sich Einkommensunterschiede zwischen In- und Ausländern auf vier unterschiedliche Effekte zurückführen lassen:

Tab. 8.5: Determinanten der Einkommensunterschiede zwischen Einwanderern und Einheimischen

\begin{tabular}{|lll}
\hline 1. & $\begin{array}{l}\text { Einkommensunterschiede sind durch Altersunter- } \\
\text { schiede zwischen In- und Ausländern bedingt }\end{array}$ & Alterseffekt \\
2. & $\begin{array}{l}\text { Einkommensunterschiede sind durch Unterschiede } \\
\text { zwischen Einwanderungskohorten bedingt }\end{array}$ & Kohorteneffekt \\
2. & $\begin{array}{l}\text { Einkommensunterschiede sind durch Unterschiede in } \\
\text { den Effekten von Veränderungen im wirtschaftlichen }\end{array}$ & Perioden- oder Zeiteffekt \\
Umfeld bedingt & \\
4. & $\begin{array}{l}\text { Einkommensunterschiede sind durch Diskriminierung } \\
\text { auf dem Arbeitsmarkt bedingt }\end{array}$ & Diskriminierungseffekt
\end{tabular}

Quelle: Eigene Darstellung

BORJAS bezieht sich in seiner Kritik in erster Linie darauf, dass im Rahmen der Analyse von Querschnittsdaten eine Unterscheidung zwischen Alters- und Kohorteneffekten nicht möglich ist. Er gelangt dabei zum Schluss, dass die Aussagen zur Einkommensentwicklung der Einwanderer zu relativieren sind und sich der Anpassungs- bzw. Assimilationsprozess in den Vereinigten Staaten über einen weit längeren Zeitraum erstreckt, als die Analyse von CHISwICK impliziert. Die empirischen Ergebnisse von BORJAS weisen sogar darauf hin, dass im Zeitverlauf eine Verschlechterung der 'Qualität' der Einwanderungskohorten stattgefunden hat. Diese Zusammenhänge lassen sich anhand der folgenden Graphik veranschaulichen. ${ }^{179}$

179 Siehe auch BORJAS (1989a). 


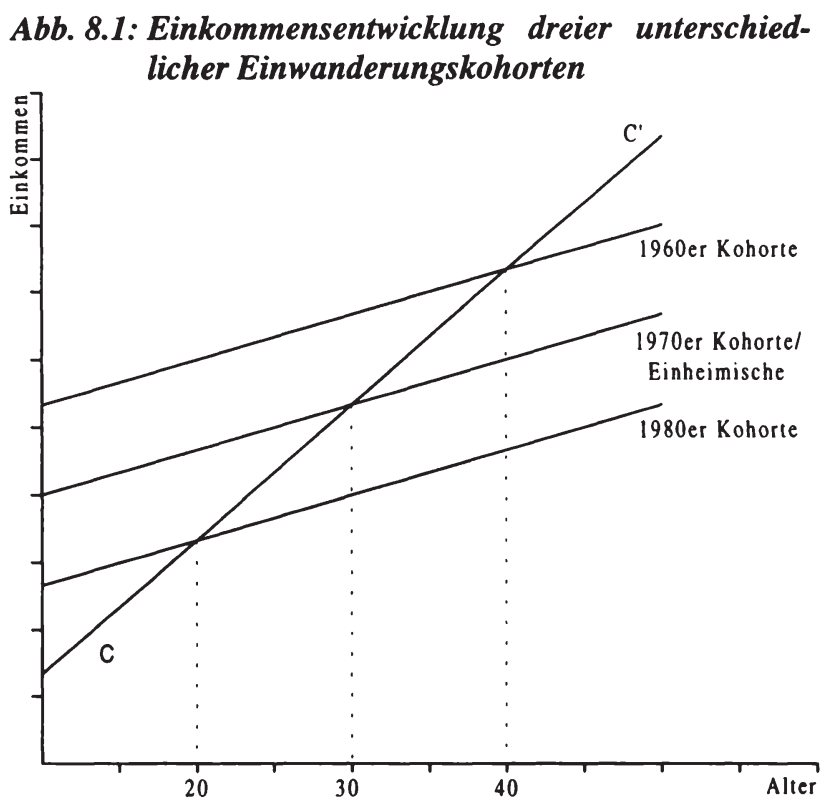

Quelle: Eigene Darstellung nach BORJAS (1985)

Aus ABB. 8.1 geht hervor, dass die 1960er Einwanderungskohorte am produktivsten ist und daher, im Vergleich zu den Einwanderungskohorten der Jahre 1970 und 1980, sowohl das höchste Einkommensniveau als auch das vorteilhafteste Alters-Einkommensprofil aufweist. In ABB. 8.1 ist somit eine intertemporale Verschlechterung der 'Qualität' der Einwanderer erkennbar. Wird nun unterstellt, dass die einheimischen Arbeitnehmer dieselben arbeitsmarktrelevanten Charakteristika aufweisen wie die Einwanderer der 1970er Kohorte, so fallen die beiden AltersEinkommensprofile, wie in ABB. 8.1 dargestellt, zusammen. Die Parallelität der Alters-Einkommensprofile impliziert, dass weder zwischen den einzelnen Einwanderungskohorten noch zwischen den Einheimischen und Einwanderern eine Assimilation im Sinne einer Angleichung der Einkommen stattfindet.

Anhand von ABB. 8.1 lässt sich veranschaulichen, dass die Verwendung von Querschnittsdaten bei der Analyse der Einkommensperformance, zu verzerrten Ergebnissen führen kann. Wird eine Einkommensregression auf der Basis der Daten des Jahres 1980 geschätzt, so gehen die Einkommen verschiedener Kohorten in diese Schätzung ein. Fälschlicherweise werden die 20-jährigen der 1980er Kohorte, die 30-jährigen der 1970er Kohorte sowie die 40-jährigen der 1960er Kohorte direkt miteinander verglichen, was in unserem Beispiel dazu führt, dass aus der Querschnittsanalyse ein steileres Einkommensprofil (Gerade $\mathrm{CC}^{\prime}$ ) resultiert als aus der Längsschnittsanalyse, in der zusätzlich kohortenspezifische Infor- 
mationen berücksichtigt werden. BoRJAS kommt daher zum Schluss, dass die optimistischen Ergebnisse der Analyse von CHISWICK vor allem auf die Vermischung von Alters- und Kohorteneffekten zurückzuführen sind. ${ }^{180}$

Zusammenfassend lässt sich somit festhalten, dass sich die mit der Analyse von Querschnittsdaten zusammenhängenden Probleme bzw. Verzerrungen der empirischen Ergebnisse, aufgrund kohortenspezifischer Effekte, durch die Verwendung von Längsschnittsinformationen vermeiden lassen. Wie bereits erwähnt wurde, kann diesem Umstand durch die Verwendung von Paneldaten Rechnung getragen werden. Die Analyse der vorliegenden Studie beschränkt sich allerdings auf die Analyse von Querschnittsdaten (siehe auch Kapitel 11).

\subsection{INTERAKTION ZWISCHEN NATIONALITÄT UND EINWANDE- RUNGSKOHORTE}

Die Interaktion zwischen Nationalitäten und Einwanderungskohorten geht unmittelbar aus den vorangegangenen Ausführungen hervor, da sich jede Kohorte aus Einwanderern verschiedener Nationalitäten zusammensetzt. Nationalitäten, die in einer bestimmten Einwanderungskohorte stark vertreten sind, besitzen demgemäss grossen Einfluss auf die sozio-ökonomischen und sozio-demographischen Merkmale der jeweiligen Kohorte.

Die im empirischen Teil gewählte Darstellungsweise für die deskriptiven Tabellen in den einzelnen Kapiteln verdeutlicht diesen Zusammenhang, da die Ausländer einer Nationalitätengruppe bzw. Einwanderungskohorte i.d.R. anteilsmässig mit einem bestimmten, gerade betrachteten Merkmal ausgewiesen werden (z.B. Erwerbstätige Ausländer nach Wirtschaftszweigen). Für eine bestimmte Nationalitätengruppe oder Einwanderungskohorte entspricht dabei der Anteil einer Merkmalsausprägung der Wahrscheinlichkeit, dass ein Mitglied dieser Nationalitätengruppe oder Einwanderungskohorte dieses Merkmal aufweist.

Zur Illustration kann der Fall eines Herkunftslandes wie Italien herangezogen werden, wobei unterstellt wird, dass die 'Qualität' einer Einwanderungskohorte jeweils dem Bevölkerungsdurchschnitt des Herkunftslandes entspricht. In Italien hat sich das durchschnittliche Bildungsniveau über die Jahre hinweg signifikant

180 Die Existenz von Kohorteneffekten kann durch eine Reihe von Ursachen bedingt sein (siehe auch Kapitel 6). Erstens kann die Veränderung der Rahmenbedingungen (rechtliche Rahmenbedingungen, wirtschaftliches und politisches Umfeld) auf dem 'Migrationsmarkt' Veränderungen in der Zusammensetzung des Zuwanderungsstromes zur Folge haben. Zweitens kann eine selektive Rückwanderung dazu führen, dass sich ein künstlicher positiver oder negativer Alterseffekt herausbildet. Drittens besitzt auch die zum Zeitpunkt der Einwanderung herrschende Wirtschaftslage im Zielland eine grosse Bedeutung. Viertens gilt es die Zahl der zum Zeitpunkt der Einwanderung gleichzeitig auf den Arbeitsmarkt eintretenden einheimischen Arbeitskräfte zu berücksichtigen [siehe BORJAS (1989b) und GREENWOOD/MCDOWELL (1986)]. 
verbessert. Da ein Grossteil der Zuwanderung aus Italien vor längerer Zeit erfolgte und daher der Anteil an Italienern mit einer langen Aufenthaltsdauer überproportional hoch ist, liegt das durchschnittliche Bildungsniveau der in der Schweiz lebenden italienischen Bevölkerung unter dem vergleichbaren Niveau in Italien. Es wäre somit zwar richtig, die in der Schweiz lebenden Italiener als vergleichsweise unqualifiziert zu bezeichnen, gleichzeitig aber falsch, daraus Schlussfolgerungen über das heutige Bildungsniveau in Italien und, damit verbunden, über die 'Qualität' jüngerer Einwanderungskohorten aus Italien zu ziehen.

Um daher eine aussagekräftige Darstellung und korrekte Interpretation der jeweiligen Anteile der Merkmalsausprägungen zu gewährleisten, findet im Rahmen der deskriptiven Analyse des empirischen Teils eine Differenzierung nach Nationalitätengruppen und Einwanderungskohorten statt. Gleichzeitig wird auch geschlechtsspezifischen Unterschieden Rechnung getragen, indem für Männer und Frauen eine getrennte Analyse durchgeführt wird.

\section{Tab. 8.6: Einwanderungskohorten nach Nationalitätengruppen (in Prozent)}

\begin{tabular}{|c|c|c|c|c|c|c|c|}
\hline \multirow[t]{2}{*}{$\begin{array}{l}\text { Nationalitäten- } \\
\text { gruppen }\end{array}$} & \multicolumn{6}{|c|}{$\begin{array}{c}\text { Einwanderungskohorten } \\
\text { (Aufenthaltsdauer in Jahren) }\end{array}$} & \multirow[t]{2}{*}{$\begin{array}{c}\text { Ausländer } \\
\text { (Total) }\end{array}$} \\
\hline & $<1$ & $1-5$ & $5-10$ & $10-15$ & $15-20$ & $>20$ & \\
\hline \multicolumn{8}{|l|}{ Männer } \\
\hline Nordeuropa & 45.7 & 58.1 & 32.2 & 18.5 & 21.6 & 33.7 & 31.8 \\
\hline Südeuropa & 39.2 & 16.8 & 33.6 & 47.8 & 56.6 & 59.4 & 48.8 \\
\hline Nicht-Europa & 15.0 & 25.1 & 34.3 & 33.6 & 21.8 & 6.8 & 19.4 \\
\hline Alter bei Einreise & 33 & 29 & 24 & 24 & 24 & 22 & 24 \\
\hline Derzeitiges Alter & 34 & 32 & 32 & 37 & 41 & 53 & 44 \\
\hline \multicolumn{8}{|l|}{ Frauen } \\
\hline Nordeuropa & 79.8 & 42.1 & 30.0 & 23.6 & 21.3 & 36.3 & 34.0 \\
\hline Südeuropa & 18.6 & 27.7 & 43.8 & 42.9 & 55.4 & 54.2 & 46.1 \\
\hline Nicht-Europa & 1.6 & 30.2 & 26.2 & 33.5 & 23.3 & 9.6 & 19.9 \\
\hline Alter bei Einreise & 27 & 26 & 26 & 23 & 22 & 23 & 24 \\
\hline Derzeitiges Alter & 27 & 29 & 33 & 36 & 39 & 53 & 41 \\
\hline
\end{tabular}

Quelle: SAKE (1995), eigene Berechnungen

Anmerkungen: Zur Aufschlüsselung der Nationalitätengruppen siehe TAB. 8.2

Aus TAB. 8.6 lassen sich vier wesentliche Aussagen ableiten. Erstens ist ersichtlich, dass das Gewicht der traditionellen Gastarbeiterländer Südeuropas (vor allem Italiens und Spaniens) zunimmt, je älter die Einwanderungskohorten sind. Dieser Sachverhalt lässt sich auch für andere westeuropäische Staaten beobachten, die Teil der ersten Einwanderungswelle der Nachkriegszeit waren. Allein aus Italien, Spanien und Deutschland stammen rund 75 Prozent aller Ausländer, die seit mehr 
als 20 Jahren in der Schweiz leben. ${ }^{181}$ Zweitens hat im Zeitverlauf eine Verlagerung hinsichtlich der Herkunftsländer stattgefunden. Über den Zeitraum 1975/80 bis 1985/90 erfolgte in einer ersten Phase eine Verlagerung der Zuwanderung innerhalb des suideuropäischen Raums nach Portugal sowie später in den nicht-europäischen Raum, insbesondere nach Ex-Jugoslawien und in die Türkei. ${ }^{182}$ In einer zweiten Phase, seit Ende der 80er Jahre, hat demgegenüber im Rahmen einer Trendwende eine starke Zunahme der Zuwanderung aus Nordeuropa, insbesondere aus Deutschland und Frankreich stattgefunden. ${ }^{183}$

Drittens spiegelt die Zunahme des Bestandes an Ausländern aus nicht-europäischen Staaten nicht nur die Verlagerung in den Rekrutierungsgebieten, sondern auch einen markanten Anstieg der Flüchtlingsmigration in die Schweiz wider. Viertens geht aus TAB. 8.6 hervor, dass sich das Medianalter bei der Einreise, vor allem für Männer, seit Beginn der 90er Jahre signifikant erhöht hat, was u.a. auf die Verringerung der Zuwanderung von Gastarbeitern aus dem südeuropäischen Raum im Rahmen des Saisonnierstatuts sowie die Zunahme der Zuwanderung von Höherqualifizierten aus westlichen Industriestaaten zurückzuführen ist.

\section{Tab. 8.7: Nationalitätengruppen nach Einwanderungskohorten (in Prozent)}

\begin{tabular}{|c|c|c|c|c|c|c|c|}
\hline \multirow[t]{2}{*}{$\begin{array}{l}\text { Nationalitäten- } \\
\text { gruppen }\end{array}$} & \multicolumn{6}{|c|}{$\begin{array}{c}\text { Einwanderungskohorten } \\
\text { (Aufenthaltsdauer in Jahren) }\end{array}$} & \multirow[t]{2}{*}{$\begin{array}{c}\text { Alter bei } \\
\text { Einreise }\end{array}$} \\
\hline & $<1$ & $1-5$ & $5-10$ & $10-15$ & $15-20$ & $>20$ & \\
\hline \multicolumn{8}{|l|}{ Männer } \\
\hline Nordeuropa & 2.0 & 15.3 & 17.0 & 10.6 & 6.2 & 48.8 & 26 \\
\hline Südeuropa & 1.1 & 2.9 & 11.6 & 17.9 & 10.6 & 56.0 & 22 \\
\hline Nicht-Europa & 1.1 & 10.9 & 29.8 & 31.7 & 10.3 & 16.3 & 24 \\
\hline Total & 1.4 & 8.6 & 16.8 & 18.2 & 9.1 & 46.0 & 24 \\
\hline \multicolumn{8}{|l|}{ Frauen } \\
\hline Nordeuropa & 5.5 & 19.6 & 14.9 & 9.5 & 5.6 & 44.9 & 25 \\
\hline Südeuropa & 1.0 & 9.6 & 16.1 & 12.9 & 10.9 & 49.6 & 23 \\
\hline Nicht-Europa & 0.2 & 23.9 & 22.1 & 23.1 & 10.5 & 20.2 & 23 \\
\hline Total & 2.4 & 15.8 & 16.9 & 13.7 & 9.0 & 42.3 & 24 \\
\hline
\end{tabular}

Quelle: SAKE (1995), eigene Berechnungen

Anmerkungen: Zur Aufschlüsselung der Nationalitätengruppen siehe TAB. 8.2

181 Siehe Anhang 1, TAB. A8.6.

182 Siehe Anhang 1, TAB. A8.6.

183 Siehe Anhang 1, TAB. A8.6 sowie BFA (1998). Aus TAB. A8.6 geht ebenfalls hervor, dass die Verlagerung in den Herkunftsländer bei den Frauen im Vergleich zu Männern erst mit einer gewissen zeitlichen Verzögerung stattgefunden hat. Dieses Ergebnis impliziert, dass ein substantieller Teil der Frauen im Rahmen des Familiennachzugs in die Schweiz eingereist ist. 
Aus TAB. 8.7 lassen sich zwei Erkenntnisse gewinnen. Erstens zeigt die Aufschlüsselung der Nationalitätengruppen nach Einwanderungskohorten, dass mehr als 50 Prozent der Ausländer seit mindestens 15 Jahren in der Schweiz leben, wobei dieses Ergebnis vor allem durch die hohe Aufenthaltsdauer der Einwanderer aus den Anrainerstaaten der Schweiz bedingt ist. Zweitens besteht zwischen den einzelnen Nationalitätengruppen eine relativ grosse Diskrepanz hinsichtlich des Medianalters bei Einreise (siehe auch TAB. A8.7 in Anhang 1). Einwanderer aus hochindustrialisierten Ländern sind bei der Einreise deutlich älter als Zuwanderer aus süd- oder nicht-europäischen Ländern. Dies ist u.a. auf divergierende Qualifikationsprofile und damit verbundene Unterschiede in der Ausbildungszeit zwischen diesen beiden Gruppen zurückzuführen.

\subsection{Empirische Datenbasis: Die Schweizerische Arbeits- KRÄFTEERHEBUNG (SAKE)}

Das System der verschiedenen Statistiken zum Erwerbsleben in der Schweiz wies, trotz seiner zentralen Bedeutung als Bindeglied zwischen der Bevölkerungs- und der Wirtschaftsstatistik, bis Anfang der 90er Jahre erhebliche Lücken auf. ${ }^{184}$ Bis zur Einführung der Schweizerischen Arbeitskräfteerhebung (SAKE) im Jahre 1991 basierten die offiziellen schweizerischen Statistiken zur Erwerbsbevölkerung, Beschäftigung und Arbeitslosigkeit auf einer Kombination von Daten aus unterschiedlichen Quellen, wie der in zehnjährigem Abstand durchgeführten Volkszählung, der vierteljährlichen Beschäftigungsstatistik oder den monatlichen Angaben zu den registrierten Arbeitslosen.

Das Fehlen konsistenter Daten über Beschäftigung und Arbeitslosigkeit aus einer einzigen Quelle, der Mangel an Daten über die sozio-ökonomischen und soziodemographischen Merkmale der Erwerbsbevölkerung zwischen den einzelnen Volkszählungen sowie das Fehlen international vergleichbarer Daten über die Erwerbsbevölkerung, Beschäftigung und Arbeitslosigkeit können als Impulse für die Einführung der jährlich durchgeführten SAKE angesehen werden. ${ }^{185} \mathrm{Im}$ folgenden wird auf die Ziele, Erhebungsmethode sowie Aussagekraft und Grenzen der SAKE eingegangen.

\subsubsection{ZIELE DER SAKE}

Mit der SAKE werden verschiedene Zielsetzungen verfolgt, die sich wie folgt zusammenfassen lassen:

184 Siehe BUHMANN (1992).

185 Siehe BuHMaNN/HussmanNS (1991). 
Tab. 8.8: Zielsetzungen der Schweizerischen Arbeitskräfteerhebung (SAKE)

1. Bereitstellung aktueller und verständlicher Daten über die Grösse der Erwerbsbevölkerung und ihrer Komponenten während der Periode zwischen den Volkszählungen.

2. Bereitstellung aktueller und verständlicher Daten über die Struktur der Erwerbsbevölkerung und ihrer Komponenten nach demographischen Charakteristika, Ausbildungsniveau, Weiterbildung, Wirtschaftszweig, Beruf, beruflicher Stellung, Arbeitszeit, Arbeitsbedingungen und wirtschaftlicher Situation.

3. Messung von Veränderungen über die Zeit in der Grösse und Struktur der Erwerbsbevölkerung und ihrer Komponenten.

4. Aufdeckung der Bestimmungsfaktoren arbeitsmarktrelevanten Verhaltens und Beschreibung der Effekte solchen Verhaltens auf die Lebensbedingungen und die soziale Position der Individuen und Haushalte.

5. Bereitstellung von Daten als Inputfaktoren für andere Statistiken.

6. Bereitstellung sowohl von Querschnittsdaten als auch von Längsschnittsdaten um Flüsse und Veränderungen zwischen und innerhalb der verschiedenen Kategorien der Erwerbsbevölkerung zu ermitteln.

Quelle: Buhmann/Hussmanns (1991)

\subsubsection{DEFINITION VON ERWERBSTÄTIGKEIT UND ERWERBSLOSIGKEIT}

Mit der SAKE erfolgt erstmals eine im Einklang mit den Empfehlungen der Internationalen Arbeitsorganisation (ILO), der OECD und der Europäischen Union stehende Definition von Erwerbstätigkeit und Arbeits- bzw. Erwerbslosigkeit. Mit der SAKE stehen diese Begriffe damit nicht mehr losgelöst nebeneinander, sondern basieren auf einem integrierten Konzept und sind wie folgt definiert:

\section{Tab. 8.9: Definition zentraler Begriffe zur Erwerbstätigkeit in der SAKE}

Erwerbstätig sind nach den internationalen Definitionen:

- Personen, die in der abgeschlossenen Woche vor der Befragung mindestens eine Stunde gegen Entlohnung gearbeitet haben, sei es selbständig oder unselbständig;

- Personen, die in der abgeschlossenen Woche vor der Befragung nicht gearbeitet haben, aber trotzdem eine formelle Arbeitsbeziehung zu einem Arbeitgeber besitzen (Abwesenheit von der Arbeit wegen Krankheit, Ferien, usw.);

- Personen, die als mitarbeitende Familienmitglieder in der abgeschlossenen Woche vor der Befragung im Familienbetrieb gearbeitet haben.

Erwerbslos sind alle Personen in der SAKE, die

- in der abgeschlossenen Woche vor der Befragung nicht erwerbstätig waren und

- in den vier vorangegangenen Wochen eine Arbeit gesucht haben und

- in dieser Zeit eine oder mehrere Suchaktivitäten unternommen haben und

- innerhalb der nächsten vier Wochen mit einer Tätigkeit beginnen könnten.

Nichterwerbstätig sind alle Personen in der SAKE, die in der abgeschlossenen Woche vor der Befragung

- nicht erwerbstätig sind

- nicht erwerbslos sind.

Quelle: Buhmann (1992) 
Aus dieser Neukonzeption in der Erfassung und Messung der verschiedenen Grössen im Bereich Erwerbsleben resultieren definitorische Unterschiede zu den bisherigen Quellen. Diese bestehen hinsichtlich der personellen Abgrenzung, der Mindestarbeitszeit, der Periodizität der Erfassung, der Anwendung des Inlands- oder des Inländerkonzeptes, sowie hinsichtlich der in den verschiedenen Quellen nicht erfassten Personengruppen. Divergenzen in den Ergebnissen können daher einerseits auf die unterschiedliche Auslegung des Begriffs der Erwerbstätigkeit bzw. Erwerbslosigkeit zurückgeführt werden sowie andererseits auf unterschiedliche Messmethoden. Ein Überblick hierzu findet sich in TAB. 8.10.

Die auf der SAKE basierenden Erwerbstätigenzahlen fallen im Vergleich zur traditionellen Erwerbstätigenstatistik deutlich höher aus, da Erwerbstätigkeit in der SAKE umfassender definiert ist und deshalb gewisse Gruppen wie z.B. Minimalerwerbstätige zusätzlich erfasst werden. Die Berücksichtigung aller erwerbstätiger Arbeitsmarktgruppen in der SAKE ermöglicht damit eine vollständige Erfassung des effektiv in der Schweiz geleisteten Arbeitsvolumens. Neben der Statistik der registrierten Arbeitslosen des BWA steht seit der Einführung der SAKE zudem ein zweiter Indikator zur Messung der Arbeitslosigkeit zur Verfügung. ${ }^{186}$ Diese beiden Statistiken zur Arbeitslosigkeit unterscheiden sich im wesentlichen in drei Punkten.

Erstens sind sie durch divergierende Definitionen von Arbeitslosigkeit charakterisiert, was eine Diskrepanz in der Zahl der erfassten Arbeitslosen zur Folge hat. In der Statistik der registrierten Arbeitslosen des BWA werden nur diejenigen Personen erfasst, welche ohne Arbeit sind und die sich bei einem Arbeitsamt zur Arbeitsvermittlung gemeldet haben. In der SAKE werden demgegenüber alle Erwerbslosen erfasst, unabhängig davon, ob sie bei einem Arbeitsamt gemeldet sind oder nicht. Zweitens unterscheiden sich die beiden Statistiken hinsichtlich der Messmethode. Während die Statistik der registrierten Arbeitslosen auf einer Vollerhebung durch die öffentlichen Arbeitsämter basiert, werden die Arbeits- bzw. Erwerbslosen im Rahmen der SAKE über die Befragung einer repräsentativen Stichprobe an Haushalten ermittelt. Drittens geht aus TAB. 8.9 hervor, dass eine Person gemäss SAKE dann als erwerbslos gilt, wenn sie in der Woche vor der Befragung weniger als eine Stunde gegen Entgelt gearbeitet hat. In der BWA-Statistik werden demgegenüber alle Personen erfasst, die in keinem Arbeitsverhältnis stehen, aber auch (und damit in Abweichung zur SAKE-Statistik) jene, die eine Teilzeitbeschäftigung ausüben und eine Vollzeit- oder eine weitere Teilzeitstelle suchen.

186 Siehe CORNIOLEY (1992). 
Tab. 8.10: Statistische Quellen zur Erwerbstätigkeit und Beschäftigung: Regionalisierungsgrad und erfasste Personengruppen

\begin{tabular}{|c|c|c|c|c|c|}
\hline & \multicolumn{5}{|c|}{ Statistische Quellen zur Erwerbstätigkeit und Beschäftigung } \\
\hline & ETS & SAKE & $V Z$ & BZ & BESTA \\
\hline $\begin{array}{l}\text { Regionalisierungs- } \\
\text { grad }\end{array}$ & Schweiz & $\begin{array}{l}\text { Grosse } \\
\text { Regionen }\end{array}$ & $\begin{array}{l}\text { Gemeinden, } \\
\text { Quartiere }\end{array}$ & $\begin{array}{l}\text { Gemeinden, } \\
\text { Quartiere }\end{array}$ & $\begin{array}{l}\text { Kantone, } \\
\text { Grossstädte }\end{array}$ \\
\hline $\begin{array}{l}\text { Erwerbstätige oder } \\
\text { Beschäftigte }\end{array}$ & $\begin{array}{l}\text { Erwerbs- } \\
\text { tätige }\end{array}$ & $\begin{array}{l}\text { Erwerbs- } \\
\text { tätige }\end{array}$ & $\begin{array}{l}\text { Erwerbs- } \\
\text { tätige }\end{array}$ & Beschäftigte & Beschäftigte \\
\hline Mindestarbeitszeit & $\begin{array}{l}6 \text { Std.- } \\
\text { Woche }\end{array}$ & $\begin{array}{l}1 \text { Std.- } \\
\text { Woche }\end{array}$ & $\begin{array}{l}1 \text { Std.- } \\
\text { Woche }\end{array}$ & $\begin{array}{l}6 \text { Std.- } \\
\text { Woche }\end{array}$ & $\begin{array}{l}6 \text { Std.- } \\
\text { Woche }\end{array}$ \\
\hline $\begin{array}{l}\text { Inlands- vs. Inlän- } \\
\text { derkonzept }\end{array}$ & $\begin{array}{l}\text { Inlands- } \\
\text { konzept }\end{array}$ & $\begin{array}{l}\text { Inländer- } \\
\text { konzept }\end{array}$ & $\begin{array}{l}\text { Inländer- } \\
\text { konzept }\end{array}$ & $\begin{array}{l}\text { Inlands- } \\
\text { konzept }\end{array}$ & $\begin{array}{l}\text { Inlands- } \\
\text { konzept }\end{array}$ \\
\hline $\begin{array}{l}\text { Nicht erfasste } \\
\text { Personengruppen }\end{array}$ & & & & & \\
\hline $\begin{array}{l}\text { Botschaftspersonal } \\
\text { im Ausland }\end{array}$ & & nicht erfasst & nicht erfasst & nicht erfasst & nicht erfasst \\
\hline $\begin{array}{l}\text { Mitglieder Schweizer } \\
\text { Hochseeflotte }\end{array}$ & & nicht erfasst & nicht erfasst & nicht erfasst & nicht erfasst \\
\hline Grenzgänger & & nicht erfasst & nicht erfasst & & \\
\hline $\begin{array}{l}\text { Einwohner der } \\
\text { Schweiz, die im } \\
\text { Ausland arbeiten }\end{array}$ & nicht erfasst & & & nicht erfasst & nicht erfasst \\
\hline $\begin{array}{l}\text { Ausl. Diplomaten } \\
\text { mit dipl. Immunität }\end{array}$ & nicht erfasst & & & nicht erfasst & nicht erfasst \\
\hline $\begin{array}{l}\text { Übrig. Personal von } \\
\text { Botschaften, Konsu- } \\
\text { laten \& intern. Org. }\end{array}$ & nicht erfasst & & & nicht erfasst & nicht erfasst \\
\hline Saisonniers & & nicht erfasst & & & \\
\hline Kurzaufenthalter & & nicht erfasst & & & \\
\hline Asylbewerber & & nicht erfasst & & & \\
\hline $\begin{array}{l}\text { Erwerbstätige im } \\
\text { primären Sektor }\end{array}$ & & & & nicht erfasst & nicht erfasst \\
\hline Heimarbeiter & & & & nicht erfasst & nicht erfasst \\
\hline $\begin{array}{l}\text { Angestellte in } \\
\text { Privathaushalten }\end{array}$ & & & & nicht erfasst & nicht erfasst \\
\hline $\begin{array}{l}\text { Selbständige ohne } \\
\text { Betrieb }\end{array}$ & & & & nicht erfasst & nicht erfasst \\
\hline
\end{tabular}

Quelle: BFS (1996a, 1991b).

Anmerkungen: Statistische Quellen: ETS = Erwerbstätigenstatistik, SAKE = Schweizerische Arbeitskräfteerhebung, $V Z=$ Volkszählung, $B Z=$ Betriebszählung, $B E S T A=$ Beschäftigungsstatistik Inländerkonzept: Erfasst wird nur die ständige Wohnbevölkerung (Saisonniers, Grenzgänger, Kurzaufenthalter und Asylbewerber werden nicht berücksichtigt) 


\subsubsection{BEDEUTUNG DER SAKE FÜR DIE WIRTSCHAFTS- UND SOZIALSTATISTIK}

Die SAKE liefert eine umfangreiche Datenquelle für eine Vielzahl unterschiedlicher Fragestellungen im Bereich Erwerbsleben und Arbeitsmarkt. Aus der SAKE lassen sich Informationen über die demographischen Merkmale der Erwerbstätigen, die Arbeitsbedingungen, Berufe, Wirtschaftszweige, Ausbildungsniveaus, Familiensituationen, Einkommen, usw. ermitteln. Ebenso ermöglicht die Registrierung der Erwerbslosenzahlen nicht nur die Erfassung des gesamten Arbeitskräftepotentials sowie damit verbunden des Arbeitsmarktungleichgewichts. Sie erlaubt auch Rückschlüsse auf Ursachen und Wirkungen der Erwerbslosigkeit zu ziehen. Desweiteren wird aufgrund der Informationen über Nichterwerbstätige ein Vergleich mit Erwerbstätigen und Erwerbslosen möglich. Dadurch lassen sich Themen wie der Wiedereinstieg von Frauen in die Erwerbstätigkeit oder die wirtschaftliche Situation von Personen in der Ausbildung oder im Ruhestand erörtern.

Die Befragung eines grossen Teils der Haushalte während mehrerer Jahre ermöglicht zudem eine Beleuchtung der dynamischen Aspekte des Arbeitsmarktes sowie der Arbeitsmarktmobilität im Rahmen von Paneldatenanalysen. So lassen sich z.B. Erkenntnisse über die Häufigkeit von Stellenwechseln oder die Dauer der Erwerbslosigkeit auf Individualebene gewinnen. Schliesslich erlaubt die SAKE aufgrund ihrer Konformität mit internationalen Standards auch internationale Vergleiche bezüglich verschiedener Arbeitsmarktgrössen.

\subsubsection{ERHEBUNGSMETHODE}

Im folgenden wird auf die Periodizität, die Stichprobengrösse sowie die Vorgehensweise der SAKE näher eingegangen. Die SAKE wird in einem jährlichen Rhythmus jeweils im 2. Quartal (April-Juni) durchgeführt. Die jährliche Periodizität der Erhebung ermöglicht die Bereitstellung struktureller Informationen. Um den Erfordernissen bei der Analyse von Konjunkturschwankungen gerecht zu werden, muss jedoch weiterhin auf die vierteljährlichen Daten der Erwerbstätigenstatistik sowie auf die monatlichen Erhebungen der registrierten Arbeitslosen zurückgegriffen werden. Die Wahl des Zeitpunktes der Durchführung im 2. Quartal hat im wesentlichen zwei Ursachen. ${ }^{187}$ Erstens stellt dieser Zeitraum die 'durchschnittlichste' Zeit des Jahres dar. Zweitens wurde er aus Gründen der internationalen Vergleichbarkeit gewählt, da ein Grossteil der Arbeitskräfteerhebungen in anderen Ländern während derselben Periode erfolgt.

Die Grundgesamtheit der Personen auf der die SAKE beruht, umfasst die sog. 'ständige Wohnbevölkerung' der Schweiz, d.h. alle Schweizer Bürger sowie Ausländer mit einer Niederlassungs- oder Jahresaufenthaltsbewilligung. Nicht berücksichtigt werden in der SAKE somit Saisonniers, Kurzaufenthalter, Grenzgänger sowie Asylbewerber. Desweiteren werden nur Personen erfasst, die mindestens 15

187 BUHMANN (1992), BUHMANN/HUSSMANSS (1991). 
Jahre alt sind. Die Beteiligung an der SAKE-Befragung ist für alle Haushalte bzw. Personen freiwillig. Auf der Basis von 24'000 Haushalten konnten so 1991 rund 16 '000 Interviews realisiert werden. ${ }^{188}$ Für das Jahr 1995 erfolgte eine einmalige Verdoppelung der Stichprobengrösse, um eine regionale bzw. kantonale Aufschlüsselung der verschiedenen Messgrössen zu ermöglichen.

Der Stichprobenumfang der SAKE umfasste in der Periode 1991-1994 rund 16'000-18'000 Personen (im Jahre 1995 rund 32'000 Personen), welche über ein vorgegebenes Zufallsverfahren aus dem schweizerischen Telefonregister ausgewählt wurden. Den Referenzpersonen innerhalb eines Haushaltes werden im Rahmen eines telefonischen Interviews Fragen zu verschiedenen Themenkreisen rund um die Erwerbstätigkeit gestellt. Die Ermittlung dieser Referenzperson innerhalb eines Haushaltes erfolgt ebenfalls über ein Zufallsverfahren. Der Anteil der ausländischen Referenzpersonen in der SAKE liegt bei rund 13-14 Prozent und damit rund 5 Prozent unter dem Ausländeranteil an der Gesamtbevölkerung.

Die Ergebnisse der SAKE lassen sich auf die Gesamtbevölkerung, d.h. die ständige Wohnbevölkerung der Schweiz, hochrechnen. Die Gewichtung der Stichprobe berücksichtigt dabei einerseits die durch den Stichprobenplan bedingten divergierenden Auswahlwahrscheinlichkeiten der Haushalte sowie andererseits strukturelle Merkmale wie das Geschlecht, die Altersklasse, oder bei den Ausländern, die Aufenthaltskategorie und die Nationalität. 189 Wie die meisten Arbeitskräfteerhebungen beruht auch die SAKE auf dem Rotationsprinzip. Dies impliziert, dass auf die Gesamtstichprobe eines Jahres rund ein Fünftel Erstbefragungen und vier Fünftel Folgebefragungen entfallen, so dass jedes Jahr 20 Prozent der Personen der Stichprobe ausgetauscht werden. Ein Haushalt bzw. eine Referenzperson kann somit maximal fünf Jahre in der Stichprobe verbleiben. Der daraus resultierende Paneldatensatz ermöglicht so eine Analyse von Veränderungen über die Zeit.

Mit der Revision des Fragebogens im Jahre 1996 fanden einige Modifikationen der SAKE statt, die in erster Linie die Fragen zur beruflichen Stellung sowie zum Arbeitsvolumen der Erwerbstätigen betrafen. Diese Veränderungen haben allerdings zu Problemen hinsichtlich der Vergleichbarkeit zu den Daten der Erhebungen der Jahre 1991 bis 1995 geführt. Aus diesem Grund wurde auf die Verwendung der Daten des Jahres 1996 verzichtet. Im weiteren wurden mit der Revision im Jahre 1996 auch zusätzliche Befragungsmodule zu einem jährlich wechselnden

188 Diese Ergebnisse lassen sich darauf zurückführen, dass im Jahre 1991 rund 4'300 Haushalte auf eine Teilnahme an der Befragung verzichtet haben, 1'400 Adressen ungültig waren und 1'200 Adressen nicht erreicht werden konnten. 1'200 Personen konnten schliesslich nicht befragt werden, weil sie keine der drei Interviewsprachen (Deutsch, Französisch, Italienisch) beherrschten oder weil altersbedingte Probleme ein Interview verunmöglichten.

189 Für das Jahr 1991 repräsentierte eine im Rahmen der SAKE befragte Person rund 350 Personen der ständigen Wohnbevölkerung im Alter von 15 oder mehr Jahren. 
Schwerpunktthema eingeführt. Im Jahre 1996 handelte es sich um das Thema 'Weiterbildung', im Jahre 1997 um das Thema 'unbezahlte Arbeit bzw. ehrenamtliche Tätigkeiten' und im Jahre 1998 stand schliesslich das Thema 'berufliche und räumliche Mobilität' im Zentrum.

\subsubsection{AUSSAGEKRAFT UND GRENZEN DER SAKE}

Die Grenzen der Aussagekraft der SAKE lassen sich vor allem anhand von vier Punkten illustrieren. Erstens bestehen für die ökonometrische Analyse einige Restriktionen, die sich aus der Verfügbarkeit bzw. der Qualität der Daten ergeben. So werden im Rahmen der SAKE lediglich Ausländer mit einer Niederlassungsoder Jahresaufenthaltsbewilligung erfasst. Da Ausländer mit einer Saisonnieroder Grenzgängerbewilligung in der Analyse nicht berücksichtigt werden können, lassen sich lediglich partielle Aussagen hinsichtlich der Arbeitsmarktperformance der ausländischen Erwerbstätigen ableiten. ${ }^{190}$ Zweitens ist aufgrund der Stichprobengrösse nur eine beschränkt disaggregierte Analyse möglich. Die Aggregation kann dementsprechend zu Heterogenitätsproblemen bei der ökonometrischen Analyse führen.

Drittens kann der Erfassungszeitpunkt selbst zu einer Verzerrung der Ergebnisse führen. Dies lässt sich am Beispiel der Jugendarbeitslosigkeit illustrieren. ${ }^{191}$ Diese reagiert nicht nur sensibel auf die Konjunkturlage, sondern lässt auch im Jahresverlauf ein regelmässiges Muster erkennen, da die Arbeitslosigkeit unter Jugendlichen zu Jahresbeginn rückläufig ist und gegen Ende des Jahres wieder zunimmt. Dieser Verlauf ist auf den Ausbildungszyklus zurückzuführen. Es ist daher zu vermuten, dass der Erhebungszeitraum kein repräsentatives Bild der Jugendarbeitslosigkeit zu geben vermag.

Viertens ist die Struktur der Befragungen mit Problemen behaftet. Der schwerwiegendste Nachteil der Telefonbefragung und Stichprobenziehung aus dem Telefonregister der Swisscom besteht darin, dass in der Schweiz rund 5 Prozent der Haushalte über keinen eigenen Telefonanschluss verfügen. Problematisch an dieser Tatsache ist, dass sich diese Haushalte nicht gleichmässig über die Gesamtbevölkerung verteilen. Vielmehr ist der Anteil der Haushalte ohne eigenen Telefonanschluss in gewissen Bevölkerungsgruppen überproportional hoch. Gemäss dem Mikrozensus des Jahres 1984 besitzen vor allem Arbeitslose, Personen in Ausbildung, Einpersonenhaushalte, 18-24-jährige, Ledige, Ausländer und Personen mit

190 In diesem Zusammenhang wäre insbesondere eine detaillierte Analyse der Arbeitsmarktperformance der Ausländer mit einer Saisonnierbewilligung wünschenswert, da in den 80er Jahren gemäss den empirischen Untersuchungen von DHIMA (1991) rund 80 Prozent der Zuwanderung in die Schweiz über das Saisonnierstatut erfolgte. Wie aus Kapitel 4 hervorgeht, hat das Saisonnierstatut jedoch im Verlauf der 90er Jahre an Bedeutung verloren.

Siehe CORNIOLEY (1994). 
einem monatlichen Einkommen von weniger als 3'500 CHF überdurchschnittlich häufig keinen eigenen Telefonanschluss. ${ }^{192}$

Diese Ausführungen zeigen, dass das Vorhandensein eines Telefonanschlusses nicht nur von demographischen Merkmalen beeinflusst wird, sondern auch stark mit der Arbeitsmarktsituation der jeweiligen Wirtschaftssubjekte korreliert ist. Die Schätzungen der SAKE sind daher mit einem gewissen Verzerrungspotential behaftet. Die systematischen Verzerrungen, die einerseits aus der Ziehung der Stichprobe aus dem Telefonregister und andererseits durch die Freiwilligkeit der Teilnahme resultieren, werden jedoch durch eine Nachschichtung der Daten ausgeglichen. Zudem hat eine Studie des Bundesamtes für Statistik gezeigt, dass die Vernachlässigung von Haushalten ohne eigenen Telefonanschluss keinen signifikanten Einfluss auf die Stichprobe besitzt. ${ }^{193}$ Abschliessend ist darauf hinzuweisen, dass die Beschränkung auf drei Interviewsprachen (Deutsch, Französisch und Italienisch) zu Verzerrungen in der Stichprobe führen kann, da zu vermuten ist, dass gerade erwerbslose Ausländer über unzureichende Sprachkenntnisse verfügen.

\subsection{FAZIT}

Die Ausführungen zur Untersuchungsmethode in diesem Kapitel haben die Notwendigkeit einer differenzierten Vorgehensweise bei der empirischen Analyse verdeutlicht. Diese äussert sich einerseits in der Unterscheidung verschiedener Nationalitätengruppen, womit dem Umstand Rechnung getragen werden kann, dass z.T. substantielle Diskrepanzen in den sozio-ökonomischen und sozio-demographischen Merkmalen zwischen Ausländern unterschiedlicher Nationalitäten bestehen. Würde im Rahmen einer aggregierten Analyse nur zwischen In- und Ausländern unterschieden, dann blieben diese Unterschiede fälschlicherweise vernachlässigt.

Andererseits wurde auch ausführlich auf die Bedeutung unterschiedlicher Einwanderungskohorten sowie auf die damit zusammenhängenden Probleme hingewiesen. Es zeigte sich, dass insbesondere die Verwendung von Querschnittsdaten aufgrund der Vermischung von Alters- und Kohorteneffekten zu einer Verzerrung der Ergebnisse führen kann. Diese Probleme lassen sich allerdings durch die Ver-

192 Siehe BFS (1991a).

193 Siehe BFS (1996b). In dieser Studie konnte im wesentlichen gezeigt werden, dass die Einfluss des Stichprobenplans sowie jener von nicht-antwortenden Personen auf die Ergebnisse der SAKE von verhältnismässig geringer Bedeutung ist, dass also die Repräsentativität i.a. gewährleistet ist. Dies gilt insbesondere für die wichtigsten Ausprägungen des Arbeitsmarktstatus (erwerbstätig, erwerbslos). Gewisse stichprobenbedingte Verzerrungen lassen sich demgegenüber bei einigen Branchen oder Berufsgruppen sowie sozio-demographischen Variablen nicht eliminieren. 
wendung von Längsschnittsinformationen im Rahmen von Paneldatenanalysen beheben.

Im zweiten Teil dieses Kapitels wurde auf die empirische Datenbasis sowie die Vor- und Nachteile des Datensets der SAKE eingegangen. Der zentrale Vorteil der SAKE besteht in den umfangreichen Informationen über die sozio-ökonomischen und sozio-demographischen Merkmale der einzelnen Wirtschaftssubjekte. Als gewichtiger Nachteil ist demgegenüber einerseits die Beschränkung des Datensets auf die ständige Wohnbevölkerung der Schweiz, d.h. Schweizer sowie Ausländer mit einer Niederlassungs- oder einer Jahresaufenthaltsbewilligung zu nennen sowie andererseits die geringe Zahl an Beobachtungen in den jeweiligen Stichprobenerhebungen. Diese Nachteile implizieren, dass sich von den Ergebnissen der empirischen Analyse nur in eingeschränktem Masse verallgemeinernde Aussagen über die Performance aller in der Schweiz erwerbstätiger Ausländer ableiten lassen. 


\section{EMPIRISCHE ANALYSE DER BESCHÄFTIGUNGSPERFORMANCE}

Die empirische Analyse der Arbeitsmarktperformance erfolgt über die Beschäftigungs- und Einkommensperformance in diesem sowie im nachfolgenden Kapitel. Um eine umfassende Evaluation zu gewährleisten, werden sowohl produktivitätsbezogene als auch diskriminierungsbedingte Faktoren berücksichtigt. Die deskriptive Analyse in Kapitel 3 hat gezeigt, dass zwischen In- und Ausländern substantielle Unterschiede in der Beschäftigungs- und Erwerbslosigkeitsstruktur bestehen. Diese Zusammenhänge werden im Rahmen der empirischen Untersuchungen zur Beschäftigungsperformance unter Verwendung ökonometrischer Verfahren sowie deskriptiver Statistiken eingehend erörtert. Die Zielsetzung dieses Kapitels besteht einerseits darin zu erörtern, ob zwischen In- und Ausländern signifikante Unterschiede in den Beschäftigungs- und Erwerbslosigkeitsprofilen bestehen. Andererseits gilt es zu untersuchen, worauf sich diese Unterschiede zurückführen lassen.

Die Vorgehensweise gestaltet sich dabei wie folgt. In einem ersten Schritt werden zur Motivation der Analyse einige deskriptive Statistiken präsentiert. In einem zweiten Schritt werden produktivitätsbezogene Determinanten der Beschäftigungsperformance, im Rahmen von Probit-Schätzungen der Erwerbslosigkeitswahrscheinlichkeit, analysiert. In einem dritten Schritt werden diskriminierungsbedingte Einflussfaktoren über die Untersuchung der Arbeitsmarktsegmentation erörtert. In einem vierten Schritt folgt schliesslich ein kurzes Fazit.

\subsection{DESKRIPTIVE ANALYSE DER BESCHÄFTIGUNGSPERFORMANCE}

Die deskriptive Analyse dieses ersten Abschnitts vermag bereits erste Hinweise auf Performanceunterschiede zwischen In- und Ausländern sowie zwischen den verschiedenen Nationalitätengruppen zu geben. Die Analyse dieses Abschnitts basiert, analog zur Analyse der Arbeitsmarktsegmentation in Abschnitt 9.3, auf der auf die ständige Wohnbevölkerung hochgerechneten Stichprobe der SAKE des Jahres 1995.194

Die Analyse dieses Abschnitts erfolgt in drei Schritten. In einem ersten Schritt wird auf den Humankapitalbestand der einzelnen Wirtschaftssubjekte eingegangen. Die Humankapitalausstattung wird dabei über die höchste abgeschlossene Ausbildung approximiert. Diese Variable stellt eine wichtige Determinante sowohl

194 Die ökonometrische Analyse basiert demgegenüber, mit Ausnahme der Untersuchungen zur Arbeitsmarktsegmentation, auf den Daten der ungewichteten, d.h. der nicht auf die Gesamtbevölkerung hochgerechneten Stichprobe. 
der Beschäftigungs- als auch der Einkommensperformance dar. In einem zweiten Schritt folgt die Analyse der beruflichen Stellung sowie der Beschäftigung der Erwerbstätigen nach Wirtschaftszweigen. Diese Analyse gibt erste Hinweise auf die hierarchische und branchenspezifische Segregation der in- und ausländischen Wirtschaftssubjekte. Schliesslich erfolgt in einem dritten Schritt mit der Analyse der Erwerbsbeteiligung bzw. Erwerbslosigkeit die direkte Analyse der Beschäftigungsperformance. Um aussagekräftige Ergebnisse zu gewährleisten, findet gemäss den in Kapitel 7 formulierten Hypothesen, eine Differenzierung der Analyse nach geschlechts-, nationalitäten- und kohortenspezifischen Merkmalen statt.

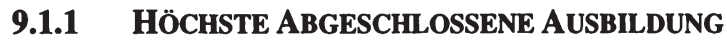

Das Qualifikationsprofil stellt eines der wichtigsten, wenn nicht das wichtigste Merkmal für eine korrekte Einschätzung der Arbeitsmarktperformance dar. Ein Problem bei der Beurteilung der Qualifikationen von Ausländern ergibt sich allerdings aus der unvollständigen Vergleichbarkeit der im Ausland erworbenen Qualifikationen, d.h. schulischer Abschlüsse und beruflicher Ausbildung, mit schweizerischen Abschlüssen. Ein weiteres Problem bei der Beurteilung der Qualifikationen der Ausländer besteht darin, dass im Rahmen der SAKE keine Informationen darüber zur Verfügung stehen, ob die Qualifikationen im In- oder Ausland erworben wurden. Wie die theoretische Analyse jedoch gezeigt hat, wäre mit Blick auf die Transferierbarkeit sowie die Akkumulation von ziellandspezifischem $\mathrm{Hu}-$ mankapital eine solche Unterscheidung von Bedeutung.

In TAB. 9.1 und 9.2 ist, nach Geschlecht getrennt, das durchschnittlich erreichte Ausbildungsniveau für die verschiedenen Nationalitätengruppen und Einwanderungskohorten abgebildet. Die wichtigsten Ergebnisse aus TAB. 9.1 lassen sich wie folgt zusammenfassen. Erstens ist sowohl für Männer als auch für Frauen deutlich erkennbar, dass signifikante nationalitätenspezifische Unterschiede im Ausbildungsniveau bestehen. Es zeigt sich, dass mehr als 55 bzw. 75 Prozent der Männer und Frauen aus Südeuropa sowie rund 32 bzw. 47 Prozent der Einwanderer aus Nicht-Europa lediglich eine obligatorische Grundschulausbildung oder keinen Abschluss bzw. eine andere Ausbildung besitzen. Es ist in diesem Zusammenhang davon auszugehen, dass ein Grossteil dieser Erwerbstätigen im Rahmen des Saisonnierstatuts eingewandert ist, dem bei der Zuwanderung gering qualifizierter Arbeitskräfte eine Art Schleusenfunktion zukommt. ${ }^{195}$

Zweitens besteht für die männlichen Einwanderer aus nicht-europäischen Staaten eine augenfällige Heterogenität der Qualifikationsstruktur, mit rund einem Drittel gering Qualifizierter und einem Fünftel hoch Qualifizierter. Mit Blick auf TAB. A9.1 in Anhang 1 ist ersichtlich, dass dieses Ergebnis im wesentlichen durch Einwanderer aus aussereuropäischen Staaten bedingt ist. Diese Nationalitäten-

195 Siehe DHIMA (1991). 
gruppe ist durch ein hohes Aggregationsniveau gekennzeichnet (siehe TAB. 8.2). Bei der Bewertung der Ergebnisse der empirischen Analyse gilt es diesen Umstand zu berücksichtigen.

Drittens ist auf die vergleichsweise besseren Ausbildungsprofile der Zuwanderer aus nordeuropäischen Staaten hinzuweisen. Dieses Ergebnis ist einerseits darauf zurückzuführen, dass das durchschnittliche Qualifikationsniveau in diesen Staaten über jenem in den süd- und nicht-europäischen Länder liegt. Andererseits ist davon auszugehen, dass zwischen diesen Regionen Unterschiede in der Selektion bestehen, welche die Dualität der Rekrutierung bzw. Beschäftigung ausländischer Arbeitskräfte reflektiert. Es ist davon auszugehen, dass aus süd- und nicht-europäischen Staaten vorwiegend gering qualifizierte Arbeitskräfte für schlecht bezahlte, einfache und manuelle Tätigkeiten rekrutiert werden. Aufgrund des grossen internationalen Lohngefälles sind diese Arbeitsplätze aber dennoch attraktiv. Aus dem nordeuropäischen Raum ist demgegenüber aufgrund der unterschiedlichen Anreizstrukturen vor allem mit der Rekrutierung höher qualifizierter Arbeitskräfte zu rechnen.

Tab. 9.1: Höchste abgeschlossene Ausbildung der erwerbstätigen Bevölkerung nach Nationalitätengruppen (in Prozent)

\begin{tabular}{|c|c|c|c|c|c|c|}
\hline \multirow{2}{*}{$\begin{array}{l}\text { Nationalitäten- } \\
\text { gruppen. }\end{array}$} & \multicolumn{6}{|c|}{ Höchste Abgeschlossene Ausbildung } \\
\hline & 1 & 2 & 3 & 4 & 5 & 6 \\
\hline \multicolumn{7}{|l|}{ Männer } \\
\hline Nordeuropa & 4.8 & 25.7 & 14.6 & 18.5 & 30.9 & 5.5 \\
\hline Südeuropa & 42.7 & 27.7 & 9.4 & 4.2 & 2.5 & 13.5 \\
\hline Nicht-Europa & 14.8 & 29.0 & 10.7 & 8.5 & 20.1 & 16.9 \\
\hline Ausländer & 24.7 & 27.3 & 11.3 & 9.8 & 15.3 & 11.6 \\
\hline Schweizer & 7.4 & 46.4 & 13.0 & 20.1 & 9.5 & 3.7 \\
\hline \multicolumn{7}{|l|}{ Frauen } \\
\hline Nordeuropa & 7.4 & 26.5 & 26.7 & 8.2 & 19.6 & 11.5 \\
\hline Südeuropa & 54.0 & 15.0 & 9.0 & 1.1 & 1.7 & 19.2 \\
\hline Nicht-Europa & 30.7 & 28.5 & 14.3 & 5.3 & 4.9 & 16.4 \\
\hline Ausländer & 34.3 & 21.2 & 16.2 & 4.3 & 8.4 & 15.7 \\
\hline Schweizer & 18.2 & 42.1 & 24.1 & 5.3 & 5.0 & 5.3 \\
\hline
\end{tabular}

Quelle: SAKE (1995), eigene Berechnungen

Anmerkungen: Enwerbstätige Bevölkerung: Personen zwischen 16 und 64 Jahren

Zur Aufschlüsselung der Nationalitätengruppen siehe TAB. 8.2

Höchste abgeschlossene Ausbildung: 1 = Obligatorische Grundschule, $2=$ Berufslehre, 3 = Vollzeitberufsschule, Diplommittelschule, Matura, 4 = Höhere Berufsausbildung, $5=$ Universität/Hochschule, $6=$ Kein Abschluss, andere Ausbildung

Aus TAB. 9.1 ist desweiteren ersichtlich, dass neben diesen nationalitätenspezifischen Unterschieden auch substantielle geschlechtsspezifische Unterschiede in 
den Ausbildungsniveaus bestehen. Der Vergleich zeigt, dass der Anteil der Frauen in den höchsten Ausbildungssegmenten bei allen Nationalitätengruppen z.T. deutlich unter jenem der Männer liegt. OAXACA (1973) und RoSEN (1977) haben in diesem Zusammenhang bereits in den 70er Jahren darauf hingewiesen, dass geschlechtsspezifische Unterschiede in den Erwerbstätigkeitsprofilen über den Lebenszyklus dazu führen, dass für Männer und Frauen divergierende Anreize zu Investitionen in Humankapital bestehen. Im speziellen lässt die durchschnittlich kürzere Erwerbstätigkeitsphase der Frauen auf geringere Investitionsanreize schliessen.

Von Interesse ist schliesslich auch der Vergleich der Qualifikationen von In- und Ausländern. Zwei Punkte gilt es in diesem Zusammenhang hervorzuheben. Erstens verdeutlichen nationalitätenspezifische Unterschiede in den Qualifikationen die Notwendigkeit einer disaggregierten Analyse. Zweitens sind Ausländer sowohl in den tiefsten als auch in den höchsten Ausbildungssegmenten stärker vertreten als Inländer. Dieses Ergebnis ist auf den überdurchschnittlich hohen Anteil an gering (hoch) qualifizierten Arbeitskräften aus süd- und nicht-europäischen (nordeuropäischen und aussereuropäischen) Staaten zurückzuführen und unterstreicht damit die vorangegangenen Überlegungen zur Dualität der Rekrutierung ausländischer Arbeitskräfte.

Neben der Bedeutung geographisch-kultureller Faktoren gilt es schliesslich auch dem Einfluss kohortenspezifischer Unterschiede zu berücksichtigen. Es geht dabei um die Frage, ob und wie sich die 'Qualität' der Einwanderer im Zeitverlauf verändert hat. Aus TAB. 9.2 geht hervor, dass sowohl bei den Männern als auch bei den Frauen eine Verbesserung der Humankapitalausstattung über die Zeit stattgefunden hat.

Mit Blick auf die männlichen Erwerbstätigen ist erkennbar, dass sich der Anteil der gering Qualifizierten von der ältesten zur jüngsten Einwanderungskohorte von rund 60 Prozent auf 20 Prozent verringert hat. Gleichzeitig ist der Anteil der hoch Qualifizierten von rund 20 Prozent auf über 60 Prozent angestiegen. Bei den weiblichen Erwerbstätigen hat zwar auch eine Verringerung des Anteils der gering Qualifizierten stattgefunden. Diese Verlagerung hat jedoch vorwiegend zugunsten der intermediären Ausbildungsstufen stattgefunden. Mit Ausnahme der jüngsten Einwanderungskohorte ist ebenfalls eine starke Zunahme des Anteils der Einwanderer ohne Abschluss bzw. mit einer anderen Ausbildung zu verzeichnen. Eine Erklärung für diese Entwicklung ist in der Verlagerung der Herkunftsländer in den nicht-europäischen Raum während der vergangenen Jahre zu sehen. ${ }^{196}$

196 Siehe auch TAB. 8.6 und 8.7. 
Tab. 9.2: Höchste abgeschlossene Ausbildung der erwerbstätigen Bevölkerung nach Einwanderungskohorten (in Prozent)

\begin{tabular}{|c|c|c|c|c|c|c|}
\hline \multirow{2}{*}{$\begin{array}{l}\text { Einwande- } \\
\text { rungskohorten }\end{array}$} & \multicolumn{6}{|c|}{ Höchste Abgeschlossene Ausbildung } \\
\hline & 1 & 2 & 3 & 4 & 5 & 6 \\
\hline \multicolumn{7}{|l|}{ Männer } \\
\hline$<1$ & & 18.7 & 13.1 & 9.6 & 53.1 & 5.5 \\
\hline $1-5$ & 5.5 & 12.6 & 12.0 & 9.8 & 41.6 & 18.4 \\
\hline $5-10$ & 20.7 & 25.1 & 17.6 & 8.0 & 19.6 & 9.0 \\
\hline $10-15$ & 27.0 & 22.3 & 13.5 & 11.0 & 12.7 & 13.5 \\
\hline $15-20$ & 33.8 & 25.0 & 6.6 & 8.4 & 13.9 & 12.3 \\
\hline$>20$ & 28.0 & 34.1 & 8.7 & 10.3 & 8.4 & 10.5 \\
\hline \multicolumn{7}{|l|}{ Frauen } \\
\hline$<1$ & 14.6 & 10.4 & 38.8 & 3.6 & 18.7 & 14.0 \\
\hline $1-5$ & 10.2 & 27.3 & 17.5 & 4.7 & 18.8 & 21.5 \\
\hline $5-10$ & 40.3 & 16.0 & 17.6 & 7.8 & 5.7 & 12.6 \\
\hline $10-15$ & 46.3 & 14.6 & 21.5 & 3.0 & 4.2 & 10.3 \\
\hline $15-20$ & 26.8 & 21.6 & 14.8 & 4.5 & 7.6 & 24.6 \\
\hline$>20$ & 38.6 & 24.8 & 12.0 & 3.1 & 6.8 & 14.7 \\
\hline
\end{tabular}

Quelle: SAKE (1995), eigene Berechnungen

Anmerkungen: Erwerbstätige Bevölkerung: Personen zwischen 16 und 64 Jahren

Einwanderungskohorten: Aufenthaltsdauer in Jahren

Höchste abgeschlossene Ausbildung: 1 = Obligatorische Grundschule, $2=$ Berufslehre, 3

= Vollzeitberufsschule, Diplommittelschule, Matura, 4 = Höhere Berufsausbildung, 5 =

Universität/Hochschule, $6=$ Kein Abschluss, andere Ausbildung

Die Analyse der Qualifikationen in- und ausländischer Erwerbstätiger hat gezeigt, dass zwar eine Verbesserung der 'Qualität' der Zuwanderer stattgefunden hat, die vor allem auf die Verringerung des Anteils der Gastarbeiter sowie die Zunahme des Anteils der Ausländer aus Nordeuropa zurückzuführen ist. Gleichzeitig hat aber die Verlagerung der Zuwanderung in den nicht-europäischen Raum zu einer gegenläufigen Entwicklung geführt, die sich in einem markanten Anstieg der Ausländer ohne Abschluss bzw. mit einem anderen Abschluss widerspiegelt.

\subsubsection{BERUfLiche STELLUNG}

In diesem sowie im nächsten Unterabschnitt wird auf die berufliche Stellung der In- und Ausländer sowie die Beschäftigung nach Wirtschaftszweigen eingegangen. Diese Analyse liefert nicht nur wichtige Hinweise auf die Beschäftigungsperformance, sondern auch auf die Segmentation des Arbeitsmarktes. Wie bereits in Kapitel 6 ausgeführt wurde, lassen sich grundsätzlich zwei Formen der Arbeitsmarktsegmentation unterscheiden: hierarchische und branchenspezifische. Im ersten Fall liegt eine vertikale Aufspaltung des Arbeitsmarktes in Segmente entlang 
verschiedener Hierarchiestufen vor. Im zweiten Fall besteht eine horizontale Segmentation entlang verschiedener Branchen bzw. Wirtschaftszweige.

Während in diesem Abschnitt die Analyse der hierarchischen Segregation im Vordergrund steht, wird im Rahmen des nächsten Abschnitts die branchenspezifische Segregation erörtert. Zur Untersuchung der hierarchischen Segregation wird auf die im Rahmen der SAKE erhobenen Daten über die berufliche Stellung der befragten Personen zurückgegriffen. Aufgrund des tiefen Differenzierungsgrades bei der Definition der beruflichen Stellung wird die hierarchische Segregation lediglich deskriptiv analysiert. Es ist an dieser Stelle allerdings darauf hinzuweisen, dass die Ausübung einer selbständigen Erwerbstätigkeit für Ausländer bewilligungspflichtig ist und die Vergabe dieser Bewilligungen i.d.R. restriktiv gehandhabt wird. Lediglich Ausländer mit einer Niederlassungsbewilligung haben ein Recht auf Ausübung einer selbständigen Erwerbstätigkeit. ${ }^{197}$

Tab. 9.3: Berufliche Stellung der erwerbstätigen Bevölkerung nach Nationalitätengruppen (in Prozent)

\begin{tabular}{|c|c|c|c|c|c|c|}
\hline \multirow{2}{*}{$\begin{array}{l}\text { Nationalitäten- } \\
\text { gruppen }\end{array}$} & \multicolumn{6}{|c|}{ Berufliche Stellung } \\
\hline & 1 & 2 & 3 & 4 & 5 & 6 \\
\hline \multicolumn{7}{|l|}{ Männer } \\
\hline Nordeuropa & 17.7 & 16.9 & 28.0 & 36.7 & & 0.7 \\
\hline Südeuropa & 9.2 & 4.2 & 17.3 & 68.5 & 0.2 & 0.5 \\
\hline Nicht-Europa & 8.5 & 3.2 & 14.3 & 72.2 & 0.5 & 1.3 \\
\hline Ausländer & 12.0 & 8.2 & 20.2 & 58.6 & 0.3 & 0.7 \\
\hline Schweizer & 20.7 & 10.6 & 23.1 & 39.8 & 5.0 & 0.8 \\
\hline \multicolumn{7}{|l|}{ Frauen } \\
\hline Nordeuropa & 12.0 & 4.4 & 17.1 & 64.8 & & 1.7 \\
\hline Süideuropa & 4.4 & 1.7 & 3.6 & 89.8 & 0.2 & 0.3 \\
\hline Nicht-Europa & 4.3 & 1.7 & 8.0 & 84.1 & 1.9 & \\
\hline Ausländer & 8.0 & 2.6 & 9.0 & 79.3 & 0.4 & 0.7 \\
\hline Schweizer & 17.1 & 3.6 & 11.8 & 61.4 & 4.6 & 1.4 \\
\hline
\end{tabular}

Quelle: SAKE (1995), eigene Berechnungen

Anmerkungen: Enwerbstätige Bevölkerung: Personen zwischen 16 und 64 Jahren

Zur Aufschlüsselung der Nationalitätengruppen siehe TAB. 8.2

Berufliche Stellung: 1 = Selbständige und mitarbeitende Familienangehörige, 2 = Arbeitnehmer in Unternehmensleitung, $3=$ Arbeitnehmer mit Vorgesetztenfunktion, $4=$ Arbeitneh mer ohne Vorgesetztenfunktion, 5 = Lehrlinge, 6 = Andere Erwerbstätige (keine Zuordnung möglich)

Aus TAB. 9.3 geht hervor, dass zwischen dem Qualifikationsprofil und der beruflichen Stellung grosse Parallelen bestehen. Dies lässt vermuten, dass die Ausbil-

197 Siehe Kapitel 2. 
dung eine zentrale Determinante der Beschäftigungs- und Einkommensperformance ist. Analog zur Ausbildung lassen sich geschlechts- und nationalitätenspezifische Unterschiede beobachten. Es ist erkennbar, dass sowohl bei den Männern als auch bei den Frauen mehr als zwei Drittel der Einwanderer aus dem süd- und nicht-europäischen Raum als Arbeitnehmer ohne Vorgesetztenfunktion beschäftigt sind. Die Heterogenität hinsichtlich der beruflichen Stellung für Einwanderer aus dem nicht-europäischen Raum ist analog zur Ausbildung durch die Zuwanderer aus aussereuropäischen Staaten bedingt (siehe TAB. A9.3 in Anhang 1). Im Vergleich dazu sind rund 60 Prozent der männlichen Einwanderer aus Nordeuropa in den oberen Segmenten zu finden (der Anteil bei den Frauen liegt zwischen 15 und 35 Prozent).

Tab. 9.4: Berufliche Stellung der erwerbstätigen Bevölkerung nach Einwanderungskohorten (in Prozent)

\begin{tabular}{|c|c|c|c|c|c|c|}
\hline \multirow{2}{*}{$\begin{array}{l}\text { Einwande- } \\
\text { rungskohorten }\end{array}$} & \multicolumn{6}{|c|}{ Berufliche Stellung } \\
\hline & 1 & 2 & 3 & 4 & 5 & 6 \\
\hline \multicolumn{7}{|l|}{ Männer } \\
\hline$<1$ & 14.5 & 23.7 & 10.4 & 43.8 & 5.5 & 2.0 \\
\hline $1-5$ & 6.8 & 11.8 & 17.3 & 59.0 & 2.2 & 2.8 \\
\hline $5-10$ & 7.6 & 6.0 & 22.8 & 62.9 & 0.2 & 0.6 \\
\hline $10-15$ & 8.7 & 6.3 & 17.7 & 66.2 & & 1.1 \\
\hline $15-20$ & 19.1 & 3.2 & 24.8 & 52.9 & & \\
\hline$>20$ & 14.6 & 9.9 & 20.3 & 55.1 & & 0.3 \\
\hline \multicolumn{7}{|l|}{ Frauen } \\
\hline$<1$ & 4.1 & & & 95.9 & & \\
\hline $1-5$ & 3.2 & 3.6 & 8.0 & 81.7 & & 3.5 \\
\hline $5-10$ & 4.6 & 2.5 & 11.1 & 78.6 & 2.5 & 0.7 \\
\hline $10-15$ & 6.7 & 2.1 & 3.3 & 87.9 & & \\
\hline $15-20$ & 6.6 & 3.7 & 13.2 & 76.6 & & \\
\hline$>20$ & 10.2 & 2.4 & 10.3 & 77.1 & & \\
\hline
\end{tabular}

Quelle: SAKE (1995), eigene Berechnungen

Anmerkungen: Erwerbstätige Bevölkerung: Personen zwischen 16 und 64 Jahren

Einwanderungskohorten: Aufenthaltsdauer in Jahren

Berufliche Stellung: $I$ = Selbständige und mitarbeitende Familienangehörige, $2=$ Arbeitnehmer in Unternehmensleitung, $3=$ Arbeitnehmer mit Vorgesetztenfunktion, $4=$ Arbeitnehmer ohne Vorgesetztenfunktion, 5 = Lehrlinge, 6 = Andere Erwerbstätige (keine Zuordnung möglich)

Neben den nationalitätenspezifischen Unterschieden bestehen auch beträchtliche geschlechtsspezifische Unterschiede in der beruflichen Stellung. Die tieferen Ausbildungsniveaus spiegeln sich in der stärkeren Konzentration der Beschäftigung der Frauen in den unteren Hierarchiestufen wider. Der Vergleich zwischen 
In- und Ausländern zeigt auch, dass Schweizer im Mittel eine höhere berufliche Stellung erreichen als Ausländer. Diese Aussage gilt jedoch nur für Einwanderer aus dem süd- und nicht-europäischen Raum. Männliche Zuwanderer aus dem nordeuropäischen Raum, insbesondere aus Deutschland und den sonstigen EU/EFTA-Staaten, sind demgegenüber in höheren beruflichen Stellungen stärker vertreten als Schweizer. Im Vergleich dazu fallen die Unterschiede zwischen Schweizerinnen und Nordeuropäerinnen relativ gering aus.

In TAB. 9.4 sind bei der Analyse der beruflichen Stellung nach Einwanderungskohorten vor allem die geschlechtsspezifischen Unterschiede augenfällig. Bei den Frauen liegt der Anteil der Erwerbstätigen ohne Vorgesetztenfunktion durchgehend bei mindestens 75 Prozent. Bei den Männern fällt demgegenüber auf, dass der Anteil der Beschäftigten mit Funktionen in der Unternehmensleitung von den jüngeren zu den älteren Einwanderungskohorten deutlich abnimmt. Dieses Ergebnis lässt sich mit der verstärkten Zuwanderung aus dem nordeuropäischen Raum in den vergangenen Jahren erklären.

\subsubsection{WiRTSCHAFTSZWEIGE}

Um eine umfassende Analyse der Beschäftigungsperformance zu gewährleisten, werden in diesem Abschnitt die Beschäftigungsanteile der verschiedenen Nationalitätengruppen und Einwanderungskohorten an den unterschiedlichen Wirtschaftszweigen diskutiert. Diese Analyse liefert gleichzeitig Anhaltspunkte für die Untersuchung der zweiten Dimension der Arbeitsmarktsegmentation - die branchenspezifische Segregation (siehe Abschnitt 9.3). Während in diesem Abschnitt lediglich 9 Wirtschaftszweige unterschieden werden (Ein-Steller Niveau), stellt die Analyse der Arbeitsmarktsegmentation bzw. Berechnung der SegmentationsIndizes auf eine detailliertere Definition der Wirtschaftszweige ab (Zwei-Steller Niveau), die eine Unterscheidung von insgesamt 66 Branchen erlaubt.

In $\mathrm{TAB} .9 .5$ sind wiederum eine Reihe nationalitäten- und geschlechtsspezifischer Unterschiede erkennbar. Erstens sind rund drei Viertel der männlichen Einwanderer aus Südeuropa in der Verarbeitenden Produktion, dem Baugewerbe oder dem Handel bzw. Gastgewerbe tätig. Dies gilt im wesentlichen auch für die Einwanderer aus Nicht-Europa, wobei allerdings rund ein Viertel der Einwanderer aus aussereuropäischen Staaten im Banken-/Versicherungs- und Immobiliensektor sowie in den sonstigen Dienstleistungssegmenten beschäftigt ist (siehe TAB. A9.5 in Anhang 1). Diese Ergebnisse decken sich mit den Überlegungen zur Dualität der Arbeitsmarktstruktur bzw. Rekrutierung.

Für Einwanderer aus Nordeuropa fällt das Ergebnis weniger eindeutig aus. Einerseits bestehen relativ hohe Beschäftigungsanteile in der Verarbeitenden Produktion sowie im Handel und Gastgewerbe. Dies gilt aber andererseits auch für den Banken-/Versicherungs- und Immobiliensektor. Diese Dualität in der Beschäftigung ist darauf zurückzuführen, dass sowohl bei den jüngsten, im Mittel höher 
qualifizierten Einwanderungskohorten, als auch bei den ältesten, im Mittel geringer qualifizierten, der Anteil der Nordeuropäer relativ hoch ist (siehe TAB. 8.6 und 8.7). Während diese mehrheitlich in der Verarbeitenden Produktion sowie im Handel und Gastgewerbe tätig sind, sind jene überwiegend in gehobenen beruflichen Positionen im Banken-/Versicherungs- und Immobiliensektor beschäftigt.

Tab. 9.5: Erwerbstätige Bevölkerung nach Wirtschaftszweigen und Nationalitätengruppen (in Prozent)

\begin{tabular}{|c|c|c|c|c|c|c|c|c|c|}
\hline \multirow{2}{*}{$\begin{array}{l}\text { Nationalitä- } \\
\text { tengruppen }\end{array}$} & \multicolumn{9}{|c|}{ Wirtschaftszweige } \\
\hline & 1 & 2 & 3 & 4 & 5 & 6 & 7 & 8 & 9 \\
\hline \multicolumn{10}{|l|}{ Männer } \\
\hline Nordeuropa & 0.5 & & 27.3 & 6.7 & 19.4 & 1.0 & 17.4 & 25.3 & 2.3 \\
\hline Südeuropa & 0.9 & 0.3 & 35.7 & 27.2 & 16.9 & 3.1 & 8.0 & 6.9 & 1.0 \\
\hline Nicht-Europa & 0.2 & & 35.7 & 11.8 & 20.8 & 7.3 & 9.4 & 12.3 & 2.5 \\
\hline Ausländer & 0.7 & 0.2 & 32.9 & 17.5 & 18.4 & 3.2 & 11.4 & 14.0 & 1.7 \\
\hline Schweizer & 6.1 & 1.4 & 22.3 & 9.3 & 16.1 & 8.5 & 16.0 & 13.9 & 6.4 \\
\hline \multicolumn{10}{|l|}{ Frauen } \\
\hline Nordeuropa & & & 10.4 & 0.2 & 19.0 & 4.6 & 9.8 & 49.0 & 7.1 \\
\hline Südeuropa & 0.2 & & 26.2 & & 29.4 & 3.5 & 12.5 & 27.4 & 0.9 \\
\hline Nicht-Europa & & & 30.1 & & 24.0 & 3.7 & 10.0 & 30.5 & 1.7 \\
\hline Ausländer & 0.9 & & 21.4 & 0.1 & 24.6 & 3.8 & 11.1 & 35.0 & 3.1 \\
\hline Schweizer & 4.4 & 0.3 & 11.5 & 2.6 & 24.7 & 4.1 & 15.4 & 32.9 & 4.1 \\
\hline
\end{tabular}

Quelle: SAKE (1995), eigene Berechnungen

Anmerkungen: Erwerbstätige Bevölkerung: Personen zwischen 16 und 64 Jahren

Zur Aufschlüsselung der Nationalitätengruppen siehe TAB. 8.2

Wirtschaftszweige: 1 = Land-, Forstwirtschaft, 2 = Energie-/Wasserversorgung, Bergbau, 3 = Verarbeitende Produktion, $4=$ Baugewerbe, $5=$ HandeVGastgewerbe, $6=$ Ver . kehr/Nachrichten, $7=$ Banken/Versicherungen/Immobilien, $8=$ Sonstige Dienstleistungen (Gesundheitswesen, Erziehung, Forschung), 9 = Öffentliche Verwaltung

Zweitens zeigt sich mit Blick auf die geschlechtsspezifischen Unterschiede, dass der Anteil der Frauen bei den sonstigen Dienstleistungen durchgehend über jenem der Männer liegt. Aufgrund des Aggregationsniveaus innerhalb dieses Wirtschaftszweiges lassen sich jedoch keine Aussagen über spezifische Beschäftigungsanteile machen (siehe TAB. A9.17). Drittens geht aus TAB. 9.5 hervor, dass zwischen in- und ausländischen Erwerbstätigen deutliche Unterschiede in den sektoralen Beschäftigungsanteilen bestehen. Im primären Sektor ist die Beschäftigung weitgehend von inländischen Arbeitnehmern dominiert. Dies ist u.a. auf die gesetzlichen Bestimmungen zur Erwerbstätigkeit ausländischer Arbeitskräfte zu- 
rückzuführen. ${ }^{198}$ Im sekundären Sektor ist der Ausländeranteil demgegenüber deutlich höher. Im tertiären Sektor liegt der Beschäftigungsanteil der Inländer wiederum über jenem der Ausländer. Die detaillierte Darstellung in Tab. A9.5 zeigt jedoch, dass die Anteile im höherqualifizierten Dienstleistungssegment, insbesondere bei den männlichen Einwanderern aus Nordeuropa, über jenen der Inländer liegen.

Tab. 9.6: Erwerbstätige Bevölkerung nach Wirtschaftszweigen und Einwanderungskohorten (in Prozent)

\begin{tabular}{|c|c|c|c|c|c|c|c|c|c|}
\hline \multirow{2}{*}{$\begin{array}{l}\text { Einwande- } \\
\text { rungskohorten }\end{array}$} & \multicolumn{9}{|c|}{ Wirtschaftsweige } \\
\hline & 1 & 2 & 3 & 4 & 5 & 6 & 7 & 8 & 9 \\
\hline \multicolumn{10}{|l|}{ Männer } \\
\hline$<1$ & & & 16.8 & 11.7 & 27.9 & & & 32.7 & 10.9 \\
\hline $1-5$ & & & 23.1 & 4.4 & 13.8 & 4.0 & 21.6 & 31.7 & 1.5 \\
\hline $5-10$ & 0.4 & & 29.1 & 18.8 & 14.6 & 4.9 & 13.9 & 18.1 & 0.3 \\
\hline $10-15$ & 0.2 & & 30.8 & 19.3 & 17.8 & 4.1 & 13.4 & 13.3 & 1.2 \\
\hline $15-20$ & 1.7 & & 19.4 & 33.7 & 21.2 & 2.4 & 8.1 & 11.2 & 2.3 \\
\hline$>20$ & 0.9 & 0.4 & 40.7 & 15.5 & 20.2 & 2.3 & 8.6 & 9.1 & 2.2 \\
\hline \multicolumn{10}{|l|}{ Frauen } \\
\hline$<1$ & & & 2.1 & & 27.9 & & 12.5 & 42.9 & 14.5 \\
\hline $1-5$ & & & 13.5 & & 19.0 & 5.9 & 10.8 & 45.8 & 4.9 \\
\hline $5-10$ & & & 20.6 & & 27.2 & 0.3 & 9.5 & 38.6 & 2.9 \\
\hline $10-15$ & & & 23.0 & & 24.1 & 1.7 & 8.5 & 40.7 & 2.1 \\
\hline $15-20$ & & & 22.0 & & 28.4 & 8.1 & 10.6 & 26.3 & 4.6 \\
\hline$>20$ & 0.3 & & 25.8 & 0.1 & 24.5 & 4.7 & 12.9 & 29.6 & 2.2 \\
\hline
\end{tabular}

Quelle: SAKE (1995), eigene Berechnungen

Anmerkungen: Erwerbstätige Bevölkerung: Personen zwischen 16 und 64 Jahren

Einwanderungskohorten: Aufenthaltsdauer in Jahren

Wirtschaftszweige: 1 = Land-, Forstwirtschaft, 2 = Energie-/Wasserversorgung, Bergbau, 3 = Verarbeitende Produktion, $4=$ Baugewerbe, $5=$ HandeVGastgewerbe, $6=$ Ver . kehr/Nachrichten, $7=$ Banken/Versicherungen/Immobilien, $8=$ Sonstige Dienstleistungen (Gesundheitswesen, Erziehung, Forschung), 9 = Öffentliche Verwaltung

TAB. 9.6 verdeutlicht schliesslich, dass auch markante kohortenspezifische Unterschiede bestehen. Erwähnenswert ist insbesondere, dass von den älteren zu den jüngeren Einwanderungskohorten eine Verringerung der Beschäftigungsanteile im sekundären, industriellen Sektor zu verzeichnen ist. Dieses Ergebnis reflektiert zum einen Veränderungen in der schweizerischen Wirtschaftsstruktur, die während der vergangenen Jahrzehnte zu einer Vergrösserung des Dienstleistungs- auf

198 Von Bedeutung sind im diesem Zusammenhang insbesondere die gesetzlichen Beschränkungen zur selbständigen Erwerbstätigkeit der Ausländer (siehe Kapitel 2). 
Kosten des industriellen Sektors geführt haben. Zum anderen deutet es aber auch darauf hin, dass Einwanderer der älteren Einwanderungskohorten durch eine relativ geringe berufliche und sektorale Mobilität charakterisiert sind.

\subsubsection{ERWERBSBETEILIGUNG UND BESCHÄFTIGUNGSGRAD}

In diesem sowie im nachfolgenden Abschnitt steht die Diskussion der Beschäftigungsperformance im engeren Sinne im Vordergrund. Im speziellen wird auf die Erwerbsbeteiligung und Erwerbslosigkeit von Ausländern eingegangen. In TAB. 9.7 ist die Erwerbsbeteiligung von Männern und Frauen nach Nationalitätengruppen abgebildet. ${ }^{199}$ Um eine umfassende Evaluation der geschlechts-, nationalitäten- und kohortenspezifischen Unterschiede zu gewährleisten, werden ihre beiden Determinanten getrennt behandelt. Die Erwerbsbeteiligung setzt sich einerseits aus der Erwerbsquote zusammen, die den Anteil der Erwerbstätigen an den Erwerbspersonen misst, sowie andererseits aus dem Beschäftigungsgrad. Mit Blick auf Tab. 9.7 fallen vor allem die grossen geschlechtsspezifischen Unterschiede sowohl hinsichtlich der Erwerbsquoten als auch hinsichtlich des Beschäftigungsgrades auf, welche die traditionelle Rollenverteilung zwischen den beiden Geschlechtern reflektieren.

Tab. 9.7: Erwerbsbeteiligung und Beschäftigungsgrad nach Nationalitätengruppen (in Prozent)

\begin{tabular}{|l|c|ccc|c|ccc|}
\hline Nationalitä- & \multicolumn{4}{|c|}{ Beschäftigungsgrad (Männer) } & \multicolumn{3}{c|}{ Beschäftigungsgrad (Frauen) } \\
tengruppen & EQ & $<20$ & $\mathbf{2 0 - 6 9}$ & $\mathbf{7 0 - 1 0 0}$ & EQ & $<20$ & $\mathbf{2 0 - 6 9}$ & $\mathbf{7 0 - 1 0 0}$ \\
\hline Nordeuropa & 96.3 & & 1.9 & 98.1 & 72.3 & 6.0 & 30.0 & 64.0 \\
Südeuropa & 94.1 & & 1.8 & 98.2 & 75.3 & 4.6 & 30.4 & 65.0 \\
Nicht-Europa & 95.3 & & 3.6 & 96.4 & 71.6 & 2.6 & 23.0 & 74.4 \\
\hdashline Ausländer & 95.1 & & 2.2 & 97.8 & 73.6 & 4.6 & 28.5 & 66.9 \\
Schweizer & 90.8 & 0.8 & 3.4 & 95.8 & 68.7 & 8.2 & 35.3 & 56.5 \\
\hline
\end{tabular}

Quelle: SAKE (1995), eigene Berechnungen

Anmerkungen: $E Q=$ Erwerbsquote: Erwerbspersonen (Erwerbstätige und Erwerbslose gemäss Definition SAKE) / Erwerbsfähige Bevölkerung (Personen im Alter zwischen 16 und 64 Jahren)

Zur Aufschlüsselung der Nationalitätengruppen siehe TAB. 8.2

Der Vergleich zwischen den verschiedenen Nationalitätengruppen zeigt, dass sowohl bei den Männern als auch bei den Frauen hinsichtlich der Erwerbsquoten keine grossen Unterschiede bestehen. Daraus lassen sich jedoch keine Schlussfolgerungen über die Beschäftigungsperformance ableiten, da die Erwerbspersonen sowohl die Erwerbstätigen als auch die Erwerbslosen umfassen und sich daher keine Aussagen über das Gewicht der beiden Komponenten bei der Bestimmung

199 Die Unterschiede in den Ergebnissen zwischen ТАВ. 3.5 und TAB. 9.7 sind auf die divergierenden Definitionen der Erwerbsbeteiligung zurückzuführen (siehe auch Kapitel 8). 
der Erwerbsquote machen lassen. Wird demgegenüber jedoch lediglich die Erwerbstätigenquote ${ }^{200}$ betrachtet, so zeigt sich, dass diese sowohl bei den Männern als auch bei den Frauen für die Einwanderer aus Nordeuropa z.T. deutlich über jener der Einwanderer aus den süd- und nicht-europäischen Staaten liegt. ${ }^{201}$ Aufgrund der geringen Unterschiede in den Erwerbsquoten zwischen den drei Nationalitätengruppen implizieren tiefere Erwerbstätigenquoten daher höhere Erwerbslosenquoten (siehe Abschnitt 9.1.5). Dieses Ergebnis ist darauf zurückzuführen, dass Zuwanderer aus kulturell, sprachlich und geographisch verwandten Gebieten die geringsten Probleme beim Transfer von Humankapital besitzen und für sie somit eine vergleichsweise bessere Performance zu erwarten ist.

Der Vergleich zwischen In- und Ausländern zeigt, dass zwar erwartungsgemäss nationalitätenspezifische Unterschiede in den Erwerbsquoten bestehen, dass aber bei den Männern keine signifikanten Divergenzen im Beschäftigungsgrad bestehen. Mit Blick auf die Frauen fällt demgegenüber auf, dass der Anteil der teilzeiterwerbstätigen Schweizerinnen deutlich über jenem der Ausländerinnen liegt. In TAB. 9.8 fällt schliesslich die markant tiefere Erwerbsquote der jüngsten Einwanderungskohorte bei den Frauen auf, die u.a. darauf zurückzuführen ist, dass die Erwerbstätigkeit von Ausländern, die im Rahmen des Familiennachzugs eingewandert sind, gesetzlich beschränkt ist.

Tab. 9.8: Erwerbsbeteiligung und Beschäftigungsgrad nach Einwanderungskohorten (in Prozent)

\begin{tabular}{|l|c:ccc|c|ccc|}
\hline $\begin{array}{l}\text { Einwande- } \\
\text { rungskohorten }\end{array}$ & $\boldsymbol{E Q}$ & \multicolumn{3}{|c|}{$\begin{array}{c}\text { Beschäftigungsgrad (Männer) } \\
<2\end{array}$} & $\mathbf{2 0 - 6 9}$ & $\mathbf{7 0 - 1 0 0}$ & $\mathrm{EQ}$ & \multicolumn{3}{c|}{ Beschäftigungsgrad (Frauen) } & $\mathbf{2 0 - 6 9}$ & $\mathbf{7 0 - 1 0 0}$ \\
\hline$<1$ & 92.1 & & & 100 & 54.8 & & & 100 \\
$1-5$ & 94.4 & & 3.8 & 96.2 & 73.4 & 7.8 & 26.2 & 66.0 \\
$5-10$ & 99.0 & & 3.6 & 96.4 & 72.9 & 3.4 & 26.4 & 70.2 \\
$10-15$ & 98.9 & 1.8 & 98.2 & 75.3 & 2.0 & 34.4 & 63.7 \\
$15-20$ & 94.9 & & 1.0 & 99.0 & 78.3 & 4.5 & 23.1 & 72.3 \\
$>20$ & 92.3 & & 1.8 & 98.2 & 73.4 & 5.3 & 31.8 & 62.9 \\
\hline
\end{tabular}

Quelle: SAKE (1995), eigene Berechnungen

Anmerkungen: $E Q=$ Erwerbsquote: Erwerbspersonen (Erwerbstätige und Erwerbslose gemäss Definition SAKE)/Erwerbsfähige Bevölkerung (Personen im Alter zwischen 16 und 64 Jahren)

Einwanderungskohorten: Aufenthaltsdauer in Jahren

200 Die Erwerbstätigenquote wird im Rahmen dieser Studie als das Verhältnis von Erwerbstätigen zur erwerbsfähigen Bevölkerung im Alter zwischen 16 und 64 definiert.

201 Siehe GOLDER (1998) und GolDER/STRAUBHAAR (1998). 


\subsubsection{ERWERBSLOSIGKEIT}

Neben der Erwerbstätigkeit dient die Erwerbslosigkeit als zweites Kriterium zur Beurteilung der Beschäftigungsperformance im engeren Sinne. Analog zur Analyse der Erwerbstätigkeit werden die beiden Determinanten der Erwerbslosigkeit ebenfalls getrennt betrachtet. Die Höhe der Erwerbslosigkeit bestimmt sich einerseits aus dem Risiko erwerbslos zu werden (Betroffenheitsrisiko) sowie andererseits aus der Wahrscheinlichkeit, als Erwerbsloser wieder eine Stelle zu finden, d.h. der Dauer der Erwerbslosigkeit (Abgangswahrscheinlichkeit). ${ }^{202}$ Die Unterscheidung dieser beiden Faktoren ist wichtig, weil z.B. institutionelle Hemmnisse bei der Arbeitssuche zwar die Dauer der Erwerbslosigkeit der Einwanderer beeinflussen, nicht aber das Betroffenheitsrisiko. Analog dazu ist denkbar, dass höhere Erwerbslosenquoten der Ausländer im Konjunkturverlauf auf ein höheres Betroffenheitsrisiko zurückgeführt werden können, während die Erwerbslosigkeitsdauer vergleichsweise kurz ausfallen kann.

Zur Unterscheidung dieser beiden Komponenten werden in den folgenden Tabellen das Betroffenheitsrisiko, d.h. die Erwerbslosenquote, und die durchschnittliche Erwerbslosigkeitsdauer getrennt aufgeführt. ${ }^{203}$ In TAB. 9.9 finden sich die Erwerbslosenquoten sowie die Dauer der Erwerbslosigkeit nach Nationalitätengruppen gegliedert. In Übereinstimmung mit der Analyse zur Erwerbsbeteiligung lassen sich die Unterschiede zwischen TAB. 3.6 und TAB. 9.9 auf Divergenzen in den Definitionen der Erwerbs- und Arbeitslosenquoten zurückführen. ${ }^{204}$

Es zeigt sich, dass sowohl für Männer als auch Frauen wiederum substantielle nationalitätenspezifische Unterschiede in den Erwerbslosenquoten bestehen. Während sich aus dem Vergleich von TAB. 9.7 und 9.9 kein inverser Zusammenhang zwischen der Höhe der Erwerbsquoten und der Erwerbslosenquoten ableiten lässt, ist ein solcher deutlich erkennbar, wenn die Erwerbstätigenquoten und die Erwerbslosenquoten miteinander verglichen werden. ${ }^{205} \mathrm{Um}$ eine umfassende Evaluation der Erwerbslosigkeit zu gewährleisten, gilt es, wie erwähnt, neben der Erwerbslosenquote auch die Erwerbslosigkeitsdauer zu berücksichtigen.

Es zeigt sich, dass sowohl bei den Männern als auch bei den Frauen die Erwerbslosen mit der längsten Erwerbslosigkeitsdauer vorwiegend aus dem süd- bzw. nicht-europäischen Raum stammen. Einzig bei den Frauen aus Nordeuropa trifft dies nicht zu, wobei die detaillierte Analyse in TAB. A9.9 in Anhang 1 zeigt, dass dieses Ergebnis allein durch die weiblichen Zuwanderer aus Deutschland bedingt

202 Siehe Franz (1991).

203 Die Erwerbslosigkeitsdauer bezieht sich in diesem Zusammenhang allerdings nicht auf die vollendete Dauer einer Erwerbslosigkeitsperiode, sondern lediglich auf die Erwerbslosigkeitsdauer bis zum Erhebungszeitpunkt.

204 Siehe auch Abschnitt 8.4.

205 Siehe Golder (1998) und GoldER/STRAUBHAAR (1998). 
ist. Insgesamt resultiert somit die Schlussfolgerung, dass vor allem für Ausländer aus dem süd- und nicht-europäischen Raum die Chancen für eine Wiedereingliederung in den Arbeitsmarkt relativ schlecht stehen.

Tab. 9.9: Erwerbslosenquoten und Dauer der Erwerbslosigkeit nach Nationalitätengruppen (in Prozent)

\begin{tabular}{|c|c|c|c|c|c|}
\hline \multirow{2}{*}{$\begin{array}{l}\text { Nationalitäten- } \\
\text { gruppen }\end{array}$} & \multirow[t]{2}{*}{$E L Q$} & \multicolumn{4}{|c|}{ Erwerbslosigkeitsdauer (in Monaten) } \\
\hline & & $<3$ & $3-12$ & $12-24$ & $>24$ \\
\hline \multicolumn{6}{|l|}{ Männer } \\
\hline Nordeuropa & 2.4 & 46.7 & 21.6 & 31.6 & \\
\hline Sïdeuropa & 5.9 & 23.1 & 29.3 & 39.1 & 8.5 \\
\hline Nicht-Europa & 9.6 & 20.4 & 44.4 & 15.3 & 19.9 \\
\hline Ausländer & 5.6 & 25.0 & 33.1 & 29.3 & 12.6 \\
\hline Schweizer & 1.9 & 37.3 & 32.4 & 23.1 & 7.2 \\
\hline \multicolumn{6}{|l|}{ Frauen } \\
\hline Nordeuropa & 1.4 & & 76.1 & 4.1 & 19.8 \\
\hline Südeuropa & 8.9 & 26.3 & 33.1 & 33.1 & 7.5 \\
\hline Nicht-Europa & 11.5 & 30.0 & 37.9 & 5.1 & 27.0 \\
\hline Ausländer & 6.9 & 25.7 & 37.6 & 22.0 & 14.7 \\
\hline Schweizer & 3.0 & 25.4 & 36.6 & 18.2 & 19.8 \\
\hline
\end{tabular}

Quelle: SAKE (1995) eigene Berechnungen

Anmerkungen: $E L Q=$ Erwerbslosenquote: Erwerbslose (gemäss Definition SAKE) / Erwerbspersonen im Alter zwischen 16 und 64 (Erwerbstätige und Erwerbslose gemäss Definition SAKE)

Zur Aufschlüsselung der Nationalitätengruppen siehe TAB. 8.2

Neben den nationalitätenspezifischen bestehen auch wiederum geschlechtsspezifische Unterschiede sowohl hinsichtlich der Erwerbslosenquoten als auch hinsichtlich der Dauer der Erwerbslosigkeit. Schliesslich geht aus TAB. 9.9 hervor, dass sowohl bei den Männern als auch bei den Frauen die Erwerbslosenquoten der Schweizer deutlich unter jenen der Zuwanderer liegen. Hinsichtlich der Erwerbslosigkeitsdauer ist erkennbar, dass zwar die inländischen Männer nicht aber die inländischen Frauen ein vorteilhafteres Erwerbslosigkeitsprofil aufweisen.

Der Blick auf TAB. 9.10 zeigt, dass sowohl bei Männern als auch bei Frauen relativ grosse Divergenzen zwischen den einzelnen Einwanderungskohorten bestehen. Wie aus TAB. 8.6 und 8.7 hervorgeht, hängen diese Unterschiede mit der Verlagerung der Herkunftsländer und den damit verbundenen Veränderungen der 'Qualität' der Zuwanderer zusammen. Die geringe Erwerbslosenquote der jüngsten Einwanderungskohorte sowie die Unterschiede in den Erwerbslosenquoten zwischen Männern und Frauen lassen sich analog zu den Erwerbsquoten vor allem auf die bereits erwähnten ausländerrechtlichen Bestimmungen zurückzuführen. Schliesslich veranschaulicht TAB. 9.10, dass sich bei Erhebungen wie der SAKE, die auf 
Befragungen einzelner Personen oder Haushalte beruhen, Probleme aufgrund fehlerhafter Auskünfte einzelner Individuen nicht vollständig ausschliessen lassen. So ist es nicht möglich, dass Erwerbslose mit einer Aufenthaltsdauer von weniger als einem Jahr, mehr als 12 Monate erwerbslos sind. Dieser Fehler kann einerseits darauf beruhen, dass die befragten Personen entweder falsche Angaben zur Aufenthaltsdauer oder zur Erwerbslosigkeitsdauer gemacht haben. Durch die Struktur des Fragebogens wie auch durch das computerisierte Erhebungssystem werden solche Fehler jedoch minimiert.

Tab. 9.10: Erwerbslosenquoten und Dauer der Erwerbslosigkeit nach Einwanderungskohorten (in Prozent)

\begin{tabular}{|c|c|c|c|c|c|}
\hline \multirow{2}{*}{$\begin{array}{l}\text { Einwanderungs- } \\
\text { kohorten }\end{array}$} & \multirow[t]{2}{*}{$E L Q$} & \multicolumn{4}{|c|}{ Erwerbslosigkeitsdauer (in Monaten) } \\
\hline & & $<3$ & $3-12$ & $12-24$ & $>24$ \\
\hline \multicolumn{6}{|l|}{ Männer } \\
\hline$<1$ & 0.0 & & & & \\
\hline $1-5$ & 6.0 & 40.6 & 20.6 & 16.4 & 22.4 \\
\hline $5-10$ & 8.2 & 31.9 & 61.1 & 7.1 & \\
\hline $10-15$ & 6.4 & 31.3 & 38.8 & 5.1 & 24.8 \\
\hline $15-20$ & 4.0 & 14.5 & & 85.5 & \\
\hline$>20$ & 4.5 & 13.2 & 17.1 & 55.8 & 13.9 \\
\hline \multicolumn{6}{|l|}{ Frauen } \\
\hline$<1$ & 6.8 & & 85.6 & 14.4 & \\
\hline $1-5$ & 9.5 & 14.6 & 56.8 & & 28.6 \\
\hline $5-10$ & 5.2 & 42.9 & 29.6 & 11.8 & 11.8 \\
\hline $10-15$ & 8.0 & 44.7 & 30.2 & 21.8 & 27.8 \\
\hline $15-20$ & 8.3 & 44.4 & 55.6 & & \\
\hline$>20$ & 6.0 & 12.6 & 25.3 & 53.1 & 9.0 \\
\hline
\end{tabular}

Quelle: SAKE(1995), eigene Berechnungen

Anmerkungen: $E L Q=$ Erwerbslosenquote: Erwerbslose (gemäss Definition SAKE) / Erwerbspersonen im Alter zwischen 16 und 64 (Erwerbstätige und Erwerbslose gemäss Definition SAKE) Einwanderungskohorten: Aufenthaltsdauer in Jahren

\subsection{EMPIRISCHE ANALYSE DER ERWERBSLOSIGKEIT}

Im Rahmen dieses sowie des nachfolgenden Abschnitts steht die empirische Analyse der Beschäftigungsperformance im Vordergrund. Wie bereits einleitend erwähnt wurde, werden sowohl produktivitätsbezogene als auch diskriminierungsbedingte Faktoren in der Analyse berücksichtigt, wobei in diesem Abschnitt die Analyse der produktivitätsbezogenen Determinanten der Beschäftigungsperformance im Zentrum steht. Es gilt dabei vor allem die Frage zu beantworten, ob zwischen In- und Ausländern signifikante Unterschiede in den Erwerbstätigkeits- 
bzw. Erwerbslosigkeitsprofile bestehen und auf was diese Unterschiede allenfalls zurückzuführen sind.

Hierzu werden in der Literatur grundsätzlich drei verschiedene Messgrössen verwendet: erstens die Erwerbslosigkeitswahrscheinlichkeit, die über die Erwerbslosenquote approximiert wird, zweitens die Erwerbstätigkeitswahrscheinlichkeit, die über die Erwerbstätigenquote gemessen wird, sowie drittens die Partizipationswahrscheinlichkeit, die über die Erwerbsquote gemessen wird. Aufgrund der signifikanten Unterschiede in den Erwerbslosenquoten, der weitgehenden Übereinstimmung der Erwerbstätigenquoten zwischen In- und Ausländern sowie der Vermischung dieser beiden Grössen in der Erwerbsquote, erscheint es am sinnvollsten, die Erwerbslosigkeitswahrscheinlichkeit zur Messung der Beschäftigungsperformance zu verwenden. ${ }^{206}$

\subsubsection{MeTHODISCHE VORGEHENSWEISE}

Eine zentrale Zielsetzung der ökonomischen Theorie besteht in der Erklärung des Niveaus einer bestimmten abhängigen Variablen durch eine oder mehrere Einflussfaktoren. Die Entwicklung ökonometrischer Methoden hat sich schwerpunktmässig an dieser Zielsetzung orientiert. Das klassische ökonometrische Instrumentarium kann im Falle qualitativer Regressoren, sog. Dummy-Variablen, ohne weiteres übernommen werden. Dies trifft jedoch nicht zu, wenn es gilt, qualitative abhängige Variablen zu erklären. In diesem Fall besitzt die zu erklärende Variable $Y$ kein metrisches Messniveau, sondern diskrete Ausprägungen. Man spricht dann von sog. qualitativen Wahlhandlungsmodellen (qualitative response models), auf die sich die klassischen ökonometrischen Methoden nicht ohne weiteres übertragen lassen. Diese Modelle lassen sich in binomiale und multinomiale Wahlhandlungsmodelle unterteilen. Während im ersten Fall die zu erklärende Variable zwei diskrete Ausprägungen annehmen kann, z.B. die Entscheidung, Arbeit anzubieten oder nicht, besitzt die zu erklärende Variable im zweiten Fall mehr als zwei diskrete Ausprägungen, z.B. die Entscheidung für einen bestimmten Kandidaten in Parlamentswahlen. Die ökonometrische Analyse im Rahmen der vorliegenden Studie erfolgt über die Schätzung sog. binomialer Probit-Regressionen.

Im folgenden werden einige methodische Fragen erörtert, die sich bei der ökonometrischen Analyse der Erwerbslosigkeitswahrscheinlichkeit auf der Basis von Individualdaten ergeben. Die Analyse dieses Abschnitts gliedert sich wie folgt. ${ }^{207}$

206 Die Erwerbstätigkeitswahrscheinlichkeit ist jedoch im Rahmen der Analyse der Einkommensperformance in Kapitel 10 für die Berechnung der sog. Heckman-Korrektur von Bedeutung, die zur Korrektur des Stichproben-Auswahlfehlers verwendet wird.

207 Die folgenden Ausführungen beziehen sich weitgehend auf FRANZ (1991), BERNDT (1996) und GREENE (1995). 
Erstens werden einige methodische Ansätze zur Analyse qualitativer Wahlhandlungsmodell sowie damit zusammenhängende Probleme diskutiert. Zweitens wird in Abschnitt 9.2.1.2 die Modellspezifikation für die Probit-Regressionen erörtert.

\subsubsection{Methodische Ansätze und Probleme: Probit- und Logit-Modelle}

Zur ökonometrischen Modellierung von Wahlhandlungen wird die dichotome abhängige Variable $\mathrm{Y}$ betrachtet, die im Rahmen der vorliegenden Studie den Erwerbsstatus reflektiert. $Y=1$ bedeutet, dass die befragte Person erwerbslos ist und $Y=0$, dass sie entweder erwerbstätig oder nicht erwerbstätig ist. Wird davon ausgegangen, dass eine Reihe erklärender Variablen wie das Alter, die Schulausbildung oder das Geschlecht, die in einem Vektor x zusammengefasst sind, die Erwerbslosigkeitswahrscheinlichkeit beeinflussen, dann gilt

$$
\begin{aligned}
& \operatorname{Prob}(Y=1)=F\left(\beta^{\prime} x\right) \\
& \operatorname{Prob}(Y=0)=1-F\left(\beta^{\prime} x\right)
\end{aligned}
$$

wobei der Parametervektor $\beta$ den Einfluss von Veränderungen in den erklärenden Variablen $\mathrm{x}$ auf die Wahrscheinlichkeit misst. Es stellt sich in diesem Zusammenhang allerdings die Frage nach einem adäquaten Modell für die Rechthandseite von Gl. (9.1). Wird ein linearer Zusammenhang zwischen exogenen und endogenen Variablen unterstellt, so resultiert das sog. lineare Wahrscheinlichkeitsmodell (LPM)

$$
F(x, \beta)=\beta^{\prime} x .
$$

$\mathrm{Da} E[\mathrm{y}]=\mathrm{F}(\mathrm{x}, \beta)$ gilt, lässt sich das Regressionsmodell wie folgt formulieren

$$
\begin{aligned}
y & =E[y]+(y-E[y]) \\
& =\beta^{\prime} x+\varepsilon
\end{aligned}
$$

Das lineare Wahrscheinlichkeitsmodell ist jedoch mit einer Reihe von Problemen behaftet. Erstens ist die Störgrösse nicht normalverteilt. Da Y nur die Werte 0 und 1 annehmen kann, folgt der Störterm $\varepsilon$ einer Zweipunkteverteilung. Für die Schätzung des Parametervektors $\beta$ im multiplen Regressionsmodell mittels der $\mathbf{M e}$ thode der Kleinsten Quadrate (OLS-Methode) ist zwar keine Annahme über die Verteilung der Störgrösse notwendig, inferenzstatistische Konzepte, die über die Punktschätzung von $\beta$ hinausgehen, basieren jedoch i.d.R. auf der Annahme der Normalverteilung der Störgrösse, die zumindest approximativ gegeben sein sollte. Zweitens ist der Störterm $\varepsilon$ heteroskedastisch, da er von $\beta$ abhängt. Da $\beta^{\prime} x+\varepsilon$ entweder gleich null oder eins ist, entspricht $\varepsilon$ entweder $-\beta^{\prime} \mathrm{x}$ oder $1-\beta^{\prime} \mathrm{x}$ mit den Wahrscheinlichkeiten 1-F und F. Daraus geht hervor, dass

$$
\operatorname{Var}[\varepsilon]=\beta^{\prime} x\left(1-\beta^{\prime} x\right) \text {. }
$$


Dieses Problem liesse sich jedoch durch die Verwendung eines FGLS-Schätzers (Feasible Generalized Least Square Estimator) beheben. ${ }^{208}$ Das zentrale Problem hängt jedoch drittens damit zusammen, dass im Rahmen des linearen Wahrscheinlichkeitsmodells nicht gewährleistet ist, dass die systematische Komponente in Gl. (9.2) immer im Intervall $[0,1]$ liegt, so dass eine Interpretation als Wahrscheinlichkeit für das betrachtete Ereignis nicht mehr ohne weiteres möglich ist. Es wird daher i.a. auf die Verwendung des linearen Wahrscheinlichkeitsmodells verzichtet.

Aus diesem Grund wird eine Transformation benötigt, die sicherstellt, dass $\beta^{\prime} \mathbf{x}$ immer im Intervall $[0,1]$ liegt, unabhängig davon, welche Werte die exogenen Variablen annehmen. Im Hinblick auf die Analyse von Wahrscheinlichkeiten liegt es nahe, diese Transformation mit Hilfe einer Verteilungsfunktion vorzunehmen, die nur in diesem Intervall definiert ist. ${ }^{209}$ Diese Transformation liegt den Probit- und Logit-Modellen zugrunde. Beide Verfahren unterscheiden sich lediglich durch den Typ der Verteilungsfunktion. Während den Probit-Modellen eine Normalverteilung zugrundeliegt, basieren die Logit-Modelle auf einer logistischen Verteilung. Mit Ausnahme ihrer Randwerte unterscheiden sich die Dichtefunktionen beider Verteilungen im univariaten Bereich nur unwesentlich, so dass die Schätzergebnisse beim Logit- und Probit-Verfahren i.d.R. kaum differieren. Im Rahmen der vorliegenden Studie wird zur empirischen Analyse qualitativer Wahlhandlungsmodelle auf binäre Probit-Modelle zurückgegriffen.

Die Funktionsweise bzw. die wichtigsten Merkmale des Probit-Modells lassen sich mit Hilfe eines 'latenten Modells', eines sog. Indexfunktionsmodells, veranschaulichen. $\mathrm{Zu}$ diesem Zweck wird davon ausgegangen, dass eine 'latente', d.h. nicht-beobachtbare Variable $y^{*}$ und eine dichotome, d.h. nur zwei Ausprägungen annehmende Variable y, existieren. Es wird unterstellt, dass diese latente Indexvariable von einer Anzahl erklärender Variablen wie dem Alter, der Ausbildung oder der Arbeitserfahrung abhängt, so dass

$$
y^{*}=\beta^{\prime} x+\varepsilon .
$$

$\varepsilon$ folge einer Standardnormalverteilung, so dass $[\varepsilon \sim N(0,1)]$ gilt. Da der Einfluss der einzelnen Variablen nicht beobachtet werden kann, sondern lediglich die Tatsache, ob jemand erwerbslos ist oder nicht, kann davon ausgegangen werden, dass ein Wirtschaftssubjekt dann erwerbslos ist, wenn die Indexvariable einen gewissen Grenzwert überschreitet. Wird dieser Grenzwert zur Vereinfachung gleich null gesetzt, dann lässt sich die Beziehung zwischen der unbeobachtbaren Indexvariablen $\mathrm{y}^{*}$ und der beobachtbaren Variablen y durch

\section{Siehe GREENE (1995: Kapitel 14).}

209 Eine Verteilungsfunktion gibt die Wahrscheinlichkeit an, dass eine Zufallsvariable kleiner oder gleich einem bestimmten Wert dieser Variable ist. 


$$
y=\left\{\begin{array}{l}
1 \text { falls } y^{*}>0 \\
0 \text { falls } y^{*} \leq 0
\end{array}\right.
$$

ausdrücken. In dieser Formulierung wird $\beta^{\prime} x$ als Indexfunktion bezeichnet. Die Wahrscheinlichkeit, dass ein Individuum erwerbslos ist, d.h. $y=1$, ist dann durch

$$
\begin{aligned}
\operatorname{Pr}\left(\mathrm{y}^{*}>0\right) & =\operatorname{Pr}\left(\beta^{\prime} \mathrm{x}+\varepsilon>0\right) \\
& =\operatorname{Pr}\left(\varepsilon>-\beta^{\prime} \mathrm{x}\right)
\end{aligned}
$$

und im Falle einer symmetrischen Wahrscheinlichkeitsverteilung durch

$$
\begin{aligned}
\operatorname{Pr}\left(\mathrm{y}^{*}>0\right) & =\operatorname{Pr}\left(\varepsilon<\beta^{\prime} \mathrm{x}\right) \\
& =\mathrm{F}\left(\beta^{\prime} \mathrm{x}\right)
\end{aligned}
$$

gegeben. Ein analoges Modell resultiert, wenn die latente Variable als Nutzenindex interpretiert wird. In diesem Zusammenhang wird unterstellt, dass für die beiden Alternativen stochastische Nutzenindizes $U_{1}$ und $U_{2}$ bestehen, wobei die Erwartungswerte dieser Indizes wiederum von einem Vektor erklärender Variablen abhängen. Somit lässt sich die Wahrscheinlichkeit, dass der Nutzen von Alternative 1 grösser ist als jener von Alternative 2, als $\mathrm{y}^{*}=\mathrm{U}_{1}-\mathrm{U}_{2}$ bzw. $\mathrm{y}^{*}>0$ interpretieren. Dieser Ansatz, der von der Maximierung des Zufalls-Nutzens (random utility maximisation) ausgeht, ist für die 'Discrete-Choice'-Theorie von zentraler Bedeutung. ${ }^{210}$

Sowohl das Probit- als auch das Logit-Modell können nicht unter Verwendung der Methode der Kleinsten Quadrate geschätzt werden, weil es sich um nicht-lineare Beziehungen handelt. Alternativ gelangt daher die Maximum-Likelihood-Methode (MLE-Methode) als Schätzverfahren zur Anwendung. Die geschätzten Parameter des Probit-Modells sind dabei, wie in allen nicht-linearen Modellen, nicht notwendigerweise identisch mit den marginalen Effekten. Der Einfluss von Veränderungen in den erklärenden Variablen auf die Wahrscheinlichkeit eines betrachteten Ereignisses ergeben sich im Probit-Modell aus den partiellen Ableitungen wie folgt:

$$
\begin{aligned}
\frac{\partial E[y]}{\partial x} & =\left\{\frac{d F\left(\beta^{\prime} x\right)}{d\left(\beta^{\prime} x\right)}\right\} \beta \\
& =f\left(\beta^{\prime} x\right) \beta
\end{aligned}
$$

wobei $f($.) die Dichtefunktion der Standardnormalverteilung bezeichnet. Durch das Vorzeichen der Koeffizienten allein ist lediglich die Richtung determiniert, in die sich die Wahrscheinlichkeit bei bestimmten Impulsen der exogenen Variablen

210 Siehe BEN AKIVA/LERMAN (1991). 
verändert. Das Ausmass der Veränderung hängt zusätzlich von der Steigung der Verteilungsfunktion der Normalverteilung im Punkt $\beta$ 'x ab und verdeutlicht damit einen wichtigen Unterschied zum LPM. Je steiler die Verteilungsfunktion beim aktuellen Niveau verläuft, desto grösser ist der Effekt einer Veränderung der exogenen Variablen auf die Wahrscheinlichkeit des betrachteten Ereignisses.

Ein zweiter Punkt betrifft die Messung der Anpassungsgüte des Regressionsmodells. Diese erfolgt in klassischen OLS-Schätzungen i.d.R. über das sog. (korrigierte) Bestimmtheitsmass (adj.-R $\boldsymbol{R}^{2}$ ), das definiert ist als das Verhältnis der durch die Regression erklärten Varianz zur Gesamtvarianz der endogenen Variablen, korrigiert um die Zahl der Freiheitsgrade. ${ }^{211} \mathrm{Da}$ sich ein analoges Bestimmtheitsmass für binomiale Wahlhandlungsmodelle nicht berechnen lässt, wurden verschiedene alternative Gütekriterien für Probit- und Logit-Modelle entwickelt. ${ }^{212}$ Das am häufigsten verwendete Gütemass wird, aufgrund der Ähnlichkeit zum Bestimmtheitsmass in OLS-Schätzungen, als Pseudo- $\boldsymbol{R}^{2}$ bezeichnet. Es handelt sich dabei um den Likelihood-Ratio Index

$$
\mathrm{PR}^{2}=1-\frac{\ln \left(\mathrm{L}^{*}\right)}{\ln \left(\mathrm{L}_{0}^{*}\right)},
$$

wobei $\ln \left(\mathrm{L}^{*}\right)$ die unrestringierte und $\ln \left(\mathrm{L}_{0}^{*}\right)$ die restringierte Log-LikelihoodFunktion bezeichnen. Ein wachsendes Pseudo- $\mathbf{R}^{2}$ impliziert zwar grundsätzlich eine Verbesserung der Modellanpassung, "... doch besitzen die Werte des Pseudo$\mathbf{R}^{2}$ nicht die anschauliche Interpretation, die dem Determinationskoeffizienten beim multiplen Regressionsmodell zukommt."213

\subsubsection{Modellspezifikation}

Nach der allgemeinen Diskussion von dichotomen Wahlhandlungsmodellen steht im folgenden die Modellspezifikation im Vordergrund. Wie bereits einleitend erwähnt wurde, dient die Erwerbslosigkeitswahrscheinlichkeit als Messgrösse für die Beschäftigungsperformance der In- und Ausländer. Die Modellspezifikation, d.h. die Operationalisierung der Determinanten der Erwerbslosigkeit, folgt dabei den Erkenntnissen der Arbeitsmarkttheorie.

In Anlehnung an die vorangegangene Diskussion der Indexfunktionsmodelle wird die Erwerbslosigkeitswahrscheinlichkeit als endogene Variable durch die dichotome Variable UNEMPL approximiert, die einen Wert von 1 annimmt, wenn die betrachtete Person erwerbslos ist und einen Wert von 0, wenn die Person er-

211 Für eine kritische Diskussion dieses Masses siehe KENNEDY (1996).

212 Eine Diskussion alternativer Gütemasse findet sich in AMEMIYA (1981), VEAU/ZMMERMANN (1992) und WINDMEUER (1995).

213 ECKEY/KOSFELD/DREGER (1995: 179). 
werbstätig ist. ${ }^{214}$ Auf aggregierter Ebene lässt sich daraus die Erwerbslosenquote berechnen, die dem Verhältnis der Erwerbslosen zu den Erwerbspersonen entspricht, wobei letztere die Erwerbslosen und die Erwerbstätigen umfassen. Analog zur deskriptiven Analyse umfasst die nachfolgende Analyse wiederum lediglich Personen im Alter zwischen 16 und 64 Jahren.

Nach der Diskussion der endogenen Variablen, stehen im folgenden die exogenen Variablen, d.h. die Determinanten der Erwerbslosigkeit im Vordergrund. Als erste wichtige Bestimmungsgrösse ist das Alter $(\boldsymbol{A G E})$ zu nennen. Aufgrund der durch die Querschnittsanalyse bedingten Vermischung der Dauer- und Risikokomponente ist der Effekt, den das Alter auf die Erwerbslosigkeitswahrscheinlichkeit hat, allerdings nicht eindeutig. Während für Jugendliche das Risiko, erwerbslos zu werden, im Vergleich zu den älteren Erwerbstätigen höher ist, nimmt die Erwerbslosigkeitsdauer mit zunehmendem Alter zu. ${ }^{215}$ Im Rahmen von Querschnittsbetrachtung ist allerdings zu vermuten, dass ältere Erwerbslose aufgrund der durchschnittlich längeren Erwerbslosigkeitsperioden mit grösserer Wahrscheinlichkeit in der Stichprobe vertreten sind, so dass eher mit einem positiven Effekt zunehmenden Alters auf die Erwerbslosigkeitswahrscheinlichkeit zu rechnen ist.

Als zweite personenspezifische Variable wird das Qualifikationsprofil berücksichtigt, das über die Anzahl Schuljahre (SCH) approximiert wird. Diese Variable ergibt sich gemäss TAB. 9.11 aus der Umrechnung der im Rahmen der SAKE erhobenen höchsten abgeschlossenen Ausbildung. ${ }^{216}$ Hinsichtlich des Qualifikationsprofils ist von einem negativen Zusammenhang zur Erwerbslosigkeitswahrscheinlichkeit auszugehen, da für gering Qualifizierte sowohl das Risiko als auch die Dauer der Erwerbslosigkeit i.a. höher ausfällt als für höher Qualifizierte. Es ist allerdings nicht auszuschliessen, dass der Ausbildungseffekt nicht signifikant ist, da allen Personen gemäss TAB. 9.11 mindestens eine Ausbildungsdauer von 7 Jah-

214 Als 'unfreiwillig' erwerbslos gilt eine Person i.a. dann, wenn sie zum herrschenden Lohnniveau keine Beschäftigung findet. Alternativ dazu handelt es sich bei den 'freiwillig' Erwerbslosen um Personen, die zu den marktgängigen Bedingungen nicht bereit sind zu arbeiten. Eine Unterscheidung dieser beiden Definitionen erweist sich in der Praxis jedoch als schwierig.

215 Siehe FRANZ (1991).

216 Diese Umrechnung ist jedoch nicht unproblematisch, da die in TAB. 9.11 verwendeten Umrechnungsfaktoren auf der normalen Zeitspanne basieren, die zur Erreichung eines bestimmten Abschlusses notwendig ist. Vernachlässigt bleiben somit sowohl interindividuelle Unterschiede in den Ausbildungszeiten als auch Qualitätsunterschiede in der Schulausbildung. Dieser Umstand erweist sich insbesondere für die Berechnung der Ausbildungsdauer der Ausländer als problematisch, da sich nicht nur die Zuordnung der ausländischen Abschlüsse auf die in TAB. 9.11 verwendeten Kategorien als schwierig erweisen kann, sondern weil die Verwendung identischer Umrechnungsfaktoren für In- und Ausländer international unterschiedlichen Bildungssystemen nicht Rechnung zu tragen vermag. 
ren zugewiesen wird und Bildungsunterschieden daher eventuell zu wenig stark Rechnung getragen werden kann. ${ }^{217}$

Tab. 9.11: Berechnung der Anzahl Schuljahre (SCH)
\begin{tabular}{|l|c|}
\hline Höchste abgeschlossene Ausbildung & Anzahl Schuljahre (SCH) \\
\hline Kein Abschluss & 7 \\
Obligatorische Grundschule & 8 \\
Anlehre & 8.75 \\
Berufslehre & 9.5 \\
Vollzeitberufsschule & 11 \\
Diplommittelschule & 10.5 \\
Matura & 12.5 \\
Meisterdiplom & 11 \\
Technikerschule & 11.5 \\
Höhere Fachschule/Technikum & 15.5 \\
Universität/Hochschule & 17.5 \\
Andere Ausbildung & 8 \\
\hline
\end{tabular}

Quelle: Bundesamt für Statistik

Ein nicht-signifikanter Effekt der Ausbildungsvariablen lässt sich mit Blick auf die Ausländer aber auch damit begründen, dass diese nicht in der Lage sind, das im Herkunftsland erworbene Humankapital vollständig ins Zielland zu transferieren, so dass zwischen der Erwerbslosigkeitswahrscheinlichkeit und der Ausbildung kein oder lediglich ein schwacher Zusammenhang besteht. Schliesslich kann ein nicht-signifikanter oder negativer Effekt auch ein Indiz für 'Mismatch'-Erwerbslosigkeit sein. Von qualifikationsbedingter Mismatch-Erwerbslosigkeit wird dann gesprochen, wenn zwischen den Qualifikationsprofilen der Arbeitsanbieter und den Qualifikationsanforderungen der Arbeitsnachfrager keine Übereinstimmung besteht. Diese Form der Erwerbslosigkeit dient i.d.R. als Indikator für das Ausmass der strukturellen Komponente der Erwerbslosigkeit.

Neben diesen personenspezifischen Faktoren wird mit dem logarithmierten monatlichen Haushaltsresidualerwerbseinkommen $\left(\boldsymbol{L} \boldsymbol{N}_{-} \boldsymbol{H} H I N C\right)$ auch eine Variable berücksichtigt, die der Haushaltsstruktur Rechnung trägt. Das monatliche Haushaltsresidualeinkommen berechnet sich aus dem monatlichen Totalerwerbseinkommen des Haushalts abzüglich des monatlichen Erwerbseinkommens der Zielperson. Der Berücksichtigung dieser Variablen liegt die Überlegung zugrunde,

217 Diesem Umstand liesse sich allenfalls durch die Verwendung einer Dummy-Variablen für gelernte und ungelernte Arbeitskräfte Rechnung tragen. 
dass die Erwerbslosigkeitswahrscheinlichkeit auch von der wirtschaftlichen Lage des Haushalts beeinflusst wird. Einerseits erhöhen sich mit zunehmendem Haushaltsresidualeinkommen die Opportunitätskosten der Arbeit und damit ebenfalls das Risiko, erwerbslos zu werden, andererseits impliziert die Suchtheorie, dass sich mit steigendem Haushaltseinkommen die Kosten der Arbeitssuche verringern, was eine Verlängerung der Erwerbslosigkeitsdauer zur Folge haben kann. Insgesamt ist daher mit einem positiven Effekt des Haushaltsresidualeinkommens auf die Erwerbslosigkeitswahrscheinlichkeit zu rechnen.

Neben diesen mikroökonomischen Faktoren spielt auch die wirtschaftliche Lage als makroökonomische Grösse eine wichtige Rolle bei der Erklärung von Erwerbslosigkeit. Als Indikator für die wirtschaftliche Lage dienen die kantonalen Arbeitslosenquoten (UNPL). Es ist anzunehmen, dass zwischen der Höhe kantonaler Arbeitslosenquoten und den individuellen Erwerbslosigkeitswahrscheinlichkeiten ein positiver Zusammenhang besteht. Abschliessend werden in den ProbitRegressionen der Ausländer zwei Dummy-Variablen für Nordeuropäer (NTHEU_IC) und Südeuropäer (STHEU) verwendet, wobei die Nicht-Europäer (OTHCT) als Basiskategorie dienen, um Unterschieden in den Erwerbslosigkeitswahrscheinlichkeiten zwischen den drei Nationalitätengruppen Rechnung zu tragen. Eine Zusammenfassung der Modellspezifikation bzw. Definition der zur Schätzung der Erwerbslosigkeitsgleichung verwendeten Variablen findet sich in TAB. 9.12.

\section{Tab. 9.12: Modellspezifikation: Erwerbslosigkeitswahrscheinlichkeit}

\begin{tabular}{|c|c|c|c|}
\hline $\begin{array}{l}\text { Variablen- } \\
\text { name }\end{array}$ & Definition & $\begin{array}{l}\text { Variablen- } \\
\text { name }\end{array}$ & Definition \\
\hline UNEMPL & $\begin{array}{l}\text { Erwerbslosigkeitswahr- } \\
\text { scheinlichkeit }\end{array}$ & UNPL & $\begin{array}{l}\text { Kantonale } \\
\text { Arbeitslosenquoten }\end{array}$ \\
\hline$A G E$ & Alter in Jahren & NTHEU_IC & $\begin{array}{l}\text { Dummy-Variable für } \\
\text { Nordeuropäer }\end{array}$ \\
\hline $\mathrm{SCH}$ & Anzahl Schuljahre & STHEU & $\begin{array}{l}\text { Dummy-Variable für } \\
\text { Südeuropäer }\end{array}$ \\
\hline$L N \_H H I N C$ & $\begin{array}{l}\text { Logarithmiertes monatliches } \\
\text { Haushaltsresidualeinkommen }\end{array}$ & & \\
\hline
\end{tabular}

Quelle: Eigene Darstellung

\subsubsection{EMPIRISCHE ERGEBNISSE}

Nach der Diskussion des Modellrahmens folgen in diesem Abschnitt die empirischen Ergebnisse. Als Einleitung werden in TAB. 9.13 die deskriptiven Statistiken zu den Variablen des Probit-Modells präsentiert. In Übereinstimmung mit den Ergebnissen der deskriptiven Analyse in Abschnitt 9.1 zeigt sich, dass die Erwerbslosigkeitswahrscheinlichkeit der Ausländer deutlich über jener der Inländer liegt 
und jene der Frauen, zumindest bei den Ausländern, klar über jener der Männer. Es gilt nachfolgend zu überprüfen, ob diese Ergebnisse auf die in TAB. 9.13 dargestellten Unterschiede in den erklärenden Variablen zurückzuführen sind.

Tab. 9.13: Deskriptive Statistiken zur Beschäftigungsperformance

\begin{tabular}{|l|c|c|c|c|}
\hline Variablen & $\begin{array}{c}\text { Inländische } \\
\text { Männer }\end{array}$ & $\begin{array}{c}\text { Inländische } \\
\text { Frauen }\end{array}$ & $\begin{array}{c}\text { Ausländische } \\
\text { Männer }\end{array}$ & $\begin{array}{c}\text { Ausländische } \\
\text { Frauen }\end{array}$ \\
\hline Stichprobengrösse & 6799 & 5373 & 939 & 640 \\
\hdashline UNEMPL & 0.017 & 0.026 & 0.060 & 0.061 \\
AGE & 39.10 & 38.35 & 41.95 & 39.18 \\
SCH & $(11.50)$ & $(11.48)$ & $(10.42)$ & $(9.97)$ \\
LN_HHINC & 10.99 & 10.32 & 10.50 & 9.98 \\
& $(2.73)$ & $(2.23)$ & $(3.22)$ & $(2.93)$ \\
UNPL & 4.19 & 5.54 & 4.66 & 5.47 \\
& $(3.96)$ & $(4.01)$ & $(3.85)$ & $(3.90)$ \\
NTHEU_IC & 4.02 & 4.10 & 4.77 & 4.79 \\
STHEU & $(1.82)$ & $(1.83)$ & $(1.92)$ & $(1.97)$ \\
& & & 0.31 & 0.35 \\
& & & & \\
\hline
\end{tabular}

Quelle: SAKE (1995), eigene Berechnungen

Anmerkungen: Für stetige Variablen sind die Standardfehler in Klammern angegeben

Im folgenden werden die Ergebnisse der Probit-Schätzungen diskutiert. Im Rahmen von LR-Tests (Likelihood-Ratio Test) auf Parameterstabilität wurde dabei vorgängig geprüft, ob das Probit-Modell für In- und Ausländer (bzw. für Männer und Frauen) getrennt geschätzt werden muss oder ob Schätzungen für die gepoolten Stichproben zulässig sind. ${ }^{218}$ Obschon aus TAB. 9.14 hervorgeht, dass eine getrennte Analyse nicht notwendig ist, werden die Determinanten der Erwerbslosigkeitswahrscheinlichkeit für in- und ausländische Männer und Frauen, analog zur Präsentation der deskriptiven Statistiken, getrennt untersucht.

218 Der LR-Test wird im Rahmen der Analyse der Erwerbslosigkeitswahrscheinlichkeit verwendet, da die Schätzung der Probit-Regressionen unter Verwendung der MLE-Methode erfolgte. Im Rahmen von OLS-Schätzung bei der Analyse der Einkommensperformance werden demgegenüber zur Überprüfung der Parameterstabilität sog. 'Chow-Tests' durchgeführt (siehe Abschnitt 10.2). 
Tab. 9.14: LR-Tests auf Parameterstabilität

\begin{tabular}{|l|c|c|}
\hline & Teststatistik & Tabellenwert \\
\hline $\begin{array}{l}\text { Schweizer - } \\
\text { Ausländer } \\
\text { Schweizer (Männer) - } \\
\begin{array}{l}\text { Schweizer (Frauen) } \\
\text { Ausländer (Männer) - } \\
\text { Ausländer(Frauen) }\end{array}\end{array}$ & 3.92 & 11.07 \\
\hline
\end{tabular}

Quelle: SAKE (1995), eigene Berechnungen

Anmerkungen: Der Likelihood-Ratio Test folgt einer $\chi^{2}$-Verteilung, wobei die Zahl der Freiheitsgrade der Zahl der Restriktionen entspricht. Die Tabellenwerte sind auf dem Signifikanzniveau von 5 Prozent ausgewiesen

In TAB. 9.15 finden sich die Schätzergebnisse der Probit-Regression für die inländischen Männer und Frauen. Es zeigt sich, dass bei den Männern ein signifikant negativer Zusammenhang zwischen dem Alter und der Erwerbslosigkeitswahrscheinlichkeit besteht. Dieses Ergebnis lässt sich dahingehend interpretieren, dass für Männer mit zunehmendem Alter die Erwerbslosigkeitswahrscheinlichkeit sinkt. Dies impliziert, dass den Ausführungen des vorangegangenen Abschnitts folgend, dem Risiko bei der Erklärung der Erwerbslosigkeit eine grössere Bedeutung zukommt als der Dauer. Bei den inländischen Frauen ist der Effekt des Alters demgegenüber nicht signifikant. Dieses Ergebnis kann darauf zurückgeführt werden, dass sich die beiden gegenläufigen Effekte des Risikos und der Dauer gegenseitig aufheben.

Ein negativer Zusammenhang zur Erwerbslosigkeit besteht auch hinsichtlich des Qualifikationsprofils, wobei dieser Effekt nur für Frauen signifikant ist. Ein höheres Qualifikationsniveau verringert erwartungsgemäss die Wahrscheinlichkeit, erwerbslos zu werden. Die Insignifikanz der Ausbildungsvariablen bei den Männern impliziert, dass die Erwerbslosigkeitswahrscheinlichkeit nicht vom Ausbildungsniveau beeinflusst wird. Es ist denkbar, dass dieses Ergebnis dadurch bedingt ist, dass ein Mismatch zwischen den Qualifikationen der Arbeitsanbieter und den Anforderungsprofilen der Arbeitsnachfrager besteht, so dass ein höheres Qualifikationsprofil nicht notwendigerweise eine Verringerung der Erwerbslosigkeitswahrscheinlichkeit zur Folge hat.

Erwartungsgemäss besteht demgegenüber ein signifikant positiver Zusammenhang zwischen der Erwerbslosigkeit und dem Haushaltsresidualeinkommen sowie der regionalen wirtschaftlichen Lage. Im ersten Fall ist dies vor allem darauf zurückzuführen, dass sich nicht nur die Opportunitätskosten der Arbeit für Erwerbstätige erhöhen und damit das Erwerbslosigkeitsrisiko steigt, sondern dass sich aufgrund gesunkener Suchkosten auch die Suchintensität der Erwerbslosen verlangsamt. Im zweiten Fall ist ebenfalls davon auszugehen, dass eine Verschlechterung der wirt- 
schaftlichen Lage sowohl ein erhöhtes Risiko als auch eine Verlängerung der Dauer der Erwerbslosigkeit zur Folge hat.

Tab. 9.15: Probit-Regressionen zur Erwerbslosigkeitswahrscheinlichkeit, Schweizer

\begin{tabular}{|c|c|c|c|c|}
\hline \multirow[t]{2}{*}{ Variablen } & \multicolumn{2}{|c|}{ Männer } & \multicolumn{2}{|c|}{ Frauen } \\
\hline & $\begin{array}{c}\text { Schätzwert } \\
\text { (abs. } t \text {-Werte) }\end{array}$ & $\begin{array}{c}\text { Marginale } \\
\text { Effekte }\end{array}$ & $\begin{array}{c}\text { Schätzwert } \\
\text { (abs. } t \text {-Werte) }\end{array}$ & $\begin{array}{c}\text { Marginale } \\
\text { Effekte }\end{array}$ \\
\hline $\begin{array}{l}\text { Stichproben- } \\
\text { grösse }\end{array}$ & \multicolumn{2}{|c|}{6799} & \multicolumn{2}{|c|}{5373} \\
\hline CONSTANT & $\begin{array}{r}-2.697 * * * \\
(11.51)\end{array}$ & -0.0665 & $\begin{array}{r}-2.303^{* * *} \\
(8.47)\end{array}$ & -0.1025 \\
\hline$A G E$ & $\begin{array}{r}-0.009 * * * \\
(2.68)\end{array}$ & -0.0002 & $\begin{array}{r}-0.004 \\
(1.26)\end{array}$ & -0.0002 \\
\hline $\mathrm{SCH}$ & $\begin{array}{r}-0.018 \\
(1.21)\end{array}$ & -0.0004 & $\begin{array}{r}-0.053^{* * * *} \\
(2.69)\end{array}$ & -0.0024 \\
\hline LN_HHINC & $\begin{array}{r}0.113 * * * \\
(8.30)\end{array}$ & 0.0028 & $\begin{array}{r}0.092 * * * \\
(6.80)\end{array}$ & 0.0041 \\
\hline UNPL & $\begin{array}{r}0.106 * * * \\
(4.96)\end{array}$ & 0.0026 & $\begin{array}{r}0.100^{* * * *} \\
(4.92)\end{array}$ & 0.0045 \\
\hline $\log -L$ & \multicolumn{2}{|c|}{-523.153} & \multicolumn{2}{|c|}{-600.047} \\
\hline Pseudo- $R^{2}$ & \multicolumn{2}{|c|}{0.251} & \multicolumn{2}{|c|}{0.234} \\
\hline
\end{tabular}

Quelle: SAKE (1995), eigene Berechnungen

Anmerkungen: * bedeutet ein Signifikanzniveau von 10 Prozent, ** bedeuten ein Signifikanzniveau von 5 Prozent, *** bedeuten ein Signifikanzniveau von 1 Prozent

In TAB. 9.16 finden sich die Schätzergebnisse für die ausländischen Männer und Frauen. Ein wesentlicher Unterschied zu den Schätzergebnissen in TAB. 9.15 lässt sich hinsichtlich des Effektes der Altersvariablen beobachten, der für die ausländischen Männer signifikant positiv ist, während er für die inländischen Männer signifikant negativ ausfällt. Dieses Ergebnis lässt sich darauf zurückführen, dass im Rahmen von Querschnittsbetrachtungen Langzeiterwerbslose mit einer höheren Wahrscheinlichkeit in der Stichprobe vertreten sind, sowie dass ältere Personen i.d.R. eine längere Erwerbslosigkeitsdauer aufweisen als jüngere Personen. ${ }^{219}$

Mit Blick auf die ausländischen Frauen zeigt sich, dass der Koeffizient der Ausbildungsvariablen nicht signifikant ist. Analog zur vorangegangenen Erklärung für die inländischen Männer kann dies durch einen Mismatch zwischen Qualifikations- und Anforderungsprofilen bedingt sein. Zusätzlich liegt mit Blick auf Kapitel 5 die Überlegung nahe, dass aufgrund der unvollständigen Transferierbarkeit des im Ausland erworbenen Humankapitals die Qualifikationsprofile der Auslän-

219 Diese Überlegungen decken sich mit den Ergebnissen der TAB. 9.10 und 9.11. 
der nur bedingt einen positiven Einfluss auf die Beschäftigungsperformance besitzen.

Die Berücksichtigung nationalitätenspezifischer Unterschiede in TAB. 9.16 zeigt schliesslich, dass Zuwanderer aus Nordeuropa mit der geringsten Wahrscheinlichkeit und Zuwanderer aus dem nicht-europäischen Raum mit der grössten Wahrscheinlichkeit erwerbslos sind. Diese Ergebnisse decken sich mit jenen der deskriptiven Analyse in Abschnitt 9.1, aus der hervorging, dass die Nordeuropäer die vergleichsweise vorteilhaftesten und die Nicht-Europäer die unvorteilhaftesten individuellen Charakteristika besitzen.

Tab. 9.16: Probit-Regressionen zur Erwerbslosigkeitswahrscheinlichkeit, Ausländer

\begin{tabular}{|c|c|c|c|c|}
\hline \multirow[t]{2}{*}{ Variablen } & \multicolumn{2}{|c|}{ Männer } & \multicolumn{2}{|c|}{ Frauen } \\
\hline & $\begin{array}{c}\text { Schätzwert } \\
\text { (abs. } t \text {-Werte) }\end{array}$ & $\begin{array}{c}\text { Marginale } \\
\text { Effekte }\end{array}$ & $\begin{array}{c}\text { Schätzwert } \\
\text { (abs.t-Werte) }\end{array}$ & $\begin{array}{c}\text { Marginale } \\
\text { Effekte }\end{array}$ \\
\hline $\begin{array}{l}\text { Stichproben- } \\
\text { grösse }\end{array}$ & \multicolumn{2}{|c|}{939} & \multicolumn{2}{|c|}{640} \\
\hline CONSTANT & $\begin{array}{r}-2.977 * * * \\
(6.41)\end{array}$ & -0.2497 & $\begin{array}{r}-1.621 * * * \\
(2.74)\end{array}$ & -0.1516 \\
\hline$A G E$ & $\begin{array}{r}0.013^{*} \\
(1.93)\end{array}$ & 0.0011 & $\begin{array}{r}-0.006 \\
(0.64)\end{array}$ & -0.0005 \\
\hline $\mathrm{SCH}$ & $\begin{array}{r}-0.005 \\
(0.21)\end{array}$ & -0.0004 & $\begin{array}{r}-0.029 \\
(0.80)\end{array}$ & -0.0027 \\
\hline$L N \_H H I N C$ & $\begin{array}{r}0.110 * * * \\
(4.78)\end{array}$ & 0.0093 & $\begin{array}{r}0.081 * * * \\
(2.83)\end{array}$ & 0.0076 \\
\hline UNPL & $\begin{array}{r}0.119 * * * \\
(2.97)\end{array}$ & 0.0100 & $\begin{array}{l}0.073 \\
(1.61)\end{array}$ & 0.0068 \\
\hline NTHEU_IC & $\begin{array}{r}-0.540 * * * \\
(2.69)\end{array}$ & -0.0453 & $\begin{array}{r}-0.593 * * \\
(2.29)\end{array}$ & -0.0554 \\
\hline STHEU & $\begin{array}{r}-0.455^{* * * *} \\
(2.55)\end{array}$ & -0.0382 & $\begin{array}{r}-0.337 * \\
(1.65)\end{array}$ & -0.0316 \\
\hline $\log -L$ & -186 & & -134 & \\
\hline Pseudo- $R^{2}$ & & & & \\
\hline
\end{tabular}

Quelle: SAKE (1995), eigene Berechnungen

Anmerkungen: * bedeutet ein Signifikanzniveau von 10 Prozent, ** bedeuten ein Signifikanzniveau von 5 Prozent, *** bedeuten ein Signifikanzniveau von 1 Prozent

\subsection{EMPIRISCHE ANALYSE DER ARBEITSMARKSEGMENTATION}

Nachdem im vorangegangenen Abschnitt produktivitätsbezogene Faktoren zur Erklärung der Beschäftigungsperformance im Vordergrund standen, werden im Rahmen der nachfolgenden Ausführungen diskriminierungsbedingte Faktoren erörtert. Im speziellen geht es darum, das Ausmass der Arbeitsmarktsegmentation 
zu analysieren. In einem ersten Schritt wird auf die methodische Vorgehensweise eingegangen, in einem zweiten Schritt folgt eine empirische Analyse der Arbeitsmarktsegmentation.

\subsubsection{METHODISCHE VORGEHENSWEISE}

Die theoretischen Ausführungen zur Diskriminierung auf dem Arbeitsmarkt in Kapitel 6 haben gezeigt, dass eine Form der Diskriminierung in der branchenspezifischen Segregation, d.h. in der Konzentration von Ausländern in einzelnen Branchen, besteht. 220 Um das Ausmass der branchenspezifischen Segregation zwischen In- und Ausländern vergleichen zu können, ist es notwendig, die Verteilung der Beschäftigten auf die verschiedenen Branchen in einer Masszahl zum Ausdruck zu bringen. In der Literatur werden zur Analyse der Arbeitsmarktsegmentation vorwiegend zwei Masse verwendet. ${ }^{221}$ Es handelt sich dabei einerseits um den sog. 'Dissimilarity-Index' $\boldsymbol{D}$, der auf DuNCAN/DunCAN (1955) zurückgeht, und andererseits um den von BUTLER (1987) eingeführten und von SILBER (1989a, 1989b) weiterentwickelten sog. 'G-Segregation-Index' $\boldsymbol{G}_{\boldsymbol{s}}$.

\subsubsection{Unterteilung der Branchen}

Zur empirischen Analyse der Arbeitsmarktsegmentation wird auf die sog. Allgemeine Nomenklatur der Wirtschaftszweige (ASWZ 85) des Bundesamtes für Statistik zurückgegriffen. ${ }^{222}$ In TAB. A9.17 in Anhang 1 findet sich eine Unterteilung der verschiedenen Wirtschaftszweige. Aus dieser Tabelle geht hervor, dass auf der höchsten Aggregationsebene neun Branchen unterschieden werden (siehe TAB. 9.5 und 9.6). In der amtlichen Statistik werden die einzelnen Wirtschaftszweige bis auf die 'Vier-Steller-Ebene' ausgewiesen. Um einerseits eine differenzierte Analyse der branchenspezifischen Segregation zu gewährleisten und andererseits eine genügende Zahl an Beobachtungen in den einzelnen Branchen sicherzustellen, erfolgt die Analyse der Arbeitsmarktsegmentation unter Verwendung der 'Zwei-Steller-Definition', wie sie in TAB. A9.17 ausgewiesen ist.

Die Zuordnung der befragten Personen nach Wirtschaftszweigen erfolgt im Rahmen der SAKE automatisch. Die befragten Personen müssen lediglich die Adresse des Betriebes angeben, in dem sie tätig sind. Mit Hilfe des Betriebs- und Unternehmensregisters findet dann automatisch die richtige Zuordnung der Wirtschaftsbranche statt. In denjenigen Fällen, in denen dies nicht möglich ist, wurden

220 In der Literatur finden sich dabei in erster Linie Analysen zur geschlechtsspezifischen Arbeitsmarktsegmentation. Ein Überblick findet sich in TAUBMANN/WACHTER (1986).

221 Siehe z.B. BoISSO/HAYES/HIRSCHBERG/SILBER (1994), DEUTSCH/FLÜCKIGER/SLBER (1994), FLÜCKIGER/BOYMOND/SILBER (1995) und SLLER (1989a, 1989b).

222 Seit 1995 wird vom Bundesamt für Statistik eine neue Definition der Wirtschaftszweige verwendet, die sog. Allgemeine Systematik der Wirtschaftszweige 1995 (NOGA). 
die befragten Personen aufgrund ihrer Selbsteinstufung zugeordnet, wobei in diesen Fällen lediglich eine Zuordnung auf dem 'Ein-Steller-Niveau' möglich war. ${ }^{223}$ Diese Vorgehensweise wurde deshalb gewählt, weil die Erfahrungen der Volkszählung gezeigt haben, dass die Befragten oft Mühe bekunden, die Wirtschaftsbranche ihres Betriebes richtig anzugeben.

\subsubsection{Segregationsmasse}

In der Literatur werden i.d.R. zwei Masse zur Analyse der Arbeitsmarktsegmentation verwendet. Es handelt sich dabei einerseits um den weitverbreiteten 'Dissimilarity-Index' sowie um den 'G-Segregation Index'. Aufgrund der weitgehenden Übereinstimmung zwischen diesen beiden Indizes, beziehen sich die nachfolgenden Ausführungen auf den 'Dissimilarity-Index'. Beide Indizes lehnen sich in ihrer Definition an die Literatur zur Einkommensverteilung an. DUNCAN/DUNCAN (1955) waren die ersten, welche die Erkenntnisse dieser Literatur zur Analyse der Arbeitsmarktsegmentation nutzten, indem sie zur Ableitung ihres Konzeptes der Segregationskurve auf die traditionelle Lorenzkurve der Einkommensverteilungsanalyse abstellten.224 Der von ihnen abgeleitete Dissimilarity-Index $\boldsymbol{D}$ ist wie folgt definiert:

$$
\mathrm{D}=0.5 \sum_{\mathrm{i}=1}^{\mathrm{n}}\left|\frac{\mathrm{F}_{\mathrm{i}}}{\mathrm{F}}-\frac{\mathrm{N}_{\mathrm{i}}}{\mathrm{N}}\right|,
$$

wobei $\mathrm{F}_{\mathrm{i}}$ und $\mathrm{N}_{\mathrm{i}}$ für die Zahl der aus- und inländischen Arbeitnehmer in Branche $\mathrm{i}$ stehen und $\mathrm{F}$ und $\mathrm{N}$ den Gesamtbestand an aus- und inländischen Arbeitnehmern repräsentieren. Wird aus den jeweiligen Verteilungen für die verschiedenen Branchen eine 'Lorenz'-artige Segregationskurve konstruiert, dann kann der Index D als grösste vertikale Distanz zwischen dieser Kurve und der Diagonalen verstanden werden. Anschaulicher lässt sich dieser Index als Anteil der Schweizer bzw. Ausländer interpretieren, der den Arbeitsplatz bzw. die Branche wechseln müsste, um eine Übereinstimmung in den Beschäftigungsanteilen in den verschiedenen Branchen zwischen In- und Ausländern zu gewährleisten. Dem Index D kommt in der

223 Die Verwendung des Betriebs- und Unternehmensregisters erlaubte in der SAKE des Jahres 1995 in 73 Prozent der Fälle eine automatische Bestimmung der Wirtschaftsbranche. Unter Berücksichtigung der Nachkodierungen des Bundesamtes für Statistik konnte in 92 Prozent aller Fälle eine Branchenzuteilung aufgrund der Betriebsadresse durchgeführt werden. Die verbleibenden 8 Prozent wurden aufgrund von Selbsteinstufungen zugeordnet [siehe BFS (1996b)].

224 Die Lorenzkurve bildet den sog. Gini-Koeffizienten als Konzentrationsmass für die Gleichbzw. Ungleichverteilung von Merkmalsträgern, z.B. der personellen Einkommensverteilung, ab. Der Gini-Koeffizient entspricht, bezogen auf die Lorenzkurve, dem Verhältnis der Verteilungsfläche zwischen der Diagonalen und der Lorenzkurve zur Fläche des Dreiecks unter der Diagonalen, die absolute Gleichverteilung und damit einen Gini-Koeffizienten von null impliziert. 
Literatur eine wichtige Rolle zu, da er für eine Reihe unterschiedlicher Segregationsfragestellungen verwendet werden kann. 225

\subsubsection{EMPIRISCHE ERGEBNISSE}

Wie bereits im Theorieteil ausgeführt wurde, erfordert eine umfassende Evaluation der Beschäftigungsperformance der Einwanderer neben der Analyse der Erwerbslosigkeit auch die Berücksichtigung der Arbeitsmarktsegmentation. Sie bildet einen komplementären Untersuchungsgegenstand zu den Probit-Schätzungen in Abschnitt 9.2.2. Zur Analyse der Arbeitsmarktsegmentation wird die Stichprobe der SAKE auf die ständige Wohnbevölkerung der Schweiz hochgerechnet, um eine bessere Vergleichbarkeit zu den Ergebnissen der deskriptiven Analyse zu ermöglichen. Gleichzeitig werden im Rahmen der Analyse der Arbeitsmarktsegmentation lediglich Vollzeiterwerbstätige betrachtet, da davon auszugehen ist, dass der Beschäftigungsgrad einen Einfluss auf die sektoralen Beschäftigungsanteile besitzt. Wie aus TAB. 9.18 hervorgeht, wurde der Dissimilarity-Index nicht nur für das Jahr 1995, sondern auch für das Jahr 1991 berechnet. Diese Vorgehensweise ermöglicht es, intertemporale Veränderungen im Ausmass der Arbeitsmarktsegmentation zumindest näherungsweise zu berücksichtigen.

Die Ergebnisse in TAB. 9.18 lassen sich wie folgt interpretieren. Ein Wert von null impliziert identische Beschäftigungsanteile der In- und Ausländer in den verschiedenen Branchen. Ein positiver Wert bedeutet demgegenüber eine Divergenz der in- und ausländischen Beschäftigungsanteile in den verschiedenen Wirtschaftszweigen. Je näher der Index bei eins liegt, desto grösser sind die Diskrepanzen in den Beschäftigungsanteilen zwischen In- und Ausländern. Bei einem Wert von eins besteht dementsprechend eine vollständige branchenspezifische Segregation zwischen In- und Ausländern. Wie bereits erwähnt wurde, dienen die inländischen

225 Eine alternative Messgrösse zur Berechnung der Arbeitsmarktsegmentation bildet, wie bereits erwähnt, der G-Segregation Index. Der Vorteil dieser Messgrösse besteht darin, dass er der relativen Bedeutung der verschiedenen Branchen an der Gesamtbeschäftigung Rechnung trägt [siehe auch FLÜCKIGER/BOYMOND/S匹BER (1995)]. Der G-Segregation Index entspricht dem gewichteten Gini-Index des Ausländer-/Inländerverhältnisses $\mathrm{F}_{\mathrm{i}} / \mathrm{N}_{\mathrm{i}}$, so dass

$$
G_{S}=0.5 \sum_{i=1}^{n} \sum_{j=1}^{n} \frac{N_{i}}{N} \cdot \frac{N_{j}}{N}\left|\frac{F_{i} / N_{i}-F_{j} / N_{j}}{F / N}\right| \text {. }
$$

Zur Vereinfachung der Berechnung lässt sich diese Gleichung, SLBER (1989a, 1989b) folgend, durch einige Umformungen wie folgt in Matrixschreibweise darstellen:

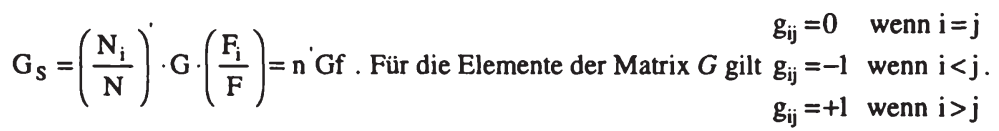

Siehe auch BOISSO/HAYES/HIRSCHBERG/SILBER (1994). 
Erwerbstätigen bzw. deren Beschäftigungsanteile als Referenzgrösse zur Berechnung des Dissimilarity-Index.

Die Ergebnisse zum Dissimilarity-Index in TAB. 9.18 zeigen deutlich, dass nicht nur zwischen Männern und Frauen, sondern auch zwischen den einzelnen Nationalitätengruppen signifikante Unterschiede im Ausmass der branchenspezifischen Segregation bestehen. Im einzelnen stellen sich die Ergebnisse wie folgt dar. Erstens hat zwischen 1991 und 1995 vor allem bei den Männern eine Anpassung der ausländischen an die inländische Beschäftigungsstruktur stattgefunden. ${ }^{226}$ Zweitens ist klar erkennbar, dass bei den Männern grössere Unterschiede zwischen den In- und Ausländern bestehen als bei den Frauen. Dieses Ergebnis hängt mit der grösseren Streuung über die verschiedenen Branchen bei den Männern zusammen. Drittens bestehen auch zwischen den verschiedenen Nationalitätengruppen substantielle Diskrepanzen im Ausmass der branchenspezifischen Segregation. Dies trifft insbesondere für die Einwanderer aus nicht-europäischen Staaten zu. Wie aus Tab. A9.18 in Anhang 1 hervorgeht, bestehen die geringsten Unterschiede bei beiden Geschlechtern für Einwanderer aus Italien, Spanien, Deutschland und Frankreich.

Tab. 9.18: Dissimilarity-Index D: Ergebnisse zur branchenspezifischen Segregation

\begin{tabular}{|c|c|c|c|c|c|c|}
\hline \multirow{3}{*}{$\begin{array}{l}\text { Nationalitäten- } \\
\text { gruppen }\end{array}$} & \multicolumn{6}{|c|}{ DISSIMILARITY-INDEX D } \\
\hline & \multicolumn{2}{|c|}{ Frauen } & \multicolumn{2}{|c|}{ Männer } & \multicolumn{2}{|c|}{ Total } \\
\hline & 1991 & 1995 & 1991 & 1995 & 1991 & 1995 \\
\hline Nordeuropa & 0.264 & 0.278 & 0.307 & 0.301 & 0.234 & 0.248 \\
\hline Südeuropa & 0.290 & 0.281 & 0.370 & 0.345 & 0.319 & 0.285 \\
\hline Nicht-Europa & 0.468 & 0.428 & 0.382 & 0.395 & 0.354 & 0.365 \\
\hline
\end{tabular}

Quelle: SAKE (1995), eigene Berechnungen

Anmerkungen: Zur Aufschlüsselung der Nationalitätengruppen siehe TAB. 8.2

Aus TAB. 9.18 lassen sich zwar Aussagen über das Ausmass der Arbeitsmarktsegmentation bzw. der branchenspezifischen Segregation ableiten, nicht jedoch darüber, welche Branchen für die Segregation verantwortlich sind. Es ist also nicht klar, welche Branchen für die divergierenden Beschäftigungsanteile verantwortlich sind. Dieser Punkt ist jedoch für die Evaluation der Beschäftigungsperformance von zentraler Bedeutung, da eine stärkere Beschäftigungskonzentration der Ausländer in Branchen die ein hohes (niedriges) Qualifikationsprofil erfordern nicht nur eine Zunahme der branchenspezifischen Segregation impliziert, sondern gleichzeitig auch eine Verbesserung (Verschlechterung) der Beschäftigungsperformance der Ausländer zur Folge hat.

226 Es ist jedoch darauf hinzuweisen, dass im Rahmen dieses Assimilierungsprozesses sowohl ein 'upgrading' als auch ein 'downgrading' der Beschäftigungsstruktur der ausländischen Erwerbstätigen stattfinden kann. Dieser Aspekt wird im folgenden ausführlicher diskutiert. 
Dieser Punkt lässt sich jedoch unter Berücksichtigung der Ergebnisse in TAB. 9.5 klären, in der die Beschäftigungsanteile der verschiedenen Nationalitätengruppen an den einzelnen Wirtschaftszweigen auf dem Ein-Steller-Niveau ausgewiesen sind. Während die Einwanderer aus Süd- und Nicht-Europa, mit Ausnahme der Ausländer aus den aussereuropäischen Staaten, in weit stärkerem Mass im sekundären Sektor konzentriert sind als die Inländer, weisen die Ausländer aus Nordeuropa eine stärkere Beschäftigungskonzentration im Dienstleistungssektor auf, insbesondere im Banken-/Versicherungs- und Immobilien-Sektor, bei den sonstigen Dienstleistungen sowie im Handel und Gastgewerbe. Demgegenüber besitzen die inländischen Erwerbstätigen im primären Sektor sowie im Bereich der öffentlichen Verwaltung höhere Beschäftigungsanteile als die Ausländer, was mit den gesetzlichen Bestimmungen zur Ausländererwerbstätigkeit zusammenhängt.

Zusammenfassend ergibt sich somit die Schlussfolgerung, dass für die Einwanderer aus Nordeuropa mit einem positiven und für die Einwanderer aus Süd- und Nicht-Europa mit einem negativen Effekt der branchenspezifischen Segregation auf die Beschäftigungsperformance zu rechnen ist. In Übereinstimmung mit den Überlegungen zur dualen Arbeitsmarkttheorie impliziert die Segmentation des Arbeitsmarktes, dass Süd- und Nicht-Europäer vorwiegend in denjenigen Branchen tätig sind, die für Inländer und Nordeuropäer wenig attraktiv sind und für die zu vermuten ist, dass sie durch instabilere Beschäftigungsverhältnisse sowie tiefere Einkommen charakterisiert sind.

\subsection{FAZIT}

Aus den Ausführungen zur Beschäftigungsperformance dieses Kapitels lassen sich drei wesentliche Schlussfolgerungen ableiten. Erstens haben die Ergebnisse der deskriptiven Analyse gezeigt, dass substantielle geschlechts-, kohorten- und nationalitätenspezifische Unterschiede in den invididuellen Charakteristika sowie damit verbunden in der Beschäftigungsperformance bestehen. Diese Divergenzen bestehen sowohl hinsichtlich der beruflichen Stellung und der Beschäftigung nach Wirtschaftszweigen als auch hinsichtlich der Erwerbstätigkeit und der Erwerbslosigkeit.

Zweitens hat die empirische Analyse der Erwerbslosigkeit gezeigt, dass Ausländer im Mittel eine vergleichsweise höhere Erwerbslosigkeitswahrscheinlichkeit aufweisen als Schweizer. Dieses Ergebnis ist auf Unterschiede in den individuellen Charakteristika zwischen In- und Ausländern zurückzuführen. Es zeigt sich aber auch, dass zwischen den drei Nationalitätengruppen signifikante Unterschiede in den Erwerbslosigkeitswahrscheinlichkeiten bestehen. Sowohl bei den Männern als auch bei den Frauen liegen diese für die Zuwanderer aus Nordeuropa deutlich unter jenen der Süd- und Nicht-Europäer.

Drittens haben die Ausführungen zur Arbeitsmarktsegmentation gezeigt, dass zwischen den In- und Ausländern bzw. den verschiedenen Nationalitätengruppen 
klare Unterschiede in der Branchenzugehörigkeit ihrer Arbeitskräfte bestehen. Die Analyse der Arbeitsmarktsegmentation liefert damit einige Anhaltspunkte dafür, dass von der Segmentation für die Zuwanderer aus Nordeuropa ein positiver und für die Zuwanderer aus Süd- und Nicht-Europa ein negativer Effekt auf die Beschäftigungsperformance ausgeht. 


\section{EMPIRISCHE ANALYSE DER EINKOMMENSPERFORMANCE}

Neben der Beschäftigungsperformance, die im Zentrum des vorangegangenen Kapitels stand, bildet die Einkommensperformance den zweiten wichtigen Ansatz zur Analyse der Arbeitsmarktperformance. Wie bereits mehrfach erwähnt wurde, handelt es sich bei der Beschäftigungs- und der Einkommensperformance um zwei komplementäre und interdependente Ansätze. Es ist jedoch davon auszugehen, dass der Zusammenhang zwischen diesen beiden Ansätzen in erster Linie von der Beschäftigungsperformance bestimmt wird. Diese Überlegungen lassen sich anhand der TAB. 9.5 - 9.8 illustrieren, in denen die berufliche Stellung sowie die Beschäftigung der in- und ausländischen Wirtschaftssubjekte nach Wirtschaftszweigen abgebildet ist. Eine Verbesserung der Beschäftigungsperformance, die sich sowohl in einem vertikalen Aufstieg hinsichtlich der beruflichen Stellung als auch in einem horizontalen Wechsel in einen vorteilhafteren Wirtschaftszweig äussern kann, ist i.d.R. auch mit einer Verbesserung der Einkommensperformance verbunden.

Analog zum vorangegangenen Kapitel werden auch in diesem Kapitel sowohl produktivitätsbezogene als auch diskriminierungsbedingte Faktoren bei der Analyse der Einkommensperformance berücksichtigt. Die deskriptive Analyse in Kapitel 3 hat gezeigt, dass zwischen In- und Ausländern substantielle Unterschiede in den Einkommensniveaus bestehen. Diese lassen sich, wie bereits angedeutet, vor allem auf Divergenzen in der Humankapitalausstattung, der beruflichen Stellung sowie der Beschäftigung nach Wirtschaftszweigen zurückführen. In diesem Kapitel werden diese Zusammenhänge ausführlich mit Hilfe ökonometrischer Verfahren sowie deskriptiver Statistiken erörtert. Das Ziel dieses Kapitels besteht einerseits darin zu analysieren, ob zwischen In- und Ausländern signifikante Unterschiede in den Einkommen bestehen, sowie andererseits zu untersuchen, worauf diese Unterschiede zurückzuführen sind.

Die Vorgehensweise gestaltet sich analog zu jener des vorangegangenen Kapitels. In einem ersten Schritt werden einige deskriptive Statistiken präsentiert. In einem zweiten Schritt folgt die Diskussion der Ergebnisse der produktivitätsbedingten Determinanten der Einkommensperformance. In einem dritten Schritt werden dann die diskriminierungsbedingten Einflussfaktoren erörtert. In einem vierten Schritt wird schliesslich ein kurzes Fazit gezogen. 


\subsection{DESKRIPTIVE ANALYSE DER EINKOMMENSPERFORMANCE}

Analog zum vorangegangenen Kapitel lassen sich aus der deskriptiven Analyse bereits erste Anhaltspunkte auf Performanceunterschiede zwischen In- und Ausländern sowie zwischen den verschiedenen Nationalitätengruppen gewinnen.

\subsubsection{EINKOMMENSHÖHE NACH NATIONALITÄTENGRUPPEN}

In TAB. 10.1 ist die Einkommenshöhe für Männer und Frauen nach Nationalitätengruppen abgebildet. ${ }^{227}$ Es zeigt sich erwartungsgemäss, dass sowohl zwischen den In- und Ausländern der verschiedenen Nationalitätengruppen als auch zwischen Männern und Frauen beträchtliche Einkommensunterschiede bestehen. Mit Blick auf die männlichen Erwerbstätigen ist ersichtlich, dass der Anteil der Ausländer in den Einkommenssegmenten bis $65^{\prime} 000$ CHF über jenem der Inländer liegt. Für die höheren Einkommenssegmente trifft demgegenüber genau das $\mathrm{Ge}-$ genteil zu. Die Berücksichtigung der verschiedenen Nationalitätengruppen zeigt, dass der hohe Ausländeranteil in den tiefen Einkommenssegmenten vor allem durch die Zuwanderer aus nicht-europäischen Staaten bedingt ist. Die Einwanderer aus dem süd-europäischen Raum sind demgegenüber stärker in den mittleren Einkommenssegmenten vertreten. Hinsichtlich der Zuwanderer aus nordeuropäischen Staaten ist schliesslich erkennbar, dass diese nicht nur ein deutlich vorteilhafteres Einkommensprofil besitzen als die Ausländer der anderen Nationalitätengruppen, sondern auch als die Einheimischen. Im höchsten Einkommenssegment ist der Anteil der Nordeuropäer sowohl bei den Männern als auch bei den Frauen rund doppelt so hoch wie bei den Inländern.

Diese Ergebnisse stehen in Einklang mit den theoretischen Überlegungen in Kapitel 5 sowie den empirischen Ergebnissen in Kapitel 9. Einerseits hat der Theorieteil gezeigt, dass mit zunehmender Aufenthaltsdauer eine Assimilierung der Einkommen der Ausländer an jene der Inländer zu erwarten ist. Diese Hypothese scheint sich mit Blick auf die Einkommensunterschiede zwischen den Einwanderern aus Südeuropa und jenen aus Nicht-Europa zu bestätigen, da letztere im Mittel eine signifikant kürzere Aufenthaltsdauer aufweisen (siehe Abschnitt 8.3). Andererseits lässt sich die deutlich bessere Performance der Nordeuropäer im Vergleich zu den Süd- und Nicht-Europäern, auf die bessere Transferierbarkeit des Humankapitals sowie auf die relativ bessere Selektion zurückführen. Diese Überlegungen werden mit Blick auf die empirischen Ergebnisse zur Beschäftigungsperformance bestätigt, denen zufolge die Nordeuropäer im Vergleich zu den Süd- und Nicht-Europäern nicht nur vorteilhaftere sozio-ökonomische Profile, sondern auch signifikant geringere Erwerbslosigkeitswahrscheinlichkeiten aufweisen.

227 Die Ergebnisse für die detaillierte Aufgliederung der Nationalitätengruppen findet sich in TAB. A10.1 in Anhang 1. 
Tab. 10.1: Einkommenshöhe der erwerbstätigen Bevölkerung nach Nationalitätengruppen (in Prozent)

\begin{tabular}{|c|c|c|c|c|c|c|c|}
\hline \multirow{2}{*}{$\begin{array}{l}\text { Nationalitäten- } \\
\text { gruppen }\end{array}$} & \multicolumn{7}{|c|}{ Einkommenshöhe } \\
\hline & 1 & 2 & 3 & 4 & 5 & 6 & 7 \\
\hline \multicolumn{8}{|l|}{ Männer } \\
\hline Nordeuropa & 1.6 & 0.6 & 5.1 & 18.0 & 18.4 & 36.4 & 19.9 \\
\hline Südeuropa & 0.6 & 4.5 & 17.3 & 43.0 & 24.7 & 8.1 & 1.8 \\
\hline Nicht-Europa & 1.9 & 14.8 & 23.6 & 30.9 & 14.4 & 12.6 & 1.8 \\
\hline Ausländer & 1.2 & 5.4 & 14.9 & 33.1 & 20.7 & 17.5 & 7.2 \\
\hline Schweizer & 2.4 & 2.1 & 9.8 & 19.0 & 21.8 & 35.9 & 9.1 \\
\hline \multicolumn{8}{|l|}{ Frauen } \\
\hline Nordeuropa & 2.3 & 7.5 & 15.5 & 25.2 & 24.5 & 22.6 & 2.4 \\
\hline Südeuropa & 7.4 & 33.2 & 40.5 & 15.1 & 3.0 & 0.9 & \\
\hline Nicht-Europa & 9.3 & 27.7 & 33.2 & 12.5 & 14.2 & 3.2 & \\
\hline Ausländer & 6.2 & 24.4 & 31.5 & 17.6 & 11.7 & 7.9 & 0.7 \\
\hline Schweizer & 6.0 & 11.3 & 27.2 & 23.9 & 15.0 & 15.1 & 1.4 \\
\hline
\end{tabular}

Quelle: SAKE (1995), eigene Berechnungen

Anmerkungen: Erwerbstätige Bevölkerung: Personen zwischen 16 und 64 Jahren

Zur Aufschlüsselung der Nationalitätengruppen siehe TAB. 8.2

Einkommenshöhe in CHF: $1=<26^{\prime} 000 \mathrm{CHF}, 2=26^{\prime} 001-39^{\prime} 000 \mathrm{CHF}, 3=39^{\prime} 001-$ $52^{\prime} 000 \mathrm{CHF}, 4=52^{\prime} 001-65^{\prime} 000 \mathrm{CHF}, 5=65^{\prime} 001-78^{\prime} 000 \mathrm{CHF}, 6=78^{\prime} 001-130^{\prime} 000$ $\mathrm{CHF}, 7=>130^{\prime} 000 \mathrm{CHF}$

In TAB. 10.1 sind ebenfalls geschlechtsspezifische Unterschiede in den Einkommensprofilen erkennbar. Am auffälligsten ist die deutlich stärkere Konzentration der weiblichen Erwerbstätigen in den tieferen Einkommenssegmenten. Während rund 25 Prozent der Ausländer und 45 Prozent der Inländer ein Erwerbseinkommen von mehr als 78'000 CHF aufweisen, liegt der Anteil bei den Ausländerinnen bei lediglich rund 8.5 Prozent und bei den Inländerinnen bei 16.5 Prozent. Diese substantiellen geschlechtsspezifischen Unterschiede lassen sich auf verschiedene Ursachen zurückführen.

Erstens hat die Analyse in Kapitel 9 gezeigt, dass der Anteil der Frauen in den höchsten Ausbildungssegmenten z.T. beträchtlich unter jenem der Männer liegt, so dass sich die geschlechtsspezifischen Unterschiede in den Einkommen zumindest teilweise auf divergierende Humankapitalausstattungen zurückführen lassen. Zweitens haben die deskriptiven Statistiken des vorangegangenen Kapitels gezeigt, dass die weiblichen Erwerbstätigen stärker in tieferen Hierarchiestufen vertreten sind als die männlichen Erwerbstätigen. Drittens besitzen auch die divergierenden Beschäftigungsanteile der Männer und Frauen in den verschiedenen Wirtschaftszweigen einen Einfluss auf die Einkommenshöhe. Viertens ist schliesslich 
nicht auszuschliessen, dass diese Unterschiede durch Einkommensdiskriminierung bedingt sind. ${ }^{228}$

\subsubsection{EINKOMMENSHÖHE NACH EINWANDERUNGSKOHORTEN}

Bei der Interpretation der Ergebnisse in TAB. 10.1 gilt es allerdings zu beachten, dass die Differenzierung der Analyse nur nach Nationalitätengruppen erfolgte, nicht aber hinsichtlich der Zuzugsjahre bzw. der Aufenthaltsdauer. Die Relevanz des Einwanderungszeitpunktes bzw. der Aufenthaltsdauer ist jedoch mit Blick auf die Ausführungen zur Einkommensassimilierung in Kapitel 5 evident. Dem bedeutenden Beitrag von CHISWICK (1978) folgend, ist davon auszugehen, dass Einwanderer aufgrund der Abwertung ihres Humankapitals (insbesondere der Sprachkenntnisse) unmittelbar nach der Einwanderung weniger verdienen als vergleichbare Einheimische. Aufgrund des hohen Ertragspotentials investieren die Einwanderer aber i.d.R. verstärkt in ziellandspezifisches Humankapital, so dass im Zeitverlauf eine Angleichung der Einkommen der Ausländer an jene der Inländer stattfindet. CHISWICK kommt im Rahmen seiner empirischen Analyse zum Ergebnis, dass einige Einwanderergruppen aufgrund der positiven Selektion letztlich sogar mehr verdienen als die Einheimischen.

Erste Hinweise auf diese Zusammenhänge vermag die Berücksichtigung der Aufenthaltsdauer im Rahmen der deskriptiven Analyse in TAB. $10.2 \mathrm{zu}$ geben. Augenfällig ist sowohl bei den Frauen als auch bei den Männern der hohe Anteil an $\mathrm{Zu}$ wanderern der jüngsten Einwanderungskohorte in den obersten Einkommenssegmenten. Dieses Ergebnis, das im Widerspruch zur oben beschriebenen Assimilierungshypothese steht, lässt sich u.a. auf die veränderten Rahmenbedingungen in der Ausländerpolitik zurückführen, die in jüngster Zeit verstärkt auf die Zuwanderung höherqualifizierter Ausländer ausgerichtet ist. Diese Überlegung wird durch die Ergebnisse in TAB. 8.6 gestützt, aus der hervorgeht, dass die Nordeuropäer in den jüngsten Einwanderungskohorten stark vertreten sind. Aus TAB. 10.2 ist schliesslich ersichtlich, dass zumindest eine partielle Einkommensassimlierung der Einwanderer stattgefunden hat, da sich mit zunehmender Aufenthaltsdauer eine Verlagerung von den untersten in die intermediären Einkommenssegmente beobachten lässt. Diese Überlegungen gilt es jedoch im Rahmen der empirischen Analyse zu überprüfen.

228 Wie KUGLER (1988) in einer Analyse der geschlechtsspezifischen Diskriminierung in der Schweiz zeigen konnte, lässt sich das Einkommensdifferential bei den Inländern, nicht aber bei den Ausländern, zum grössten Teil durch Ausstattungsunterschiede erklären. In einer Studie von DiEKMAN/ENGELHARDT (1994), in der lediglich inländische Männer und Frauen berücksichtigt werden, fällt der Diskriminierungseffekt allerdings deutlich höher aus. 
Tab. 10.2: Einkommenshöhe der erwerbstätigen Bevölkerung nach Einwanderungskohorten (in Prozent)

\begin{tabular}{|l|rrrrrrr|}
\hline $\begin{array}{l}\text { Einwande- } \\
\text { rungskohorten }\end{array}$ & \multicolumn{7}{|c|}{ Einkommenshöhe } \\
\hline Männer & & 2 & $\mathbf{3}$ & $\mathbf{4}$ & $\mathbf{5}$ & $\mathbf{6}$ & $\mathbf{7}$ \\
$<1$ & 8.3 & & 7.5 & 15.0 & 15.5 & 13.0 & 40.8 \\
$1-5$ & 1.4 & 12.6 & 16.8 & 18.7 & 17.8 & 20.7 & 12.1 \\
$5-10$ & 0.2 & 8.4 & 20.6 & 37.3 & 10.9 & 15.0 & 7.6 \\
$10-15$ & 1.2 & 5.5 & 23.1 & 37.9 & 11.5 & 16.6 & 4.1 \\
$15-20$ & 2.9 & 3.2 & 13.4 & 31.1 & 31.0 & 15.2 & 3.2 \\
$>20$ & 0.9 & 3.4 & 8.7 & 32.8 & 27.8 & 19.2 & 7.3 \\
\hline Frauen & & & & & & & \\
$<1$ & & 41.7 & & & & 58.3 & \\
$1-5$ & 5.9 & 35.6 & 17.9 & 17.9 & 10.0 & 8.9 & 3.8 \\
$5-10$ & 11.6 & 23.7 & 37.7 & 9.0 & 15.7 & 2.3 & \\
$10-15$ & 0.9 & 17.5 & 30.3 & 21.3 & 21.4 & 8.7 & \\
$15-20$ & 10.0 & 33.4 & 10.6 & 26.9 & 9.8 & 9.3 & \\
$>20$ & 4.6 & 16.9 & 44.7 & 18.8 & 6.8 & 8.2 & \\
\hline
\end{tabular}

Quelle: SAKE (1995), eigene Berechnungen

Anmerkungen: Erwerbstätige Bevölkerung: Personen zwischen 16 und 64 Jahren

Einwanderungskohorten: Aufenthaltsdauer in Jahren

Einkommenshöhe in CHF: $1=\left\langle 26^{\prime} 000 \mathrm{CHF}, 2=26^{\prime} 001-39^{\prime} 000 \mathrm{CHF}, 3=39^{\prime} 001-\right.$ $52^{\prime} 000 \mathrm{CHF}, 4=52^{\prime} 001-65^{\prime} 000 \mathrm{CHF}, 5=65^{\prime} 001-78^{\prime} 000 \mathrm{CHF}, 6=78^{\prime} 001-130^{\prime} 000$ $\mathrm{CHF}, 7=>130^{\prime} 000 \mathrm{CHF}$

\subsection{EMPIRISCHE ANALYSE DER EINKOMMEN}

Die Analyse der Einkommensperformance umfasst zwei wichtige, interdependente Ansätze, die in diesem sowie im nachfolgenden Abschnitt erörtert werden. Zum einen gilt es zu untersuchen, ob zwischen In- und Ausländern signifikante Unterschiede in den Einkommensprofilen bestehen. Zur Analyse dieser Fragestellung werden Einkommensfunktionen, die auf der Humankapitaltheorie basieren, unter Verwendung der OLS-Methode geschätzt. Von Interesse ist in diesem Zusammenhang vor allem die Untersuchung der intertemporalen Entwicklung der Einkommen, d.h. die Einkommensassimilierung. Zum anderen muss analysiert werden, ob Einkommensunterschiede zwischen In- und Ausländer auf produktivitätsbezogene oder diskriminierungsbedingte Faktoren zurückzuführen sind. Zur Untersuchung der Einkommensdiskriminierung wird der von OAXACA (1973) und BLINDER (1973) entwickelte Ansatz der Einkommenszerlegung (earnings difference decomposition approach) verwendet. 


\subsubsection{METHODISCHE VORGEHENSWEISE}

Als Ausgangspunkt für die Analyse der Einkommensperformance dient i.d.R. die von MINCER (1974) entwickelte Einkommensfunktion, in der das (logarithmierte) Einkommen linear mit einer Reihe erklärender Variablen in Beziehung gesetzt wird. Im Gegensatz zur ökonometrischen Analyse in Kapitel 9, gestaltet sich die Untersuchung der Einkommensperformance vergleichsweise einfach, da es sich bei der zu erklärenden Variablen, dem Einkommensniveau, nicht um eine dichotome, qualitative, sondern um eine stetige, quantitative Variable handelt. Aus diesem Grund kann zur Schätzung der Einkommmensfunktionen auf das traditionelle ökonometrische Instrumentarium, d.h. die OLS-Schätzmethode, zurückgegriffen werden. Ein Problem, das es bei der Analyse von Einkommensfunktionen allerdings zu berücksichtigen gilt, besteht im sog. Stichproben-Auswahlfehler (sample-selection-bias), der auf der Überlegung beruht, dass die zur Schätzung der Einkommensfunktion verwendete Stichprobe nicht notwendigerweise auf einer stochastischen Auswahl aus der Gesamtbevölkerung beruht. Dieser Selektivitäts-Bias führt i.d.R. zu Verzerrungen der geschätzten Regressionskoeffizienten der Einkommensfunktion. Dieser Verzerrung kann mit dem von HECKMAN (1976a) vorgeschlagenen zweistufigen Schätzverfahren Rechnung getragen werden, das in Abschnitt 10.2.1.3 ausführlich diskutiert wird.

\subsubsection{Modellspezifikation}

Der empirischen Analyse dieses Kapitels liegt, wie bereits erwähnt, das Humankapitalmodell zugrunde, dem in der empirischen Wirtschaftsforschung grosse Bedeutung zukommt. ${ }^{229}$ Das Ziel dieses Abschnitts besteht in der Modellspezifikation, d.h. in der Formulierung der funktionalen Form der Regressionsgleichung. Studien zur Lohn- und Einkommensverteilung haben gezeigt, dass Lohnund Einkommensdaten typischerweise eine rechtsschiefe Verteilung aufweisen. Dies impliziert, dass das Medianeinkommen links vom Durchschnittseinkommen liegt. Um dieser Verteilungsform Rechnung zu tragen, wird in der Literatur häufig auf die sog. Lognormalverteilung zurückgegriffen, welche eine relativ gute Ab-

229 Auch wenn die Humankapitaltheorie bei der Analyse von Einkommensfunktionen weitverbreitete Anwendung findet, existieren verschiedene alternative Ansätze. In diesem Zusammenhang ist insbesondere auf die sog. 'screening'-Theorie zu verweisen, die bereits in Kapitel 6 diskutiert wurde. Dieser Ansatz geht auf ARROw (1973b) und SPENCE $(1973,1974)$ zurück. Die 'screening'-Theorie basiert im Gegensatz zur Humankapitaltheorie auf der Annahme, dass Aus- und Weiterbildung einen geringen Einfluss auf die Produktivität der Wirtschaftssubjekte besitzen. Unternehmen verwenden Qualifikationen jedoch als Signale bzw. Indikatoren zur Einschätzung der Produktivität und Fähigkeiten der Wirtschaftssubjekte. Die Ausbildung bzw. die Humankapitalausstattung dienen somit als Selektionsinstrument bei der Rekrutierung neuer Arbeitnehmer, wobei sie die Produktivität der einzelnen Wirtschaftssubjekte aber nicht direkt beeinflusst. 
bildung der effektiven Einkommensverteilung ermöglicht. ${ }^{230}$ Zudem gibt es Studien, die sich mit der Beziehung zwischen der Einkommensverteilung und den sie erklärenden Faktoren befassen. So konnte RoY (1950) zeigen, dass nicht das Einkommen sondern der Logarithmus des Einkommens normalverteilt ist, wenn die Bestimmungsfaktoren des Einkommens normalverteilt sind und wenn das Einkommen mit dem Produkt von zwei oder mehreren unkorrelierten Faktoren variiert.

Aus diesen Gründen wird für die empirische Analyse der Einkommensperformance der natürliche Logarithmus des monatlichen Bruttoerwerbseinkommens (LNINCGM) als endogene, zu erklärende Variable verwendet. Zwei Punkte gilt es dabei zu beachten. Erstens ergibt sich aufgrund der Freiwilligkeit der SAKE-Befragung das Problem, dass Antworten zu gewissen Fragen verweigert werden können. Wie bereits aus TAB. 3.9 hervorging, haben rund 20 Prozent der Befragten in der Gesamtstichprobe keine Angaben zum Einkommen gemacht. Diesen Umstand gilt es, bei der Interpretation der Ergebnisse zu berücksichtigen.

Zweitens besteht für diejenigen, die Angaben zum Einkommen machen die Möglichkeit, diese entweder auf Stunden-, Monats- oder Jahresbasis zu machen. Um Verzerrungen aufgrund von Umrechnungsfehlern zu minimieren, werden Monatsangaben verwendet, da diese Form der Einkommensangabe von rund 90 Prozent der Antwortenden gewählt wurde. Zur Vermeidung von Einkommensunterschieden, die auf divergierende Beschäftigungsgrade bzw. Wochenarbeitszeiten zurückzuführen sind, werden zur Analyse der Einkommensperformance lediglich Vollzeiterwerbstätige, d.h. Erwerbstätige mit einem Beschäftigungsgrad von 90100 Prozent berücksichtigt. Analog zur bisherigen Vorgehensweise werden darüber hinaus lediglich die 16- bis 64-jährigen Erwerbstätigen in der Analyse berücksichtigt. Schliesslich werden aufgrund der in Kapitel 2 angesprochenen arbeitsmarktlichen Zugangsbeschränkungen für Ausländer lediglich abhängige Erwerbstätige im privatwirtschaftlichen Sektor berücksichtigt.

Die ökonometrische Literatur zur Einkommensbestimmung basiert i.d.R. auf semi-logarithmischen Regressionsgleichungen der Form

(10.1) $\quad \ln y_{i}=f\left(x_{i}\right)+u_{i} \quad i=1, \ldots, n$

wobei $\ln y_{i}$ den natürlichen Logarithmus des Einkommens oder des Lohnes von Individuum $i$ bezeichnet, $x_{i}$ für eine Reihe exogener, erklärender Variablen des Einkommens steht und $\mathrm{u}_{\mathrm{i}}$ ein stochastischer Störterm ist, der die unbeobachtbaren Charakteristika sowie die inhärente Zufälligkeit der Einkommensstatistik widerspiegelt. Es wird i.a. unterstellt, dass $u_{i} \approx N\left(0, \sigma^{2}\right)$ verteilt ist. MINCER (1974) folgend, werden als zentrale erklärende Variablen die Schulausbildung (SCH) so-

230 Siehe BERNDT (1996). Mit der Lognormalverteilung lässt sich allerdings nicht das Auftreten hoher Einkommen erklären. 
wie die Arbeitserfahrung (EXPE) verwendet. ${ }^{231}$ Die Arbeitserfahrungsvariable EXPE berechnet sich wie folgt aus dem Alter, der Anzahl der Schuljahre sowie einer Konstanten, welche die erste Lebensphase vor dem Schuleintritt approximiert:

(10.2) $\quad \mathrm{EXPE}=\mathrm{AGE}-\mathrm{SCH}-6.5$.

Hinsichtlich der Ausbildungsvariablen ist von einem positiven Zusammenhang zur Einkommenshöhe auszugehen, da mit zunehmender Ausbildung die Ertragsrate auf Bildung steigt. Der Humankapitaltheorie folgend, wird davon ausgegangen, dass das Einkommen über den Lebenszyklus nicht konstant ist, sondern einen konkaven Verlauf aufweist, mit einem Wendepunkt in der Mitte des Erwerbslebens. ${ }^{232}$ Diese Überlegungen implizieren, dass die MINCER'SCHE Einkommensfunktion um den quadratischen Term $\boldsymbol{E X P S Q}$ erweitert werden sollte, um den abnehmenden Grenzertrag der Arbeitserfahrung berücksichtigen zu können. ${ }^{233}$ Es ist allerdings mit Blick auf die ausländischen Erwerbstätigen nicht auszuschliessen, dass sowohl die Ausbildung als auch die Arbeitserfahrung, aufgrund der unvollständigen Transferierbarkeit von Humankapital, einen vergleichsweise geringen bzw. nicht-signifikanten Einfluss auf das Einkommen besitzen.

Neben diesen personenspezifischen Charakteristika besitzen auch berufsspezifische Merkmale eine wichtige Rolle bei der Einkommensbestimmung. Um der beruflichen Stellung der betrachteten Personen Rechnung zu tragen, werden zwei Dummy-Variablen für Erwerbstätige mit Vorgesetztenfunktion (EMWM) sowie für Erwerbstätige mit Aufgaben in der Unternehmensleitung (EMBM) in der Ein-

231 Zur Konstruktion der Ausbildungsvariablen siehe Abschnitt 9.2.1.

232 Wie bereits in den Kapiteln 4 und 5 ausgeführt wurde, ist es für Wirtschaftssubjekte im allgemeinen optimal, in jungen Jahren in Humankapital zu investieren. In dem Ausmass, in dem Arbeitserfahrung als Investition in 'on-the-job-training' angesehen werden kann, verringern sich die Anreize für diese Form der Humankapitalakkumulation mit zunehmendem Alter. Der Hauptgrund hierfür besteht darin, dass der Gegenwartswert dieser Investitionen mit der Verkürzung der Erwerbsphase sinkt. Den Arbeiten von GHEZ/BECKER (1974) sowie BLINDER/WEISS (1976) folgend, ergibt sich die Schlussfolgerung, dass es aufgrund des sich verringernden Gegenwartswertes von Erträgen auf Investitionen i.a. optimal ist, die Arbeitszeit in der ersten Phase des Erwerbslebens zu erhöhen, um so innerhalb eines kurzen Zeitraums Humankapital durch Arbeitserfahrung zu akkumulieren. Um die Mitte des Erwerbslebens wird dann der Wendepunkt erreicht, der mit einer Verkürzung der Arbeitszeit verbunden sein sollte, da sich die Investitionsanreize verringern. Einen analogen Verlauf weist dementsprechend auch das Einkommensprofil auf, das sich aus dem Produkt des Lohnsatzes und der Jahresarbeitszeit zusammensetzt. HECKMAN (1976b), KILLINGSWORTH (1982) und WEISS (1986) haben in diesem Zusammenhang jedoch darauf hingewiesen, dass die Verläufe sowie die Wendepunkte dieser Einkommensprofile, in Abhängigkeit der spezifischen Art der Arbeitserfahrung sowie des Diskontfaktors, stark divergieren können.

233 Aufgrund der hohen Multikollinearität zwischen EXPE und EXPSQ musste allerdings auf eine Berücksichtigung des quadratischen Terms verzichtet werden. 
kommensfunktion berücksichtigt. Hinsichtlich der berufsspezifischen Variablen ist ebenfalls von einem positiven Zusammenhang zur Erklärung der Einkommenshöhe auszugehen.

Desweiteren ist anzunehmen, dass neben berufs- auch branchenspezifische Faktoren einen Einfluss auf die Einkommenshöhe besitzen. Diesem Umstand wird durch die Berücksichtigung einer Dummy-Variablen für Branchen im tertiären Sektor (SECTOR3) Rechnung getragen, die ein höheres Qualifikationsprofil erfordern. Es handelt sich dabei um die Wirtschaftszweige 6-8 in TAB. 9.5, d.h. Verkehr/Nachrichten, Banken/Immobilien/Versicherungen sowie sonstige Dienstleistungen. ${ }^{234}$ Auch hier ist davon auszugehen, dass die Beschäftigung in den höherqualifizierten Segmenten des tertiären Sektors einen positiven Effekt auf das Einkommen besitzt.

Neben diesen mikroökonomischen Variablen wird mit den kantonalen Arbeitslosenquoten (UNPL) schliesslich auch ein makroökonomischer Indikator berücksichtigt, der den Einfluss der wirtschaftlichen Lage auf die Einkommenshöhe misst. Es ist davon auszugehen, dass ungünstige wirtschaftliche Rahmenbedingungen eine dämpfende Wirkung auf die Lohnforderungen der Arbeitnehmer und Gewerkschaften haben werden, so dass mit einem negativen Zusammenhang zwischen der Höhe der Arbeitslosenquote und dem Einkommensniveau zu rechnen ist.

Mit Blick auf die Einkommensperformance der Einwanderer gilt es schliesslich einen wichtigen Aspekt zu berücksichtigen, der bereits im Theorieteil ausführlich diskutiert wurde. Es handelt sich dabei um die im Zeitverlauf aufgrund der Akkumulation von ziellandspezifischem Humankapital stattfindende Einkommensassimilierung der Einwanderer. Dieser Assimilierungsprozess wird in der Literatur über die Aufenthaltsdauer der Ausländer (YSM) gemessen. Mit zunehmender Aufenthaltsdauer ist aufgrund der Investitionen in ziellandspezifisches Humankapital mit einer Einkommensverbesserung der Ausländer zu rechnen, d.h. es ist von einem positiven Zusammenhang zwischen der Aufenthaltsdauer und der Einkommenshöhe auszugehen, wobei auch hier analog zur Arbeitserfahrung mit einem konkaven Verlauf des Aufenthaltsdauer-Einkommensprofils zu rechnen ist. ${ }^{235} \mathrm{Da}$ rund zwei Drittel der Frauen und drei Viertel der Männer seit mindestens $10 \mathrm{Jah}-$ ren in der Schweiz leben (siehe TAB. 8.7), ist allerdings nicht auszuschliessen, dass der Assimilierungseffekt gering bzw. nicht-signifikant ausfällt.

234 Einschränkend muss allerdings darauf hingewiesen werden, dass unter dem Wirtschaftszweig 'sonstige Dienstleistungen' sowohl Branchen, die ein höheres als auch Branchen die ein tieferes Qualifikationsprofil erfordern, zusammengefasst sind (siehe TAB. A9.17).

235 Analog zur Berufserfahrung, musste aufgrund hoher Multikollinearität ebenfalls hinsichtlich der Aufenthaltsdauer auf eine Berücksichtigung der quadrierten Aufenthaltsdauer verzichtet werden. 
Analog zur Analyse der Beschäftigungsperformance werden schliesslich zwei Dummy-Variablen für Nordeuropäer (NTHEU_IC) und Südeuropäer (STHEU) verwendet, wobei die Nicht-Europäer (OTHCT) als Basiskategorie dienen, um nationalitätenspezifischen Unterschieden in der Einkommensperformance Rechnung zu tragen. Eine Zusammenfassung der Modellspezifikation bzw. Definition der zur Schätzung der Einkommensfunktion verwendeten Variablen findet sich in TAB. 10.3.

Tab. 10.3: Modellspezifikation: Einkommensfunktion (ohne Heckman-Korrektur)

\begin{tabular}{|c|c|c|c|}
\hline Variablenname & Definition & Variablenname & Definition \\
\hline LNINCGM & $\begin{array}{l}\text { Logarithmiertes monatliches } \\
\text { Bruttoerwerbseinkommen }\end{array}$ & SECTOR3 & $\begin{array}{l}\text { Beschäftigung im höherquali- } \\
\text { fizierten tertiären Sektor }\end{array}$ \\
\hline$S C H$ & Anzahl Schujahre & $Y S M$ & Aufenthaltsdauer in Jahren \\
\hline$E X P E$ & $\begin{array}{l}\text { Anzahl Jahre an } \\
\text { Arbeitserfahrung }\end{array}$ & UNPL & $\begin{array}{l}\text { Kantonale } \\
\text { Arbeitslosenquoten }\end{array}$ \\
\hline$E M W M$ & $\begin{array}{l}\text { Berufliche Stellung mit } \\
\text { Vorgesetztenfunktion }\end{array}$ & NTHEU_IC & $\begin{array}{l}\text { Dummy-Variable für } \\
\text { Nordeuropäer }\end{array}$ \\
\hline$E M B M$ & $\begin{array}{l}\text { Berufliche Stellung mit Un- } \\
\text { ternehmensleitungsaufgaben }\end{array}$ & STHEU & $\begin{array}{l}\text { Dummy-Variable für } \\
\text { Südeuropäer }\end{array}$ \\
\hline
\end{tabular}

Quelle: Eigene Darstellung

Abschliessend gilt es noch auf eine vorteilhafte Eigenschaft semi-logarithmischer Modelle hinzuweisen. Im Rahmen dieser Modelle sind die Koeffizienten der erklärenden Variablen durch $\beta_{i}=\ln \left(1+b_{i}\right)$ gegeben, wobei $b_{i}$ der prozentualen Veränderung der endogenen Variablen auf eine Veränderung der exogenen Variablen entspricht. Bei kleinen Werten nahe null ist $\beta_{i} \approx b_{i}$. Für grössere Werte von $\beta_{i}$ gilt dies jedoch nicht. In diesen Fällen ist der Anti-Logarithmus zu bilden, d.h. $\exp (\beta)-1=b_{i}$, um eine Schätzung für $b_{i} z u$ erhalten.

\subsubsection{Selektivitäts-Bias Problematik}

Im Rahmen der bisherigen Ausführungen blieb ein Problempunkt ausgeklammert, der sich darauf bezieht, dass Einkommensdaten nur für Erwerbstätige verfügbar sind, was einen sog. Stichproben-Auswahlfehler (sample-selection bias) zur Folge haben kann. Es lässt sich zeigen, dass der Stichproben-Auswahlfehler i.d.R. zu Verzerrungen der geschätzten Regressionskoeffizienten in der Einkommensfunktion führt. ${ }^{236}$ Daher müsste die Einkommensfunktion durch ein Modell zur Erwerbstätigkeitswahrscheinlichkeit ergänzt werden. ${ }^{237}$ Durch diese Vorgehens-

236 Siehe DIEKMANN/ENGELHARDT (1994).

237 Siehe KUGLER (1988). 
weise lassen sich die Auswirkungen der Endogenität der Erwerbstätigkeit auf die Einkommensfunktion berücksichtigen. HECKMAN $(1974,1976 a, 1979)$ folgend, wird dem Einfluss der Erwerbstätigkeitsentscheidung i.a. durch die Aufnahme einer Selektionskorrekturvariablen $\lambda$, der sog. Heckman-Korrektur, in der Einkommensfunktion Rechnung getragen.

Die Werte der Variablen $\lambda$ werden mit zunehmender Erwerbstätigkeitswahrscheinlichkeit kleiner, so dass die Selektionskorrekturvariable in erster Linie für Wirtschaftssubjekte mit einer relativ geringen Erwerbstätigkeitswahrscheinlichkeit von Bedeutung ist. Aufgrund der sehr hohen Erwerbstätigenquoten der Männer, wird die Heckman-Korrektur daher lediglich in den Einkommensfunktionen der Frauen berücksichtigt. ${ }^{238}$ Der Koeffizient der Variablen $\lambda$ in der Einkommensregression zeigt die Richtung der Selektionsverzerrung an. Im Falle eines negativen Koeffizienten stellt das beobachtete Einkommen eine Unterschätzung, im Falle eines positiven Wertes eine Überschätzung des auf dem Markt erzielbaren Einkommens dar. Mit einer Selektionsverzerrung ist vor allem dann zu rechnen, wenn unbeobachtete Merkmale, die einen Einfluss auf die Erwerbstätigkeitswahrscheinlichkeit besitzen, mit der Höhe des Markteinkommens korreliert sind.

Unter Berücksichtigung dieser Überlegungen lässt sich die sog. Heckit-Procedure somit wie folgt skizzieren: In einem ersten Schritt wird ein Probit-Modell für die Erwerbstätigkeitswahrscheinlichkeit der Frauen geschätzt, aus dem sich die Selektionskorrekturvariable $\lambda$ ermitteln lässt. In einem zweiten Schritt wird diese Variable zur Bereinigung der Einkommensfunktion der Frauen verwendet. In Anlehnung an die Arbeitsmarkttheorie ergibt sich folgende Spezifikation für das Probit-Modell. Als erklärende Variable dient, wie bereits erwähnt, die Erwerbstätigkeitswahrscheinlicheit (EMPL). Analog zur Erwerbslosigkeitswahrscheinlichkeit handelt es sich dabei um eine dichotome Variable. Sie nimmt einen Wert von 1 an, wenn die betrachtete Person erwerbstätig ist und einen Wert von 0 in allen anderen Fällen. Um eine konsistente Analyse der Einkommensperformance zu gewährleisten, wird Erwerbstätigkeit in diesem Zusammenhang ebenfalls als Vollzeiterwerbstätigkeit definiert.

Hinsichtlich der erklärenden Variablen gilt es zwischen zwei Kategorien zu unterscheiden. In der ersten Kategorie finden sich diejenigen Variablen, die den Marktlohn bestimmten. Es handelt sich dabei im wesentlichen um diejenigen Variablen, die bereits in der Einkommensfunktion berücksichtigt wurden. Die zweite Kategorie umfasst demgegenüber die Determinanten des Anspruchslohns. Diese Überlegungen verdeutlichen, dass von der Annahme ausgegangen wird, dass die Nichterwerbstätigkeit der Frauen, d.h. EMPL=0, das Ergebnis einer freiwilligen Partizipationsentscheidung ist, die sich aus dem Vergleich von Anspruchs- und Marktlohn ergibt. Es wird also nicht unterstellt, dass die Nichterwerbstätigkeit

238 Siehe GoldER (1998) und GoLDER/STRAUBHAAR (1998). 
ganz oder teilweise auf diskriminierende Einstellungspraktiken der Arbeitgeber zurückzuführen ist. Dieser Punkt ist für die Analyse der Einkommensdiskriminierung im nachfolgenden Abschnitt von Bedeutung.

In einem ersten Schritt wird nun auf die Variablen der ersten Kategorie eingegangen. ${ }^{239}$ Als erklärende Variablen dienen wie in der Einkommensfunktion die Ausbildung ( $\boldsymbol{S C H}$ ) und die Arbeitserfahrung (EXPE). Analog zur Einkommensfunktion ist hinsichtlich der Ausbildung mit einem positiven Effekt der Qualifikationsprofile auf die Erwerbstätigkeitswahrscheinlichkeit zu rechnen, da eine bessere Ausbildung i.a. auch die Chancen erhöht, eine Arbeitsstelle zu finden. Da im vorliegenden Fall aber nur Vollzeiterwerbstätige als Erwerbstätige definiert sind, besteht die Möglichkeit, dass mit zunehmendem Einkommen der Substitutionseffekt überwiegt und daher eine Verringerung des Arbeitsangebots sowie der Erwerbstätigkeitswahrscheinlichkeit stattfindet. Hinsichtlich der Arbeitserfahrungsvariablen lassen sich ebenfalls keine eindeutigen Aussagen machen, da analog zur Einkommensfunktion mit einem konkaven Erwerbstätigkeitsprofil zu rechnen ist. ${ }^{240}$

Neben diesen personenspezifischen, mikroökonomischen Determinanten der Erwerbstätigkeitswahrscheinlichkeit wird als Indikator für die wirtschaftliche Lage wiederum die kantonale Arbeitslosenquote (UNPL) verwendet. Analog zu den Überlegungen im Rahmen der Analyse der Erwerbslosigkeitswahrscheinlichkeit ist davon auszugehen, dass zwischen der Höhe der kantonalen Arbeitslosenquoten und der Erwerbstätigkeitswahrscheinlichkeit ein negativer Zusammenhang besteht. Eine durch die schlechte wirtschaftliche Lage bedingte Lohnzurückhaltung der Arbeitnehmer und Gewerkschaften kann allerdings auch zu einer Erhöhung der Erwerbstätigkeitswahrscheinlichkeit führen. Ein positiver Zusammenhang ergibt sich auch unter Berücksichtigung der sog. 'added worker'-Hypothese, die im Rahmen einer Haushaltsperspektive impliziert, dass eine Tendenz zum Ausgleich rezessionsbedingter Einkommenseinbussen durch die zusätzliche Erwerbstätigkeit bisher nichterwerbstätiger Haushaltsmitglieder besteht. Analog zur Einkommensfunktion werden schliesslich ebenfalls Dummy-Variablen für Nordeuropäer (NTHEU_IC) und Südeuropäer (STHEU) verwendet, um nationalitätenspezifischen Unterschieden in den Erwerbstätigkeitswahrscheinlichkeiten Rechnung zu tragen.

Nachfolgend werden nun noch die Variablen der zweiten Kategorie diskutiert, die der Erklärung des Anspruchslohns dienen. Als erste Variable werden die logarithmierten monatlichen Haushaltsresidualeinkommen ( $\left.L N \_H H I N C\right)$ verwendet, die sich aus der Differenz der monatlichen Totaleinkommen der Haushalte

239 Auf die Verwendung der Variablen, EMWM, EMBM und SECTOR3 musste im Probit-Modell zur Berechnung der HECKMAN-Korrektur verzichtet werden, da zu diesen Variablen für die Nicht-Erwerbstätigen z.T. keine Informationen vorliegen.

Siehe FRANZ (1991). 
und den monatlichen Bruttoerwerbseinkommen der Zielpersonen berechnen. Analog zur Analyse der Erwerbslosigkeitswahrscheinlichkeit impliziert ein zunehmendes Haushaltsresidualeinkommen eine Verringerung der Erwerbstätigkeitswahrscheinlichkeit, da die Opportunitätskosten der Arbeit steigen. Wie aus der deskriptiven Analyse in Abschnitt 9.1 hervorging, ist der Anteil teilzeiterwerbstätiger Frauen vergleichsweise hoch, so dass aufgrund des Substitutionseffektes mit zunehmendem Haushaltseinkommen auch mit verstärkter Teilzeiterwerbstätigkeit der Frauen zu rechnen ist, was im vorliegenden Fall einem negativen Effekt auf die Erwerbstätigkeitswahrscheinlichkeit gleichkommt.

Schliesslich wird davon ausgegangen, dass die Existenz von Kindern einen Einfluss auf die Erwerbstätigkeitswahrscheinlichkeit der Eltern besitzt. In der ProbitRegression werden hierzu, nach Altersgruppen gegliedert, drei verschiedene Variablen verwendet. Die erste Variable umfasst die Zahl der Kinder im Alter bis 6 Jahre (KID_6), die zweite die Zahl der Kinder im Alter zwischen 7 und 14 Jahren (KID7_14) und die dritte Variable schliesslich die Zahl der Kinder im Alter zwischen 15 und 25 Jahren (KID15_25). Es ist zu vermuten, dass die Opportunitätskosten der Arbeit und damit der Anspruchslohn mit abnehmendem Alter der Kinder steigt. Dies impliziert, dass sich mit zunehmendem Alter der Kinder der negative Effekt auf die Erwerbstätigkeitswahrscheinlichkeit verringert. In TAB. 10.4 findet sich eine Zusammenfassung der für die Heckman-Korrektur verwendeten Variablen.

Tab. 10.4: Modellspezifikation: Heckman-Korrektur

\begin{tabular}{|c|c|c|c|}
\hline Variablenname & Definition & Variablenname & Definition \\
\hline$E M P L$ & $\begin{array}{l}\text { Logarithmiertes Bruttojahre- } \\
\text { serwerbseinkommen }\end{array}$ & STHEU & $\begin{array}{l}\text { Dummy-Variable für } \\
\text { Südeuropäer }\end{array}$ \\
\hline $\mathrm{SCH}$ & Anzahl Schuljahre & $L N \_H H I N C$ & $\begin{array}{l}\text { Logarithmiertes monatliches } \\
\text { Haushaltsresidualeinkommen }\end{array}$ \\
\hline$E X P E$ & $\begin{array}{l}\text { Anzahl Jahre an } \\
\text { Arbeitserfahrung }\end{array}$ & KID_6 & $\begin{array}{l}\text { Anzahl Kinder im Alter bis } 6 \\
\text { Jahre }\end{array}$ \\
\hline UNPL & $\begin{array}{l}\text { Kantonale } \\
\text { Arbeitslosenquoten }\end{array}$ & KID7_14 & $\begin{array}{l}\text { Anzahl Kinder im Alter zwi- } \\
\text { schen } 7 \text { und } 14 \text { Jahren }\end{array}$ \\
\hline NTHEU_IC & $\begin{array}{l}\text { Dummy-Variable für } \\
\text { Nordeuropäer }\end{array}$ & KID15_25 & $\begin{array}{l}\text { Anzahl Kinder im Alter zwi- } \\
\text { schen } 15 \text { und } 25 \text { Jahren }\end{array}$ \\
\hline
\end{tabular}

Quelle: Eigene Darstellung

Abschliessend ist allerdings darauf hinzuweisen, dass die Verwendung der Heckman-Korrektur nicht unproblematisch ist. PUHANI (1997:13) gelangt in einem Übersichtsartikel zum Schluss, dass theoretische Überlegungen sowie die Ergebnisse verschiedener Monte Carlo Studien "... cast doubt on the omnipotence implicitly ascribed by many applied researchers to Heckman's (1976[a], 1979) two- 
step estimator". Einer der zentralen Kritikpunkte bezieht sich auf die Multikollinearität zwischen den Variablen der beiden Schätzgleichungen. Empirische Studien sind oftmals mit dem Problem behaftet, dass eine hohe Korrelation zwischen den exogenen Variablen der Selektions- und der Hauptgleichung besteht. Daraus ergibt sich ein Kollinearitätsproblem zwischen der Selektionskorrekturvariablen und den Regressoren der Hauptgleichung, das zu ineffizienten Schätzern führen kann. Wie PUHANI betont, ist die Verwendung der Heckit-Procedure zur Korrektur des Stichproben-Auswahlfehlers dann am erforderlichsten, wenn erstens eine hohe Korrelation zwischen den Störtermen der Selektions- und der Hauptgleichung, d.h. zwischen der Erwerbstätigkeits- und der Einkommensfunktion besteht und wenn zweitens ein hoher Grad an Zensierung der Daten vorliegt. In genau diesen Fällen ist der HECKMAN-Schätzer jedoch besonders ineffizient, so dass MLESchätzungen robustere Ergebnisse ermöglichen. ${ }^{241}$

Aufgrund dieser Überlegungen ist eine fallweise Überprüfung der Vorteilhaftigkeit der Verwendung der HECKMAN-Korrektur zu empfehlen. PUHANI schlägt vor, vorab zu überprüfen, ob Kollinearitätsprobleme vorliegen. Ist dies der Fall, dann gestaltet sich die MLE-Schätzung nicht nur einfacher, sondern kann, wie bereits angesprochen, auch zu robusteren Schätzergebnissen führen. Liegen demgegenüber keine Kollinearitätsprobleme vor, dann kann die HECKMAN-Korrektur zu besseren Schätzergebnissen führen.

\subsubsection{EMPIRISCHE ERGEBNISSE}

Drei Fragestellungen sind bei der empirischen Analyse der Einkommensperformance in- und ausländischer Arbeitnehmer von Interesse: (i) Wieviel verdienen Ausländer im Vergleich zu Inländern, und gibt es Unterschiede zwischen den verschiedenen Nationalitätengruppen? (ii) Liegen diese Unterschiede in individuellen Charakteristika der Ausländer (z.B. dem Ausbildungsniveau) oder deren Ausländereigenschaft (Diskriminierung) begründet? (iii) Findet über die Zeit eine Angleichung der Einkommen von Ausländern und Schweizern statt (Assimilierung)? Die erste und dritte Frage werden in diesem Abschnitt, die dritte in Abschnitt 10.3.2 beantwortet.

Die Untersuchungen dieses Abschnitts stützen sich auf die empirischen Ergebnisse zur MINCER'SCHEN Einkommensfunktion aus Gl. (10.1). Vorgängig sind in den TAB. 10.5 und 10.6 einige deskriptive Statistiken zu den für die HECKMANKorrektur sowie für die Einkommensfunktion verwendeten Variablen abgebildet. Wie aus TAB. 10.5 hervorgeht, besitzen die ausländischen Frauen eine deutlich höhere Erwerbstätigkeitswahrscheinlichkeit als die inländischen Frauen. Mit Blick auf TAB. 9.7 lässt sich dieses Ergebnis u.a. auf einen höheren Anteil vollzeiterwerbstätiger Ausländerinnen zurückführen. Aus TAB. 10.5 geht ebenfalls hervor,

241 Siehe PUHANI (1997). 
dass die Inländerinnen einerseits eine längere Ausbildungszeit, andererseits aber eine geringere Berufserfahrung besitzen. Hinsichtlich des Haushaltsresidualeinkommens zeigt sich schliesslich, dass dieses bei den Inländerinnen rund ein Viertel über jenem der Ausländerinnen liegt.

Tab. 10.5: Deskriptive Statistiken: Heckman-Korrektur

\begin{tabular}{|l|c|c|l|c|c|}
\hline \multicolumn{1}{|c|}{ Variablen } & $\begin{array}{c}\text { Inländische } \\
\text { Frauen }\end{array}$ & $\begin{array}{c}\text { Ausländische } \\
\text { Frauen }\end{array}$ & Variablen & $\begin{array}{c}\text { Inländische } \\
\text { Frauen }\end{array}$ & $\begin{array}{c}\text { Ausländische } \\
\text { Frauen }\end{array}$ \\
\hline Stichproben- & 6652 & 802 & Stichproben- & 6652 & 802 \\
grösse & 0.38 & 0.47 & Srösse & & \\
EMPL & $(0.48)$ & $(0.50)$ & & & 0.46 \\
SCH & 10.17 & 9.85 & LN_HHINC & 6.30 & 6.03 \\
& $(2.09)$ & $(2.85)$ & & $(3.74)$ & $(3.71)$ \\
EXPE & 23.40 & 23.64 & KID_6 & 0.32 & 0.29 \\
& $(12.65)$ & $(11.48)$ & & $(0.68)$ & $(0.58)$ \\
UNPL & 4.12 & 4.84 & KID7_14 & 0.33 & 0.37 \\
& $(1.85)$ & $(1.97)$ & & $(0.72)$ & $(0.69)$ \\
NTHEU_IC & & 0.33 & KIDI5_25 & 0.38 & 0.26 \\
& & & & $(0.48)$ & $(0.62)$ \\
\hline
\end{tabular}

Quelle: SAKE (1995), eigene Berechnungen

Anmerkungen: Für stetige Variablen sind die Standardfehler in Klammern angegeben

Der Blick auf die deskriptiven Statistiken zur Einkommensfunktion in TAB. 10.6 zeigt, dass die Inländer im Mittel höhere Erwerbseinkommen erreichen als die Ausländer, wobei zwischen den Männern und Frauen grosse Unterschiede bestehen. ${ }^{242}$ Während dies ebenfalls für die Ausbildung gilt, trifft für die Berufserfahrung genau das Gegenteil zu. Deutliche Unterschiede zwischen Männern und Frauen lassen sich auch hinsichtlich der beruflichen Stellung beobachten. Aufgrund der Heterogenität innerhalb der Sektorvariablen (siehe TAB. A9.17 in Anhang 1), lassen sich jedoch keine eindeutigen Aussagen über den Effekt dieser Variablen machen. Abschliessend ist zu vermuten, dass der Assimilierungseffekt wegen der relativ langen mittleren Aufenthaltsdauer der Ausländer gering ausfallen wird.

242 Die Differenz der logarithmierten Werte lässt sich annähernd als prozentualer Unterschied interpretieren, d.h. der geometrische Mittelwert des Einkommens der inländischen Männer liegt rund 15 Prozent über jenem der ausländischen Männer. Bei grösseren logarithmischen Differenzen stellt dies allerdings nur noch eine grobe Näherung dar. Allgemein gilt, dass $\exp (\Delta \ln x)-1 \equiv \Delta x / x$, so dass der effektive prozentuale Einkommensunterschied 15.5 Prozent beträgt. 
Tab. 10.6: Deskriptive Statistiken: Einkommensfunktion

\begin{tabular}{|c|c|c|c|c|}
\hline Variablen & $\begin{array}{c}\text { Inländische } \\
\text { Männer }\end{array}$ & $\begin{array}{c}\text { Inländische } \\
\text { Frauen }\end{array}$ & $\begin{array}{c}\text { Ausländische } \\
\text { Männer }\end{array}$ & $\begin{array}{c}\text { Ausländische } \\
\text { Frauen }\end{array}$ \\
\hline Stichprobengrösse & 4733 & 2500 & 757 & 378 \\
\hline LNINCGM & $\begin{array}{c}8.66 \\
(0.37)\end{array}$ & $\begin{array}{c}8.33 \\
(0.35)\end{array}$ & $\begin{array}{c}8.51 \\
(0.37)\end{array}$ & $\begin{array}{c}8.20 \\
(0.38)\end{array}$ \\
\hline $\mathrm{SCH}$ & $\begin{array}{l}10.94 \\
(2.61)\end{array}$ & $\begin{array}{l}10.46 \\
(2.25)\end{array}$ & $\begin{array}{l}10.34 \\
(3.09)\end{array}$ & $\begin{array}{l}10.07 \\
(2.90)\end{array}$ \\
\hline$E X P E$ & $\begin{array}{c}21.40 \\
(11.15)\end{array}$ & $\begin{array}{c}18.95 \\
(11.52)\end{array}$ & $\begin{array}{c}24.57 \\
(10.65)\end{array}$ & $\begin{array}{l}21.71 \\
(9.82)\end{array}$ \\
\hline$E M W M$ & 0.31 & 0.20 & 0.23 & 0.14 \\
\hline$E M B M$ & 0.14 & 0.06 & 0.09 & 0.05 \\
\hline SECTOR3 & 0.44 & 0.59 & 0.26 & 0.49 \\
\hline$U N P L$ & $\begin{array}{c}3.98 \\
(1.79)\end{array}$ & $\begin{array}{c}4.17 \\
(1.84)\end{array}$ & $\begin{array}{c}4.66 \\
(1.92)\end{array}$ & $\begin{array}{c}4.77 \\
(2.03)\end{array}$ \\
\hline$Y S M$ & & & $\begin{array}{c}17.51 \\
(10.70)\end{array}$ & $\begin{array}{l}14.59 \\
(9.65)\end{array}$ \\
\hline NTHEU_IC & & & 0.30 & 0.34 \\
\hline STHEU & & & 0.48 & 0.44 \\
\hline
\end{tabular}

Quelle: SAKE (1995), eigene Berechnungen

Anmerkungen: Für stetige Variablen sind die Standardfehler in Klammern angegeben

Bevor die Schätzergebnisse der Einkommensfunktion diskutiert werden, folgt analog zur empirischen Analyse der Beschäftigungsperformance eine Überprüfung der Parameterstabilität. Da die Analyse der Einkommensperformance auf OLSSchätzungen basiert, lässt sich die Hypothese der Parameterstabilität mit einem sog. 'Chow-Test' überprüfen. ${ }^{243}$ Es zeigt sich, dass die Hypothese der Parameter-

243 CHOw (1960). Im Rahmen des Chow-Tests werden die Einkommensfunktionen getrennt geschätzt, so dass sich die Quadratsummen der Residuen der beiden Teilstichproben berechnen lassen. Bei Einheitlichkeit der Koeffizienten ist zu erwarten, dass sich die Modellanpassung nicht entscheidend verbessern lässt, wenn die beiden Teilstichproben getrennt betrachtet werden. Die Quadratsumme der Residuen wird dann nicht wesentlich höher sein als die Quadratsumme der Residuen der beiden Schätzung der Teilstichproben zusammen. Ist demgegenüber die Einheitlichkeit der Koeffizienten nicht gegeben, wird die Residualquadratsumme der gepoolten Stichprobe signifikant über der Summe der beiden Quadratsummen der Residuen der beiden Teilstichproben liegen [siehe GUJARATI (1995)]. Die Berechnung der Teststatistiken in Tab. 10.7 beruht auf einer verallgemeinerten Form des Chow-Test, da die Annahme identischer Varianzen der Störterme in den Regressionen der beiden Teilstichproben aufgegeben wurde [siehe GREENE $(1995,1996)]$. 
stabilität klar abgelehnt werden muss. Aus diesem Grund erfolgen analog zur Analyse der Erwerbslosigkeitswahrscheinlichkeit getrennte Schätzungen der Einkommensfunktionen für in- und ausländische Männer und Frauen.

Tab. 10.7: Chow-Test auf Parameterstabilität

\begin{tabular}{|l|c|c|}
\hline & Teststatistik & Tabellenwert \\
\hline $\begin{array}{l}\text { Schweizer - } \\
\text { Ausländer } \\
\text { Schweizer (Männer) - } \\
\begin{array}{l}\text { Schweizer(Frauen) } \\
\text { Ausländer (Männer) - } \\
\text { Ausländer(Frauen) }\end{array}\end{array}$ & 120.4 & 15.51 \\
\hline
\end{tabular}

Quelle: SAKE (1995), eigene Berechnungen

Anmerkungen: Der Chow Test folgt einer $\chi^{2}$-Verteilung, wobei die Zahl der Freiheitsgrade der Zahl der Restriktionen entspricht. Die Tabellenwerte sind auf dem Signifikanzniveau von 5 Prozent ausgewiesen

Da für die Frauen zur Eliminierung des Stichprobenauswahlfehlers eine HECKMAN-Korrektur durchgeführt wurde, erfolgt die Präsentation der empirischen Ergebnisse getrennt nach Geschlecht. In TAB. 10.8 finden sich die Schätzergebnisse der Einkommensfunktion für die in- und ausländischen Männer. Erwartungsgemäss sind die Koeffizienten der Ausbildungs- und Berufserfahrungsvariablen sowie der Variablen zur beruflichen Stellung signifikant positiv. Die Insignifikanz der Branchenvariablen bei den ausländischen Männern lässt sich damit begründen, dass aufgrund der Heterogenität innerhalb dieser Variablen gegenläufige Effekte bestehen, die sich gegenseitig aufheben.

Desweiteren zeigt sich, dass die wirtschaftliche Lage weder bei den Inländern noch bei den Ausländern einen Einfluss auf die Einkommenshöhe besitzt. Der nicht-signifikante Effekt der Aufenthaltsdauer ist darauf zurückzuführen, dass der Assimilierungseffekt aufgrund der im Mittel relativ langen Aufenthaltsdauer nur noch sehr gering ausfällt. Die Berücksichtigung nationalitätenspezifischer Unterschiede zeigt schliesslich, dass Nordeuropäer und Südeuropäer gegenüber den Nicht-Europäern ceteris paribus einen signifikanten Einkommensvorsprung von 21 Prozent bzw. 9 Prozent aufweisen. Dieses Ergebnis lässt sich mit Blick auf die deskriptive Analyse in Kapitel 9 sowie die deskriptiven Statistiken in TAB. 10.6 auf Unterschiede in den individuellen Charakteristika zurückführen. 
Tab. 10.8: OLS-Regressionen der Einkommensfunktion, Männer

\begin{tabular}{|c|c|c|}
\hline Variablen & Inländer & Ausländer \\
\hline Stichprobengrösse & 4733 & 757 \\
\hline CONSTANT & $\begin{array}{r}7.696 * * * \\
(310.63)\end{array}$ & $\begin{array}{r}7.743 * * * \\
(105.17)\end{array}$ \\
\hline $\mathrm{SCH}$ & $\begin{array}{r}0.057 * * * \\
(29.73)\end{array}$ & $\begin{array}{r}0.044 * * * \\
(7.48)\end{array}$ \\
\hline$E X P E$ & $\begin{array}{r}0.011 * * * \\
(24.04)\end{array}$ & $\begin{array}{r}0.005^{* *} \\
(2.43)\end{array}$ \\
\hline$E M W M$ & $\begin{array}{r}0.144 * * * \\
(15.81)\end{array}$ & $\begin{array}{r}0.129 * * * \\
(5.10)\end{array}$ \\
\hline$E M B M$ & $\begin{array}{r}0.310^{* * * *} \\
(21.08)\end{array}$ & $\begin{array}{r}0.363 * * * \\
(7.19)\end{array}$ \\
\hline SECTOR3 & $\begin{array}{r}0.010 * * * \\
(11.33)\end{array}$ & $\begin{array}{c}0.034 \\
(1.15)\end{array}$ \\
\hline UNPL & $\begin{array}{r}-0.003 \\
(1.47)\end{array}$ & $\begin{array}{r}-0.005 \\
(0.92)\end{array}$ \\
\hline YSM & & $\begin{array}{r}0.002 \\
(1.06)\end{array}$ \\
\hline NTHEU_IC & & $\begin{array}{r}0.209 * * * \\
(5.96)\end{array}$ \\
\hline STHEU & & $\begin{array}{r}0.091 * * * \\
(3.15)\end{array}$ \\
\hline Adj. $-R^{2}$ & 0.402 & 0.421 \\
\hline
\end{tabular}

Quelle: SAKE (1995), eigene Berechnungen

Anmerkungen: Absolute $t$-Werte in Klammern sind heteroskedastizitätskorrigiert [siehe WHITE (1980)]

* bedeutet ein Signifikanzniveau von 10 Prozent, ** bedeuten ein Signifikanzniveau von 5 Prozent, *** bedeuten ein Signifikanzniveau von 1 Prozent

Die Analyse der Schätzergebnisse der Einkommensfunktion für die in- und ausländischen Frauen erfolgt in zwei Schritten. In einem ersten Schritt werden die Ergebnisse der Probit-Regression zur Erwerbstätigkeitswahrscheinlichkeit diskutiert. In einem zweiten Schritt folgt dann unter Berücksichtigung der Selektionskorrekturvariablen die Erörterung der Ergebnisse zur Einkommensfunktion. Aus TAB. 10.9 geht hervor, dass eine weitgehende qualitative Übereinstimmung der Schätzergebnisse zwischen den in- und ausländischen Frauen besteht, wobei allerdings Unterschiede hinsichtlich der quantitativen Dimension der signifikanten Variablen bestehen.

Augenfällig in TAB. 10.9 ist, dass vom Ausbildungsniveau sowohl bei den in- als auch bei den ausländischen Frauen kein signifikanter Einfluss auf die Erwerbstä- 
tigkeitswahrscheinlichkeit ausgeht. Dieses Ergebnis lässt sich damit erklären, dass der positive Zusammenhang zwischen Bildungsniveau und Erwerbstätigkeitswahrscheinlichkeit abgeschwächt wird, weil der ebenfalls positive Zusammenhang zum Einkommen über einen Substitutionseffekt zu einer Verringerung des Arbeitsangebots führen kann. $\mathrm{Da}$ in TAB. 10.9 lediglich Vollzeiterwerbstätige als erwerbstätig gelten, ist der Effekt der Ausbildung auf die Erwerbstätigkeitswahrscheinlichkeit nicht eindeutig. Für die Ausländerinnen ist zudem denkbar, dass der nicht-signifikante Effekt durch die unvollständige Transferierbarkeit des $\mathrm{Hu}$ mankapitals bedingt ist.

Tab. 10.9: Probit-Regressionen zur Erwerbstätigkeitswahrscheinlichkeit, Frauen

\begin{tabular}{|c|c|c|c|c|}
\hline \multirow[t]{2}{*}{ Variablen } & \multicolumn{2}{|c|}{ Inländerinnen } & \multicolumn{2}{|c|}{ Ausländerinnen } \\
\hline & $\begin{array}{c}\text { Schätzwert } \\
\text { (abs. } t \text {-Werte) }\end{array}$ & $\begin{array}{c}\text { Marginale } \\
\text { Effekte }\end{array}$ & $\begin{array}{c}\text { Schätzwert } \\
\text { (abs. } t \text {-Werte) }\end{array}$ & $\begin{array}{c}\text { Marginale } \\
\text { Effekte }\end{array}$ \\
\hline $\begin{array}{l}\text { Stichproben- } \\
\text { grösse }\end{array}$ & \multicolumn{2}{|c|}{6652} & \multicolumn{2}{|c|}{802} \\
\hline CONSTANT & $\begin{array}{r}1.852^{* * *} \\
(15.19)\end{array}$ & 0.6548 & $\begin{array}{r}2.117^{* * *} \\
(6.66)\end{array}$ & 0.8420 \\
\hline $\mathrm{SCH}$ & $\begin{array}{r}0.001 \\
(0.12)\end{array}$ & 0.0004 & $\begin{array}{r}-0.021 \\
(1.07)\end{array}$ & -0.0085 \\
\hline$E X P E$ & $\begin{array}{r}-0.044 * * * \\
(26.78)\end{array}$ & -0.0155 & $\begin{array}{r}-0.033^{* * *} \\
(6.72)\end{array}$ & -0.0132 \\
\hline UNPL & $\begin{array}{r}0.009 \\
(0.82)\end{array}$ & 0.0030 & $\begin{array}{r}-0.035 \\
(1.42)\end{array}$ & 0.0140 \\
\hline$L N \_H H I N C$ & $\begin{array}{r}-0.122 * * * \\
(23.92)\end{array}$ & -0.0432 & $\begin{array}{r}-0.110^{* * *} \\
(7.98)\end{array}$ & -0.0439 \\
\hline KID_6 & $\begin{array}{r}-1.135 * * * \\
(23.56)\end{array}$ & -0.4011 & $\begin{array}{r}-0.508^{* * *} \\
(5.40)\end{array}$ & -0.2020 \\
\hline KID7_14 & $\begin{array}{r}-0.573 * * * \\
(17.28)\end{array}$ & -0.2028 & $\begin{array}{r}-0.256^{* * *} \\
(3.41)\end{array}$ & -0.1019 \\
\hline KID15_25 & $\begin{array}{r}-0.260^{* * *} \\
(6.67)\end{array}$ & -0.0920 & $\begin{array}{r}-0.211^{* *} \\
(2.53)\end{array}$ & -0.0838 \\
\hline NTHEU_IC & & & $\begin{array}{r}-0.268^{*} \\
(1.89)\end{array}$ & -0.1065 \\
\hline STHEU & & & $\begin{array}{l}0.043 \\
(0.33)\end{array}$ & 0.0171 \\
\hline $\log -L$ & -28 & & -47 & \\
\hline Pseudo- $R^{2}$ & & & & \\
\hline
\end{tabular}

Quelle: SAKE (1995), eigene Berechnungen

Anmerkungen: * bedeutet ein Signifikanzniveau von 10 Prozent, ** bedeuten ein Signifikanzniveau von 5 Prozent, *** bedeuten ein Signifikanzniveau von 1 Prozent 
Insgesamt lässt sich hinsichtlich der Determinanten des Marktlohnes kein klarer Zusammenhang zur Erwerbstätigkeitswahrscheinlichkeit erkennen. Die Determinanten des Anspruchslohnes besitzen demgegenüber einen signifikant negativen Effekt auf die Erwerbstätigkeitswahrscheinlichkeit. Aus TAB. 10.9 geht schliesslich hervor, dass die Nordeuropäerinnen im Vergleich zu den Süd- und Nicht-Europäerinnen eine signifikant tiefere Erwerbstätigkeitswahrscheinlichkeit aufweisen. Dieses Ergebnis lässt sich mit Blick auf TAB. 9.7 damit erklären, dass ein relativ hoher Anteil der Nordeuropäerinnen lediglich teilzeiterwerbstätig ist. Dies trifft insbesondere im Vergleich zur Basiskategorie der Nicht-Europäerinnen zu.

Tab. 10.10: OLS-Regressionen der Einkommensfunktion, Frauen

\begin{tabular}{|c|c|c|}
\hline Variablen & Inländer & Ausländer \\
\hline Stichprobengrösse & 2500 & 378 \\
\hline CONSTANT & $\begin{array}{l}7.547 * * * \\
(217.99)\end{array}$ & $\begin{array}{r}7.463^{* * *} \\
(81.87)\end{array}$ \\
\hline$S C H$ & $\begin{array}{r}0.056 * * * \\
(19.04)\end{array}$ & $\begin{array}{r}0.044 * * * \\
(7.16)\end{array}$ \\
\hline EXPE & $\begin{array}{r}0.007 * * * \\
(11.59)\end{array}$ & $\begin{array}{r}0.006 * * \\
(2.24)\end{array}$ \\
\hline$E M W M$ & $\begin{array}{r}0.094 * * * \\
(6.29)\end{array}$ & $\begin{array}{r}0.207^{* * *} \\
(4.76)\end{array}$ \\
\hline$E M B M$ & $\begin{array}{r}0.179 * * * \\
(7.18)\end{array}$ & $\begin{array}{r}0.131^{* *} \\
(1.85)\end{array}$ \\
\hline SECTOR3 & $\begin{array}{r}0.165^{* * * *} \\
(13.04)\end{array}$ & $\begin{array}{r}0.182 * * * \\
(5.97)\end{array}$ \\
\hline UNPL & $\begin{array}{r}0.003 \\
(0.81)\end{array}$ & $\begin{array}{r}0.014^{*} \\
(1.84)\end{array}$ \\
\hline YSM & & $\begin{array}{r}0.001 \\
(0.43)\end{array}$ \\
\hline NTHEU_IC & & $\begin{array}{r}0.130 * * * \\
(2.99)\end{array}$ \\
\hline STHEU & & $\begin{array}{r}-0.043 \\
(1.05)\end{array}$ \\
\hline$L A M B D A$ & $\begin{array}{r}-0.118 * * * \\
(7.96)\end{array}$ & $\begin{array}{r}-0.101^{* *} \\
(2.11)\end{array}$ \\
\hline Adj. $-R^{2}$ & 0.270 & 0.437 \\
\hline
\end{tabular}

Quelle: SAKE (1995), eigene Berechnungen

Anmerkungen: Absolute t-Werte in Klammern sind heteroskedastizitätskorrigiert [siehe WHITE (1980)]

* bedeutet ein Signifikanzniveau von 10 Prozent, ** bedeuten ein Signifikanzniveau von 5 Prozent, *** bedeuten ein Signifikanzniveau von 1 Prozent 
Nach diesen Bemerkungen zu den Schätzergebnissen der Probit-Regression zur Berechnung der HECKMAN-Korrektur, finden sich in TAB. 10.10 die Schätzergebnisse zur Einkommensfunktion unter Berücksichtigung der Selektionskorrekturvariablen (LAMBDA). Der Vergleich zu den Ergebnissen in TAB. 10.8 für die männlichen Erwerbstätigen zeigt eine weitgehende qualitative Übereinstimmung in den Effekten der Einkommensdeterminanten. Der wesentliche Unterschied besteht darin, dass für die ausländischen Frauen ein signifikant positiver Zusammenhang zwischen der Höhe der Arbeitslosenquote und der Einkommenshöhe besteht. Dieses Ergebnis lässt sich auf den sog. 'added worker'-Effekt zurückführen.

Von Interesse in TAB. 10.10 ist vor allem die Selektionskorrekturvariable. Es zeigt sich, dass diese sowohl bei den inländischen als auch bei den ausländischen Frauen einen signifikant negativen Effekt auf die Einkommenshöhe besitzt. Gemäss den Überlegungen zu den Determinanten der Erwerbstätigkeitswahrscheinlichkeit folgt daraus, dass die Störterme der Markt- und Anspruchslohngleichung positiv korreliert sind. Bei identischen beobachtbaren Merkmalen werden Frauen mit einem potentiell höheren Einkommen auch einen höheren Anspruchslohn aufweisen und damit auf dem Arbeitsmarkt eher unterrepräsentiert sein. Das beobachtete Einkommen der erwerbstätigen Frauen unterschätzt daher das Einkommen der erwerbsfähigen Frauen. 244

\subsection{EMPIRISCHE ANALYSE DER EINKOMMENSDISKRIMINIERUNG}

Nachdem im vorangegangenen Abschnitt produktivitätsbezogene Faktoren zur Erklärung von Unterschieden in der Beschäftigungsperformance im Vordergrund standen, wird im Rahmen der nachfolgenden Ausführungen der Einfluss diskriminierungsbedingter Faktoren erörtert.

\subsubsection{METHODISCHE VORGEHENSWEISE}

Die Ausführungen in Kapitel 6 haben gezeigt, dass sich geschlechts- und/oder nationalitätenspezifische Einkommensunterschiede nicht nur auf produktivitätsbedingte sondern auch auf diskriminierungsbedingte Ursachen zurückführen lassen. Es gilt dabei allerdings zu bedenken, dass die Einkommensdifferenz zwischen den verschiedenen Gruppen nicht zwingend durch Diskriminierung bedingt sein muss, da Unterschiede in der Humankapitalausstattung, im Ausmass der Berufserfahrung oder in der Stabilität der Erwerbskarrieren zumindest einen Teil der Einkommensdiskrepanz zu erklären vermögen. Diese Faktoren wurden im Rahmen der Analyse der Determinanten der Einkommensfunktion bereits berücksichtigt. In der Regel wird die verbleibende Restgrösse, d.h. der nicht durch Ausstattungsun-

$244 \mathrm{Zu}$ ähnlichen Ergebnissen für die Schweiz gelangen auch KUGLER (1988) und DIEKMANN/ENGELHARDT (1994). 
terschiede erklärbare Teil des Einkommensdifferentials, als Näherungsgrösse für das Ausmass der Lohn- bzw. Einkommensdiskriminierung verwendet.

Es ist allerdings nicht unproblematisch, diesen unerklärten Teil der Einkommensdifferenz mit Diskriminierung gleichzusetzen, die gemäss den theoretischen Ausführungen in Kapitel 6 als Einkommensunterschied bei gleicher Produktivität definiert war. ${ }^{245}$ Erstens lässt sich die Produktivität i.a. nicht vollständig durch Ausstattungsmerkmale erfassen, was zu einer Über- oder Unterschätzung der Diskriminierungskomponente führen kann. Zweitens können Ausstattungsunterschiede teilweise durch vorhergehende Diskriminierungsprozesse bedingt sein, wenn z.B. Eltern trotz gleicher Begabung die Ausbildung der Söhne stärker fördern als jene der Töchter. Drittens geht aus TAB. 6.1 hervor, dass Einkommensdiskriminierung lediglich einen möglichen Diskriminierungstatbestand darstellt. Neben der Einkommensdiskriminierung bildet die in Kapitel 9 diskutierte Arbeitsmarktsegmentation einen zweiten wichtigen Diskriminierungstatbestand. Ungeachtet dieser Probleme besitzen Untersuchungen, die auf der Grundlage statistischer Methoden versuchen, Einkommensunterschiede über Ausstattungs- und Diskriminierungskomponenten zu erklären, aus wissenschaftlicher wie auch aus wirtschaftspolitischer Sicht einen wichtigen Erklärungs- und Informationswert.

Zur Analyse der Einkommensdiskriminierung wird i.a. eine Untersuchungsmethode verwendet, die auf die bedeutenden Beiträge von BLINDER (1973) und OAXACA (1973) zurückgeht. Der absolute Einkommensunterschied zwischen zwei Gruppen, einer nicht-benachteiligten und einer (angeblich) benachteiligten, in unserem Fall den Inländern und den Ausländern, wird in eine Diskriminierungskomponente und eine Ausstattungskomponente zerlegt. Als Ausgangspunkt der Analyse dient die im vorangegangenen Abschnitt behandelte MINCER'SCHE Einkommensfunktion, die für In- und Ausländer getrennt geschätzt wird. Die so ermittelten Koeffizienten bilden die Grundlage für die Simulation des Arbeitsmarktergebnisses, wobei sich das Ausmass der Diskriminierung aus der Differenz der vorhergesagten Werte der zu erklärenden Variablen für die beiden Gruppen der In- und Ausländer ergibt. Der Vorteil dieser Untersuchungsmethodik besteht zum einen darin, dass sie mit relativ geringem Aufwand verbunden ist und dass sie zum anderen im Vergleich zu den in Abschnitt 6.2 erwähnten Experimenten eine um ein Vielfaches höhere Fallzahl beinhaltet, so dass i.d.R. eine ausreichende Repräsentativität der Ergebnisse gewährleistet ist.

Im OAXACA/BLINDER-Modell liegt Diskriminierung dann vor, wenn sich die geschätzten Ausstattungseffekte, d.h. die Koeffizientenvektoren der In- und Ausländer, signifikant voneinander unterscheiden. Die empirische Messung der Einkommensdiskriminierung gestaltet sich im einzelnen wie folgt. Die exogenen Variablen werden jeweils zu ihrem Mittelwert (oder, im Falle von Dummy-Variab-

245 Siehe DIEKMANN/ENGELHARDT (1994). 
len, dem entsprechenden Stichprobenanteil) bewertet. Diese Werte werden mit den geschätzten Koeffizienten aus der Einkommensfunktion gewichtet, um daraus die prognostizierten (logarithmierten) Einkommen zu berechnen. Im Falle einer linearen Beziehung sowie unter Berücksichtigung einer Konstanten entsprechen die prognostizierten Einkommen genau den Mittelwerten der Stichprobe. ${ }^{246}$

Die Vorgehensweise lässt sich formal wie folgt darstellen. In einem ersten Schritt werden, wie bereits erwähnt, die nachfolgenden Einkommensfunktionen für Inund Ausländer getrennt geschätzt

$$
\begin{array}{ll}
\text { (10.3) } & \ln \mathrm{Y}_{\mathrm{ni}}=\mathrm{X}_{\mathrm{ni}} \beta_{\mathrm{ni}}+\lambda_{\mathrm{nf}} \gamma_{\mathrm{nf}}+\varepsilon_{\mathrm{ni}} \text { und } \\
\text { (10.4) } & \ln \mathrm{Y}_{\mathrm{fi}}=\mathrm{X}_{\mathrm{fi}} \beta_{\mathrm{fi}}+\lambda_{\mathrm{ff}} \gamma_{\mathrm{ff}}+\mathrm{Z}_{\mathrm{fi}} \alpha_{\mathrm{fi}}+\varepsilon_{\mathrm{fi}} \\
& \text { mit } \quad \mathrm{ni}=\text { inländische Männer (nm) und Frauen (nf) } \\
& \mathrm{fi}=\text { ausländische Männer (fm) und Frauen (ff) }
\end{array}
$$

wobei $\beta_{\mathrm{ni}}$ bzw. $\beta_{\mathrm{fi}}$ die Koeffizientenvektoren der für die In- und Ausländer identischen Variablen repräsentieren. $\alpha_{\mathfrak{i}}$ steht für den Koeffizientenvektor der ausländerspezifischen Variablen und $\gamma_{\mathrm{nf}}$ bzw. $\gamma_{\mathrm{ff}}$ für die Koeffizienten der Selektionskorrekturvariablen. Da für Kleinstquadratschätzer gilt, dass die geschätzte Regressionsgerade durch die Stichprobenmittelwerte verläuft, lassen sich die beiden obigen Gleichungen wie folgt umformulieren

$$
\begin{aligned}
& \ln \bar{Y}_{n i}=\bar{X}_{n i} \hat{\beta}_{n i}+\bar{\lambda}_{n f} \hat{\gamma}_{n f} \quad \text { und } \\
& \ln \bar{Y}_{f i}=\bar{X}_{f i} \hat{\beta}_{f}+\bar{\lambda}_{\mathrm{ff}} \hat{\gamma}_{\mathrm{ff}}+\bar{Z}_{\mathrm{f}} \hat{\alpha}_{\mathrm{f}},
\end{aligned}
$$

wobei $\beta_{n i}$ bzw. $\beta_{f i}, \hat{\alpha}_{f f}$ sowie $\hat{\gamma}_{n f}$ bzw. $\hat{\gamma}_{\mathrm{ff}}$ die Vektoren der geschätzten OLSKoeffizienten respräsentieren. Die Differenz der Mittelwerte der logarithmierten Einkommen der beiden Gruppen ergibt sich somit als

$$
\ln \bar{Y}_{n i}-\ln \bar{Y}_{f i}=\left(\bar{X}_{n i} \hat{\beta}_{n i}-\bar{X}_{f i} \hat{\beta}_{f i}\right)+\left(\bar{\lambda}_{n f} \hat{\gamma}_{n f}-\bar{\lambda}_{f f} \hat{\gamma}_{f f}\right)-\bar{Z}_{f f} \hat{\alpha}_{f f}
$$

Wird nun die Differenz der Koeffizientenvektoren für die nicht-ausländerspezifischen Variablen definiert als

$$
\Delta \hat{\beta}=\hat{\beta}_{n}-\hat{\beta}_{f} \text { so dass } \hat{\beta}_{f}=\hat{\beta}_{n}-\Delta \hat{\beta},
$$

so resultiert durch Substitution in Gl. (10.7) sowie einige Umformungen

246 VELLING (1995a). Es ist in diesem Zusammenhang allerdings darauf hinzuweisen, dass die Verwendung von Dummy-Variablen im Rahmen der OAXACA/BLINDER-Zerlegung mit Problemen verbunden ist, da die Ergebnisse von der Wahl der Referenzgruppe beeinflusst werden [siehe NIELSEN (1998)]. 


$$
\begin{aligned}
\ln \bar{Y}_{n i}-\ln \bar{Y}_{f i} & =\left(\hat{\beta}_{0 n i}-\hat{\beta}_{0 f i}\right)+\sum\left\{\bar{X}_{f i}\left(\hat{\beta}_{n i}-\hat{\beta}_{f i}\right)\right\}-\bar{Z}_{f f} \hat{\alpha}_{f f} \\
& \left.+\sum \hat{\beta}_{n i}\left(\bar{X}_{n i}-\bar{X}_{f i}\right)\right\}+\left(\bar{\lambda}_{n f} \hat{\gamma}_{n f}-\bar{\lambda}_{f f} \hat{\gamma}_{f f}\right)
\end{aligned}
$$

Das Einkommensdifferential zwischen In- und Ausländern lässt sich gemäss Gl. (10.9) wie folgt zerlegen.247 Die erste Zerlegungskomponente, der sog. Gruppeneffekt, setzt sich aus der Differenz der konstanten Terme sowie dem Anteil der Einkommensdifferenz zusammen, der auf divergierenden Koeffizienten beruht. In der Terminologie von BLINDER entspricht dieser Gruppeneffekt dem hypothetischen Einkommenszuwachs, den Ausländer erzielen könnten, wenn sie bei gleichbleibender Ausstattung dieselbe Einkommensstruktur aufweisen würden wie die Inländer. Der Gruppeneffekt lässt sich somit als Diskriminierungseffekt interpretieren, der dem Anteil des Einkommensdifferentials entspricht, der auf Diskriminierung zurückzuführen ist. BLINDER schreibt in diesem Zusammenhang, "... the latter sum, which exists only because the market evaluates differently the identical bundle of traits if possessed by members of different demographic groups, is a reflection of discrimination as much as the shift coefficient is". ${ }^{248}$ Schliesslich werden auch die ausländerspezifischen Variablen dem Diskriminierungseffekt zugerechnet. Diese Vorgehensweise lässt sich mit Blick auf die Überlegungen zum BECKER'SCHEN 'taste for discrimination' in Kapitel 6 darauf zurückführen, dass die Ausländereigenschaft ein vergleichsweise geringeres Einkommen nach sich zieht.

Der Ausstattungseffekt setzt sich aus den letzten beiden Termen in Gl. (10.9) zusammen. Die erste Komponente reflektiert das hypothetische Mehreinkommen der Ausländer, das diese erzielen könnten, wenn sie den Schweizern in Bezug auf die erklärenden Variablen gleichgestellt wären, d.h. wenn sie identische Ausstattungen aufweisen würden. Die zweite Komponente bezieht sich auf die Variable zur Korrektur der Selektionsverzerrung für weibliche Erwerbstätige. Diese wird dem Ausstattungseffekt zugerechnet, da von einer freiwilligen und unbeschränkten Partizipationsentscheidung der erwerbsfähigen Frauen ausgegangen wird. Würde hingegen unterstellt, dass erwerbswillige Frauen (in substantiellem Umfang) durch diskriminierende Einstellungspraktiken der Arbeitgeber von der Partizipation am Arbeitsmarkt ausgeschlossen würden, dann wäre diese Variable dem Diskriminierungseffekt zuzurechnen.

247 Da sich die Einkommenszerlegung im vorliegenden Fall nicht auf die absoluten, sondern auf die logarithmierten Einkommen bezieht, ergeben sich durch die Entlogarithmisierung die geometrischen Mittelwerte. Die Ausstattungs- und Diskrimierungskomponenten lassen sich dann approximativ als prozentuale Erhöhung oder Verminderung der geometrischen Gruppen-Mittelwerte interpretieren. Bei grösseren logarithmischen Differenzen stellt dies allerdings, wie bereits erwähnt, lediglich eine grobe Näherung dar.

BLINDER (1973: 438-439). 
Aus Gl. (10.9) geht hervor, dass die Ausstattungsunterschiede mit den geschätzten Koeffizienten der nicht-benachteiligten Gruppe gewichtet sind, während die Differenz der geschätzten Koeffizienten mit den durchschnittlichen Charakteristika der (angeblich) benachteiligten Gruppe gewichtet sind. Wird alternativ dazu in Gl. (10.7) nicht für $\beta_{f}$, sondern für $\beta_{n}$ substituiert, resultiert nach einigen Umformungen folgende alternative Formulierung der Einkommenszerlegung

$$
\begin{aligned}
\ln \bar{Y}_{n i}-\ln \bar{Y}_{f i} & =\left(\hat{\beta}_{0 n i}-\hat{\beta}_{0 f i}\right)+\sum\left\{\bar{X}_{n i}\left(\hat{\beta}_{n i}-\hat{\beta}_{f i}\right)\right\}-\bar{Z}_{f f} \hat{\alpha}_{f f} \\
& \left.+\sum \hat{\beta}_{f i}\left(\bar{X}_{n i}-\bar{X}_{f i}\right)\right\}+\left(\bar{\lambda}_{n f} \hat{\gamma}_{n f}-\bar{\lambda}_{f f} \hat{\gamma}_{f f}\right)
\end{aligned}
$$

In diesem Fall werden die durchschnittlichen Ausstattungsunterschiede mit den geschätzten Koeffizienten der (angeblich) benachteiligten Gruppe und die Differenz in den Koeffizienten mit den durchschnittlichen Charakteristika der nicht-benachteiligten Gruppe gewichtet. Die Wahl zwischen Gl. (10.9) und Gl. (10.10) zur Analyse des Ausmasses der Einkommensdiskriminierung stellt ein klassisches Indexnummerproblem dar. ${ }^{249} \mathrm{Zu}$ dessen Lösung werden in der Praxis i.a. beide Gleichungen zur Berechnung der Einkommenszerlegung verwendet, da unterstellt wird, dass sich durch diese beiden Messgrössen das Diskriminierungsintervall beschreiben lässt. In den nachfolgenden empirischen Ergebnissen zur Einkommensdiskriminierung werden daher die Ergebnisse beider Zerlegungsmethoden ausgewiesen.

Abschliessend gilt es noch, zwei Punkte zu erwähnen. Erstens ist zu beachten, dass sich bei der Messung der Diskriminierung sowohl Über- als auch Unterschätzungen ergeben können. Eine Überschätzung resultiert dann, wenn die produktivitätsbezogenen Merkmale nicht vollständig erfasst werden und die Gruppe mit den geringeren Einkommen eine unvorteilhaftere Ausstattung an nicht-beobachteten Merkmalen aufweist. Weist diese Gruppe hingegen eine vorteilhaftere Ausstattung an nicht-beobachteten Merkmalen auf, dann ergibt sich eine Unterschätzung der Diskriminierung.

Zweitens ist das hier präsentierte Modell zur Analyse der Diskriminierung in der Literatur in verschiedener Hinsicht erweitert worden. Erstens könnte durch die Berücksichtigung von Interaktionseffekten gemessen werden, wie sich das Einkommen der Ausländer ändern würde, wenn sie für den Ausstattungsunterschied auch die Differenz ihrer Koeffizienten erhalten würden [JONES/KELLEY (1984)]. Zweitens liessen sich die Gl. (10.9) bzw. (10.10) durch die Berücksichtigung von Gewichtungsfaktoren verallgemeinern. Über eine Matrix W liesse sich bestimmen, wie die Unterschiede zwischen den Koeffizienten des Diskriminierungseffektes bzw. zwischen den Mittelwerten des Ausstattungseffektes gewichtet werden

249 Siehe BERNDT (1996). 
könnten. ${ }^{250}$ Drittens könnten zur Messung und Analyse der Einkommensdiskriminierung nicht nur die mittleren Einkommensdifferentiale, sondern die gesamte Verteilung der Einkommensdiskriminierung berücksichtigt werden. ${ }^{251}$

\subsubsection{EMPIRISCHE ERGEBNISSE}

In den TAB. 10.11 und 10.12 finden sich die empirischen Ergebnisse zur Einkommenszerlegung. In beiden Tabellen werden sowohl die Ergebnisse der Zerlegung aus Gl. (10.9) als auch aus Gl. (10.10) ausgewiesen. Aus der Einkommenszerlegung für die in- und ausländischen Männer in TAB. 10.11 lassen sich drei wesentliche Erkenntnisse ableiten. Erstens zeigt sich, dass sowohl der Ausstattungs- als auch der Diskriminierungseffekt zugunsten der Inländer ausfällt, wobei der Diskriminierungseffekt im Vergleich zum Ausstattungseffekt einen wesentlich grösseren Erklärungswert besitzt.

Tab. 10.11: Einkommenszerlegung Männer (Inländer-Ausländer)

\begin{tabular}{|l|cc|cc|c|}
\hline Variablen & \multicolumn{2}{|c|}{$\begin{array}{c}\text { Ausstattungseffekt } \\
(10.9)\end{array}$} & $\begin{array}{c}\text { Diskriminierungseffekt } \\
(10.10)\end{array}$ & $\begin{array}{c}\text { Einkommens- } \\
\text { differenz }\end{array}$ \\
\hline CONSTANT & & & -0.047 & -0.047 & -0.047 \\
SCH & 0.034 & 0.026 & 0.134 & 0.142 & 0.168 \\
EXPE & -0.034 & -0.016 & 0.137 & 0.120 & 0.104 \\
EMWM & 0.012 & 0.011 & 0.003 & 0.005 & 0.016 \\
EMBM & 0.018 & 0.021 & -0.005 & -0.008 & 0.013 \\
SECTOR3 & 0.018 & 0.006 & 0.017 & 0.029 & 0.035 \\
UNPL & 0.002 & 0.003 & 0.007 & 0.006 & 0.010 \\
NTHEU_IC & & & -0.062 & -0.062 & -0.062 \\
STHEU & & & -0.044 & -0.044 & -0.044 \\
YSM & & & -0.037 & -0.037 & -0.037 \\
\hline Gesamteffekte & $\mathbf{0 . 0 5 1}$ & $\mathbf{0 . 0 5 2}$ & $\mathbf{0 . 1 0 4}$ & $\mathbf{0 . 1 0 4}$ & $\mathbf{0 . 1 5 5}$ \\
\hline
\end{tabular}

Quelle: SAKE (1995), eigene Berechnungen

Zweitens geht aus TAB. 10.11 hervor, dass das Einkommensdifferential zwischen in- und ausländischen Männern vor allem durch die Ausbildungs- und die Berufs-

250 Siehe Blinder (1973), OAXaCA (1973), ReImers (1983) und Velling (1995a). Die Vernachlässigung der Gewichtungsmatrix wurde in der Literatur verschiedentlich kritisiert [siehe z.B. COTTON (1988) und NEUMARK (1988)].

251 Siehe JENKINS (1994). JENKINS (1994: 82) argumentiert in diesem Zusammenhang, dass das im Rahmen der OAXACA/BLINDER-Zerlegung ermittelte Ausmass der Diskriminierung "... may be consistent with very different distributions of discrimination experience". 
erfahrungsvariable bedingt ist. Wie zu sehen ist, spielt der Diskriminierungseffekt eine wesentlich wichtigere Rolle als der Ausstattungseffekt, d.h. Ausländer erzielen auf ihrer Ausbildung bzw. Berufserfahrung geringere Ertragsraten als die Inländer. Eine Erklärung hierfür besteht in der unvollständigen Transferierbarkeit der im Ausland erworbenen Ausbildung bzw. Berufserfahrung.

Drittens zeigt der Blick auf die Effekte der ausländerspezifischen Variablen, dass diese zu einer Verringerung des Diskriminierungseffektes beitragen, da alle drei Effekte ein negatives Vorzeichen besitzen. Wie bereits erwähnt wurde, hängt das Vorzeichen der beiden Dummy-Variablen der Nationalitätengruppen allerdings von der Wahl der Referenzgruppe ab. Wären anstelle der Nicht-Europäer die Nordeuropäer als Basiskategorie verwendet worden, wären die Vorzeichen der beiden Dummy-Variablen positiv ausgefallen.

Tab. 10.12: Einkommenszerlegung Frauen (Inländer-Ausländer)

\begin{tabular}{|l|cc|cc|c|}
\hline Variablen & \multicolumn{3}{|c|}{$\begin{array}{c}\text { Ausstattungseffekt } \\
(10.9)\end{array}$} & $\begin{array}{c}\text { Diskriminierungseffekt } \\
(10.10)\end{array}$ & $\begin{array}{c}\text { Einkommens- } \\
\text { differenz }\end{array}$ \\
\hline CONSTANT & & & 0.084 & 0.084 & 0.084 \\
SCH & 0.022 & 0.017 & 0.114 & 0.118 & 0.136 \\
EXPE & -0.021 & -0.017 & 0.030 & 0.026 & 0.009 \\
EMWM & 0.006 & 0.013 & -0.016 & -0.023 & -0.010 \\
EMBM & 0.003 & 0.002 & 0.002 & 0.003 & 0.005 \\
SECTOR3 & 0.016 & 0.018 & -0.008 & -0.010 & 0.008 \\
UNPL & -0.002 & -0.008 & -0.053 & -0.047 & -0.055 \\
NTHEU_IC & & & -0.044 & -0.044 & -0.044 \\
STHEU & & & -0.018 & -0.018 & -0.018 \\
YSM & & & -0.016 & -0.016 & -0.016 \\
LAMBDA & -0.002 & -0.002 & & & -0.002 \\
\hline Gesamteffekte & $\mathbf{0 . 0 2 2}$ & $\mathbf{0 . 0 2 3}$ & $\mathbf{0 . 1 1 0}$ & $\mathbf{0 . 1 0 9}$ & $\mathbf{0 . 1 3 2}$ \\
\hline
\end{tabular}

Quelle: SAKE (1995), eigene Berechnungen

In TAB. 10.12 finden sich die Ergebnisse zur Einkommenszerlegung zwischen inund ausländischen Frauen. Der Vergleich mit TAB. 10.11 zeigt, dass das Einkommensdifferential bei den Männern rund 17 Prozent grösser ist als bei den Frauen. Wie aus TAB. 10.12 hervorgeht, besitzt der Diskriminierungseffekt bei den Frauen jedoch eine wesentlich grössere Bedeutung zur Erklärung des Einkommensdifferentials als bei den Männern. Während bei den Männern rund ein Drittel des Einkommensdifferentials durch den Ausstattungseffekt erklärt wird, liegt der Anteil des Ausstattungseffektes bei den Frauen bei lediglich rund 17 Prozent. 
Im Gegensatz zu den Männern ist bei den Frauen lediglich die Ausbildung ein relevanter Faktor bei der Erklärung des EInkommensdifferentials, wobei auch hier der Diskriminierungseffekt dominiert. Mit den Ergebnissen in TAB. 10.11 in Einklang stehen ebenfalls die Effekte der ausländerspezifischen Variablen, die auch bei den Frauen zu einer Verminderung des Diskriminierungseffektes beitragen. Insgesamt zeigt der Vergleich der Ergebnisse zwischen Männern und Frauen, dass die Effekte bei den Frauen weniger eindeutig ausfallen als bei den Männern. Abschliessend ist zu erwähnen, dass von der Selektionskorrekturvariablen ein geringfügig positiver Ausstattungseffekt zugunsten der Ausländerinnen ausgeht.

\subsection{FAZIT}

Aus den Ausführungen zur Einkommensperformance in diesem Kapitel lassen sich drei zentrale Schlussfolgerungen ableiten. Erstens bestätigen die empirischen Ergebnisse zur Einkommensperformance die Hypothese, dass zwischen der Beschäftigungs- und der Einkommensperformance ein enger Zusammenhang besteht. Einerseits dient die Humankapitalausstattung als zentrale Determinante sowohl der Beschäftigungs- als auch der Einkommensperformance. Andererseits wird die Einkommensperformance aber auch sehr stark von der beruflichen Stellung sowie den sektoralen Beschäftigungsanteilen der Wirtschaftssubjekte beeinflusst. Diese Ergebnisse verdeutlichen somit, dass die Einkommensperformance im wesentlichen durch die Beschäftigungsperformance determiniert ist.

Zweitens haben die deskriptive und empirische Analyse gezeigt, dass ähnlich wie Falle der Beschäftigungsperformance substantielle geschlechts- und nationalitätenspezifische Unterschiede bestehen. Die empirischen Ergebnisse lassen erkennen, dass Männer sowohl bei den In- als auch bei den Ausländern deutlich höhere Einkommen erzielen als Frauen. Dieses Ergebnis ist u.a. auf die oben genannten Unterschiede in den individuellen Charakteristika, aber auch auf Divergenzen in den sektoralen Beschäftigungsanteilen und der beruflichen Stellung zurückzuführen. Mit Blick auf die verschiedenen Nationalitätengruppen lässt sich festhalten, dass Nordeuropäer die vergleichsweise beste Performance aufweisen. Gemäss den Ergebnissen der deskriptiven Analyse übertreffen sie sowohl bei den Männern als auch bei den Frauen die Inländer.

Drittens geht aus der Zerlegung der Einkommensdifferentiale in einen Ausstattungs- und einen Diskriminierungseffekt hervor, dass der Diskriminierungseffekt sowohl bei den Männern als auch bei den Frauen einen wesentlich grösseren Stellenwert zur Erklärung der Einkommensdifferentiale besitzt als der Ausstattungseffekt. Gleichzeitig zeigt sich, dass bei den Männern die Ausbildung und die Berufserfahrung, bei den Frauen lediglich die Ausbildung, die grösste Bedeutung zur Erklärung des Einkommensdifferentials besitzen. Die ausländerspezifischen Variablen reduzieren schliesslich aufgrund der negativen Vorzeichen bei Frauen wie auch bei Männern den Diskriminierungseffekt. 


\section{SCHLUSSFOLGERUNGEN UND AUSBLICK}

Die theoretischen und empirischen Ausführungen der vorliegenden Studie haben die Zielsetzung verfolgt, einen wissenschaftlichen Beitrag zum besseren Verständnis des Zusammenhangs zwischen den beiden Themenkomplexen Migration und Arbeitsmarkt zu leisten. Erstmalig wurde hierzu die Performance von Einwanderern auf dem schweizerischen Arbeitsmarkt untersucht. Die empirischen Ergebnisse dienen dabei nicht nur der Erweiterung des Informationsstandes, sondern helfen auch bei der Planung und Evaluation von Politikinstrumenten im $\mathrm{Be}$ reich der Ausländer- und Migrationspolitik.

Im folgenden werden in einem ersten Schritt die wichtigsten theoretischen und empirischen Ergebnisse dieser Studie zusammengefasst. Darauf aufbauend sowie unter Berücksichtigung der Ausführungen aus dem Überblicksteil werden in einem zweiten Schritt einige wirtschaftspolitische Schlussfolgerungen zur Ausbzw. Umgestaltung der schweizerischen Migrationspolitik abgeleitet. In einem dritten Schritt folgen schliesslich im Rahmen eines Forschungsausblicks einige Überlegungen zu ergänzenden Forschungsthemen zur Arbeitsmarktperformance, die im Rahmen der vorliegenden Studie nicht berücksichtigt werden konnten.

\subsection{ZENTRALE ERGEBNISSE}

\subsubsection{ERGEBNISSE DER THEORETISCHEN ANALYSE}

Zwei zentrale Ergebnisse lassen sich aus der theoretischen Analyse dieser Studie ableiten. Erstens haben die theoretischen Ausführungen zur Arbeitsmarktperformance in Kapitel 5 gezeigt, dass die Humankapitaltheorie einen wichtigen Beitrag zur Erklärung der Arbeitsmarktperformance von Einwanderern zu leisten vermag, da sie Hinweise auf die zu erwartenden sozio-ökonomischen Profile der Einwanderer gibt. Aufgrund des Investitionscharakters ist zumindest für Arbeitsmigranten $\mathrm{zu}$ erwarten, dass vor allem für junge und gutausgebildete Arbeitskräfte Migrationsanreize bestehen. Wie die theoretischen Überlegungen gezeigt haben, wird die Performance der Zuwanderer von mehreren Faktoren bestimmt. Erstens besitzen die im Herkunftsland erworbenen Fähigkeiten und Qualifikationen wesentlichen Einfluss auf die Performance. Zweitens spielt die internationale Transferierbarkeit des Humankapitals der Einwanderer eine zentrale Rolle. Drittens ist der Umfang der von den Zuwanderern im Zielland getätigten landesspezifischen $\mathrm{Hu}$ mankapitalinvestitionen wichtig. Viertens beeinflussen verschiedene intervenierende Faktoren, wie die Nachfrage nach Einwanderern oder die rechtlichen Rahmenbedingungenen der Migrationspolitik, die Performance ebenfalls. 
Zweitens haben die theoretischen Überlegungen in Kapitel 6 aber auch gezeigt, dass die Arbeitsmarktperformance nicht nur von produktivitätsbezogenen Faktoren wie der Humankapitalausstattung beeinflusst wird, sondern dass auch diskriminierungsbedingte Faktoren für die Erklärung der Arbeitsmarktperformance von Bedeutung sein können. Es kann sich dabei einerseits um institutionelle Faktoren handeln, wie rechtliche Rahmenbedingungen der Migrationspolitik. Andererseits besteht die Möglichkeit, dass Unterschiede in der Beschäftigungsperformance auf einer unfreiwilligen asymmetrischen beruflichen und sektoriellen Allokation der in- und ausländischen Arbeitskräfte beruhen, so dass von einer Segmentation des Arbeitsmarktes gesprochen werden kann. Drittens ist denkbar, dass nicht-produktivitätsbedingte Einkommensdifferentiale zwischen In- und Ausländern auf Einkommensdiskriminierung zurückzuführen sind.

\subsubsection{ERGEBNISSE DER EMPIRISCHEN ANALYSE}

Die Resultate der theoretischen Analyse bilden den Ausgangspunkt der empirischen Analyse, aus der sich ebenfalls drei zentrale Erkenntnisse ableiten lassen. Erstens zeigen die empirischen Ergebnisse, dass zwischen In- und Ausländern signifikante Unterschiede in den sozio-demographischen und sozio-ökonomischen Profilen bestehen. Diese Unterschiede manifestieren sich vor allem in der Qualifikationsstruktur, die für Inländer aufgrund der höheren Anteile in den oberen Ausbildungssegmenten deutlich vorteilhafter ausfällt als für Ausländer. Da die sozioökonomischen Profile eine wesentliche Determinante der Beschäftigungs- und Einkommensperformance darstellen, erstaunt es nicht, dass zwischen In- und Ausländern diesbezüglich ebenfalls substantielle Divergenzen bestehen. Diese äussern sich i.w.S. in Unterschieden in der beruflichen Stellung sowie den sektoralen Beschäftigungsanteilen und i.e.S. in Unterschieden in den Erwerbsquoten, den Erwerbslosenquoten sowie den Einkommensniveaus.

Während bei den inländischen Männern und Frauen die Anteile an selbständig Erwerbstätigen bzw. Arbeitnehmern mit Vorgesetztenfunktion bei rund 55 bzw. 33 Prozent liegen, fallen diese Anteile bei den Ausländern mit rund 40 bzw. 20 Prozent deutlich geringer aus. Desweiteren sind Inländer weit stärker in den höherqualifizierten Segmenten des tertiären Sektors (Banken-, Versicherungs- und Immobiliensektor) vertreten als Ausländer, die vor allem in der Verarbeitenden Produktion, im Baugewerbe sowie im Handel und Gastgewerbe tätig sind. Diese Unterschiede in der beruflichen Stellung bzw. den sektoralen Beschäftigungsanteilen, die auf eine partielle Segmentation des Arbeitsmarktes hinweisen, können als Ursache für die deutlich höheren Erwerbslosenquoten sowie die tieferen Einkommen der Ausländer (im Vergleich zu den Inländern) angesehen werden. Wie aus der Einkommenszerlegung im Rahmen der Analyse der Einkommensdiskriminierung hervorgeht, sind die Einkommensunterschiede zwischen In- und Ausländern zu einem substantiellen Teil durch Einkommensdiskriminierung gegen- 
über den Ausländern bedingt. So ist das Einkommensdifferential von 15.5 Prozent bei den Männern bzw. 13.2 Prozent bei den Frauen zu zwei Dritteln bzw. mehr als vier Fünfteln auf Diskriminierung zurückzuführen.

Zweitens zeigt die empirische Analyse aber auch, dass nicht nur zwischen In- und Ausländern, sondern auch zwischen Männern und Frauen deutliche Unterschiede in der Beschäftigungs- und Einkommensperformance bestehen. Augenfällig ist vor allem, dass Frauen ein deutlich schlechteres sozio-ökonomisches Profil aufweisen als Männer. Es zeigt sich, dass der Anteil der Frauen in den höchsten Ausbildungssegmenten bei allen Nationalitätengruppen z.T. deutlich unter jenem der Männer liegt. Dieses Ergebnis ist u.a. auf geschlechtsspezifische Divergenzen in den Erwerbstätigkeitsprofilen über den Lebenszyklus zurückzuführen. Analog zu den vorangegangenen Ausführungen beeinflussen diese Ausstattungsunterschiede die Beschäftigungs- und Einkommensperformance, so dass Frauen erwartungsgemäss deutlich höhere Beschäftigungsanteile in den unteren Hierarchieebenen aufweisen. Gleichzeitig sind die weiblichen Erwerbstätigen stärker als die männlichen im Handel und Gastgewerbe sowie in den Branchen des sonstigen Dienstleistungssegments beschäftigt.

Mit Blick auf die Arbeitsmarktperformance i.e.S. fällt vor allem auf, dass Frauen deutlich geringere Erwerbsquoten besitzen als Männer und dass sie in viel stärkerem Mass teilzeiterwerbstätig sind, wobei dieses Ergebnis vor allem für die inländischen Frauen Gültigkeit besitzt. Diese Resultate implizieren, dass die traditionelle Rollenverteilung zwischen Männern und Frauen in der Schweiz immer noch relativ ausgeprägt ist. Hinsichtlich der Erwerbslosenquote bestehen ebenfalls deutliche geschlechtsspezifische Unterschiede, wobei die Erwerbslosenquoten der Frauen, mit Ausnahme der Nordeuropäer, deutlich über jenen der Männer liegen. Weniger eindeutig fallen die Aussagen zur Erwerbslosigkeitsdauer aus. Während der Anteil der Personen mit einer Erwerbslosigkeitsdauer von mehr als zwei Jahren bei den Frauen wesentlich höher ist als bei den Männern, trifft das Gegenteil für Personen mit einer Erwerbslosigkeitsdauer zwischen 12 und 24 Monaten zu. Schliesslich weisen Männer im Vergleich zu Frauen auch eine deutlich vorteilhaftere Einkommensperformance auf. Während bei den in- und ausländischen Männern der Anteil der Personen mit einem jährlichen Bruttoerwerbseinkommen von mindestens $78^{\prime} 000 \mathrm{CHF}$ bei 45 bzw. rund 25 Prozent liegt, sind lediglich rund 9 Prozent der Inländerinnen bzw. 16.5 Prozent der Ausländerinnen in diesen Einkommenssegmenten vertreten.

Drittens zeigt die empirische Analyse schliesslich, dass zwischen den Ausländern verschiedener Nationalitätengruppen substantielle Performanceunterschiede bestehen. Die Unterscheidung von drei Nationalitätengruppen (Nordeuropäer, Südeuropäer, Nicht-Europäer) impliziert daher, dass die bisherigen Aussagen zur Arbeitsmarktperformance der Ausländer relativiert werden müssen. Die Differenzierung der Analyse nach Nationalitätengruppen führt erwartungsgemäss zum Ergeb- 
nis, dass die Zuwanderer aus Nordeuropa die besten Ausbildungsprofile besitzen, gefolgt von den Nicht-Europäern und den Südeuropäern. Die Ausländer aus nichteuropäischen Staaten weisen sowohl in den höchsten als auch in tiefsten Ausbildungssegmenten relativ hohe Anteile auf, was im wesentlichen auf das hohe Aggregationsniveau innerhalb dieser Nationalitätengruppe zurückzuführen ist.

Hinsichtlich der Beschäftigungs- und der Einkommensperformance zeigt sich, dass die Nordeuropäer nicht nur im Vergleich zu den anderen Nationalitätengruppen, sondern auch im Vergleich zu den Inländern die höchsten beruflichen Stellungen erreichen und die vorteilhaftesten sektoralen Beschäftigungsanteile aufweisen und damit sowohl in Bezug auf die Erwerbsquoten bzw. Erwerbslosenquoten als auch in Bezug auf die Einkommenshöhe am erfolgreichsten sind. Diese Aussage besitzt vor allem für Männer, in weiten Teilen aber auch für Frauen Gültigkeit. Es ist somit klar ersichtlich, dass zur Analyse der Arbeitsmarktperformance eine differenzierte Vorgehensweise erforderlich ist, da andernfalls keine aussagekräftigen Ergebnisse gewährleistet werden können.

Schliesslich wurde im Rahmen der deskriptiven Analyse auch der Einfluss kohortenspezifischer Effekte auf die Arbeitsmarktperformance berücksichtigt. Es zeigt sich, dass zwischen den verschiedenen Einwanderungskohorten zwar signifikante Unterschiede bestehen, dass sich daraus aber, im Gegensatz zu den bisherigen Ergebnissen, keine eindeutigen Aussagen ableiten lassen. Dies ist u.a. darauf zurückzuführen, dass die Zusammensetzung der Einwanderungskohorten stark von der Zuwanderungsstruktur abhängt. Es ist allerdings darauf hinzuweisen, dass in der ökonometrischen Analyse Kohorteneffekte nicht berücksichtigt werden konnten, da diese auf Querschnittsdaten beruht.

\subsection{WIRTSCHAFTSPOLITISCHE SCHLUSSFOLGERUNGEN}

Im Rahmen der wirtschaftspolitischen Schlussfolgerungen dieses Abschnitts steht nochmals die schweizerische Migrationspolitik im Zentrum des Interesses, da sich aufgrund der theoretischen und empirischen Ergebnisse dieser Studie die Frage nach Reformvorschlägen für die schweizerische Migrationspolitik stellt. Wie GOLDER (1997b) im Rahmen eines internationalen Vergleichs gezeigt hat, ist eine nationalitätenspezifische Zulassungspolitik, wie sie im Rahmen der Drei-KreisePolitik praktiziert wird, grundsätzlich nicht ungeeignet, eine positive Selektion der Einwanderer herbeizuführen und damit eine gute Arbeitsmarktperformance der ausländischen Arbeitskräfte zu gewährleisten. Es gilt in diesem Zusammenhang allerdings zwei wichtige Einwände zu bedenken. Erstens besitzt die Definition bzw. Ausgestaltung der einzelnen Kreise einen grossen Einfluss auf die Arbeitsmarktperformance der Einwanderer. Die Bedeutung dieses Zusammenhangs lässt sich anhand der Performanceunterschiede zwischen den verschiedenen Nationalitätengruppen veranschaulichen. 
Zweitens ist zu berücksichtigen, dass eine Migrationspolitik, die auf der Basis geographisch-politischer Räume verschiedene Stufen der Einwanderungsberechtigung definiert, dem Vorwurf der Diskriminierung ausgesetzt ist. Diskriminierung bedeutet in diesem Fall, dass Zuwanderung aufgrund von personenspezifischen Merkmalen, im speziellen aufgrund der Staatsangehörigkeit, beschränkt wird. Es muss in diesem Zusammenhang erwähnt werden, dass sowohl in den Vereinigten Staaten als auch in Kanada der öffentliche Druck gegen die Diskriminierung der bestehenden nationalitätenspezifischen Einwanderungspolitik Mitte der 60er Jahre mit zur Neuorientierung der Migrationspolitiken in diesen beiden Ländern beigetragen hat. Die Drei-Kreise-Politik steht auch in der Schweiz in der Kritik, da insbesondere die Kommission gegen Rassismus zum Schluss gekommen ist, dass die Ausländerpolitik im Rahmen der Drei-Kreise-Politik einen Verstoss gegen das in der Schweiz geltende Diskriminierungsverbot darstellt.

Welche Reformvorschläge lassen sich unter Berücksichtigung dieser Sachverhalte somit für die schweizerische Migrationspolitik ableiten? Ein möglicher Reformvorschlag bestünde in der Flankierung der nationalitätenspezifischen Zulassungspolitik der Drei-Kreise-Politik durch ein Punktesystem. Durch diese Vorgehensweise würden sich jedoch zwei Probleme nicht lösen lassen. Erstens würde diese Reform im Widerspruch zur angestrebten Zielsetzung einer vollständigen Freizügigkeit zwischen der Schweiz und den Mitgliedstaaten des Europäischen Wirtschaftsraumes stehen, da der freie Personenverkehr keine Zulassungsbeschränkungen gestatten würde. Zweitens bliebe das Diskriminierungsproblem aufgrund der nationalitätenspezifischen Zulassungsbeschränkungen weiterhin bestehen.

Im folgenden wird daher eine politisch durchführbare und ökonomisch sinnvolle Reformalternative vorgestellt, die den angesprochenen Problemen Rechnung trägt und die schweizerische Migrationspolitik auf ein langfristig tragfähiges Fundament stellt. Dieser Reformvorschlag, der weitgehend auf GoLDER (1997b) beruht, hat bereits Eingang in die politische Diskussion gefunden, da die Expertenkommission für die Migrationspolitik im Herbst des vergangenen Jahres ebenfalls Reformvorschläge vorgestellt hat, die sich in weiten Teilen mit den hier präsentierten Vorschlägen decken (siehe auch Abschnitt 3.4). Die vorliegende Studie leistet somit, in Übereinstimmung mit der in der Einleitung formulierten Zielsetzung, einen Beitrag zur wissenschaftlichen Fundierung der Reformvorschläge der Expertenkommission.

Bei diesem Reformvorschlag handelt es sich um eine sog. Zwei-Kreise-Politik. Im ersten Kreis wird dabei, analog zur bisherigen Politik, die vollständige Freizügigkeit der Arbeitskräfte zwischen der Schweiz und den Mitgliedstaaten des Europäischen Wirtschaftsraums angestrebt. Im zweiten Kreis, in dem sich die restlichen Staaten befinden, sollte zur Steuerung der Zuwanderung ein Punktesystem zur Anwendung gelangen. An die Stelle des selektiven, nationalitätenspezifischen $\mathrm{Zu}$ gangs ausländischer Arbeitskräfte innerhalb des bisherigen zweiten Kreises sowie 
der diskriminierenden Zugangsverweigerung für Arbeitskräfte des dritten Kreises sollte ein Punktesystem treten, das den sozio-ökonomischen Charakteristika der Zuwanderer Rechnung trägt.

Aus dieser Neuorientierung der schweizerischen Migrationspolitik ergeben sich vor allem zwei Vorteile. Zum einen ermöglicht ein Punktesystem im Gegensatz zu einer nationalitätenspezifischen Zulassungspolitik die direkte Berücksichtigung der individuellen sozio-ökonomischen und sozio-demographischen Profile der Einwanderer bei der Zulassungsentscheidung. Dadurch kann den Bedürfnissen des Arbeitsmarktes in verstärktem Masse Rechnung getragen werden. Zum anderen findet mit dem Übergang von der nationalitätenspezifischen Zulassungspolitik zum Punktesystem auch eine Abkehr von der diskriminierenden Behandlung potentieller Einwanderer statt, indem Einwanderungsberechtigungen nicht mehr aufgrund der Staatsangehörigkeit erteilt werden, sondern aufgrund der spezifischen individuellen Charakteristika der einzelnen Wirtschaftssubjekte.

Bei der Implementation des Punktesystems sind einige zentrale Aspekte zu berücksichtigen. Erstens sollte sich die Vergabe der Punkte, nach dem bewährten Beispiel Kanadas und Australiens, an folgenden Kriterien orientieren: Alter, Familienstand, Schul- und Berufsausbildung, Berufserfahrung, Sprachkenntnisse, die Bereitschaft zu finanziellen Investitionen sowie zur Schaffung von Arbeitsplätzen, bereits vorliegende Stellenzusage sowie Knappheitsindikatoren nach Branchen und Berufsgruppen. ${ }^{252}$ Analog zur kanadischen Praxis wäre es sinnvoll, Investoren und Unternehmer im Rahmen des Punktesystems bevorzugt zu behandeln.

Zweitens lässt sich im Rahmen eines Quotensystems, wie es in der Schweiz bereits praktiziert wird, eine flexible, an den Bedürfnissen des Arbeitsmarktes ausgerichtete Steuerung der Zuwanderung verwirklichen. Wie die schweizerischen Erfahrungen jedoch gezeigt haben, insbesondere das Scheitern der Rotationspolitik, sollte die Migrationspolitik nicht in den Dienst der Konjunkturpolitik gestellt werden, sondern sich an längerfristigen strukturellen und gesamtwirtschaftlichen Entwicklungen orientieren. Ähnlich wie in Kanada sollte von der Regierung für einen mittelfristigen Zeithorizont eine Zielgrösse festgelegt werden, die jährlich unter Berücksichtigung der gesamtwirtschaftlichen Lage überprüft werden sollte.

Drittens sollte mit dem Übergang zum Punktesystem auch eine Vereinfachung der Aufenthaltskategorien stattfinden, indem klar zwischen temporärer und permanenter Einwanderung unterschieden wird. Im Falle temporärer Zuwanderung, die in erster Linie dem Ziel der Aus- und Weiterbildung sowie dem Kaderaustausch innerhalb multinationaler Unternehmen dienen sollte, müsste die Aufenthaltsdauer klar begrenzt werden. Zudem dürfen sich aus dem zeitlich beschränkten Aufenthalt keine Rechte für eine Verlängerung der Aufenthaltserlaubnis ableiten lassen. Der Familiennachzug sollte demgegenüber vollständig liberalisiert werden. Im

252 Siehe ZIMMERMANN (1997) und VELLING/WOYDT (1993). 
Falle permanenter Einwanderung sollten alle Beschränkungen hinsichtlich der geographischen und beruflichen Mobilität sowohl für die Zuwanderer als auch für deren Familienangehörige abgebaut werden. Durch diese Massnahmen würde insbesondere das umstrittene Saisonnierstatut wegfallen.

Neben diesen Bestimmungen, welche die Zulassungsbedingungen der Zuwanderung regeln, ist aber in verstärktem Masse auch eine aktive Integrationspolitik zu verfolgen. Die Notwendigkeit hierzu ergibt sich aufgrund der massgeblichen Verlagerung der Migrationsmotive in den vergangenen Jahrzehnten, die zu einer markanten Erhöhung des Anteils der Netzwerk-, Familien- und Flüchtlingsmigration geführt haben. $\mathrm{Da}$ sich diese Wanderungsbewegungen aufgrund humanitärer Überlegungen weder effektiv steuern noch reglementieren lassen, stellt lediglich die Arbeitsmigration einen durch die Migrationspolitik beeinflussbaren Politikparameter dar. Damit alle Einwanderer eine Chance haben, ihr Fähigkeitspotential zu entfalten und damit auch in der Lage wären, einen Beitrag zur Prosperität der schweizerischen Volkswirtschaft zu leisten, muss den Einwanderern die Eingliederung in die schweizerische Gesellschaft erleichtert bzw. ermöglicht werden. Diese Überlegungen unterstreichen daher auch den flankierenden Reformbedarf im Bereich der Asylpolitik. Erstens muss die Schweiz weiterhin ihren völkerrechtlichen Verpflichtungen nachkommen. Zweitens sind gegenüber den anerkannten Flüchtlingen, die eine permanente Aufenthaltsberechtigung in der Schweiz besitzen, die Integrationsbemühungen zu verstärken. Drittens ist, wie im Falle temporärer Einwandererung, für vorläufig aufgenommene Flüchtlinge eine Liberalisierung der Erwerbstätigkeit zu fordern, ohne dass sich dadurch jedoch Rechte für eine Verfestigung des Aufenthaltsstatus ableiten lassen dürften.

Die angestrebte vollständige Freizügigkeit im Güter- und Personenverkehr zwischen der Schweiz und den Mitgliedstaaten des Europäischen Wirtschaftsraums innerhalb des ersten Kreises wie auch die Arbeitskräfteselektion im Rahmen des Punktesystems des zweiten Kreises sind grundsätzlich positiv zu bewerten. Insbesondere im Hinblick auf den Europäischen Wirtschaftsraum eröffnen diese Massnahmen der Schweiz bessere Wettbewerbsbedingungen sowie einen verbesserten Zugang zum europäischen Arbeitsmarkt. Aus ökonomischer Sicht erscheint es dabei am sinnvollsten, den Allokationsprozess auf dem europäischen Arbeitsmarkt den Marktkräften zu überlassen. Dies bedingt aber, dass sich die Marktkräfte ungehindert entfalten können müssten, da ansonsten mit Fehlallokationen zu rechnen wäre. Da dies aber für den Fall der Schweiz nicht zutrifft, müssen verstärkte flankierende Anstrengungen zur Liberalisierung und Deregulierung der Wirtschaftsstrukturen unternommen werden, um eine Allokation der Arbeitskräfte entsprechend den effektiven Knappheiten gewährleisten zu können.

Diese Forderung trifft in erster Linie für die stagnierenden und vom Wettbewerb abgeschotteten Branchen des binnenorientierten Sektors zu. Dies vor allem deshalb, weil Branchen wie das Gast- und das Baugewerbe aber auch die Verarbei- 
tende Produktion in den vergangenen Jahrzehnten durch ihre Nachfrage nach billigen ausländischen Arbeitskräften zu einer künstlichen Aufblähung dieser Branchen beigetragen haben. Dies hat dazu geführt, dass ein beträchtlicher Teil der ausländischen Erwerbstätigen ein unvorteilhaftes Qualifikationsprofil aufweist und daher nicht in den zukunftsorientierten und wettbewerbsfähigen Branchen eingesetzt werden kann. Eine Intensivierung des Wettbewerbs in diesen Branchen würde die Restrukturierung vorantreiben und damit verbunden auch den Bedarf an niedrig qualifizierten Arbeitskräften reduzieren.

Zusammenfassend lässt sich somit festhalten, dass für die schweizerische Migrationspolitik erheblicher Reformbedarf besteht, um die in der Einleitung skizzierten Herausforderungen der Zukunft erfolgreich bewältigen zu können. Wie diese Studie jedoch gezeigt hat, müssen diese Reformen von Deregulierungs- und Liberalisierungsmassnahmen, verstärkten Integrationsbemühungen sowie von Anpassungen im Bereich der Asylpolitik flankiert werden. Die zunehmende Globalisierung und Öffnung der Märkte sowie die verstärkte Internationalisierung der Wirtschaftsbeziehungen haben den Druck auf die einzelnen Volkswirtschaften erhöht, attraktive Standortfaktoren zu implementieren, um so im Wettbewerb der Standorte um die mobilen Faktoren Arbeit und Kapital konkurrenzfähig zu bleiben. Aus diesem Grund erscheint es unerlässlich, die Um- bzw. Restrukturierung der schweizerischen Volkswirtschaft hin zu einer Dienstleistungs- und Informationsgesellschaft voranzutreiben und die Flexibilität der schweizerischen Wirtschaft zu erhöhen. Gleichzeitig sind die Integrationsbemühungen zu verstärken, damit die Einwanderer ihre Fähigkeitspotentiale gewinnbringend in der schweizerischen Volkswirtschaft einsetzen und damit zur wirtschaftlichen Prosperität der Schweiz beitragen können.

\subsection{FORSCHUNGSAUSBLICK}

Abschliessend folgen nun im Rahmen eines Forschungsausblicks einige Überlegungen zu Vertiefungs- und Erweiterungsmöglichkeiten der Untersuchungen zur Arbeitsmarktperformance von Einwanderern, die jedoch im Rahmen der vorliegenden Studie nicht berücksichtigt werden konnten und Gegenstand zukünftiger Forschungsprojekte sein werden.

\subsubsection{VERTIEFUNGSMÖGLICHKEITEN DER ANALYSE}

Die empirische Analyse dieser Studie hat gezeigt, dass vor allem zwei nennenswerte Vertiefungsmöglichkeiten zur Analyse der Performance der Einwanderer existieren. Erstens wurde insbesondere im Zusammenhang mit der Analyse der intertemporalen Entwicklung der Arbeitsmarktperformance in Kapitel 8 auf die Bedeutung der Unterscheidung zwischen Querschnitts- und Paneldatenanalysen hingewiesen. Paneldaten besitzen gegenüber Querschnitts- und Längsschnittsdaten 
eine Reihe von Vorteilen. ${ }^{253}$ Zum einen beinhalten sie i.a. eine relativ grosse Anzahl an Datenpunkten, was zu einer Vergrösserung der Zahl der Freiheitsgrade und zu einer Verringerung der Kollinearität zwischen den erklärenden Variablen führt, so dass insgesamt eine Verbesserung der Effizienz der Schätzergebnisse erreicht werden kann.

Zum anderen, ermöglicht die Verwendung von Paneldaten aber vor allem die Analyse einer Reihe von Fragestellungen, die sich durch die Verwendung von Quer- und Längsschnittsdaten nicht beantworten lassen. Dies lässt sich am besten anhand von zwei Beispielen illustrieren. Einerseits erlaubt der Gebrauch von $\mathrm{Pa}$ neldaten eine korrekte Beobachtung dynamischer Entwicklungen. Dieser Aspekt ist insbesondere hinsichtlich der in Kapitel 8 angesprochenen Problematik der Analyse der Arbeitsmarktassimilierung von Bedeutung. Wie in ABB. 8.1 veranschaulicht wurde, kann die Vermischung von Alters- und Kohorteneffekten in Querschnittsbetrachtungen dazu führen, dass eine Überschätzung der effektiven Arbeitsmarktassimilierung stattfindet.

Andererseits kann auch die Verwendung mehrerer zeitlicher Folgen von Querschnittsdaten im Vergleich zu Paneldaten mit Problemen behaftet sein. Dieser Aspekt lässt sich anhand der Studie von BEN-PORATH (1973) veranschaulichen. Als Ausgangspunkt der Überlegungen diente die Annahme, dass in einem Querschnittsdatenset von verheirateten Frauen eine durchschnittliche jährliche Erwerbstätigkeitswahrscheinlichkeit von 50 Prozent besteht. Im einen Extremfall lässt sich dieses Ergebnis dahingehend interpretieren, dass es sich bei den verheirateten Frauen um eine homogene Bevölkerungsgruppe handelt, so dass jede Frau eine Erwerbstätigkeitswahrscheinlichkeit von 50 Prozent besitzt. Im anderen Extremfall einer heterogenen Bevölkerungsgruppe kann dieses Ergebnis aber auch bedeuten, dass 50 Prozent der Frauen immer erwerbstätig sind und 50 Prozent nie. Im ersten Fall kann somit davon ausgegangen werden, dass jede verheiratete Frau die Hälfte ihres Lebens erwerbstätig ist, während im zweiten Fall die eine Hälfte der Frauen immer erwerbstätig und die andere Hälfte nie erwerbstätig ist. Der zweite Fall impliziert, dass der gegenwärtige Arbeitsmarktstatus als perfekte Prognose des zukünftigen Arbeitsmarktstatus angesehen werden kann. Um zwischen diesen beiden Modellen differenzieren bzw. individuenspezifische Unterschiede aufdecken zu können, müssen Informationen über die individuellen Erwerbstätigkeitsprofile im Lebenszyklus zur Verfügung stehen. Dies ist aber nur dann möglich, wenn zu denselben Personen mehrere aufeinanderfolgende Beobachtungen vorliegen.

Oftmals lassen sich aber individuenspezifische Variablen nicht erheben bzw. wird das Verhalten der Untersuchungseinheiten von individuenspezifischen Faktoren beeinflusst, die nicht beobachtbar sind. Der zentrale Beitrag von Paneldatenmo-

253 Siehe Baltagi (1995), HSiao (1993), GREENE (1995) und RONNING (1991). 
dellen besteht darin, dass im Rahmen solcher Modelle versucht wird, diese individuenspezifischen Effekte zu modellieren, um der interindividuellen Heterogenität Rechnung tragen zu können. In der Literatur existieren vier verschiedene Basismodelle, auf welchen die Analyse von Paneldaten aufbaut. Diese Modelle unterscheiden sich hinsichtlich der Annahmen über die Koeffizienten und Konstanten, auf denen sie beruhen. Im ersten Modell wird unterstellt, dass die Steigungskoeffizienten konstant sind und lediglich die Achsenabschnitte zwischen den Individuen variieren. Das zweite basiert auf der Annahme, dass die Steigungskoeffizienten konstant sind und die Achsenabschnitte sowohl zwischen den Individuen als auch im Laufe der Zeit variieren. Im dritten Modell wird unterstellt, dass alle Koeffizienten zwischen den Individuen variieren. Im vierten wird schliesslich unterstellt, dass alle Koeffizienten sowohl zwischen den Individuen als auch über die Zeit variieren.

Modelle mit konstanten Steigungskoeffizienten und variablen Achsenabschnitten, d.h. die Modelle 1 und 2, werden in der Literatur am häufigsten für die Analyse von Paneldatenmodellen verwendet, da es sich bei ihnen um einfache und dennoch relativ allgemeine Alternativen zur Querschnittsanalyse handelt, in der unterstellt wird, dass alle Parameter für alle Untersuchungseinheiten dieselben Werte besitzen. Wird zur Vereinfachung von zeitspezifischen Effekten abstrahiert und lediglich von individuenspezifischen Effekten ausgegangen, lassen sich in Abhängigkeit der Annahmen bezüglich dieser Effekte zwei verschiedene Modelle unterscheiden. In der Literatur wird von sog. 'fixed effects models' gesprochen, wenn die individuenspezifischen Unterschiede als parametrische Verschiebungen der Regressionsfunktion interpretiert werden können, d.h die individuenspezifischen Effekte Konstanten sind. Diese Modelle lassen sich mit Hilfe der klassischen Schätzverfahren analysieren. Wird demgegenüber davon ausgegangen, dass die individuenspezifischen Unterschiede nicht konstant, sondern zufällig sind, dann wird von sog. 'random effects models' gesprochen. Zur Analyse dieser Modelle kann die 'zweistufige verallgemeinerte Methode der Kleinsten Quadrate' (twostage feasible GLS) verwendet werden.

Zweitens haben die theoretischen Überlegungen in Kapitel 7 gezeigt, dass mit signifikanten Performanceunterschieden zwischen den Einwanderern der Ersten und der Zweiten Generation zu rechnen ist. Diese Hypothese liegt vor allem darin begründet, dass Ausländer der Zweiten Generation i.a. über einen grösseren Bestand an ziellandesspezifischem Humankapital verfügen und ebenfalls besser im Zielland integriert sind. ${ }^{254}$ Nachdem im Rahmen der vorliegenden Studie lediglich die Arbeitsmarktperformance der Ausländer der Ersten Generation analysiert wurde, bestünde ein nächster Schritt im Vergleich der Arbeitsmarktperformance der Ersten und Zweiten Generation.

254 Siehe GANG/ZIMMERMANN (1996). 


\subsubsection{ERWEITERUNGSMÖGLICHKEITEN DER ANALYSE}

Während sich die vorangegangenen Ausführungen auf Ansätze zur Vertiefung der im Rahmen dieser Studie vorgestellten Ergebnisse bezogen haben, werden im folgenden zwei Vorschläge zur Erweiterung der Analyse präsentiert.

Erstens könnte die Analyse der Erwerbslosigkeitswahrscheinlichkeit durch dynamische Untersuchungen ergänzt werden. Diese Vorgehensweise würde einerseits eine umfassendere Analyse der Beschäftigungsperformance erlauben und andererseits den dynamischen Prozessen auf dem Arbeitsmarkt verstärkt Rechnung tragen. Zur Dynamisierung der Untersuchung der Erwerbslosigkeit bieten sich sog. Abgangsratenmodelle an. Als Analysegegenstand dient in diesen Modellen, unter der Annahme einer gegebenen Verweildauer im Ausgangszustand, die Wahrscheinlichkeit, von einem Ausgangszustand in einen Zielzustand überzugehen.

Im Standardmodell wird davon ausgegangen, dass lediglich zwei Zustände möglich sind - im vorliegenden Fall erwerbstätig oder erwerbslos - und dass lediglich ein Übergang vom Ausgangs- in den Zielzustand denkbar ist. Dieses einfache Modell lässt sich dadurch erweitern, dass mehrere Zielzustände betrachtet und multiple Übergänge zwischen den verschiedenen Zuständen berücksichtigt werden. So lässt sich z.B. der Abgang aus einer gegebenen Beschäftigung betrachten, wobei die Zielzustände entweder eine neue Beschäftigung oder Erwerbslosigkeit sein können. ${ }^{255}$

Diese Analyse ist für die Schweiz insbesondere deshalb von Interesse, weil als Folge des massiven Anstiegs der Erwerbslosenquoten die Befürchtung besteht, dass sich daraus ähnlich wie in den anderen OECD-Ländern eine persistente Sockelarbeitslosigkeit herausbilden wird. Von grossem Interesse ist somit die Frage, ob die Abgangsrate aus der Erwerbslosigkeit von der Dauer der Erwerbslosigkeit abhängig ist. Ein negativer Zusammenhang zwischen der Dauer und der Abgangswahrscheinlichkeit kann als Indiz für die zunehmende Persistenz der Erwerbslosigkeit gewertet werden.

Eine empirische Untersuchung dieser Fragestellung für die Schweiz findet sich in GERFIN (1996), der in seiner Analyse allerdings nur zwischen Männern und Frauen unterscheidet. ${ }^{256}$ GerFIN konnte zeigen, dass zwischen der Dauer der Erwerbslosigkeit und der Abgangswahrscheinlichkeit in weiten Bereichen ein positiver Zusammenhang besteht, so dass die Gefahr einer zunehmenden Persistenz nicht gegeben zu sein scheint. Dieses Ergebnis trifft allerdings nicht für Personen zu, die bereits über Erwerbslosigkeitserfahrungen verfügen. Für sie ist die Ab-

255 In der Literatur wird in diesem Zusammenhang auch von sog. 'Duration/Lifetime Data Models' und 'Proportional Hazard Models' gesprochen [siehe COX/OAKES (1984), GREENE (1995, 1996), KALBFLEISCH/PRENTICE (1980) sowie KIEFER (1988)].

Eine deskriptive Analyse dieses Zusammenhangs für Ausländer findet sich in BLATTNER/THEISS (1994). 
gangswahrscheinlichkeit zwar konstant, aber auch sehr klein, so dass die Wahrscheinlichkeit einer langfristigen Erwerbslosigkeit für diese Gruppe relativ gross ist. Mit Blick auf die empirischen Ergebnisse zur Beschäftigungs- und Einkommensperformance stellt sich insbesondere die Frage, ob hinsichtlich der Abgangsraten nationalitätenspezifische Unterschiede bestehen.

Zweitens ist hinsichtlich des Zusammenhangs zwischen Ausbildung und beruflicher Mobilität bei der Erklärung der Arbeitsmarktperformance mit einem Bedeutungszuwachs zu rechnen. Im Zentrum steht dabei die Frage nach der beruflichen Anpassungsfähigkeit der Wirtschaftssubjekte. Diese Fragestellung ist insbesondere mit Blick auf die grundlegenden strukturellen Veränderungen sowie die damit verbundenen Anpassungskosten von Bedeutung. Die 'Tertiarisierung' der schweizerischen Wirtschaft bedeutet, dass derzeit in allen Bereichen der Wirtschaft ein tiefgreifender Wandel der Produktionsstrukturen stattfindet, der durch eine "... innovationsorientierte Produktdifferenzierung und eine zunehmende Verflechtung und Vernetzung der verschiedenen Wirtschaftsbereiche gekennzeichnet ist" [Klodt/MaureR/SchimmelPFenNIG (1997: 212)]. Der Prozess der Tertiarisierung impliziert somit nicht nur die Verdrängung von Industriegütern durch Dienstleistungen und damit verbunden eine Verschiebung der sektoralen Produktions- und Beschäftigungsanteile, sondern vielmehr die Herausbildung neuer Produktionsstrukturen in allen Bereichen der Wirtschaft.

Diese Transformationsphase ist eng mit der Entwicklung moderner Informationsund Kommunikationstechnologien verknüpft, so dass Information zum zentralen Rohstoff der Zukunft avancieren wird. Die rasanten Entwicklungen im Bereich der Informationstechnologie implizieren nicht nur Veränderungen in den Produktions- sondern auch in den Unternehmens- und Marktstrukturen. Insbesondere die zunehmende Produktdifferenzierung und Globalisierung der Märkte erfordert eine verstärkte Flexibilisierung wirtschaftlichen Handelns. Gemäss KLODT/MAURER/SCHIMMELPFENNING impliziert der Übergang von der hierarchisch strukturierten Industriegesellschaft zur netzwerkorientierten Informationsgesellschaft, dass in Zukunft sowohl höhere als auch andere Qualifikationen der Arbeitnehmer gefragt sein werden.

Diese Strukturverschiebungen sowie die Trägheit des Arbeitsangebotes sind daher im wesentlichen als Ursachen der strukturellen Arbeitslosigkeit zu sehen. Um weitere Härten zu vermeiden, muss sichergestellt werden, dass der Übergang zur Informationsgesellschaft reibungslos verläuft. Hierzu sind verstärkte Liberalisierungs- und Deregulierungsmassnahmen notwendig. Desweiteren muss geklärt werden, ob der Staat den Übergangsprozess, aufgrund von Netzwerk- und Lockin-Effekten, durch Regulierungseingriffe erleichtern kann. Der wichtigste Beitrag zum Gelingen dieses Strukturwandels geht allerdings von der Reform des Bildungs- und Ausbildungssystems aus. Obschon sich das duale Berufsbildungssystem in der Schweiz in der Vermittlung praxisrelevanter Qualifikationen als leis- 
tungsfähig erweist, trifft dies nicht für die Anpassungsfähigkeit an veränderte Marktbedingungen zu. Schliesslich ist insbesondere der Hochschulbereich zu nennen, von dem aufgrund des nahezu kostenlosen Bildungsangebots gravierende Fehlanreize zum Erwerb nichtmarktgerechter Qualifikationen ausgehen. Die Analyse der Flexibilität auf dem Arbeitsmarkt, die sich u.a. in der beruflichen Mobilität spiegelt, sowie der Bedeutung von Aus- und Weiterbildung stellen somit zwei zentrale Untersuchungsgegenstände dar, die Aufschluss über die notwendigen Reformschritte geben können und damit einen Beitrag zur langfristigen Sicherung der Prosperität der schweizerischen Wirtschaft leisten können. 


\section{ANHANG 1: DESKRIPTIVE STATISTIKEN}

Tab. A8.2: Definition verschiedener Nationalitätengruppen

\begin{tabular}{|c|c|c|c|c|}
\hline \multicolumn{2}{|l|}{ Gastarbeiterländer } & \multicolumn{3}{|c|}{$\begin{array}{ll}\text { Aussereuropäische Staaten } & \text { (OTCT) }\end{array}$} \\
\hline Italien & $(I T A)$ & Osteuropa: & - Albanien & \\
\hline Spanien & $(E S P)$ & & - Bulgarien & \\
\hline Portugal & (POR) & & - Polen & \\
\hline Türkei & (TRK) & & $\begin{array}{l}\text { - Rumänien } \\
\text { - Tschechische }\end{array}$ & \\
\hline Ex-Jugoslawien & $(E X J)$ & & Republik & \\
\hline Europäische Industrieländer & & & - UdSSR & \\
\hline Deutschland & $(F R G)$ & & - Ungarn & \\
\hline Frankreich & (FRA) & Afrika: & - Algerien & \\
\hline Österreich & $(A U T)$ & & - Tunesien & \\
\hline Sonstige EU-/EFTA-Staaten & (OTEU) & & - Uganda & \\
\hline - Belgien & & Vorderasien: & - Ägypten & \\
\hline - Dänemark & & & - Iran & \\
\hline - Finnland & & & - Israel & \\
\hline - Griechenland & & & - Libanon & \\
\hline - Grossbritannien & & Hinterasien: & - Indien & \\
\hline - Irland & & & - Kambodscha & \\
\hline - Island & & & - Philippinen & \\
\hline - Liechtenstein & & & - Sri Lanka & \\
\hline - Luxemburg & & & - Tibet/China & \\
\hline - Niederlande & & & - Vietnam & \\
\hline - Norwegen & & Lateinamerika: & - Argentinien & \\
\hline - Schweden & & & - Brasilien & \\
\hline Sonstige Industriestaaten & (OTIC) & & - Chile & \\
\hline - Japan & & sonstige & & \\
\hline - Kanada & & Nationalitäten: & - andere & \\
\hline - USA & & & - Staatenlos & \\
\hline
\end{tabular}

Quelle: Eigene Darstellung gemäss Angaben der SAKE (1995)

Anmerkungen: Die Nationalitätengruppen sowie deren Abkürzungen sind jeweils kursiv und fett gedruckt 
Tab. A8.6: Einwanderungskohorten nach Nationalitätengruppen (in Pro-

\begin{tabular}{|c|c|c|c|c|c|c|c|}
\hline \multirow[t]{2}{*}{$\begin{array}{l}\text { Nationalitäten- } \\
\text { gruppen }\end{array}$} & \multicolumn{6}{|c|}{$\begin{array}{c}\text { Einwanderungskohorten } \\
\text { (Aufenthaltsdauer in Jahren) }\end{array}$} & \multirow[t]{2}{*}{$\begin{array}{c}\text { Ausländer } \\
\text { (Total) }\end{array}$} \\
\hline & $<1$ & $1-5$ & $5-10$ & $10-15$ & $15-20$ & $>20$ & \\
\hline \multicolumn{8}{|l|}{ Männer } \\
\hline Italien & 29.1 & 9.4 & 15.7 & 20.2 & 25.5 & 51.1 & 33.4 \\
\hline Spanien & & 0.2 & 5.8 & 6.9 & 18.3 & 7.9 & 7.5 \\
\hline Portugal & 10.2 & 7.2 & 12.1 & 20.7 & 12.9 & 0.5 & 7.9 \\
\hline Tïrkei & & 2.2 & 4.1 & 4.0 & 0.7 & 1.4 & 2.3 \\
\hline Ex-Jugoslawien & & 6.0 & 14.1 & 6.2 & 7.4 & 1.8 & 5.5 \\
\hline Deutschland & 35.1 & 33.2 & 8.8 & 6.7 & 9.9 & 14.2 & 13.4 \\
\hline Frankreich & 8.8 & 11.7 & 10.0 & 6.5 & 3.5 & 6.7 & 7.4 \\
\hline Österreich & & 1.0 & 2.3 & 1.8 & 1.7 & 5.7 & 3.6 \\
\hline $\begin{array}{l}\text { Sonstige EU-/ } \\
\text { EFTA-Staaten }\end{array}$ & 1.9 & 11.3 & 8.8 & 3.6 & 4.4 & 7.0 & 6.7 \\
\hline $\begin{array}{l}\text { Sonstige Industrie- } \\
\text { staaten }\end{array}$ & & 0.9 & 2.3 & & 2.1 & & 0.7 \\
\hline $\begin{array}{l}\text { Aussereurop. } \\
\text { Staaten }\end{array}$ & 15.0 & 17.0 & 16.1 & 23.4 & 13.6 & 3.7 & 11.6 \\
\hline \multicolumn{8}{|l|}{ Frauen } \\
\hline Italien & 1.9 & 11.4 & 9.0 & 20.9 & 28.4 & 42.9 & 26.8 \\
\hline Spanien & & 3.3 & 8.6 & 6.6 & 8.4 & 10.5 & 8.1 \\
\hline Portugal & 16.7 & 13.0 & 26.2 & 15.4 & 18.6 & 0.7 & 11.2 \\
\hline Türkei & 1.1 & 3.8 & 0.8 & 0.6 & 3.9 & 0.4 & 1.3 \\
\hline Ex-Jugoslawien & & 7.9 & 12.0 & 9.1 & 9.3 & 4.4 & 7.2 \\
\hline Deutschland & 56.2 & 22.6 & 11.7 & 5.3 & 9.9 & 16.5 & 15.4 \\
\hline Frankreich & 5.3 & 6.6 & 6.7 & 8.8 & 6.0 & 5.9 & 6.5 \\
\hline Österreich & 3.9 & 3.4 & 4.5 & 1.0 & 2.5 & 8.0 & 5.1 \\
\hline $\begin{array}{l}\text { Sonstige EU-/ } \\
\text { EFTA-Staaten }\end{array}$ & 14.4 & 7.4 & 6.0 & 8.1 & 2.6 & 5.2 & 6.0 \\
\hline $\begin{array}{l}\text { Sonstige Industrie- } \\
\text { staaten }\end{array}$ & & 2.0 & 1.1 & 0.4 & 0.3 & 0.7 & 0.9 \\
\hline $\begin{array}{l}\text { Aussereurop. } \\
\text { Stacten }\end{array}$ & 0.5 & 18.5 & 13.4 & 23.8 & 10.2 & 4.8 & 11.4 \\
\hline
\end{tabular}

Quelle: SAKE (1995), eigene Berechnungen

Anmerkungen: Zur Aufschlüsselung der Nationalitätengruppen siehe TAB. A8.2 
Tab. A8.7: Nationalitätengruppen nach Einwanderungskohorten (in Pro-

\begin{tabular}{|c|c|c|c|c|c|c|c|}
\hline \multirow[t]{2}{*}{$\begin{array}{l}\text { Nationalitäten- } \\
\text { gruppen }\end{array}$} & \multicolumn{6}{|c|}{$\begin{array}{c}\text { Einwanderungskohorten } \\
\text { (Aufenthaltsdauer in Jahren) }\end{array}$} & \multirow[t]{2}{*}{$\begin{array}{l}\text { Alter be } \\
\text { Einreise }\end{array}$} \\
\hline & $<1$ & $1-5$ & $5 \cdot 10$ & $10-15$ & $15-20$ & $>20$ & \\
\hline \multicolumn{8}{|l|}{ Männer } \\
\hline Italien & 1.2 & 2.4 & 7.9 & 11.1 & 7.0 & 70.5 & 20 \\
\hline Spanien & & 0.2 & 13.0 & 16.7 & 22.1 & 48.1 & 23 \\
\hline Portugal & 1.8 & 7.6 & 25.6 & 47.6 & 14.8 & 2.6 & 23 \\
\hline Türkei & & 7.8 & 30.3 & 31.7 & 2.8 & 27.5 & 23 \\
\hline Ex-Jugoslawien & & 9.1 & 43.1 & 20.7 & 12.4 & 14.7 & 23 \\
\hline Deutschland & 3.6 & 20.7 & 11.0 & 9.1 & 6.8 & 48.6 & 28 \\
\hline Frankreich & 1.7 & 13.3 & 22.8 & 16.0 & 4.4 & 41.9 & 26 \\
\hline Österreich & & 2.3 & 10.9 & 9.2 & 4.3 & 73.3 & 22 \\
\hline $\begin{array}{l}\text { Sonstige EU-/ } \\
\text { EFTA-Staaten }\end{array}$ & 0.4 & 14.0 & 21.9 & 9.7 & 5.9 & 48.0 & 26 \\
\hline $\begin{array}{l}\text { Sonstige Industrie- } \\
\text { staaten }\end{array}$ & & 11.0 & 58.2 & & 27.8 & 3.0 & 36 \\
\hline $\begin{array}{l}\text { Aussereurop. } \\
\text { Staaten }\end{array}$ & 1.8 & 12.3 & 23.4 & 36.9 & 10.8 & 14.8 & 26 \\
\hline \multicolumn{8}{|l|}{ Frauen } \\
\hline Italien & 0.2 & 6.7 & 5.7 & 10.7 & 9.5 & 67.2 & 23 \\
\hline Spanien & & 6.6 & 18.1 & 11.3 & 9.4 & 54.8 & 22 \\
\hline Portugal & 3.6 & 18.8 & 40.2 & 19.3 & 15.3 & 2.8 & 23 \\
\hline Türkei & 1.9 & 44.5 & 9.4 & 6.1 & 26.0 & 12.0 & 17 \\
\hline Ex-Jugoslawien & & 17.4 & 28.0 & 17.4 & 11.6 & 25.7 & 20 \\
\hline Deutschland & 8.6 & 23.1 & 12.8 & 4.7 & 5.8 & 45.0 & 26 \\
\hline Frankreich & 1.9 & 16.0 & 17.4 & 18.7 & 8.3 & 37.8 & 23 \\
\hline Österreich & 1.8 & 10.4 & 14.8 & 2.8 & 4.5 & 65.7 & 24 \\
\hline $\begin{array}{l}\text { Sonstige EU-/ } \\
\text { EFTA-Staaten }\end{array}$ & 5.6 & 19.5 & 16.7 & 18.3 & 3.9 & 36.0 & 26 \\
\hline $\begin{array}{l}\text { Sonstige Industrie- } \\
\text { staaten }\end{array}$ & & 36.3 & 21.0 & 6.0 & 2.7 & 34.0 & 33 \\
\hline $\begin{array}{l}\text { Aussereurop. } \\
\text { Staaten }\end{array}$ & 0.1 & 25.7 & 19.9 & 28.7 & 8.0 & 17.6 & 27 \\
\hline
\end{tabular}

Quelle: SAKE (1995), eigene Berechnungen

Anmerkungen: Zur Aufschlüsselung der Nationalitätengruppen siehe TAB. A8.2 
Tab. A9.1: Höchste abgeschlossene Ausbildung der erwerbstätigen Bevölkerung nach Nationalitätengruppen (in Prozent)

\begin{tabular}{|c|c|c|c|c|c|c|}
\hline \multirow{2}{*}{$\begin{array}{l}\text { Nationalitäten- } \\
\text { gruppen }\end{array}$} & \multicolumn{6}{|c|}{ Höchste Abgeschlossene Ausbildung } \\
\hline & 1 & 2 & 3 & 4 & 5 & 6 \\
\hline \multicolumn{7}{|l|}{ Männer } \\
\hline Italien & 38.3 & 29.4 & 10.1 & 5.1 & 3.3 & 13.9 \\
\hline Spanien & 50.3 & 28.2 & 7.5 & 4.2 & 0.2 & 9.6 \\
\hline Portugal & 52.6 & 20.1 & 8.9 & 1.0 & 1.9 & 15.5 \\
\hline Türkei & 37.8 & 33.8 & 8.0 & 0.5 & 6.6 & 13.3 \\
\hline Ex-Jugoslawien & 20.5 & 45.8 & 5.7 & 5.9 & 5.5 & 16.7 \\
\hline Deutschland & 1.2 & 29.8 & 11.1 & 24.4 & 30.4 & 2.9 \\
\hline Frankreich & 10.5 & 16.4 & 28.5 & 16.4 & 20.8 & 7.5 \\
\hline Österreich & 12.9 & 40.2 & 16.1 & 21.2 & 9.4 & 0.2 \\
\hline $\begin{array}{l}\text { Sonstige EU-/ } \\
\text { EFTA-Staaten }\end{array}$ & 1.7 & 20.7 & 7.1 & 9.2 & 50.2 & 11.1 \\
\hline $\begin{array}{l}\text { Sonstige } \\
\text { Industriestaaten }\end{array}$ & & 21.8 & & 3.3 & 69.5 & 5.4 \\
\hline $\begin{array}{l}\text { Aussereurop. } \\
\text { Staaten } \\
\end{array}$ & 7.6 & 20.3 & 13.6 & 11.3 & 29.5 & 17.7 \\
\hline \multicolumn{7}{|l|}{ Frauen } \\
\hline Italien & 48.4 & 19.9 & 8.4 & 0.7 & 2.5 & 20.1 \\
\hline Spanien & 59.4 & 11.3 & 12.6 & 3.8 & 1.0 & 11.9 \\
\hline Portugal & 59.9 & 9.4 & 7.7 & & 0.8 & 22.2 \\
\hline Türkei & 50.5 & 27.9 & 9.0 & & 12.5 & \\
\hline Ex-Jugoslawien & 33.9 & 38.5 & 10.1 & 2.7 & 4.8 & 10.1 \\
\hline Deutschland & 5.0 & 35.7 & 20.5 & 8.2 & 21.2 & 9.4 \\
\hline Frankreich & 10.0 & 13.3 & 36.7 & 12.8 & 17.5 & 9.6 \\
\hline Österreich & 14.8 & 37.2 & 7.2 & 6.6 & 12.0 & 22.1 \\
\hline $\begin{array}{l}\text { Sonstige EU-/ } \\
\text { EFTA-Staaten }\end{array}$ & 4.6 & 17.4 & 47.2 & 5.2 & 15.3 & 10.3 \\
\hline $\begin{array}{l}\text { Sonstige } \\
\text { Industriestaaten }\end{array}$ & 2.1 & & & & 87.4 & 10.5 \\
\hline $\begin{array}{l}\text { Aussereurop. } \\
\text { Staaten }\end{array}$ & 25.3 & 18.9 & 18.9 & 8.4 & 4.1 & 24.5 \\
\hline
\end{tabular}

Quelle: SAKE (1995), eigene Berechnungen

Anmerkungen: Erwerbstätige Bevölkerung: Personen zwischen 16 und 64 Jahren

Zur Aufschlüsselung der Nationalitätengruppen siehe TAB. A8.2

Höchste Abgeschlossene Ausbildung: 1 = Obligatorische Grundschule, 2 = Berufslehre, 3 = Vollzeitberufsschule, Diplommittelschule, Matura, $4=$ Höhere Berufsausbildung, 5 = Universität/Hochschule, 6 = Kein Abschluss, andere Ausbildung 
Tab. A9.3: Berufliche Stellung der erwerbstätigen Bevölkerung nach Nationalitätengruppen (in Prozent)

\begin{tabular}{|c|c|c|c|c|c|c|}
\hline \multirow{2}{*}{$\begin{array}{l}\text { Nationalitäten- } \\
\text { gruppen }\end{array}$} & \multicolumn{6}{|c|}{ Berufliche Stellung } \\
\hline & 1 & 2 & 3 & 4 & 5 & 6 \\
\hline \multicolumn{7}{|l|}{ Männer } \\
\hline Italien & 11.8 & 4.7 & 15.7 & 67.0 & 0.1 & 0.7 \\
\hline Spanien & 7.4 & 4.5 & 23.0 & 65.1 & & \\
\hline Portugal & 0.8 & 2.3 & 17.9 & 77.8 & 1.0 & \\
\hline Türkei & 7.3 & & & 92.7 & & \\
\hline Ex-Jugoslawien & 4.5 & 0.5 & 12.4 & 80.9 & 1.6 & \\
\hline Deutschland & 19.8 & 17.3 & 26.7 & 36.2 & & \\
\hline Frankreich & 14.1 & 16.4 & 29.4 & 38.9 & & 1.2 \\
\hline Österreich & 9.3 & 16.9 & 23.8 & 47.3 & & 2.7 \\
\hline $\begin{array}{l}\text { Sonstige EU-/ } \\
\text { EFTA-Staaten }\end{array}$ & 22.3 & 16.4 & 32.4 & 28.5 & & 0.4 \\
\hline $\begin{array}{l}\text { Sonstige } \\
\text { Industriestaaten }\end{array}$ & 12.2 & 19.6 & 17.9 & 50.2 & & \\
\hline $\begin{array}{l}\text { Aussereurop. } \\
\text { Staaten }\end{array}$ & 10.6 & 5.0 & 18.0 & 64.2 & & 2.2 \\
\hline \multicolumn{7}{|l|}{ Frauen } \\
\hline Italien & 6.5 & 3.1 & 5.0 & 85.4 & & \\
\hline Spanien & 2.1 & & 2.3 & 94.6 & 1.0 & \\
\hline Portugal & 2.4 & 0.6 & 2.1 & 94.0 & & 0.9 \\
\hline Türkei & & 21.1 & 6.5 & 72.3 & & \\
\hline Ex-Jugoslawien & 4.0 & 0.6 & 13.0 & 82.4 & & \\
\hline Deutschland & 12.1 & 4.2 & 20.3 & 59.4 & & 4.0 \\
\hline Frankreich & 8.4 & 1.6 & 9.3 & 80.7 & & \\
\hline Österreich & 5.2 & 3.1 & 14.8 & 76.8 & & \\
\hline $\begin{array}{l}\text { Sonstige EU-/ } \\
\text { EFTA-Staaten }\end{array}$ & 20.8 & 8.8 & 21.3 & 49.1 & & \\
\hline $\begin{array}{l}\text { Sonstige } \\
\text { Industriestaaten }\end{array}$ & 15.6 & 5.7 & 12.1 & 66.6 & & \\
\hline $\begin{array}{l}\text { Aussereurop. } \\
\text { Staaten }\end{array}$ & 5.1 & 0.6 & 3.4 & 87.0 & 3.9 & \\
\hline
\end{tabular}

Quelle: SAKE (1995), eigene Berechnungen

Anmerkungen: Erwerbstätige Bevölkerung: Personen zwischen 16 und 64 Jahren

Zur Aufschlüsselung der Nationalitätengruppen siehe TAB. A8.2

Berufliche Stellung: $I$ = Selbständige und mitarbeitende Familienangehörige, 2 = Arbeit nehmer in Unternehmensleitung, $3=$ Arbeitnehmer mit Vorgesetztenfunktion, $4=$ Arbeit nehmer ohne Vorgesetztenfunktion, $5=$ Lehrlinge, $6=$ Andere Erwerbstätige (keine $\mathrm{Zu}$ ordnung möglich) 
Tab. A9.5: Erwerbstätige Bevölkerung nach Wirtschaftszweigen und Nationalitätengruppen (in Prozent)

\begin{tabular}{|c|c|c|c|c|c|c|c|c|c|}
\hline \multirow{2}{*}{$\begin{array}{l}\text { Nationalitäten- } \\
\text { gruppen }\end{array}$} & \multicolumn{9}{|c|}{ Wirtschaftszweige } \\
\hline & 1 & 2 & 3 & 4 & 5 & 6 & 7 & 8 & 9 \\
\hline \multicolumn{10}{|l|}{ Männer } \\
\hline Italien & 0.9 & 0.5 & 38.8 & 24.2 & 18.0 & 3.1 & 7.8 & 5.4 & 1.3 \\
\hline Spanien & & & 35.5 & 29.8 & 12.1 & 2.6 & 8.3 & 11.3 & 0.4 \\
\hline Portugal & 2.1 & & 23.4 & 36.6 & 17.2 & 3.7 & 8.3 & 8.2 & 0.5 \\
\hline Türkei & & & 45.6 & & 15.9 & 25.4 & 0.5 & 12.5 & \\
\hline Ex-Jugoslawien & & & 40.3 & 23.9 & 17.5 & 6.5 & 3.1 & 8.7 & \\
\hline Deutschland & & & 36.1 & 5.3 & 16.7 & & 15.2 & 25.8 & 0.9 \\
\hline Frankreich & 2.1 & & 27.2 & 5.2 & 20.2 & 2.0 & 16.2 & 22.6 & 4.4 \\
\hline Österreich & & & 31.0 & 15.2 & 36.4 & & 11.9 & 3.7 & 1.8 \\
\hline $\begin{array}{l}\text { Sonstige EU-/ } \\
\text { EFTA-Staaten }\end{array}$ & & & 11.3 & 5.1 & 17.2 & 2.6 & 25.6 & 36.2 & 2.0 \\
\hline $\begin{array}{l}\text { Sonstige } \\
\text { Industriestaaten }\end{array}$ & & & & 21.8 & & & 17.6 & 46.4 & 14.2 \\
\hline $\begin{array}{l}\text { Aussereurop. } \\
\text { Staaten }\end{array}$ & 0.3 & & 31.6 & 8.5 & 23.3 & 4.2 & 14.0 & 14.0 & 4.1 \\
\hline \multicolumn{10}{|l|}{ Frauen } \\
\hline Italien & 0.4 & & 34.1 & & 27.3 & 3.2 & 15.8 & 18.5 & 0.8 \\
\hline Spanien & & & 23.8 & & 20.9 & 5.3 & 10.4 & 39.5 & 0.2 \\
\hline Portugal & & & 14.7 & & 38.1 & 2.9 & 8.4 & 34.4 & 1.5 \\
\hline Türkei & & & 42.9 & & 23.5 & & & 27.1 & 6.0 \\
\hline Ex-Jugoslawien & & & 35.4 & & 28.4 & 6.2 & 2.1 & 27.9 & \\
\hline Deutschland & & & 15.1 & & 13.0 & 6.7 & 11.0 & 52.3 & 2.0 \\
\hline Frankreich & & & 9.1 & 0.8 & 24.2 & 5.9 & 8.6 & 38.5 & 12.9 \\
\hline Österreich & & & 8.9 & & 27.5 & & 17.1 & 46.5 & \\
\hline $\begin{array}{l}\text { Sonstige EU-/ } \\
\text { EFTA-Staaten }\end{array}$ & & & 3.8 & & 20.3 & 2.6 & 3.6 & 54.1 & 15.5 \\
\hline $\begin{array}{l}\text { Sonstige } \\
\text { Industriestaaten }\end{array}$ & & & 4.7 & & 11.7 & & 5.7 & 61.2 & 16.8 \\
\hline $\begin{array}{l}\text { Aussereurop. } \\
\text { Staaten }\end{array}$ & & & 23.5 & & 19.8 & 1.8 & 18.8 & 33.3 & 2.8 \\
\hline
\end{tabular}

Quelle: SAKE (1995), eigene Berechnungen

Anmerkungen: Erwerbstätige Bevölkerung: Personen zwischen 16 und 64 Jahren

Zur Aufschlüsselung der Nationalitätengruppen siehe TAB. A8.2

Wirtschaftszweige: 1 = Land-, Forstwirtschaft, 2 = Energie-Wasserversorgung, Bergbau, 3 = Verarbeitende Produktion, $4=$ Baugewerbe, $5=$ HandeVGastgewerbe, $6=$ Verkehr/Nachrichten, 7 = Banken/Versicherungen/Immobilien, $8=$ Sonstige Dienstleistungen (Gesundheitswesen, Erziehung, Forschung), 9 = Öffentliche Verwaltung 
Tab. A9.7: Erwerbsbeteiligung und Beschäftigungsgrad nach Nationalitätengruppen (in Prozent)

\begin{tabular}{|l|c:cc|c|ccc|}
\hline $\begin{array}{l}\text { Nationalitäten- } \\
\text { gruppen }\end{array}$ & EQ & \multicolumn{3}{c|}{$\begin{array}{c}\text { Beschäftigungsgrad } \\
\text { (Männer) }\end{array}$} & $\boldsymbol{E Q}$ & \multicolumn{3}{c|}{$\begin{array}{c}\text { Beschäftigungsgrad } \\
\text { (Frauen) }\end{array}$} \\
\hline Italien & 92.8 & $20-69$ & $70-100$ & & 20 & $20-69$ & $70-100$ \\
Spanien & 95.3 & 1.6 & 98.4 & 67.3 & 5.6 & 39.5 & 54.9 \\
Portugal & 98.1 & 3.6 & 96.4 & 81.0 & 2.4 & 25.7 & 72.0 \\
Türkei & 95.2 & 0.9 & 99.1 & 89.0 & 4.2 & 18.7 & 77.1 \\
Ex-Jugoslawien & 94.6 & 2.9 & 97.1 & 52.6 & & 18.7 & 81.3 \\
Deutschland & 94.2 & 3.0 & 97.0 & 85.8 & 0.6 & 20.4 & 79.0 \\
Frankreich & 99.5 & 1.5 & 98.5 & 68.0 & 11.5 & 30.6 & 57.9 \\
Österreich & 96.6 & 2.0 & 98.0 & 79.6 & 2.7 & 29.1 & 68.2 \\
Sonstige EU-/ & 96.4 & 0.7 & 99.3 & 69.4 & 4.9 & 35.4 & 59.7 \\
EFTA-Staaten & & 3.1 & 96.9 & 76.3 & & 23.7 & 76.3 \\
Sonstige & 100.0 & & & & & & \\
Industriestaaten & & 3.3 & 96.7 & 80.4 & & 44.5 & 55.5 \\
$\begin{array}{l}\text { Aussereurop. } \\
\text { Staaten }\end{array}$ & 95.7 & & & & & & \\
\hline
\end{tabular}

Quelle: SAKE (1995), eigene Berechnungen

Anmerkungen: $E Q=$ Erwerbsquote: Erwerbspersonen (Erwerbstätige und Erwerbslose gemäss Definition SAKE) / Erwerbsfähige Bevölkerung (Personen im Alter zwischen 16 und 64 Jahren)

Zur Aufschlüsselung der Nationalitätengruppen siehe TAB. A8.2 
Tab. A9.9: Erwerbslosenquoten und Dauer der Erwerbslosigkeit nach Nationalitätengruppen (in Prozent)

\begin{tabular}{|c|c|c|c|c|c|}
\hline \multirow{2}{*}{$\begin{array}{l}\text { Nationalitäten- } \\
\text { gruppen }\end{array}$} & \multirow[t]{2}{*}{$E L Q$} & \multicolumn{4}{|c|}{ Erwerbslosigkeitsdauer (in Monaten) } \\
\hline & & $<3$ & $3-12$ & $12-24$ & $>24$ \\
\hline Männer & & & & & \\
\hline Italien & 4.8 & 21.1 & 18.4 & 60.4 & \\
\hline Spanien & 1.8 & & 33.7 & 66.3 & \\
\hline Portugal & 13.5 & 28.4 & 42.9 & 8.3 & 20.5 \\
\hline Türkei & 11.2 & & 76.3 & & 23.7 \\
\hline Ex-Jugoslawien & 11.4 & 23.1 & 31.8 & 26.9 & 18.2 \\
\hline Deutschland & 0.8 & 35.3 & 14.5 & 50.1 & \\
\hline Frankreich & 4.3 & 100.0 & & & \\
\hline Österreich & 5.2 & & & 100.0 & \\
\hline $\begin{array}{l}\text { Sonstige EU-/ } \\
\text { EFTA-Staaten }\end{array}$ & 1.6 & & 100.0 & & \\
\hline $\begin{array}{l}\text { Sonstige } \\
\text { Industriestaaten }\end{array}$ & 5.9 & & 100.0 & & \\
\hline $\begin{array}{l}\text { Aussereurop. } \\
\text { Staaten }\end{array}$ & 8.3 & 24.1 & 43.9 & 11.9 & 20.1 \\
\hline Frauen & & & & & \\
\hline Italien & 8.0 & 17.0 & 19.9 & 46.3 & 16.8 \\
\hline Spanien & 10.1 & 12.9 & 52.5 & 34.6 & \\
\hline Portugal & 9.6 & 47.4 & 38.1 & 14.5 & \\
\hline Türkei & 4.5 & & 100.0 & & \\
\hline Ex-Jugoslawien & 9.1 & & 36.7 & 12.5 & 50.8 \\
\hline Deutschland & 2.9 & & 71.6 & 4.9 & 23.5 \\
\hline Frankreich & 1.0 & & 100.0 & & \\
\hline Österreich & 0.0 & & & & \\
\hline $\begin{array}{l}\text { Sonstige EU-/ } \\
\text { EFTA-Staaten }\end{array}$ & 0.0 & & & & \\
\hline $\begin{array}{l}\text { Sonstige } \\
\text { Industriestaaten }\end{array}$ & 0.0 & & & & \\
\hline $\begin{array}{l}\text { Aussereurop. } \\
\text { Staaten }\end{array}$ & 14.5 & 47.1 & 36.6 & 1.3 & 15.0 \\
\hline
\end{tabular}

Quelle: SAKE (1995) eigene Berechnungen

Anmerkungen: $E L Q=$ Erwerbslosenquote: Enwerbslose (gemäss Definition SAKE) / Enwerbspersonen im Alter zwischen 16 und 64 (Enwerbstätige und Erwerbslose gemäss Definition SAKE)

Zur Aufschlüsselung der Nationalitätengruppen siehe TAB. A8.2 


\begin{tabular}{|c|c|c|c|}
\hline Code & Definition der Berufsgruppe & Code & Definition der Berufsgruppe \\
\hline 0 & Land- und Forstwirtschaft & $55 / 56$ & Einzel- und Detailhandel \\
\hline 01 & Landwirtschaft & 57 & Gastgewerbe \\
\hline 02 & Gartenbau & 58 & Reperaturgewerbe \\
\hline 03 & Forstwirtschaft & 6 & Verkehr, Nachrichtenïbermittlung \\
\hline 04 & Fischerei & 61 & Bahnen \\
\hline 1 & $\begin{array}{l}\text { Energie- und Wasserversorgung, } \\
\text { Bergbau }\end{array}$ & 62 & Strassenverkehr, Rohrleitung \\
\hline 11 & Energie- und Wasserversorgung & 63 & Schifffahrt \\
\hline 12 & Bergbau & 64 & Luftfahrt \\
\hline $2 / 3$ & Verarbeitende Produktion & 65 & $\begin{array}{l}\text { Verkehrsvermittlung, Spedition, La- } \\
\text { gerhäuser }\end{array}$ \\
\hline 21 & Herstellung von Nahrungsmitteln & 66 & Nachrichtenübermittlung \\
\hline 22 & Herstellung von Getränken & 7 & $\begin{array}{l}\text { Banken, Versicherungen, Immobi- } \\
\text { lien, Beratung }\end{array}$ \\
\hline 23 & Herstellung von Tabakwaren & 71 & Banken, Finanzgesellschaften \\
\hline 24 & Herstellung von Textilien & 72 & Versicherungen \\
\hline 25 & $\begin{array}{l}\text { Herstellung von Bekleidung und } \\
\text { Wäsche }\end{array}$ & 73 & Immobilien \\
\hline 26 & $\begin{array}{l}\text { Holzbe- und -verarbeitung, Herstel- } \\
\text { lung von Möbeln aus Holz }\end{array}$ & 74 & Vermietung, Leasing \\
\hline 27 & $\begin{array}{l}\text { Herstellung von Papier und Papier- } \\
\text { waren }\end{array}$ & 75 & $\begin{array}{l}\text { Beratung, Planung, kommerzielle } \\
\text { Dienste, Informatik }\end{array}$ \\
\hline 28 & $\begin{array}{l}\text { Herstellung graphischer Erzeugnisse, } \\
\text { Verlagswesen }\end{array}$ & 76 & Persönliche Dienstleistungen \\
\hline 29 & $\begin{array}{l}\text { Herstellung von Lederwaren und } \\
\text { Schuhen }\end{array}$ & 8 & Sonstige Dienstleistungen \\
\hline 31 & $\begin{array}{l}\text { Herstellung von chemischen Erzeug- } \\
\text { nissen }\end{array}$ & 81 & $\begin{array}{l}\text { Unterrichtswesen (privat und öffent- } \\
\text { lich) }\end{array}$ \\
\hline 32 & $\begin{array}{l}\text { Herstellung von Kunststoff- und } \\
\text { Kautschukwaren }\end{array}$ & 82 & $\begin{array}{l}\text { Forschung und Entwicklung, Prü- } \\
\text { fung (nicht an Hochschulen) }\end{array}$ \\
\hline 33 & $\begin{array}{l}\text { Abbau und Verarbeitung von Steinen } \\
\text { und Erden }\end{array}$ & 83 & $\begin{array}{l}\text { Gesundheits- und Veterinärwesen } \\
\text { (privat und öffentlich) }\end{array}$ \\
\hline 34 & Metallbearbeitung und -verarbeitung & 84 & Umweltschutz (privat und öffentlich) \\
\hline 35 & Maschinen- und Fahrzeugbau & 85 & $\begin{array}{l}\text { Heime, Wohlfahrtspflege (privat und } \\
\text { öffentlich) }\end{array}$ \\
\hline 36 & $\begin{array}{l}\text { Elektrotechnik, Elektronik, Feinme- } \\
\text { chanik, Optik }\end{array}$ & 86 & $\begin{array}{l}\text { Kirchliche, religiöse und weltan- } \\
\text { schauliche Vereinigungen }\end{array}$ \\
\hline
\end{tabular}


Tab. A9.17: Unterteilung der verschiedenen Branchen (Fortsetzung)

\begin{tabular}{|c|c|c|c|}
\hline 37 & $\begin{array}{l}\text { Herstellung von Uhren, Bijouterie- } \\
\text { wesen }\end{array}$ & 87 & $\begin{array}{l}\text { Dienstleistungen für die Allgemein- } \\
\text { heit, Interessenwahrung }\end{array}$ \\
\hline 38 & Sonstiges verarbeitendes Gewerbe & 88 & Kultur, Sport, Erholung \\
\hline 4 & Baugewerbe & 89 & Häusliche Dienste \\
\hline 41 & Bauhauptgewerbe (ohne Stahlbau) & 9 & Öffentliche Verwaltung \\
\hline 42 & $\begin{array}{l}\text { Ausbaugewerbe (ohne Holz- und } \\
\text { Metallverarbeitung) }\end{array}$ & 91 & Öffentliche Verwaltung i.e.S. \\
\hline 5 & $\begin{array}{l}\text { Handel, Gastgewerbe, Reperatur- } \\
\text { gewerbe }\end{array}$ & 92 & Sozialversicherung \\
\hline $\begin{array}{c}51 / 52 / \\
53\end{array}$ & Grosshandel & 93 & $\begin{array}{l}\text { Ausl. Vertretungen, intern. Organi- } \\
\text { sationen mit Behördencharakter }\end{array}$ \\
\hline 54 & Handelsvermittlung & & \\
\hline
\end{tabular}

Quelle: Bundesamt für Statistik, Systematik der Wirtschaftszweige

Tab. A9.18: Dissimilarity-Index zur Messung der beruflichen Segregation nach Nationalitätengruppen

\begin{tabular}{|c|c|c|c|c|c|c|}
\hline \multirow[t]{2}{*}{$\begin{array}{l}\text { Nationalitäten- } \\
\text { gruppen }\end{array}$} & \multicolumn{6}{|c|}{ DISSIMILARITY-INDEX D } \\
\hline & 1991 & 1995 & 1991 & 1995 & 1991 & 1995 \\
\hline Italien & 0.323 & 0.319 & 0.376 & 0.354 & 0.315 & 0.313 \\
\hline Spanien & 0.431 & 0.342 & 0.489 & 0.413 & 0.394 & 0.341 \\
\hline Portugal & 0.488 & 0.396 & 0.576 & 0.512 & 0.486 & 0.431 \\
\hline Türkei & 0.687 & 0.705 & 0.657 & 0.651 & 0.596 & 0.586 \\
\hline Ex-Jugoslawien & 0.553 & 0.479 & 0.679 & 0.597 & 0.493 & 0.532 \\
\hline Deutschland & 0.411 & 0.357 & 0.347 & 0.404 & 0.267 & 0.311 \\
\hline Frankreich & 0.369 & 0.377 & 0.425 & 0.382 & 0.340 & 0.315 \\
\hline Österreich & 0.441 & 0.500 & 0.646 & 0.519 & 0.433 & 0.411 \\
\hline $\begin{array}{l}\text { Sonstige EU-/ } \\
\text { EFTA-Staaten }\end{array}$ & 0.518 & 0.438 & 0.699 & 0.419 & 0.591 & 0.386 \\
\hline $\begin{array}{l}\text { Sonstige } \\
\text { Industriestaaten }\end{array}$ & 0.519 & 0.729 & 0.871 & 0.797 & 0.601 & 0.685 \\
\hline $\begin{array}{l}\text { Aussereurop. } \\
\text { Staaten }\end{array}$ & 0.466 & 0.418 & 0.397 & 0.380 & 0.337 & 0.330 \\
\hline
\end{tabular}

Quelle: SAKE (1995), eigene Berechnungen

Anmerkungen:Zur Aufschlüsselung der Nationalitätengruppen siehe TAB. A8.2 
Tab. A10.1: Einkommenshöhe der erwerbstätigen Bevölkerung nach Nationalitätengruppen (in Prozent)

\begin{tabular}{|c|c|c|c|c|c|c|c|}
\hline \multirow{2}{*}{$\begin{array}{l}\text { Nationalitäten- } \\
\text { gruppen }\end{array}$} & \multicolumn{7}{|c|}{ Einkommenshöhe } \\
\hline & 1 & 2 & 3 & 4 & 5 & 6 & 7 \\
\hline \multicolumn{8}{|l|}{ Männer } \\
\hline Italien & 0.5 & 3.2 & 14.6 & 43.7 & 27.1 & 8.7 & 2.1 \\
\hline Spanien & 1.0 & 3.8 & 15.6 & 42.6 & 26.4 & 9.7 & 1.0 \\
\hline Portugal & & 10.1 & 29.3 & 40.8 & 14.0 & 4.2 & 1.6 \\
\hline Türkei & 1.4 & 40.1 & 3.4 & 49.4 & 5.7 & & \\
\hline Ex-Jugoslawien & 1.9 & 8.4 & 31.8 & 31.7 & 19.6 & 6.6 & \\
\hline Deutschland & 1.4 & 1.4 & 3.7 & 14.8 & 27.9 & 35.5 & 15.3 \\
\hline Frankreich & 3.9 & & 10.6 & 23.8 & 13.0 & 35.0 & 13.8 \\
\hline Österreich & & & 6.6 & 31.1 & 6.4 & 31.3 & 24.7 \\
\hline $\begin{array}{l}\text { Sonstige EU-/ } \\
\text { EFTA-Staaten }\end{array}$ & & & 0.6 & 10.2 & 14.0 & 44.0 & 31.3 \\
\hline $\begin{array}{l}\text { Sonstige } \\
\text { Industriestaaten }\end{array}$ & & & & & 23.2 & & 76.8 \\
\hline $\begin{array}{l}\text { Aussereurop. } \\
\text { Staaten }\end{array}$ & 2.1 & 12.8 & 23.6 & 26.5 & 13.6 & 18.4 & 3.0 \\
\hline \multicolumn{8}{|l|}{ Frauen } \\
\hline Italien & 4.9 & 32.8 & 46.7 & 12.8 & 1.8 & 0.9 & \\
\hline Spanien & 0.4 & 23.2 & 46.9 & 26.2 & 2.9 & 0.3 & \\
\hline Portugal & 12.9 & 38.7 & 32.2 & 11.1 & 3.9 & 1.1 & \\
\hline Türkei & 19.4 & 67.8 & & & 12.8 & & \\
\hline Ex-Jugoslawien & 4.6 & 33.3 & 37.4 & 12.3 & 12.4 & & \\
\hline Deutschland & 1.9 & 5.8 & 12.1 & 30.4 & 42.0 & 7.2 & 0.7 \\
\hline Frankreich & & 13.6 & 29.6 & 13.0 & 32.2 & 11.6 & \\
\hline Österreich & 9.6 & 8.7 & 6.7 & 21.6 & 9.4 & 31.3 & 12.7 \\
\hline $\begin{array}{l}\text { Sonstige EU-/ } \\
\text { EFTA-Staaten }\end{array}$ & 2.2 & & 9.1 & 36.2 & 7.5 & 42.5 & 2.3 \\
\hline $\begin{array}{l}\text { Sonstige } \\
\text { Industriestaaten }\end{array}$ & & 26.7 & 13.6 & 4.9 & 14.2 & 40.7 & \\
\hline $\begin{array}{l}\text { Aussereurop. } \\
\text { Staaten }\end{array}$ & 13.9 & 13.8 & 32.7 & 14.8 & 16.8 & 7.9 & \\
\hline
\end{tabular}

Quelle: SAKE (1995), eigene Berechnungen

Anmerkungen: Erwerbstätige Bevölkerung: Personen zwischen 16 und 64 Jahren

Zur Aufschlüsselung der Nationalitätengruppen siehe TAB. A8.2

Einkommenshöhe in CHF: $1=<26^{\prime} 000 \mathrm{CHF}, 2=26^{\prime} 001-39^{\prime} 000 \mathrm{CHF}, 3=39^{\prime} 001-$ $52^{\prime} 000 \mathrm{CHF}, 4=52^{\prime} 001-65^{\prime} 000 \mathrm{CHF}, 5=65^{\prime} 001-78^{\prime} 000 \mathrm{CHF}, 6=78^{\prime} 001-130^{\prime} 000$ $\mathrm{CHF}, 7=>130^{\prime} 000 \mathrm{CHF}$ 


\section{LITERATURVERZEICHNIS}

Abrahamsen, Yngve/Kaplanek, Heinz/Schips, Bernd (1986): "Arbeitsmarkttheorie, Arbeitsmarktpolitik und Beschäftigung in der Schweiz", Beiträge zur empirischen Wirtschaftsforschung, Band 1, Verlag Rüegger: Grüsch.

Ahmad, Zeba/Flückiger, Yves (1995): "Analyse economique des differences de salaire entre femmes et hommes en Suisse", Vortrag an der SAKE-Benutzertagung, 18. Dezember 1994, Bern, mimeo.

Aigner, Dennis J./Cain, Glen G. (1977): "Statistical Theories of Discrimination in the Labor Market", Industrial and Labor Relations Review, 30 (2), 175-187.

Amemiya, Takeshi (1981): "Qualitative Response Models: A Survey", Journal of Economic Literature, 19 (4), 1483-1536.

Arrow, Kenneth J. (1973a): "The Theory of Discrimination", in: Ashenfelter, Orley A./Rees, A. (eds.): Discrimination in the Labor Markets, Princeton University Press: Princeton, 3-33.

Arrow, Kenneth J. (1973b): "Higher Education as a Filter", Journal of Public Economics, 2 (3), 193-216.

AS [Amtliche Sammlung des Bundesrechts] (Diverse Jahrgänge): Bern.

Ashenfelter, Orley A./Hannan, Timothy (1986): "Sex Discrimination and Product Market Competition: The Case of the Banking Industry", Quarterly Journal of Economics, 101 (February), 149-173.

Averitt, Robert T. (1968): The dual economy: the dynamics of American industry structure, W. W. Norton: New York.

Baltagi, Badi H. (1995): Econometric Analysis of Panel Data, John Wiley \& Sons: New York.

Barrett, Alan (1996): "Did the Decline Continue? Comparing the labor-market quality of United States immigrants from the late 1970's and 1980's", Journal of Population Economics, 9 (1), 55-63.

Bauer, Thomas (1995): "The Migration Decision with Uncertain Costs", Münchner Wirtschaftswissenschaftliche Beiträge, No. 95-25, Universität München.

Bauer, Thomas/Zimmermann, Klaus F. (1995a): "Network Migration of Ethnic Germans", Münchner Wirtschaftswissenschaftliche Beiträge, No. 95-16, Universität München.

Bauer, Thomas/Zimmermann, Klaus F. (1995b): "Modelling International Migration: Economic and Econometric Issues", in: Causes of International Mi- 
gration: Proceedings of a Workshop, Luxembourg, 14-16 December 1994, Eurostat: Brussels, 95-115.

Becker, Gary S. (1971): The Economics of Discrimination, 2nd ed., University of Chicago Press: Chicago \& London.

Becker, Gary S. (1962): "Investments in Human Capital: A Theoretical Analysis", Journal of Political Economy, 70, 9-49.

Beggs, John J./Chapman, Bruce J. (1991): "Male Immigrant Wage and Unemployment Experience in Australia", In: Abowd, John. M./Freeman, Richard B. (eds.): Immigration, Trade and the Labor Market, University of Chicago Press: Chicago, 369-384.

Ben Akiva, M./Lerman, S.R. (1985): Discrete Choice Analysis, Cambridge (Mass.): MIT Press.

Ben-Porath, Yoram (1973): "Labor Force Participation Rates and Labor Supply", Journal of Political Economy, 81, 697-704.

Bericht des Bundesrates (1991): Bericht des Bundesrates zur Ausländer- und Flüchtlingspolitik, 15. Mai 1991, Bern.

Berndt, Ernst R. (1996): The practice of econometrics: classic and contemporary, Reading (Mass.): Addison-Wesley.

BFA [Bundesamt für Ausländerfragen] (1998): Die Ausländer in der Schweiz Bestandsergebnisse, Retrospektive Bestandsergebnisse, Bevölkerungsbewegung: retrospektive Jahresergebnisse, Zentrales Ausländerregister: Bern.

BFF [Bundesamt für Flüchtlinge] (1998): Asylstatistik 1997, BFF: Bern.

BFS [Bundesamt für Statistik] (diverse Jahrgänge): Statistisches Jahrbuch der Schweiz, Verlag Neue Zürcher Zeitung: Zürich.

BFS [Bundesamt für Statistik] (1998a): Erwerbstätigenstatistik, Bern.

BFS [Bundesamt für Statistik] (1998b): Arbeitslosenstatistik, Bern.

BFS [Bundesamt für Statistik] (1996a): Statistisches Jahrbuch der Schweiz. Zürich: Verlag Neue Zürcher Zeitung.

BFS [Bundesamt für Statistik] (1996b): Die Schweizerische Arbeitskräfteerhebung - Konzepte, Methodische Grundlagen, Praktische Ausführung, Bern.

BFS [Bundesamt für Statistik] (1991a): Stichprobenplan und Gewichtung der SAKE 91, SAKE-News, 91/6, Bern.

BFS [Bundesamt für Statistik] (1991b): Terminologie im Bereich Erwerbsleben, SAKE-News, 91/4, Bern.

Bhagwati, Jagdish N./Srinivasan, T. (1974): "On Reanalyzing the Harris-Todaro Model: Rankings in the Case of Sector Specific Sticky Wages", American Economic Review, 64 (3), 502-508. 
Bickel, W. (1947): Bevölkerungsgeschichte der Schweiz seit dem Ausgang des Mittelalters, Zürich: Büchergilde Gutenberg.

BIGA (1964): Studienkommission für das Problem der ausländischen Arbeitskräfte: "Das Problem der ausländischen Arbeitskräfte", Bern.

BIGA/BFA (1991): Bericht über Konzeption und Prioritäten der schweizerischen Ausländerpolitik der Neunziger Jahre, Bern.

Blattner, Niklaus/Schwarz, Heinrich/Sheldon, George (1985): "Die Ausländerbeschäftigung als Determinante von Wirtschaftswachstum und Produktivität in einem Industrieland: Das Beispiel der Schweiz", in: Giersch, Herbert (ed.): Probleme und Perspektiven der weltwirtschaftlichen Entwicklung, Schriften des Vereins für Socialpolitik, Band 148, 367-381.

Blattner, Niklaus /Sheldon, George (1989): "Foreign Labour, Growth and Productivity: The Case of Switzerland", in: Gordon, Ian/Thirlwall, A.P. (eds.): European Factor Mobility - Trends and Consequences, MacMillan: London: 148-165.

Blattner, Niklaus /Theiss, Roland (1994): Ausländer und Arbeitslosigkeit, WWZStudie, Nr. 44, Universität Basel.

Blau, Francine D./Ferber, Marianne A. (1987): "Discrimination: Empirical Evidence from the United States", American Economic Review, 77 (2), 316320.

Blinder, Alan S. (1973): "Wage Discrimination: Reduced Form and Structural Estimates", Journal of Human Resources, 8 (4), 436-455.

Blinder, Alan S./Weiss, Yoram (1976): "Human Capital and Labor Supply: A Synthesis", Journal of Political Economy, 84 (3), 466-472.

Boisso, Dale/Hayes, Kathy/Hirschberger, Joseph/Silber, Jacques (1994): "Occupational segregation in the multidimensional case: decomposition and tests of significance", Journal of Econometrics, 61 (1), 161-171.

Borjas, George J. (1994a): "Immigration, Ethnic Identity, and Assimilation: The Intergenerational Transmission of Immigrant Skills", in: Giersch, Herbert (ed.): Economic Aspects of International Migration, Springer Verlag: Berlin, 139-154.

Borjas, George J. (1994b): "The Economics of Immigration", Journal of Economic Literature, 32 (4), 1667-1717.

Borjas, George J. (1993): "The Intergenerational Mobility of Immigrants", Journal of Labor Economics, 11 (1), 113-135.

Borjas, George J. (1992a): "Ethnic Capital and Intergenerational Mobility", Quarterly Journal of Economics, 17 (2), 123-150. 
Borjas, George J. (1992b): "National Origin and the Skills of Immigrants in the Postwar Period", in: Borjas, George J./Freeman, Richard B. (eds.): Immigration and the Work Force, The University of Chicago Press: Chicago, 1747.

Borjas, George J. (1991a): "Immigrants in the U.S. Labor Market: 1940-80", American Economic Review, 81 (2), 287-291.

Borjas, George J. (1991b): "Immigration and Self-Selection", in: Abowd, John. M./Freeman, Richard B. (eds.): Immigration, Trade and the Labor Market. University of Chicago Press: Chicago, 29-76.

Borjas, George J. (1990): Friends or Strangers: The Impact of Immigrants on the U.S. Economy, Basic Books: New York.

Borjas, George J. (1989a): "Immigrant and Emigrant Earnings: A longitudinal study", Economic Inquiry, 27 (1), 21-37.

Borjas, George J. (1989b): "Economic Theory and International Migration", International Migration Review, 23 (3), 457-485.

Borjas, George J. (1988): International differences in the labor market performance of immigrants, Kalamzoo: W.E. Upjohn Institute for Employment Research.

Borjas, George J. (1987): "Self-Selection and the Earnings of Immigrants", American Economic Review, 77 (4), 531-553.

Borjas, George J. (1985): "Assimilation, Changes in Cohort Quality, and the Earnings of Immigrants", Journal of Labor Economics, 3 (4), 463-489.

Borjas, George J. (1982): "The Earnings of Male Hispanic Immigrants in the United States", Industrial and Labor Relations Review, 35 (3), 343-353.

Borner, Silvio/Brunetti, Aymo/Straubhaar, Thomas (1994): Die Schweiz im Alleingang, Verlag Neue Zürcher Zeitung: Zürich.

Borner, Silvio/Brunetti, Aymo/Straubhaar, Thomas (1990): Die Schweiz AG: Vom Sonderfall zum Sanierungsfall? Verlag Neue Zürcher Zeitung: Zürich.

Borner, Silvio/Straubhaar, Thomas (1995): "Betreibt die Schweiz eine falsche Gastarbeiterpolitik? Freizügigkeit - ein Scheinproblem aus ökonomischer Sicht". Neue Zürcher Zeitung, Nr. 174, 29./30. Juli, 12.

Boyd, Monica (1989): "Family and Personal Networks in International Migration: Recent Developments and New Agendas", International Migration Review, 23 (3), 638-670.

Boymond, M. (1993): "L'inégalité de traitement envers les femmes sur le marché suisse du travail: mesures de la ségrégation professionelle et de la discrimination salariale", Thèse de doctorat, Université de Genève, non-publié. 
Brüderl, J./Diekmann, A/Engelhardt, H. (1993): "Einkommensunterschiede zwischen Frauen und Männern in der Schweiz", Schweizerische Zeitschrift für Soziologie, 19 (3), 573-588.

Buhmann, Brigitte (1992): "Die Schweizerische Arbeitskräfteerhebung: Einblick in die Arbeitswelt", Die Volkswirtschaft, 10, 42-48.

Buhmann, Brigitte/Hussmans, Ralf (1991): "Collecting traditional labour force data through modern techniques - The case of the Swiss Labour Force Survey", Bulletin of labour statistics, 1, ILO: Geneva, 9-19.

Burda, Michael C. (1995): "Migration and the Option Value of Waiting", CEPR Discussion Paper, 1229, London.

Bürgenmeier, Beat/Butare, Théo/Favarger, Philippe (1992): "Effects of Foreign Labour on the Prodution Pattern: The Swiss Case", Schweizerische Zeitschrift für Volkswirtschaft und Statistik, 128 (2), 103-124.

Butler, Richard J. (1987): "New Indices of Segregation", Economic Letters, 24, 359-362.

Cain, Glen C. (1986): "The Economic Analysis of Labor Market Discrimination: A Survey", in: Ashenfelter, Orley/Layard, Richard (eds.): Handbook of Labor Economics I, North-Holland: Amsterdam, 693-785.

Calvo, Guillermo A. (1978): "Urban Unemployment and Wage Determination in LDC's: Trade Unions in the Harris-Todaro Model", International Economic Review, 19, 65-81.

Carliner, Geoffrey (1980): "Wages, Earnings, and Hours of First, Second and Third Generation American Males", Economic Inquiry, 18 (January), 87102.

Carrington, William J./Detragiache, Enrica/Vishwanath, Tara (1996): "Migration with Endogenous Moving Costs", American Economic Review, 86 (4), 909930.

Charles, Maria (1987): "Geschlechtsspezifische Arbeitsmarktsegregation in der Schweiz", Schweizerische Zeitschrift für Soziologie, 13 (1), 1-27.

Chiodi-Ferro, Ilda (1994): Migration: Eine erweiterte ökonomische Analyse unter Berücksichtigung ausserökonomischer Erkenntnisse, Dissertation Universität Zürich, Verlag Hans Schellenberg: Winterthur.

Chiswick, Barry R. (1994): "The Performance of Immigrants in the United States Labor Market", in: Giersch, Herbert (ed.): Economic Aspects of International Migration, Springer Verlag: Berlin, 95-114.

Chiswick, Barry R. (1991): "Speaking, Reading, and Earnings among Low-skilled Immigrants", Journal of Labor Economics, 9 (2), 149-170. 
Chiswick, Barry R. (1988): "Differences in Education and Earnings across Racial and Ethnic Groups: Tastes, Discrimination, and Investments in Child Quality", Quarterly Journal of Economics, 103 (3), 571-597.

Chiswick, Barry R. (1986): "Human Capital and the Labor Market Adjustment of Immigrants: Testing of Alternative Hypotheses", in: Stark, Oded (ed.): Research in Human Capital and Development: Migration, 4, JAI Press: Greenwich, 1-26.

Chiswick, Barry R. (1980): "Immigrant Earnings Patterns by Sex, Race, and Ethnic Groupings", Monthly Labor Review, 103 (10), 22-25.

Chiswick, Barry R. (1979): "The Economic Progress of Immigrants: Some Apparently Universal Patterns", in: Fellner, William (ed.): Contemporary Economic Problems, Washington: American Enterprise Institute, 359-399.

Chiswick, Barry R. (1978): "The Effects of Americanization on the Earnings of Foreign-born Men", Journal of Political Economy, 86 (5), 897-921.

Chiswick, Barry R. (1977): "Sons of Immigrants: Are they at an Earnings Disadvantage?", American Economic Review, 67 (1), 376-380.

Chow, Gregory C. (1960): "Tests of Equality between Sets of Coefficients in Two Linear Regression Models", Econometrica, 28 (3), 591-605.

Collins, Susan M. (1991): "Immigrants, Labor Market Pressures, and the Composition of the Aggregate Demand", in: Abowd, John M./Freeman, Richard B. (eds.): Immigration, Trade and the Labor Market, University of Chicago Press: Chicago, 305-318.

Cordon, W.M./Findlay, Ronald (1975): "Urban Unemployment, Intersectoral Capital Mobility and Development Policy", Econometrica, 42 (165), 59-78.

Cornelius, Wayne A. (1976): "Mexican Migration to the United States: The View from Rural Sending Communities", Working Paper, Center for International Migration Studies, MIT.

Cornioley, Claude (1994): "Jugendarbeitslosigkeit in der Schweiz", Die Volkswirtschaft, 4, 51-56.

Cornioley, Claude (1992): "Zwei unterschiedliche Statistiken für die Messung der Arbeitslosigkeit in der Schweiz", Die Volkswirtschaft, 6, 28-33.

Cotton, Jeremiah (1988): "On the Decomposition of Wage Differentials", Review of Economics and Statistics, 70 (2), 236-243.

Cox, D./Oakes, R. (1984): Analysis of Survival Data, Chapman and Hall: London. Cross, H.E./Sandos, J.A. (1981): "Across the Border: Rural Development in Mexico and Recent Migration to the United States", Institute of Governmental Studies, University of California: Berkley. 
DaVanzo, Julie (1976): "Differences Between Return and non-Return Migration", International Migration Review, 38 (2), 13-27.

Deutsch, Joseph/Flückiger, Yves/Silber, Jacques (1994): "Measuring occupational segregation: Summary statistics and the impact of classification errors and aggregation", Journal of Econometrics, 61 (1), 133-146.

Dhima, Giorgio (1991): Politische Ökonomie der schweizerischen Ausländerregelung. Verlag Rüegger: Chur.

Diekmann, Andreas/Engelhardt, Henriette (1995): "Geschlechtsspezifische Einkommensungleichheiten und die Rolle des Familienstandes", Vortrag an der SAKE-Benutzertagung, 18.12.1994, Bern, mimeo.

Diekmann, Andreas/Engelhardt, Henriette (1994): "Einkommensungleichheit zwischen Frauen und Männern: Eine ökonometrische Analyse der Schweizer Arbeitskräfteerhebung", Schweizerische Zeitschrift für Volkswirtschaft und Statistik, 131 (1), 57-83.

Dixit, A.K./Pindyck, R.S. (1994): Investment under Uncertainty, Princeton University Press: Princeton, New Jersey.

Doeringer, Peter B./Piore, Michael J. (1971): International Labor Markets and Manpower Analysis, Lexington, Mass.: D.C. Heath.

Duleep, Harriet O./Regets, Mark C. (1996): "Admission Criteria and Immigrant Earnings Profiles", International Migration Review, 30 (2), 571-590.

Duncan, O.-D./Duncan, B. (1955): "A Methodological Analysis of Segregation Indices", American Sociological Review, 20 (2), 210-217.

Dustmann, Christian (1996): "Temporary Migration, Human Capital, and Language Fluency of Migrants", CEPR Discussion Paper, 1376, London.

Dustmann, C. (1993): "Earnings adjustment of temporary migrants", Journal of Population Economics, 6 (2), 153-168.

Eckey, Hans-Friedrich/Kosfeld, Reinhold/Dreger, Christian (1995): Ökonometrie - Grundlagen, Methoden, Beispiele, Gabler: Wiesbaden.

Ehrenberg, Ronald G./Smith, Robert S. (1994): Modern Labor Economics: Theory and Public Policy, Fifth Ed., Harper-Collins Publishers: New York.

Elster, Jon (1991): "Local Justice: How Institutions Allocate Scarce Goods and Necessary Burdens", European Economic Review, 35 (2-3), 273-291.

Expertenkommission Migration (1997): Ein neues Konzept der Migrationspolitik, Bern.

Fassmann, Heiner/Münz, Rainer (1996): Migration in Europa: historische Entwicklungen, aktuelle Trends und politische Reaktionen, Campus Verlag: Frankfurt, New York. 
Fawcett, James T. (1989): "Networks, Linkages, and Migration Systems", International Migration Review, 23 (3), 671-680.

Fawcett, James T./Arnold, F. (1987): "Explaining Diversity: Asian and Pacific Immigration Systems", in: Fawcett, James T./Carino, Benjamin V. (eds.): Pacific Bridges: The New Immigration from Asia and the Pacific Islands, Staten Island: Center for Migration Studies, 453-474.

Feninger, Rainer (1988): "Segmentation theories and their applicability for the labor market of the Federal Republic of Germany", in: Dams, Theodor/Mizuno, Masaichi (eds.): Employment Problems under the Conditions of Rapid Technological Change, Schriften zu Regional- und Verkehrsproblemen in Industrie- und Entwicklungsländern, Duncker-Humboldt: Berlin, 93-110.

Ferro-Luzzi, Giovanni (1994): "Inter-Industry Wage Differentials in Switzerland", Schweizerische Zeitschrift für Volkswirtschaft und Statistik, 130 (3), 421443.

Fields, G.S. (1975): "Rural-Urban Migration, Urban Unemployment and Underemployment, and Job Search Activity in LDC's", Journal of Development Economics, 2 (2), 165-188.

Fischer, Peter A./Straubhaar, Thomas (1996a): "Is Migration into EU-countries demand based?", in: Corry, Dan (ed.): Economics and European Union Migration Policy, Institute for Public Policy Research: London, 11-49.

Fischer, Peter A./Straubhaar, Thomas (1996b): "Einwanderung in die Schweiz ein polit-ökonomisches Lehrstück", in: Fassmann, Heiner/Münz, Rainer (eds.): Migration in Europa: historische Entwicklung, aktuelle Trends und politische Reaktionen, Campus Verlag: Frankfurt, New York, 183-207.

Flückiger, Yves/Boymond, Martine/Silber, Jacques (1995): "Ségrégation entre hommes et femmes sur le marché du travail: Une analyse sur la base de l'indice de Gini", Bundesamt für Statistik, Bern.

Franz, Wolfgang (1991): Arbeitsmarktökonomik, Springer-Verlag: Berlin.

Gabriel, Stuart A./Shack-Marquez, Janice/Wascher, William L. (1993): "Does migration arbitrage regional labor market differentials?", Regional Science and Urban Economics, 23 (2), 211-233.

Gang, Ira N./Zimmermann, Klaus F. (1996): "Is Child like Parent? Educational Attainment and Ethnic Origin", Münchner Wirtschaftswissenschaftliche Beiträge, 96-14, Universität München. 
Gerfin, Michael (1996): "Entwicklung von ökonometrischen Modellen zur Analyse der Dynamik auf dem schweizerischen Arbeitsmarkt", SAKE-News, 2/96, Bundesamt für Statistik: Bern.

Ghez, Gilbert R./Becker, Gary S. (1974): The Allocation of Time and Goods over the Life Cycle, New York: Columbia University Press for the National Bureau of Economic Research.

Gill, Andrew M. (1994): "Incorporating the Causes of Occupational Differences in Studies of Racial Wage Differentials", Journal of Human Resources, 29 (1), 20-41.

Golder, Stefan M. (1998): "On the employment performance of immigrant workers: an empirical analysis for Switzerland", Discussion Papers, 74, Institute for Economic Research Halle, Halle.

Golder, Stefan M. (1997a): "Social Security on the Verge of Collapse", WWZDiscussion Paper, 9708, Universität Basel.

Golder, Stefan M. (1997b): "Die Arbeitsmarktperformance von Einwanderern in der Schweiz: Eine theoretische und empirische Analyse im Rahmen eines internationalen Vergleichs", WWZ-Forschungsbericht, 6/97 (September), Universität Basel.

Golder, Stefan M./Straubhaar, Thomas (1998): "Migration to Switzerland: Some New Evidence", CEPR Discussion Paper, 1791, London.

Greene, William H. (1996): LIMDEP Version 7.0, User's Manual and Reference Guide.

Greene, William H. (1995): Econometric Analysis, Prentice-Hall: New Jersey.

Greenwood, Michael J. (1994): "Potential Channels of Immigrant Influence on the Economy of the Receiving Country", Papers in Regional Science, 73 (3), 211-240.

Greenwood, Michael J. (1985): "Human Migration: Theory, Models, and Empirical Studies", Journal of Regional Science, 25 (4), 521-544.

Greenwood, Michael J./Hunt, Gary L. (1994): "Economic Effects of Immigrants on Native and Foreign-Born Workers: Complementarity, Substitutability, and Other Channels of Influence", Southern Economic Journal, 61 (4), 1076-1097.

Greenwood, Michael J./McDowell, John M. (1994): "The National Labor Market Consequences of U.S. Immigration", in: Giersch, Herbert (ed.): Economic Aspects of International Migration, Springer Verlag: Berlin, 155-194. 
Greenwood, Michael J./McDowell, John M. (1986): "The Factor Market Consequences of U.S. Immigration", Journal of Economic Literature, 24 (4), 1738-1772.

Gregory, R.G./Anstie, R./Klug, E. (1991): "Why are low-skilled immigrants in the United States poorly paid relative to their australian counterparts?", in: Abowd, John M./Freeman, Richard B. (eds.): Immigration, Trade and the Labor Market. University of Chicago Press: Chicago, 385-406.

Grossen, Dieter (1996): "Ausländer und Wirtschaft", Vortrag anlässlich des Medienseminars des EJPD zur Ausländerpolitik, 16. Mai 1996, mimeo.

Gujarati, Damodar N. (1995): Basic Econometrics, McGraw-Hill: New York.

Hammar, Tomas (1985): European Immigration Policy: A Comparative Study, Cambridge: Cambridge University Press.

Harris, I./Todaro, M.P. (1970): "Migration, Unemployment and Development: A Two-Sector Analysis", American Economic Review, 60 (1), 126-142.

Haug, Werner (1995): Vom Einwanderungsland zur multikulturellen Gesellschaft - Grundlagen einer schweizerischen Migrationspolitik, Bundesamt für Statistik: Bern.

Heckman, James J. (1979): "Sample Selection Bias as a Specification Error", Econometrica, 47 (1), 153-162.

Heckman, James J. (1976a): "The Common Structure of Statistical Models of Truncation, Sample Selection Bias and Limited Dependent Variables and a Simple Estimator for Such Models", Annals of Economic and Social Measurement, 5 (4), 475-492.

Heckman, James J. (1976b): "A Life-Cycle Model of Earnings, Learnings and Consumption", Journal of Political Economy, 84 (4), 11-44.

Heckman, James J. (1974): "Shadow Prices, Market Wage and Labor Supply", Econometrica, 42 (4), 679-694.

Hendricks, Lutz (1997): "Migration and Human Capital", Arizona State University, Department of Economics, mimeo.

Hicks, John (1932): The Theory of Wages, London: MacMillan.

Hoffmann-Nowotny, Hans-Joachim (1985): "Switzerland", in: Hammar, Tomas (ed.): European Immigration Policy: A Comparative Study, Cambridge: Cambridge University Press, 206-236.

Hoffmann-Nowotny, Hans-Joachim/Kilias, Martin (1979): "Switzerland" in: Krane, R.E. (ed.): International Labor Migration in Europe, New York: Praeger, 45-62. 
Hsiao, Cheng (1993): Analysis of Panel Data, Cambridge University Press: Cambridge.

Hugo, G.J. (1981): "Village-Community Ties, Village Norms, and Ethnic and Social Networks: A Review of Evidence from the Third World", in: Dejong, G.F./Gardner, R.W. (eds.): Migration Decision Making: Multidisciplinary Approaches to Microlevel Studies in Developed and Developing Countries, New York: Pergamon Press, 186-225.

Hunt, Gary L. (1993): "Equilibrium and Disequilibrium in Migration Modelling", Regional Studies, 27 (4), 341-349.

Jasso, Guillermina/Rosenzweig, Mark R. (1995): "Do immigrants screened for skills do better than family reunification immigrants?", International Migration Review, 29 (1), 85-111.

Jenkins, Stephen P. (1994): "Earnings discrimination measurement: A distributional approach", Journal of Econometrics, 61 (1), 81-102.

Jones, F.L./Kelley, J. (1984): "Decomposing Differences between Groups - A Cautionary Note on Measuring Discrimination", Sociological Methods and Research, 12, 323-343.

Kalbfleisch, J./Prentice, R. (1980): The Statistical Analysis of Failure Time Data, John Wiley \& Sons: New York.

Kälin, Walter/Rieder, Andreas (1997): Einführung in das Ausländerrecht, mimeo.

Kalleberg, Arne L. (1986): "The Comparability Analysis of Labor Market Segmentation and Earnings Inequality", Arbeitspapier, 202, Sonderforschungsbereich 3, Mikroanalytische Grundlagen der Gesellschaftspolitik, J.W. Goethe Universität Frankfurt und Universität Mannheim.

Katz, E./Stark, Oded (1987): "International Migration under Asymmetric Information", Economic Journal, 97 (387), 718-726.

Kaufman, Bruce E. (1989): The economics of labor markets and labor relations, Dryden Press: Chicago.

Kennedy, Peter (1996): A Guide to Econometrics, Blackwell: Oxford.

Kerr, C. (1977): Labor Markets and Wage Determination, Berkley: University of California Press.

Kiefer, Nicholas M. (1988): "Economic Duration Data and Hazard Functions", Journal of Economic Literature, 26 (2), 646-679.

Killingsworth, Mark (1982): "'Learning by Doing' and 'Investment in Training': A Synthesis of Two Rival Models of the Life Cycle", Review of Economic Studies, 49 (2), 263-271. 
Klodt, Henning/Maurer, Rainer/Schimmelpfennig, Axel (1997): Tertiarisierung in der deutschen Wirtschaft, Kieler Studie, 283, Institut für Weltwirtschaft: Kiel.

Kohli, Ulrich (1997): "Trade and Migration: A Production-Theory Approach", CEPR Discussion Paper, 1684, London.

Kohli, Ulrich (1993): "International Labor Mobility and the Demand for Imports", Schweizerische Zeitschrift für Volkswirtschaft und Statistik, 129 (3), 547561.

Kossoudji, Sherrie A. (1989): "Immigrant Worker Assimilation: Is it a Labor Market Phenomenon?", Journal of Human Resources, 24 (3), 494-527.

Kugler, Peter (1988): "Lohndiskriminierung in der Schweiz: Evidenz von Mikrodaten", Schweizerische Zeitschrift für Volkswirtschaft und Statistik, 124 (1), 23-47.

Kwok, P./Leland, H. (1984): "Migration and Asymmetric Information: Reply", American Economic Review, 74 (3), 535.

Lang, Kevin/Dickens, William T. (1992): "Labor Market Segmentation, Wage Dispersion and Unemployment", NBER Working Paper, 4073.

Lehmann, S. (1949): Grundzüge der schweizerischen Ausländerpolitik, Bern.

Licht, Georg/Steiner, Viktor (1993): "Assimilation, Labour Market Experience, and Earnings Profiles of Temporary and Permanent Immigrant Workers in Germany", ZEW-Discussion Paper, 93-06, Mannheim.

Lundborg, Per (1991): "Determinants of Migration in the Nordic Labor Market". Scandinavian Journal of Economics, 93 (3), 363-375.

Maechler, Andréa M. (1993): "Assimilation and Earnings Dynamics in Switzerland: A Comparison Between Foreign Permanent Residents' And Native Workers' Earnings Profiles", Institute Universitaires des Hautes Etudes Internationales, Université de Genève, mimeo.

Malmberg, Gunnar/Fischer, Peter A. (1997): Immobility and Insider-Advantages: Empirical Evidence for Sweden, University of the Bundeswehr Hamburg, mimeo.

Marshall, F. Ray (1974): "The economics of racial discrimination: a survey", Journal of Economic Literature, 12, 849-871.

Massey, Douglas S. (1990a): "The Social and Economic Origins of Immigration", Annals of the American Academy of Political and Social Sciences, 510, 6072.

Massey, Douglas S. (1990b): "Social Structure, Household Strategies, and the Cummulative Causation of Migration", Population Index, 56, 1-26. 
Massey, Douglas et al. (1993): "Theories of International Migration: A Review and Appraisal", Population and Development Review, 19 (3), 431-466.

Massey, Douglas et al. (1987): Return to Aztlan: The Social Process of International Migration from Western Mexico, University of California Press: Berkley.

Massey, Douglas S./España, F.G. (1987): "The Social Process of International Migration", Science, 237, 733-738.

Mincer, Jacob (1978): "Family Migration Decisions", Journal of Political Economy, 86 (5), 749-773.

Mincer, Jacob (1974): Schooling, experience and earnings, NBER, New York.

Molho, Ian (1986): "Theories of Migration: A Review", Scottish Journal of Political Economy, 33 (4), 396-419.

Müller, Rudolf A. (1991): "Schweizerisches Beschäftigungswunder?", Die Volkswirtschaft, 1, 15-18.

Neumark, David (1988): "Employers' Discriminatory Behavior and the Estimation of Wage Discrimination", Journal of Human Resources, 23 (3), 279-295.

Nielsen, Helena S. (1998): "Two Notes on Discrimination and Decomposition", Working Paper, 98-01, Centre for Labour Market and Social Research, Aarhus.

North, David S./Houston, Marion F. (1976): The Characteristics and Role of Illegal Aliens in the U.S. Labor Market: An Explanatory Study, Linton: Washington D.C.

Oaxaca, Ronald L. (1973): "Male-Female Wage Differentials in Urban Labor Markets", International Economic Review, 14 (3), 693-709.

Oettinger, Gerald S. (1996): "Statistical Discrimination and the Early Career Evolution of the Black-White Wage Gap", Journal of Labor Economics, 14 (1), 52-78.

Olson, Mancur (1965): The Logic of Collective Action, Harvard University Press: Cambridge.

Piore, Michael J. (1979): Birds of Passage: Migrant Labor and Industrial Societies, Cambridge University Press: Cambridge.

Piore, Michael J. (1978): "Lernprozesse, Mobilitätsketten und Arbeitsmarktsegmente", in: Sengenberger, Werner (ed.): Der gespaltene Arbeitsmarkt, Frankfurt a.M./New York, 67-98.

Pischke, Jörn-Steffen (1992): "Assimilation and the Earnings of Guestworkers in Germany", ZEW-Discussion Paper, 92-17, Mannheim. 
Pischke, Jörn-Steffen/Velling, Johannes (1994): "Wage and Employment Effects of Immigration to Germany - An Analysis Based on Local Labour Markets". CEPR Discussion Paper, 935, London.

Portes, Alejandro/Bach, R.L. (1985): Latin Journey: Cuban and Mexican Immigrants in the United States, University of California Press: Berkley.

Portes, Alejandro/Böröcz, József (1989): "Contemporary Immigration: Theoretical Perspectives on its Determinants and Modes of Incorporation", International Migration Review, 23 (3), 606-630.

Puhani, Patrick A. (1997): "Foul or Fair? The Heckman Correction for Sample Selection and Its Critique: A Short Survey", ZEW-Discussion Paper, 97-07, Mannheim.

Raess-Eichenberger, Susanne/Raess-Eichenberger, Markus (1997): Das aktuelle schweizerische Ausländerrecht. Nachschlagewerk zu allen ausländerrechtlichen Belangen unter Berücksichtigung der aktuellen Rechtsprechung, Zürich: WEKA Verlag.

Ravenstein, E. (1889): "The Laws of Migration", Journal of the Statistical Society, 20 (3), 214-301.

Reimers, Cordelia (1983): "Labor Market Discrimination Against Hispanic and Black Men", Review of Economics and Statistics, 65 (4), 570-579.

Robinson, Joan (1933): Economics of Imperfect Competition, London: MacMillan.

Ronning, Gerd (1991): Mikroökonometrie, Springer-Verlag: Berlin.

Rosen, Sherwin (1986): "The Theory of Equalizing Differences", in: Ashenfelter, O./Layard, R. (eds.): Handbook of Labor Economics, Vol. 1, North-Holland: Amsterdam, 641-692.

Rosen, Sherwin (1977): "Human Capital: A Survey of Empirical Research", in: Ehrenberg, Ronald G. (ed.): Research in Labor Economics, Vol. 1, Greenwich: JAI Press.

Rossi, Angelo A./Leighton, Thomas R. (1971): "Inflation in the Post-war Swiss Economy - an Econometric Study of the Interaction between Immigration and the Labour Market", Schweizerische Zeitschrift für Volkswirtschaft und Statistik, 4, 761-790.

Roy, A.D. (1951): "Some thoughts on the Distribution of Earnings", Oxford Economic Papers, 3, 135-146.

Roy, A.D. (1950): "The Distribution of Earnings and of Individual Output", Economic Journal, 60 (3), 489-505. 
Schaeffer, Peter V. (1995): "The Work Effort and the Consumption of Immigrants as a Function of their Assimilation", International Economic Review, 36 (3), 625-642.

Schaeffer, Peter V. (1985): "Human Capital Accumulation and Job Mobility", Journal of Regional Science, 25 (1), 103-114.

Schlaepfer, R. (1969): Die Ausländerfrage in der Schweiz vor dem Ersten Weltkrieg, Dissertation, Universität Zürich, Juris Druck und Verlag.

Schmidt, Christoph M. (1997): "Immigrant Performance in Germany: Labor Earnings of Ethnic German Migrants and Foreign Earnings", The Quarterly Review of Ecnomics and Finance, 37 (Special Issue), 379-397.

Schmidt, Christoph M. (1994): "The Economic Performance of Germany's East European Immigrants", CEPR Discussion Paper, 963, London.

Schmidt, Christoph M. (1992): "Country-of-Origin Differences in the Earnings of German Immigrants", Münchner Wirtschaftswissenschaftliche Beiträge, 9229, Universität München.

Schmidt, Christoph M./Stilz, Anette /Zimmermann, Klaus F. (1994): "Mass Migration, Unions, and Government Intervention", Journal of Public Economics, 55 (2), 185-201.

Schultz, T.W. (1975): "The Value of the Ability to Deal with Disequilibria", Journal of Economic Literature, 13 (3), 827-846.

Schwartz, Aba (1976): "Migration, Age, and Education", Journal of Political Economy, 84 (4), 701-719.

Schwarz, Heinrich (1988): Volkswirtschaftliche Wirkungen der Ausländerbeschäftigung in der Schweiz, Basler Sozialökonomische Studien, Band 31, Verlag Rüegger: Grüsch.

Schwarz, Heinrich (1985): "Regionale Wirkungen der Fremdarbeiterpolitik", in: Brugger, Ernst/Frey, René L. (eds.): Sektoralpolitik versus Regionalpolitik, Verlag Rüegger: Grüsch, 405-419.

Seifert, Wolfgang (1993): "Arbeitsmarktsegmentation - Mobilitätsbarriere für ausländische Arbeitnehmer?", Arbeitsgruppe Sozialberichtserstattung, 93102, Wissenschaftszentrum Berlin für Sozialforschung.

Sengenberger, Werner (1978): "Einführung: Die Segmentation des Arbeitsmarktes als politisches und wissenschaftliches Problem", in: Sengenberger, Werner (ed.): Der gespaltene Arbeitsmarkt: Probleme der Arbeitsmarktsegmentation, Frankfurt a.M., 15-42. 
Sheldon, George (1993): "Konjunkturelle und strukturelle Aspekte des schweizerischen Arbeitsmarktes", Studie Nr. 16, Bundesamt für Konjunkturfragen: Bern.

Shields, G.M./Shields, M.P. (1989): "The Emergence of Migration Theory and a Suggested New Direction", Journal of Economic Surveys, 3 (4), 277-304.

Silber, Jacques (1989a): "Factor components, population subgroups and the computation of the Gini index of inequality", Review of Economics and Statistics, 71(1), 107-115.

Silber, Jacques (1989b): "On the Measurement of Employment Segregation", Economic Letters, 30 (3), 237-243.

Sjaastad, Larry A. (1962): "The Costs and Returns of Human Migration", Journal of Political Economy, 70, 80-93.

Smith, Adam (1776): An Inquiry into the Nature and Causes of the Wealth of Nations, London: Strahan/Cadell.

SOPEMI (1995): Trends in International Migration, Annual Report 1994, OECD: Paris.

Spence, Michael A. (1973): "Job Market Signalling", Quarterly Journal of Economics, 87 (3), 355-374.

Spence, Michael A. (1974): Market Signalling Informational Transfer in Hiring and Related Screening Processes, Cambridge, Mass.: Harvard University Press.

Stark, Oded (1991): The migration of labor, Basil Blackwell: Cambridge, Mass.

Stark, Oded (1984a): "Rural-to-Urban Migration in LDCs: A Relative Deprivation Approach", Economic Development and Cultural Change, 32, 475-486.

Stark, Oded (1984b): "Discontinuity and the Theory of International Migration", Kyklos, 37 (2), 206-222.

Stark, Oded (1983): "Migration Decision Making: A Review Essay", Migration and Development Program Discussion Paper Series, 2, Cambridge (Mass.): Harvard University.

Stark, Oded/Bloom, David E. (1985): "The New Economics of Labor Migration", American Economic Review, Papers and Proceedings, 75 (2), 173-178.

Stark, Oded/Levhari, D. (1982): "On Migration and Risk in LDCs", Economic Development and Cultural Change, 31 (1), 191-196.

Stark, Oded/Taylor, J.E. (1988): "Relative Deprivation and International Migration", Migration and Development Program Discussion Paper Series, 36, Cambridge (Mass.): Harvard University. 
Stark, Oded/Yitzhaki, S. (1988): "Migration and Relative Deprivation", in: Maital (ed.): Applied Behavirol Economics, Brighton: Wheatsheaf Books.

Steineck, Alexander (1994): "The transferability of human capital: some empirical evidence for West Germany", International Review of Applied Economics, 8 (2), 197-226.

Steiner, Viktor/Velling, Johannes (1992): "Re-Migration Behaviour and Expected Duration of Stay of Guest-Workers in Germany", ZEW-Discussion Paper, 92-14, Mannheim.

Stewart, Mark B. (1983): "Racial Discrimination and Occupational Attainment in Britain", Economic Journal, 93, 521-541.

Stewart, James B./Hyclak, Thomas (1984): "An Analysis of Earnings Profiles of Immigrants", Review of Economics and Statistics, 66 (2), 292-296.

Stiglitz, Joseph E. (1974): "Alternative Theories of Wage Determination and Unemployment in LDC's: The Labor-Turnover Model", Quarterly Journal of Economics, 88 (2), 194-227.

Straubhaar, Thomas (1992): "Internationale Faktorwanderungen: Substitutionalität versus Komplementarität", Wirtschaftswissenschaftliches Studium, 11, 563570.

Straubhaar, Thomas (1991): Schweizerische Ausländerpolitik im Strukturwandel - Strukturberichterstattung zuhanden des Bundesamtes für Konjunkturfragen (BFK), Bern: BFK.

Straubhaar, Thomas (1989): "Grundzüge einer schweizerischen Migrationspolitik", Wirtschaftspolitische Mitteilungen, 45 (9/10).

Straubhaar, Thomas (1988): On the Economics of International Labor Migration, Verlag Paul Haupt: Bern.

Straubhaar, Thomas (1986): "The Causes of International Labor Migrations - A Demand-Determined Approach", International Migration Review, 20 (4), 835-855.

Straubhaar, Thomas/Weber, René (1994): "On the Economics of Immigration: Some Empirical Evidence for Switzerland", International Review of Applied Economics, 8 (2), 107-129.

Taubmann, Paul/Wachter, Michael L. (1986): "Segmented Labor Markets", in: Ashenfelter, Orley/Layard, Richard (eds.): Handbook of Labor Economics, Vol. II, North-Holland: Amsterdam, 1183-1217.

Terrell, Katherine (1992): "Female-male earnings differentials and occupational structure", International Labour Review, 131 (4-5), 387-404.

Thurow, Lester (1975): Generating Inequality, Basic Books: New York. 
Tidwick, Kathryn (1976): "Need for Achievement, Social Class and Intention to Emigrate in Jamacian Studies", Social and Economic Studies, March, 52-60. Todaro, M.P. (1980): "Internal Migration in Developing Countries: A Survey", in: Easterlin, Richard A. (ed.): Population and Economic Change in Developing Countries, Chicago: NBER, 361-402.

Todaro, M.P. (1969): "A Model of Labor Migration and Urban Unemployment in Less Developed Countries", American Economic Review, 59 (1), 138-148.

Todaro, M.P. (1968): "An Analysis of Industrialization, Employment and Unemployment in LDC's", Yale Economic Essays, 8 (2), 329-402.

Veall, M.R./Zimmermann, K.F. (1992): "Pseudo- $\mathrm{R}^{2} \mathrm{~s}$ in the Ordinal Probit Model", Journal of Mathematical Sociology, 16, 333-342.

Velling, Johannes (1995a): Immigration und Arbeitsmarkt: Eine empirische Analyse für die Bundesrepublik Deutschland, Baden-Baden: Nomos Verl.-Ges.

Velling, Johannes (1995b): "Wage Discrimination and Occupational Segregation of Foreign Male Workers in Germany", ZEW-Discussion Paper, 95-04, Mannheim.

Velling, Johannes/Woydt, Malte (1993): "Die Migrationspolitiken in ausgewählten Industrieländern - ein synoptischer Vergleich", ZEW-Dokumentation, Mannheim.

Vijverberg, Wim P.M. (1993): "Labor Market Performance as a Determinant of Migration", Economica, 60 (238), 143-160.

Weber, René (1993): Einwanderung und staatliche Umverteilung: Eine ökonomische Wirkungsanalyse für die Schweiz, WWZ-Beiträge, Band 14, Verlag Rüegger: Chur.

Weber, René/Straubhaar, Thomas (1994): "Budget Incidence of Immigration into Switzerland: A Cross-Section Analysis of the Public Transfer System", CEPR Discussion Paper, 934, London.

Wehrli, Christoph (1996): "Ausländerpolitik auf wackeligen Füssen: Rechtsgutachten gegen das Drei-Kreise-Modell", Neue Zürcher Zeitung, Internationale Ausgabe, 7. August 1996, Nr. 181, 23.

Weiss, Yoram (1986): "The Determinants of Life Cycle Earnings: A Survey", in: Ashenfelter, Orley/Levich, Richard (eds.): The Handbook of Labor Economics, Vol. 1, North Holland: Amsterdam, 603-639.

White, H. (1980): "A Heteroscedasticity Consistent Variance Covariance Matrix Estimator and Direct Test of Heteroscedasticity", Econometrica, 48 (4), 817 818. 
Windmeijer, F.A. (1995): "Goodness of Fit Measures in Binary Choice Models", Econometric Reviews, 14(1), 101-116.

Wolter, Achim (1996): "Multinationale Unternehmen als Kanal der Wanderung Höherqualifizierter", Diskussionsbeiträge zur Wirtschaftspolitik, Nr. 64, Institut für Wirtschaftspolitik, Universität der Bundeswehr Hamburg.

Yinger, John (1986): "Measuring Discrimination with Fair Housing Audits: Caught in the Act", American Economic Review, 76 (5), 881-893.

Zimmermann, Klaus F. (1997): "Die Arbeitsmarktkonsequenzen unterschiedlicher Einwanderungspolitiken", in: Sadowski, Dieter/Pull, Kerstin (eds.): Vorschläge jenseits der Lohnpolitik, Frankfurt: Campus-Verlag, 297-316.

Zimmermann, Klaus F. (1994): "Immigration Politics in Europe: An Overview", in: Siebert, Horst (ed.): Migration: A Challenge for Europe. Mohr: Tübingen, 227-258.

Zimmermann, Klaus F. (1993): "Ökonomische Konsequenzen der Migration für den heimischen Arbeitsmarkt", Schweizerische Zeitschrift für Volkswirtschaft und Statistik, 129 (3), 283-301. 
Stefan Golder - 978-3-631-74998-2

Downloaded from PubFactory at 01/11/2019 09:25:33AM

via free access 


\section{SCHRIFTEN ZUR WIRTSCHAFTSTHEORIE UND WIRTSCHAFTSPOLITIK}

Herausgegeben von Rolf Hasse, Wolf Schäfer, Thomas Straubhaar, Klaus W. Zimmermann

Band 1 Lars Bünning: Die Konvergenzkriterien des Maastricht-Vertrages unter besonderer Berücksichtigung ihrer Konsistenz. 1997.

Band 2 Andreas Henning: Beveridge-Kurve, Lohnsetzung und Langzeitarbeitslosigkeit. Eine theoretische Untersuchung unter Berücksichtigung des Insider-Outsider-Ansatzes und der Entwertung des Humankapitals. 1997.

Band 3 Iris Henning: Die Reputation einer Zentralbank. Eine theoretische Untersuchung unter besonderer Berücksichtigung der Europäischen Zentralbank. 1997.

Band 4 Rüdiger Hermann: Ein gemeinsamer Markt für Elektrizität in Europa. Optionen einer Wettbewerbsordnung zwischen Anspruch und Wirklichkeit. 1997.

Band 5 Alexander Tiedtke: Japan und der Vorwurf des Trittbrettfahrerverhaltens in der US-amerikanisch-japanischen Allianz. 1997.

Band 6 Wolfgang Grimme: Ordnungspolitisches Konzept der Regionalpolitik. Darstellung der Defizite und des Reformbedarfs der Regionalpolitik am Beispiel Mecklenburg-Vorpommems. 1997.

Band 7 Christian Ricken: Determinanten der Effektivität der Umweltpolitik. Der nationale Politikstil im Spannungsfeld von Ökonomie, Politik und Kultur. 1997.

Band 8 Christian Schmidt: Real Convergence in the European Union. An Empirical Analysis. 1997.

Band 9 Silvia Marengo: Exchange Rate Policy for MERCOSUR: Lessons from the European Union. 1998.

Band 10 Jens Kleinemeyer: Standardisienung zwischen Kooperation und Wettbewerb. Eine spieltheoretische Betrachtung. 1998.

Band 11 Stefan M. Golder. Migration und Arbeitsmarkt. Eine empirische Analyse der Performance von Ausländern in der Schweiz. 1999. 


\section{Hermann-Josef Berk / Hans-Günter Meissner /}

Wolf-Dieter Stelzner (Hrsg.)

\section{Gute Arbeit - Schlechte Arbeit}

\section{Sozialanalytische Zugänge zum Begriff der Arbeit}

Frankfurt/M., Berlin, Bern, New York, Paris, Wien, 1998. 157 S., 8 Abb.

O Wenn in Deutschland über "Arbeit” gesprochen und debattiert wird, kreist der Diskurs in der Regel um den Problemkomplex "Arbeitslosigkeit". Dies ist verständlich, da die Arbeitslosigkeit das sozial- und gesellschaftspolitische Thema Nr. 1 ist. Mit Hilfe sozialanalytischer Zugänge wird die Komplexität des Arbeitsbegriffes im Spannungsfeld unbewußter, gruppendynamischer, unternehmenspolitischer, kirchen- und allgemeingeschichtlicher Entwicklungen aufgefächert. Das Thema "Arbeitslosigkeit" führt zu einem Zerrbild, das der Komplexität des Phänomens "Arbeit" nicht gerecht wird. Der Tagungsband gibt Anregungen zu einem erweiterten Arbeitsbegriff.

Aus dem Inhalt: Anregungen zu einem erweiterten Arbeitsbegriff . Komplexität des Arbeitsbegriffes im Spannungsfeld unbewußter, gruppendynamischer, unternehmenspolitischer, kirchen- und allgemeingeschichtlicher Entwicklungen

Frankfurt/M - Berlin - Bern - New York - Paris - Wien

Auslieferung: Verlag Peter Lang AG

Jupiterstr. 15, CH-3000 Bern 15

Telefax (004131) 9402131

*inklusive Mehrwertsteuer

Preisänderungen vorbehalten 